\title{
Neural correlates of (dys)fluent reading acquisition in typically reading and dyslexic children
}

Citation for published version (APA):

Zaric, G. (2016). Neural correlates of (dys)fluent reading acquisition in typically reading and dyslexic children. [Doctoral Thesis, Maastricht University]. Maastricht University. https://doi.org/10.26481/dis.20160203gz

Document status and date:

Published: 01/01/2016

DOI:

10.26481/dis.20160203gz

Document Version:

Publisher's PDF, also known as Version of record

\section{Please check the document version of this publication:}

- A submitted manuscript is the version of the article upon submission and before peer-review. There can be important differences between the submitted version and the official published version of record.

People interested in the research are advised to contact the author for the final version of the publication, or visit the DOI to the publisher's website.

- The final author version and the galley proof are versions of the publication after peer review.

- The final published version features the final layout of the paper including the volume, issue and page numbers.

Link to publication

\footnotetext{
General rights rights.

- You may freely distribute the URL identifying the publication in the public portal. please follow below link for the End User Agreement:

www.umlib.nl/taverne-license

Take down policy

If you believe that this document breaches copyright please contact us at:

repository@maastrichtuniversity.nl

providing details and we will investigate your claim.
}

Copyright and moral rights for the publications made accessible in the public portal are retained by the authors and/or other copyright owners and it is a condition of accessing publications that users recognise and abide by the legal requirements associated with these

- Users may download and print one copy of any publication from the public portal for the purpose of private study or research.

- You may not further distribute the material or use it for any profit-making activity or commercial gain

If the publication is distributed under the terms of Article $25 \mathrm{fa}$ of the Dutch Copyright Act, indicated by the "Taverne" license above, 


\section{Neural correlates of (dys)fluent reading acquisition}

in typically reading and dyslexic children

Gojko Žarić 
(C) Gojko Žarić, Maastricht 2016

All rights reserved. No part of this publication may be reproduced, stored in a retrieval system or transmitted in any form or by any means, electronic, mechanical, photocopying, recording or otherwise, without prior written permission of the publisher.

The work in this thesis was funded by the Netherlands Organization for Scientific Research (NWO) through National Initiative Brain and Cognition (NIHC) as a part of the research program "Fluent reading acquisition neurocognitively decomposed: the case of dyslexia (HCMI 10-59)" under grant number 056-14-015.

Cover: Gorka Fraga González \& Gojko Žarić

Production: CPI Wöhrmann Print Services, B.V.

ISBN 978-94-6203-977-3 


\section{Neural correlates of (dys)fluent reading acquisition in typically reading and dyslexic children}

\section{DISSERTATION}

to obtain the degree of Doctor at Maastricht University, on the authority of the Rector Magnificus, Prof. Dr. L.L.G. Soete in accordance with the decision of the Board of Deans, to be defended in public on Wednesday $3^{\text {rd }}$ February 2016 at 12:00 hours by

Gojko Žarić 


\section{Supervisors:}

Prof. Dr. Bernadette M. Jansma

Prof. Dr. Maurits W. van der Molen (University of Amsterdam, NL)

\section{Co-supervisors:}

Dr. Milene L. Bonte

Dr. Jurgen Tijms (IWAL Institute, Amsterdam, NL)

\section{Assessment Committee:}

Prof. Dr. Sonja A. Kotz (Chair)

Prof. Dr. Paavo H.T. Leppänen (University of Jyväskylä , Finland)

Dr. Nienke M. van Atteveldt (VU University Amsterdam, NL)

Dr. Lisa M. Jonkman 


\section{Contents}

1 General Introduction $\quad 7$

2 Reduced neural integration of letters and speech sounds in dyslexic children scales with individual differences in reading fluency

3 Crossmodal deficit in dyslexic children: practice affects the neural timing of letter-speech sound integration

$4 \quad$ Altered patterns of effective connectivity within the reading network of dyslexic children and their relation to reading dysfluency

$5 \quad$ Altered white matter connectivity in 8-10 year old dyslexic and typically reading children

6 Summary

$7 \quad$ Knowledge valorization

Acknowledgements

Publications

Curriculum Vitae $\mathrm{BC}$ 



\section{CHAPTER 1}

General introduction 
Reading is a complex cognitive function that enables us to rapidly extract meaning from arbitrary visual shapes that connect to speech sounds. Reading and its complement, writing, together compose literacy (UNESCO, 2006). This ability, though seemingly uncontroversial, is of such great political importance that there is still debate on the social aspects that it holds and how it should be taught, e.g. leading to "the reading wars" in the United States during the last decade of the $20^{\text {th }}$ century, in which "whole-language" and "phonics" camps competed for passing legislations regarding teaching practices, promoting intervention programs and defining criteria of acceptable research on literacy (Collins \& Blot, 2003). Literacy is now understood as a social phenomenon, rather than an individual one (UNESCO, 2005). It was set as a core goal of the UNESCO's worldwide campaign to bring "Education to All", with an accent on "the indispensable role that education - with literacy at its core - plays in bettering the lives of individuals, their communities and nations" (UNESCO, 2005). Thus, individuals incapable to achieve literacy levels determined by demands of modern society can suffer from adverse academic, economic, and psychosocial consequences (Undheim \& Sund, 2008; Undheim, Wichstrøm, \& Sund, 2011).

Developmental dyslexia is defined as "a specific learning disability that is neurobiological in origin" (Lyon, Shaywitz, \& Shaywitz, 2003). It affects 5\% to $10 \%$ of children who never acquire proficient reading skills despite normal cognitive abilities and schooling opportunities (Blomert, 2005; Lyon et al., 2003; Snowling, 2013). A lack of reading fluency has been pinned down as its most persistent and impaired characteristic (Shaywitz, Morris, \& Shaywitz, 2008), although there are inter-individual differences in the level of reading (dys)fluency among dyslexic readers (Katzir, Kim, Wolf, Morris, \& Lovett, 2008; Leinonen et al., 2001).

This thesis investigates brain networks involved in reading fluency development in dyslexia. As dyslexia is a developmental disorder, participants in our studies were 8-9 year old typical and dyslexic Dutch speaking children. We examine neurophysiological correlates of letter-speech sound coupling and visual word 
recognition in dyslexic and typical readers, but also look at differences within the group of dyslexic children based on the severity of reading dysfunction. Furthermore, we inspect the malleability of atypical responses in dyslexic readers following a reading training. In the first two studies, we investigate electroencephalographic (EEG) brain responses to simultaneously presented congruent and incongruent letters and speech sounds using an oddball paradigm. The third study examines differences in functional effective connectivity within the reading network as assessed by EEG signals to visually presented words. Finally, in the fourth study we look at the anatomical connectivity within the dyslexic and typical reading network using diffusion tensor imaging.

In this introduction we give a short overview of the steps involved in reading acquisition and underlying brain networks that subserve reading as a complex cognitive function. Furthermore, we briefly present current findings on unsuccessful reading in dyslexia and its neurophysiological sources. This is accompanied by a short discussion of the current remediation programs in dyslexia. Finally, we link the work presented in the following chapters of this thesis to the other behavioral and neuroimaging findings on (un)successful letter-speech sound integration and visual word recognition.

\section{1. (Un)successful reading acquisition}

Written language consists of letters or graphemes, i.e. characters of arbitrary forms that are by social agreement related to specific speech sounds or phonemes, i.e. perceptually distinct units of a spoken language system that can differentiate between the words of that language (e.g. $\boldsymbol{b} a \mathrm{t}$, cat, $\boldsymbol{h}$ at). Scripts in which each grapheme represents one speech sound (Latin, Greek, Cyrillic, Hangul, Armenian and Georgian) are called alphabetic (Figure 1-1). A more loose definition of alphabetic scripts includes script such as abjad in which commonly only the consonants are written (Arabic and Hebrew), syllabic (Japanese Katakana script), and abugida scripts in which the vowel does not have its own symbol but is represented by changing the letter symbol of the consonant (e.g. Indic, Ethiopic, Canadian Aboriginal). Languages using 
alphabetic scripts can vary in the consistency of the connection between grapheme and phoneme. Transparent or shallow orthographies are the ones in which there is a high regularity in the letter-speech sound connection (Zoccolotti et al., 2005), meaning that a certain letter is (almost) always connected with the same speech sound. On the other hand, opaque or deep orthographies are the ones with irregular letter-speech sound correspondences (one letter is connected to several speech sounds; Box 1-1) and the correct connection depends not only on the phonic rules, but also on the position of the letter in a word (Johnston, 2001).

In all of these scripts the first step in reading acquisition is a matching of distinctive visual symbols (graphemes) to units of speech sound (phonemes), also known as phonological recoding (Ziegler \& Goswami, 2005) ${ }^{1}$. This means that, rather than mapping orthography directly to existing phonological representations of speech sounds, there is an ongoing recoding of the relevant spoken language elements, as it is unlikely that the speech sounds have isolated representations before learning to read (Blomert \& Froyen, 2010; Jose Morais, Alegria, \& Content, 1987; Wimmer, Landerl, Linortner, \& Hummer, 1991). For example, illiterate adults and literate adults reading exclusively logographic scripts are worse on phonological tasks than the literate adults reading alphabetic scripts (Hohn \& Ehri, 1983; José Morais, Cary, Alegria, \& Bertelson, 1979; José Morais, Castro, Scliar-Cabral, Kolinsky, \& Content, 1987; Read, Zhang, Nie, \& Ding, 1986). Furthermore, before they learn the alphabet, prereading children memorize easier spellings with distinctive visual properties, though they have no connections to speech sounds, for example YMPL for chicken (Ehri \& Wilce, 1985). When the children obtain alphabetic knowledge, there is a shift from visual to phonological cueing, and the recognition of phonologically cued spellings, e.g. HKN for chicken, becomes superior to salient visual spellings (Ehri \& Wilce, 1985; Roberts, 2003). Both, knowledge of grapheme-phoneme relations and the ability to distinguish separate phonemes, so called phonemic awareness, are needed for the next step, named sight word

\footnotetext{
${ }^{1}$ Logographic scripts, such as Chinese, in which graphemes already represent morphemes, are out of the scope of this thesis.
} 
reading in which familiar words are read rapidly as units without any need to consciously process letter-speech sound correspondences (Ehri, 2005).

Box 1-1. "Gee each owe tea eye smells fish." James Joyce, Finnegan's Wake

An example of an opaque orthography is English in which same letter may represent different phonemes. To illustrate these irregularities the nonword "ghoti" was constructed (attributed to George Bernard Shaw). If the sounds are read as follows: the "gh" as /f/ like in rough, the "o" as /e/ like in women, and the "ti" as /sh/ like in nation, the pronunciation of "ghoti" would be the same as for "fish" (Johnston, 2001). Moreover, "ghoti" could be completely made out of silent letters, and consequently silent itself, if we would use: "gh" as in light, "o" as in people, " $t$ " as in ballet, and " $\mathrm{i}$ " as in business. Of course, these pronunciations would only be correct if the English letter-speech sound correspondences were regular and unambiguous, but as English has a deep orthography, these correspondences are defined also by the position in a word and not just by phonics rules (Johnston, 2001).

There are a several lines of evidence showing that experienced readers perceive visually presented word differently than merely processing its parts separately in succession. First, it seems that the time needed to recognize a word is not always influenced by its length (Nazir, Jacobs, \& O'Regan, 1998; New, Ferrand, Pallier, \& Brysbaert, 2006). Second, a letter is recognized faster if it is embedded in a word than in isolation or a random letter string, the so called word superiority effect (Reicher, 1969). Third, the superiority effect extends to pseudowords, i.e. meaningless letter strings which are constructed by the structural rules of the writing system (Baron \& Thurston, 1973) implicating that the effect is prelexical and easily generalizable to new stimuli (McCandliss, Cohen, \& Dehaene, 2003). Fourth, recognition is almost perfect even when the word is presented only for a very brief amount of time, such as $50 \mathrm{~ms}$ (Rayner, Pollatsek, \& Schotter, 2012). Finally, these effects are invariant to variations in 
size, case, and font, and are influenced by priming across such variations (McCandliss et al., 2003). On the other hand, this does not mean that there is a stage in development where there is a switch to exclusively sight word reading, but rather a shift from primarily relying on phonological recoding strategies to predominantly sight word reading (Vaessen \& Blomert, 2010).

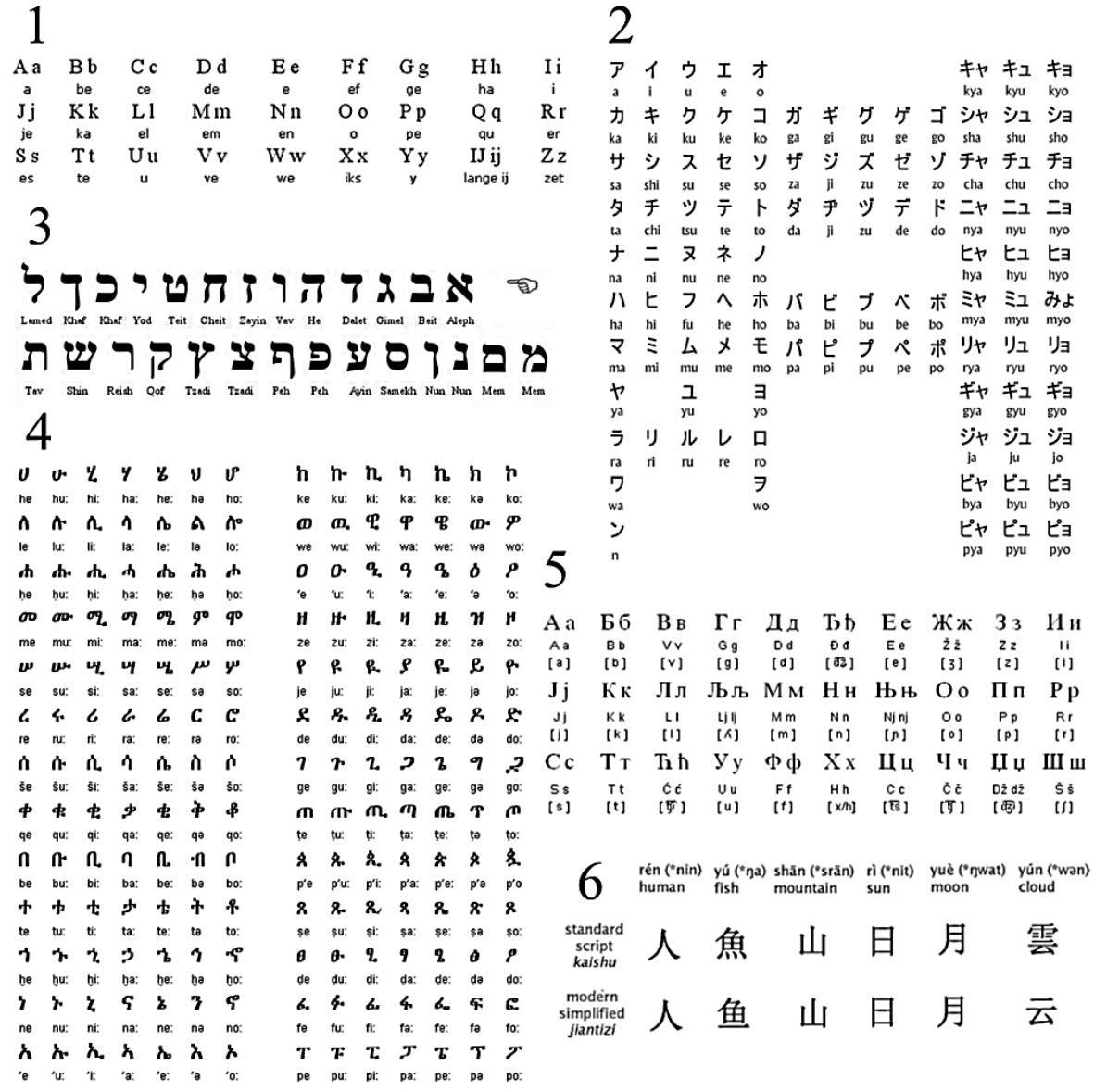

Figure 1-1. Examples of different scripts: 1) alphabet - Dutch 2) syllabic - Japanese Katakana 3) abjad - Hebrew 4) abugida - Ethiopic 5) alphabet - Serbian Cyrillic and Latin 6) logographic - Chinese standard and modern simplified (examples)

Successful acquisition of fluent word reading is dependent on multiple factors. For example, a large scale study in three languages with differing level of 
transparency between letters and speech sounds (Dutch, Portuguese, Hungarian) tested 6 factors (Vaessen et al., 2010): speed of naming visually presented letters, digits, or objects (Rapid Naming factor), accuracy and speed of choosing the letter that corresponds to an auditorily presented phoneme, and of same/different judgments of simultaneously presented letters and phonemes (Letter-Speech Sound Accuracy and Speed factors), accuracy of repetition of an auditorily presented sequences of consonants and syllables (Verbal Working Memory factor), and the speed and accuracy of phoneme omission from auditorily presented pseudowords (Phonological Awareness Accuracy and Speed factors). Vaessen et al. (2010) showed a large contribution of letter-speech sound accuracy, phonological awareness accuracy and speed, and rapid naming factors to reading fluency. Interestingly, letter-speech sound and phonological awareness factors were larger contributors at the beginning of primary school (first grade) and their influence declined over time. Conversely, the contribution of the rapid naming factor increased with reading experience (Vaessen et al., 2010). This is in line with the hypothesized steps of reading acquisition mentioned in the previous paragraph, with letter-speech sound and phonological awareness being needed to gradually shift to sight word reading. Dyslexic readers typically show impaired performance on tasks related to each of the factors that mostly contribute to reading fluency. Behavioral impairments were found for example in phonological awareness and rapid automatized naming, which also show similar developmental differences with phonological awareness being impaired in early grades and rapid naming in later school years (de Jong \& van der Leij, 2003), and letter-speech sound coupling at the beginning of reading instruction in children at familial risk for dyslexia (Blomert \& Willems, 2010). Furthermore, the reading impairment in dyslexic readers is particularly detectable on unfamiliar words and pseudoword reading tasks for which letter by letter reading is required, rather than for familiar words (Bruck, 1990; S. E. Shaywitz \& Shaywitz, 2005; Wimmer, 1996).

Reading acquisition involves a concurrent development of phonological awareness and letter-speech sound associations. While in typically reading children phonological awareness improves when they learn to read (Mann \& 
Wimmer, 2002; Wimmer et al., 1991), dyslexic children show letter-speech sound integration and phonological awareness impairments (Blomert \& Willems, 2010; de Jong \& van der Leij, 2003). Moreover, further supporting importance of these factors for successful typical reading development, these impairements were related to reading dysfluency more at the beginning of the reading instruction than in the later school years. Hence, two mutually nonexclusive accounts were proposed as an underlying source of dyslexia.

The most prevalent view on the underlying source of dyslexia is the phonological deficit theory. Dyslexic readers usually exhibit impaired phonological awareness and limited verbal working memory (Snowling, 1998), though it is still a matter of debate whether dyslexic readers cannot build clear phonological representations or they cannot efficiently use otherwise intact phonological representations (Ramus \& Szenkovits, 2008). Though it is the most accepted theory, there are difficulties in explaining all the results with a purely phonological account. For example, sometimes a letter-speech sound deficit is present without obvious impairments in phonological awareness (Blomert \& Willems, 2010). Furthermore, interpreting the results of phonological tasks as purely phonological is not as straightforward as it seems. Thus, studies have shown that orthographic influences can be seen in verbal phonological tasks (Cone, Burman, Bitan, Bolger, \& Booth, 2008; Dijkstra, Roelofs, \& Fieuws, 1995; Seidenberg \& Tanenhaus, 1979; Ziegler \& Ferrand, 1998), meaning that even without presenting written stimuli, orthographic knowledge influences phonological judgements (Bishop, 2006). Based on these findings as well as neuroimaging evidence discussed below, a letter-speech sound integration deficit has been proposed as a more proximal cause of reading deficits in dyslexia in transparent alphabetical scripts (Blomert, 2011; Ehri, 2005; Share, 1995; Wimmer \& Schurz, 2010). This proposition does not exclude the possibility of phonological deficit in dyslexic readers, but sees it as a more distal cause of reading failure (Blomert \& Willems, 2010) which influence may be greater in opaque than in transparent languages (Mann \& Wimmer, 2002). 


\subsection{Reading deficit as a multi-sensory problem}

Literacy was invented less than 5000 years ago and as such it is probably phylogenetically too recent to have a priori predisposed brain areas. Instead it is subserved by two evolutionary developed neural systems, predominantly located in the left hemisphere (Schlaggar \& McCandliss, 2007), one for spoken language (Liberman, Shankweiler, Fischer, \& Carter, 1974; Pugh, Mencl, Jenner, Lee, et al., 2001), and the other for visual object recognition (Dehaene, Cohen, Sigman, \& Vinckier, 2005). As these systems are not originally devised for reading, they have to adapt to the newly obtained reading skill (Brem et al., 2010; Castro-Caldas, Petersson, Reis, Stone-Elander, \& Ingvar, 1998; Dehaene et al., 2010). Thus, we can look at reading acquisition as a multisensory problem in which a novice reader has to combine a visual unit (a letter) with an auditory unit (a speech sound) into a new audiovisual unit, while also learning to extract speech sound from the otherwise continuous auditory input to make a new representation of a speech sound including its orthographic characteristics, so called graphonemes (Whitney \& Cornelissen, 2005).

Studies using letter-speech sound correspondences to investigate their integration in typical and dyslexic readers have shown reduced integration in dyslexics both in terms of behavioral (Aravena, Snellings, Tijms, \& van der Molen, 2013; Blomert \& Willems, 2010; Snowling, 1980) and neural measures (Blau et al., 2010; Blau, van Atteveldt, Ekkebus, Goebel, \& Blomert, 2009; Froyen, Willems, \& Blomert, 2011; Žarić et al., 2014). On the other hand, there is also evidence showing that dyslexic readers are impaired on non-linguistic audiovisual tasks (Harrar et al., 2014), as well as that their audiovisual speech perception is more effortful if not impaired (Pekkola et al., 2006; but see: Baart, De Boer-Schellekens, \& Vroomen, 2012; Groen \& Jesse, 2013).

The difference between letter-speech sound and natural audiovisual integration is twofold. First, though both visual and auditory systems are involved in letterspeech sound coupling, there is an asymmetry in their interaction as the audiovisual information is usually not simultaneous, but phonological information is internally generated based on letters. Moreover, in certain 
experimental paradigms, it seems that while letter presentation influences speech sound processing (Froyen, Van Atteveldt, Bonte, \& Blomert, 2008), the reverse does not seem to be the case (Froyen, van Atteveldt, \& Blomert, 2010). This asymmetry is not seen in natural audiovisual integration, such as audiovisual speech, and it may stem from the fact that letter-speech sound pairs are in principle arbitrary associations, dependent on a specific orthography (Blomert \& Froyen, 2010). A second specificity of letter-speech sound associations is their larger dependence on temporal proximity than in natural audio-visual integration, i.e. if the letter and speech sound are not presented synchronously or in close temporal succession, they will not be perceived as a perceptual unit (Blomert \& Froyen, 2010). Furthermore, letter-speech sound pairs are highly overlearned and may differ in familiarity from other artificial audiovisual objects used in experiments and as such they may also be processed in a different way (Blomert \& Froyen, 2010; Hein et al., 2007; Naumer et al., 2009). For example, studies have shown that unfamiliar artificial audiovisual stimuli activate primarily inferior frontal cortex, while familiar audiovisual stimuli activate superior temporal sulcus (Naumer et al., 2009), an area also involved in letter-speech sound integration (van Atteveldt, Formisano, Goebel, \& Blomert, 2004). As a result, although there are studies showing that dyslexics perform worse on linguistic and non-linguistic audiovisual materials it is still not possible to conclude whether impaired letter-speech sound integration is a specific deficit, or whether it stems from a more fundamental impairment in audiovisual multisensory integration (Hahn, Foxe, \& Molholm, 2014).

\subsection{Reading training}

As the phonological deficit is seen by many as the proximal cause of dyslexia, most reading interventions and trainings focus on training phonemic awareness through training children to accurately associate letters to speech sounds. Accordingly, while reading interventions can help to achieve reasonable levels of reading accuracy, i.e. the ratio of the correctly read words and words that participants read (Alexander \& Slinger-Constant, 2004; Hatcher et al., 2006; Lovett, Barron, \& Benson, 2003; Tijms, 2007), reading fluency (the number of correctly read words in a limited amount of time, usually 60 seconds) hardly 
improves with most of the current trainings (Alexander \& Slinger-Constant, 2004; Compton, Miller, Elleman, \& Steacy, 2014; Gabrieli, 2009; Hintikka, Landerl, Aro, \& Lyytinen, 2008; Landerl \& Wimmer, 2008; Thaler, Ebner, Wimmer, \& Landerl, 2004; Torgesen et al., 2001). A small number of studies that report on improved fluency stress a need for multi-component interventions which also include modules for fluency and text comprehension (Levy, Abello, \& Lysynchuk, 1997; Wolff, 2011), though it is not clear from these studies whether the gains were generalized to untrained materials. Improvement in reading fluency beyond accuracy is of great importance as in fairly transparent orthographies, for example Dutch or German, the fluency impairment is most perceivable characteristic of dyslexia (Blomert, 2011; Wimmer, 1993, 1996) and it has been stressed as an important and most vicious trait of dyslexia (Blomert, 2011; Katzir et al., 2006; S. E. Shaywitz \& Shaywitz, 2008).

Furthermore, even if the dyslexic readers as a group improve after training, it does not mean that the training was successful for all of them, i.e. some participants are 'nonresponders' (Al Otaiba \& Fuchs, 2002). Some estimations are that between $2 \%$ and $6 \%$ of the children in $1^{\text {st }}$ and $2^{\text {nd }}$ grade will be unresponsive to reading intervention and will remain poor readers (Torgesen, 2000). Although phonological awareness, rapid naming and word identification fluency were identified as predictors of the response to an early intervention, as well as socio-economic status (Al Otaiba \& Fuchs, 2002; Dukleth Johnson \& Lee Swanson, 2011; McMaster, Fuchs, Fuchs, \& Compton, 2005; Nelson, Benner, \& Gonzalez, 2003; Toste et al., 2014; Tran, Sanchez, Arellano, \& Lee Swanson, 2011), this list is far from complete (Scheltinga, van der Leij, \& Struiksma, 2010).

The work in this thesis and the complementary thesis of Gorka Fraga González (University of Amsterdam) aims to investigate different factors contributing to the fluency barrier in dyslexic children. Crucially, we investigated a training program inspired by the multisensory integration deficit account of dyslexia to test whether and how the training to integrate letters and speech sounds can 
improve reading fluency. The training was a modified version of a computerassisted intervention program implemented by the IWAL institute (Tijms, 2004, 2007, 2011), focusing not only on letter-speech sound integration accuracy, but also on its automation. First, letter-speech sound correspondences were explicitly trained until each child achieved a mastery level. Second, children were provided with a high exposure to the trained letter speech sound associations to stimulate the automatic integration of letters and speech sounds. A group of dyslexic children following the training for 6 months was compared to dyslexic and typical children without the training. At the behavioral level, we found that dyslexic children following the treatment improved more in fluency than both other groups, although they did not reach the level of typical readers (Fraga González et al., 2015). Chapter 3 of this thesis presents an in-depth investigation of the plasticity of neural letter-speech sound integration by means of intervention expressed in electrophysiological parameters.

\section{Neuroimaging of the typical and dyslexic reading brain}

Neuroimaging methods, that allow us to noninvasively investigate activation of brain in vivo while participants are performing different cognitive tasks, have shed a light on the cortical reading network. Two main methods in neuroscientific research on reading are functional magnetic resonance imaging (fMRI) and electroencephalography (EEG).

Functional MRI is used to identify brain regions involved in reading related cognitive tasks, such as letter-speech sound integration and visual word recognition, by means of metabolic demands coupled with neuronal activity. It profits from magnetic properties of oxygenated and deoxygenated hemoglobin in blood (Ogawa, Lee, Kay, \& Tank, 1990), as more oxygen is transported to the areas that are more active during the task of interest relative to a control state (rest or other task). This method has a high spatial resolution, but a limited temporal resolution of several seconds, due to sluggish hemodynamic characteristics. Conversely, EEG has a high temporal resolution of a few milliseconds, but a low spatial resolution of a few centimeters, due to volume conduction. It is a measure of the cortical electrical activity at the surface of the 
head. A synchronous firing of large numbers of mainly parallel pyramidal neurons in the cortex produces a sufficiently large electric field that can be measured on the external surface of the scalp using EEG (Luck, 2005).

Neuroimaging studies have delineated the networks of brain regions involved in letter - speech sound integration and visual word recognition. For instance, a ventral left occipito-temporal region, and specifically left fusiform gyrus, is involved in fast visual word recognition (Jobard, Crivello, \& Tzourio-Mazoyer, 2003; McCandliss et al., 2003), while regions in the superior temporal cortex are involved in multisensory grapheme-phoneme integration (Blomert, 2011; Simos et al., 2002; van Atteveldt \& Ansari, 2014). Furthermore, learning to read enhances brain activation in a widespread network (Brem et al., 2010; Dehaene et al., 2010) including regions involved in phonological processing (Brennan, Cao, Pedroarena-Leal, McNorgan, \& Booth, 2013; Monzalvo \& DehaeneLambertz, 2013; van Atteveldt \& Ansari, 2014).

Most neuroimaging studies have focused on adult participants, but a need to investigate reading acquisition and the plasticity of the developing brain to get a whole picture stresses the importance of studies with children of different ages. For example, a recent meta-analysis of fMRI studies shows that both adults and children use the same reading network, but have different peaks of activations, with adults showing more activity in posterior occipito-temporal cortex and children in left superior temporal and bilateral supplementary motor regions (Martin, Schurz, Kronbichler, \& Richlan, 2015). This would be in line with the proposed reading development from letter-speech sound integration (left superior temporal regions) to sight word reading (occipito-temporal cortex).

Further differences between children and adults come from EEG studies, and especially from the mismatch negativity paradigm (MMN). In this paradigm at a latency of 100-200 ms, a mismatch negativity (MMN) response is elicited by a rare sound (deviant, or oddball) that is presented in a sequence of frequent (standard) sounds (Näätänen, 2001). It is believed that the MMN represents an automatic change detection response sensitive to deviation from traces in 
auditory short-term memory (Näätänen, Paavilainen, Rinne, \& Alho, 2007; Näätänen, 2001). Additionally, in school-aged children the speech evoked MMN response may be followed by late negativity (Late MMN or LN) in a broader time window from 300 to $700 \mathrm{~ms}$, while it decreases in adulthood (Cheour, Korpilahti, Martynova, \& Lang, 2001; Czamara et al., 2011; Hommet et al., 2009). This could suggest that the developing brain needs additional processing resources compared to adult brain to reach to the same result (see also Chapter 2). Furthermore, while studies investigating detection of the subtle speech changes involving stop consonants found differences in speech evoked MMN and LN responses (Csépe, 2003; Schulte-Körne et al., 1998), the studies using single vowels found no impairments in the speech evoked responses of the dyslexics (Froyen et al., 2011; Žarić et al., 2014, 2015). On the other hand, these studies found differences between typical land dyslexic readers in letterspeech sound integration.

A series of crossmodal MMN studies, investigating letter-speech sound integration, found that adults showed an early enhancement of the speech evoked MMN due to the simultaneous presentation of letters (Froyen et al., 2008), while 7 to 11 year old children additionally showed later LN letter effects and a developmental shift in the temporal integration window (Froyen, Bonte, van Atteveldt, \& Blomert, 2009; Žarić et al., 2014). This finding was surprising as it showed that, although in fairly transparent language like Dutch accurate letter-speech sound identification and discrimination are typically observed after one year of reading instruction (Blomert \& Vaessen, 2009), it takes years for the development of the automatic neural integration of these associations (Froyen et al., 2009, 2008; Žarić et al., 2014). Likewise, EEG studies investigating visual word recognition showed a slow developmental tuning to orthographic stimuli of a negative evoked potential around $200 \mathrm{~ms}$, usually labeled N1 or N170 (Maurer, Brem, Bucher, \& Brandeis, 2005). Interestingly, crossmodal MMN, N1 word-symbol differences and phonological priming effects in typically reading children each suggest an inverted " $U$ " model of development with larger condition effects for $2^{\text {nd }}$ and $3^{\text {rd }}$ grade children relative to both younger and older children and adults (Bonte \& Blomert, 2004; Froyen 
et al., 2009, 2008; Maurer et al., 2006, 2011; Žarić et al., 2014). These patterns of results advocate for a slow/protracted development and a high sensitivity to reading related stimuli (phonemes, letter-speech sound pairs, words) in the first years of reading acquisition.

Similar to the electrophysiological studies, fMRI studies also found differences between typical and dyslexic readers on phonological, orthographicphonological integration or visual word recognition tasks. Given the scope of the thesis, we focus here on the letter-speech sound integration and visual word recognition. The studies investigating multimodal integration reported underactivation of the left posterior superior temporal sulcus/gyrus (Blau et al., 2010, 2009; McNorgan, Randazzo-Wagner, \& Booth, 2013; B. A. Shaywitz et al., 2002). Conversely, studies investigating visual word recognition have found hypoactivation in the left occipito-temporal regions (Monzalvo, Fluss, Billard, Dehaene, \& Dehaene-Lambertz, 2012; B. A. Shaywitz et al., 2007; van der Mark et al., 2009). These regions were also consistently found as hypoactivated in dyslexic participants in meta-analyses (Maisog, Einbinder, Flowers, Turkeltaub, \& Eden, 2008; Richlan, Kronbichler, \& Wimmer, 2009). In some cases, presumably as a compensatory mechanism, a hyperactivation of prefrontal dorsal sites and right-hemisphere homologues were found (Pugh, Mencl, Jenner, Katz, et al., 2001; B. A. Shaywitz et al., 2002).

While fMRI studies point to place(s) of altered brain activation in dyslexic as compared to typical readers, EEG studies give us an insight in the timing of the disruptions during the task. Studies using the crossmodal MMN paradigm point to typical vowel-evoked auditory change detection responses and reduced crossmodal effects in dyslexic children (Froyen et al., 2011; Žarić et al., 2014). In particular, in chapter 2 of this thesis, we look at individual differences within the dyslexic group (Žarić et al., 2014). We show reduced letter-speech sound integration in 9 year old dyslexic participants, with different patterns of integration deficiency depending on the level of reading dysfluency. Moreover, individual differences in reading fluency were correlated with the latency of the MMN response to simultaneously presented letters and speech sounds (Ž́arić et 
al., 2014). These results stress the importance of investigating within group individual differences as it could be of interest for the prediction of remediation outcomes and individual tailoring of the type of reading remediation. Hence, the next question was whether training can change these responses? In Chapter 3, we investigated whether 9-year old dyslexic children exhibit changes in crossmodal MMN and LN responses following systematic letter-speech sound integration training and whether these changes relate to behavioral improvements in reading fluency and letter-speech sound coupling (Žarić et al., 2015). Our findings suggest moderate improvements of the reduced neural integration of letters and speech sounds with reading instruction and training, particularly in the timing of later aspects of this integration (LN window). The early integration (MMN window) seems to be more treatment resistant, with individual differences in its timing prior to training predicting gains in reading fluency.

To study the network of brain regions involved in word reading, we measured electrophysiological responses in typical and dyslexic readers during a visual word recognition task. We presented words vs. strings of meaningless letter-like symbols to examine the specificity of the N1 component in 9 year old dyslexic participants (Fraga González et al., 2014). Both typical and dyslexic readers showed a more pronounced N1 component for words compared to symbols. Interestingly, the N1 responses to words were larger over left hemisphere in the dyslexic group than in typical readers suggesting facilitated lexical access or more efficient attention allocation in the group of typically reading children. Moreover, N1 amplitudes and reading fluency were positively related in the dyslexic group. Furthermore, we investigated the changes of the N1 component for words vis-à-vis responsiveness to training (Fraga González et al., submitted). Responders showed a significant reduction of visual N1 amplitudes for words relative to the pretest, while this effect was absent in poor responders. Interestingly, we found a moderately positive relation between gains in reading fluency and the training-related reduction in $\mathrm{N} 1$ responses. In the present thesis we further analyzed the EEG responses to the visual words and symbols to investigate functional effective connectivity within the reading 
network of typical and dyslexic children prior to training (see below and Chapter 4). In sum, our results, from both letter-speech sound integration and visual word recognition, with and without training, stress the importance of (1) measuring brain activity reflecting reading-related skills and (2) behavioral measures of reading fluency and not just accuracy, as well as (3) considering individual differences in reading fluency rather than just focusing on group comparisons.

\section{Reading networks}

Reading is a complex cognitive function and it is likely that it depends on connectivity within large scale neuronal networks, rather than on strictly localized processes (Engel, Fries, \& Singer, 2001). The neural reading network can be divided in two parts (Figure 1-2): (1) an anterior part that includes left inferior-frontal regions related to speech production and word phonological recoding (Sandak, Mencl, Frost, \& Pugh, 2004), and (2) a posterior part which includes left posterior temporal and dorsal parieto-temporal areas involved in phonological processing (Pugh, Mencl, Jenner, Katz, et al., 2001) and multisensory grapheme-phoneme integration (Blomert, 2011; Simos et al., 2002; van Atteveldt \& Ansari, 2014) and left ventral occipito-temporal region involved in fast visual word recognition (Jobard et al., 2003; McCandliss et al., 2003).

There are two main ways to investigate connections within the reading network. We can look at functional or anatomical connectivity between the different parts of the network. The first tells us either about the influence of one brain region on other regions, so called effective connectivity, or about the extent to which activation in different brain regions is correlated, so called functional connectivity (Cao, Bitan, \& Booth, 2008), while the latter estimates the white matter connections between brain regions and the properties of these connections (Vandermosten, Boets, Wouters, \& Ghesquière, 2012). These methods give us the opportunity to study disruptions in connectivity between areas, rather than a dysfunction of a single area as a possible cause of dyslexia. 


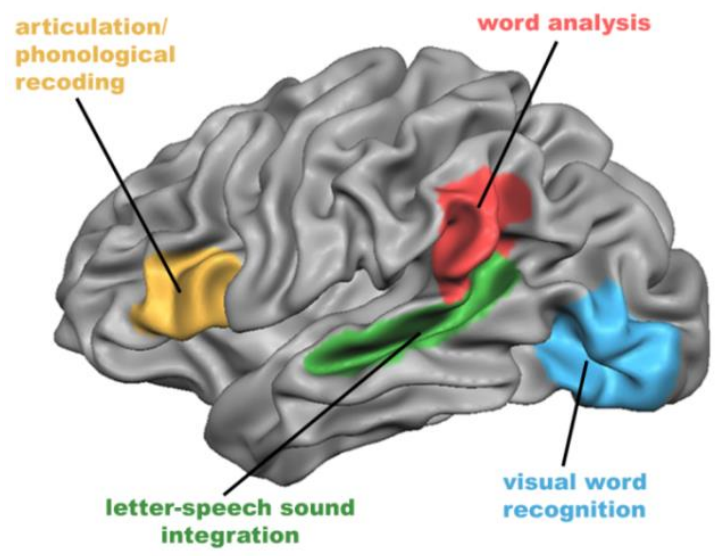

Figure 1-2. The left lateralized reading network

\subsection{Functional connectivity}

There are multiple ways to calculate EEG based functional or effective connectivity. First, we can look at coherence which can be described as correlation in the frequency domain between two channels (Varela, Lachaux, Rodriguez, \& Martinerie, 2001). Unfortunately, coherence cannot be so easily interpreted as volume conduction and the influence of common reference electrodes can cause large coherence values even when there is no real cortical interaction (Schlögl \& Supp, 2006). Several other approaches are proposed to overcome these problems. Directed transfer function (DTF), an analog of partial coherence, is the ratio between the input form one channel to another and all the other inputs to that channel (Blinowska, Kuś, \& Kamiński, 2004). Partial-directed coherence (PDC), on the other hand, is the ratio between the output from one channel to another and all the other outputs from that channel (Baccalá \& Sameshima, 2001). Finally, another approach is to use imaginary part of coherence (iCOH) as it is hypothesized that it is only sensitive to synchronizations of two processes which are time-lagged to each other and not to volume conduction which does not cause a time-lag (Nolte et al., 2004).

EEG studies investigating functional connectivity yielded a mixed pattern of results. While studies using tasks found reduced or more diffuse EEG 
coherence in poor relative to typical readers on letter identification and acoustic tasks (Dhar, Been, Minderaa, \& Althaus, 2010; Nagarajan et al., 1999) others measuring coherence of the resting state found the opposite (Arns, Peters, Breteler, \& Verhoeven, 2007; Shiota, Koeda, \& Takeshita, 2000) or increased coherence for some EEG bands and reduced coherence for other bands (Marosi et al., 1995). Fraga González et al. (submitted) examined differences in the topological properties of brain resting-state networks between 9 year old dyslexics and typically reading children using graph analysis. In agreement with the results from the studies employing tasks, the results of this study suggest a less integrated network and less communication between the nodes in dyslexia. Moreover, fMRI studies found disrupted connectivity between anterior and posterior parts of the reading network in dyslexic adults (Schurz et al., 2014; S. E. Shaywitz et al., 2003; Stanberry et al., 2006) and children (Cao et al., 2008; Quaglino et al., 2008; van der Mark et al., 2011). Concordant with hyperactivation of the anterior part of the network in dyslexia, a stronger connectivity between the frontal regions of the left and right hemisphere was reported for dyslexic children (Richards \& Berninger, 2008).

In chapter 4, we use DTF to investigate effective connectivity during visual word recognition in the same groups of children in which we found differences in crossmodal letter-speech sound integration dependent on their level of reading (dys)fluency (Žarić et al., 2014). We aimed to investigate whether the level of reading dysfluency also affected effective brain connectivity during visual word processing. Our findings suggest stronger posterior to anterior connectivity within occipito-temporal sites in typical readers than in both dyslexics groups. Conversely, dyslexic groups exhibited stronger anterior to posterior connectivity and employment of sites over right hemisphere that scaled with reading dysfluency. These differences between the two dyslexic groups could explain at least in part the mixed results of the previous EEG/MEG functional connectivity studies. 


\subsection{Anatomical connectivity}

Today MRI scanners are also routinely used for diffusion weighed imaging (DWI) as a standard method for noninvasive imaging of the white matter tracts. It profits from the properties of a water diffusion process in the brain. The neural tissue of the brain constrains diffusion of water molecules. Water is constrained, for example, by cell membranes, cytoskeleton, and macromolecules (Johansen-Berg \& Behrens, 2009). Cells hinder the motion of both extracellular and intracellular water molecules. As grey matter does not have an oriented fiber structure water is diffusing in different directions, i.e. it has largely isotropic diffusivity. On the other hand, white matter consists of myelinated axons running parallel to each other, and the diffusion will be larger along the axons than perpendicular to them, as it is constrained by the myelination, axonal membranes and the neurofibrils (Beaulieu, 2002). This diffusion along a preferred direction is called anisotropic diffusion. The usually reported index of anisotropy is fractional anisotropy (FA), which is 0 for isotropic diffusion and it becomes closer to 1 the larger the anisotropy is.

Evidence from studies comparing white matter between adult typical and impaired readers commonly found lower FA values in parieto-temporal regions, including the arcuate fasciculus and other parts of the superior longitudinal fasciculus (Frye et al., 2011; Richards et al., 2008; Steinbrink et al., 2008; Thiebaut De Schotten, Cohen, Amemiya, Braga, \& Dehaene, 2014; Vandermosten, Poelmans, Sunaert, Ghesquière, \& Wouters, 2013; Vandermosten, Boets, Poelmans, et al., 2012) that connect the frontal and parieto-temporal regions, as well as in the anterior and posterior limb of the left internal capsule and the corona radiata (Klingberg et al., 2000; Richards et al., 2008) connecting the thalamus to the cerebral cortex and the fronto-parietal cortex to subcortical nuclei and spinal cord. There is still debate whether the corona radiata is involved in reading or the method used was not sensitive enough and the cluster with significant differences belonged to a different horizontally running tract (Vandermosten, Boets, Wouters, et al., 2012). Furthermore, typical and dyslexic readers differed in white matter properties of corpus callosum, including body, splenium and isthmus (Frye et al., 2008; 
Richards et al., 2008; Welcome \& Joanisse, 2014) where the latter two connect the occipital and temporal lobes. Finally, group differences were also found in long association fibers connecting the ventral occipital to the anterior temporal lobe (left inferior longitudinal fasciculus) and the ventral occipital lobe and the orbitofrontal cortex (inferior fronto-occipital fasciculus) (Lebel et al., 2013; Rimrodt, Peterson, Denckla, Kaufmann, \& Cutting, 2010). Importantly, most of the above studies found significant relations between diffusion weighted measures and diverse reading related skills.

DTI studies in children yielded comparable results to the studies in adults. Studies in typically developing children (Beaulieu et al., 2005; Dougherty et al., 2007; Gullick \& Booth, 2014; Nagy, Westerberg, \& Klingberg, 2004) have found a positive relation between diffusivity measures in the posterior limb of the left internal capsule and word identification scores (Beaulieu et al., 2005), callosal fibers that connect the temporal lobes and phonological awareness (Dougherty et al., 2007), and a left temporal lobe region and word reading speed (Nagy et al., 2004). Similar to the studies with adults, studies comparing atypical (poor readers and dyslexics) and typical readers found between group differences in the arcuate and superior longitudinal fasciculus (Carter et al., 2009; Deutsch et al., 2005; Hoeft et al., 2011; Rimrodt et al., 2010; Saygin et al., 2013; Yeatman et al., 2011; Yeatman, Dougherty, Ben-Shachar, \& Wandell, 2012), the anterior and posterior limb of the left internal capsule and corona radiata (Niogi \& McCandliss, 2006; Odegard, Farris, Ring, McColl, \& Black, 2009), the corpus callosum (Hasan et al., 2012; Odegard et al., 2009), and the left inferior longitudinal fasciculus and inferior fronto-occipital fasciculus (Yeatman et al., 2012).

In chapter 5 of this thesis, we investigated differences in fractional anisotropy (FA) between 8-10 year old Dutch typical and dyslexic readers. We found larger FA values in right thalmico-cortical connections of the dyslexic children and these values scaled with individual differences in reading (dys)fluency. As these thalamico-cortical projections could project to sensory-motor areas and lateral prefrontal cortex (Fan, Davis, Anderson, \& Cutting, 2014) the increased FA 
values may represent a prolonged reliance on letter-speech sound connections during reading and compensatory involvement of the working memory.

Another important finding for research of dyslexia is that myelination in the splenium of the corpus callosum and the superior longitudinal fasciculi is highly heritable (Brouwer et al., 2010) which could be connected to the relatively high heritability estimates of reading skills (Carrion-Castillo, Franke, \& Fisher, 2013). Furthermore, genes associated with dyslexia (DYX1C1,DCDC2,and KIAA0319) have been observed to be related to white matter volumes in the left temporo-parietal region of the brain (Darki, Peyrard-Janvid, Matsson, Kere, \& Klingberg, 2012) and specifically DCDC2 deletion was found to be related to disorganization of white matter in the arcuate fasciculus and splenium of corpus callosum (Marino et al., 2014). Interestingly, the rare variants in KIAA0319 and DCDC2 were also found to be associated with the amplitude of a late negativity (300-710 ms) in an oddball paradigm with auditory presented speech syllables (Czamara et al., 2011). Thus, it seems that both electrophysiological functional and anatomical deficits could be related to the same genetic origin. In future work it would be interesting to combine these research lines in the same group of participants to investigate their combined relation to dyslexia and to each other.

In conclusion, the studies we presented here show that neuroimaging can offer a unique insight in both (un)successful reading acquisition and underlying neural reorganization. It could also have a merit in prediction of reading outcomes and further improve diagnostic criteria for developmental dyslexia (Black, Myers, \& Hoeft, 2015). For example, there are studies showing a relation between magneto/electro-physiological responses of the brain on phonological and reading-related tasks and later reading skills (Hämäläinen et al., 2013; Maurer et al., 2009; Rezaie et al., 2011) and a relation of white matter development to reading skills (Yeatman et al., 2012). Thus, a combination of neuroimaging and behavioral measures could help us in the earlier identification of children at risk of dyslexia and the design of more efficient and individually 
tailored treatment programs, which could decrease the number of children with persistent reading problems due to developmental dyslexia.

\section{In this thesis}

The goals of the work presented in this thesis are to: (1) further investigate neural letter-speech sound integration and its relation to reading fluency; (2) identify possible plasticity through cognitive letter-speech sound training for persistent reading dysfluency; (3) investigate functional reading network dynamics during visual word recognition (4) investigate anatomical connectivity in the reading network. To this end we employed electrophysiology and diffusion tensor imaging in 9 year old typical and dyslexic children.

In chapter 2 we use a well-defined MMN paradigm to compare neural letterspeech sound integration of dyslexic and typical readers. In our investigation we go beyond simple group comparisons and also look at the individual differences in fluency and its relation to the electrophysiological data. This approach allows us to observe reduced audiovisual LN effects In both moderately and severely dysfluent dyslexic groups. Interestingly, crossmodal MMN latency scaled with individual differences in reading fluency.

In chapter 3, we go one step further, and retest a group of dyslexic children who followed a special reading training focused on automation of letter-speech sound integration. First, this allowed us to demonstrate a moderate plasticity of the electrical responses of the brain, particularly in the timing of the late negativity. Second, we found that the timing of the MMN may predict reading fluency scores six months later, in addition to its relation with reading fluency at pretest..

In chapter 4 we investigate effective functional connectivity in the reading network during visual word and meaningless symbol recognition in 9 year old dyslexic and typical readers. To achieve this goal we employed a multivariate autoregressive model, directed transfer function (DTF). We observed stronger connectivity from occipital to inferior-temporal sites in typical readers, and 
stronger connectivity towards and from central sites and from frontal to occipito-temporal sites in both dyslexic groups. Furthermore, the group of severely dysfluent dyslexics showed enhanced connectivity in/towards the right hemisphere.

In chapter 5, we examine the properties of white matter anatomical connectivity in 8-10 year old year old typical and dyslexic readers and its relation to reading related skills. We detected 20 white matter tracts using deterministic tractography and extracted fractional anisotropy (FA) values. Our results showed increased fractional anisotropy in right thalamic radiation in dyslexic children, that scaled negatively with reading fluency. Furthermore, typical readers showed a tendency towards larger FA values along multiple tracts in the right hemisphere, and especially in the right uncinate fasciculus.

\section{References}

Al Otaiba, S., \& Fuchs, D. (2002). Characteristics of Children Who Are Unresponsive to Early Literacy Intervention: A Review of the Literature. Remedial and Special Education, 23(5), 300-316. doi:10.1177/07419325020230050501

Alexander, A. W., \& Slinger-Constant, A.-M. (2004). Current status of treatments for dyslexia: critical review. Journal of Cbild Neurology, 19(10), 744-758. doi:10.1177/08830738040190100401

Aravena, S., Snellings, P., Tijms, J., \& van der Molen, M. W. W. (2013). A lab-controlled simulation of a letter-speech sound binding deficit in dyslexia. Journal of Experimental Child Psychology, 115(4), 691-707. doi:10.1016/j.jecp.2013.03.009

Arns, M., Peters, S., Breteler, R., \& Verhoeven, L. (2007). Different brain activation patterns in dyslexic children: evidence from EEG power and coherence patterns for the doubledeficit theory of dyslexia. Journal of Integrative Neuroscience, 6(1), 175-90. Retrieved from http://www.ncbi.nlm.nih.gov/pubmed/17472228

Baart, M., De Boer-Schellekens, L., \& Vroomen, J. (2012). Lipread-induced phonetic recalibration in dyslexia. Acta Psychologica, 140(1), 91-95. doi:10.1016/j.actpsy.2012.03.003

Baccalá, L. a, \& Sameshima, K. (2001). Partial directed coherence: a new concept in neural structure determination. Biological Cybernetics, 84(6), 463-74. Retrieved from http://www.ncbi.nlm.nih.gov/pubmed/11417058

Baron, J., \& Thurston, I. (1973). An analysis of the world-superiority effect. Cognitive Psychology, $4(2), 207-228$.

Beaulieu, C. (2002). The basis of anisotropic water diffusion in the nervous system - A technical review. NMR in Biomedicine, 15, 435-455. doi:10.1002/nbm.782

Beaulieu, C., Plewes, C., Paulson, L. A., Roy, D., Snook, L., Concha, L., \& Phillips, L. (2005). Imaging brain connectivity in children with diverse reading ability. NeuroImage, 25, 1266- 
1271. doi:10.1016/j.neuroimage.2004.12.053

Bishop, D. V. M. (2006). Dyslexia: What's the problem? Developmental Science, 9, 256-257.

Black, J. M., Myers, C. A., \& Hoeft, F. (2015). The Utility of Neuroimaging Studies for Informing Educational Practice and Policy in Reading Disorders. New Directions for Cbild and Adolescent Development, 2015(147), 49-56. doi:10.1002/cad.20086

Blau, V., Reithler, J., van Atteveldt, N., Seitz, J., Gerretsen, P., Goebel, R., \& Blomert, L. (2010). Deviant processing of letters and speech sounds as proximate cause of reading failure: a functional magnetic resonance imaging study of dyslexic children. Brain: A Journal of Neurology, 133(Pt 3), 868-79. doi:10.1093/brain/awp308

Blau, V., van Atteveldt, N., Ekkebus, M., Goebel, R., \& Blomert, L. (2009). Reduced neural integration of letters and speech sounds links phonological and reading deficits in adult dyslexia. Current Biology : CB, 19(6), 503-8. doi:10.1016/j.cub.2009.01.065

Blinowska, K. J., Kuś, R., \& Kamiński, M. (2004). Granger causality and information flow in multivariate processes. Physical Review E, 70(5), 050902. doi:10.1103/PhysRevE.70.050902

Blomert, L. (2005). Dyslexie in Nederland. Amsterdam: Uitgeverij Nieunesijds. Amsterdam: Uitgeverij Nieuwezijds. Retrieved from http://www.boomtestuitgevers.nl/upload/Dyslexie_in_Nederland_Leo_Blomert.pdf

Blomert, L. (2011). The neural signature of orthographic-phonological binding in successful and failing reading development. NeuroImage, 57(3), 695-703. doi:10.1016/j.neuroimage.2010.11.003

Blomert, L., \& Froyen, D. (2010). Multi-sensory learning and learning to read. International Journal of Psychophysiology : Official Journal of the International Organization of Psychophysiology, 77(3), 195204. doi:10.1016/j.ijpsycho.2010.06.025

Blomert, L., \& Vaessen, A. A. (2009). 3DM Differential diagnostics for dyslexia: Cognitive analysis of reading and spelling. Amsterdam: Boom Test Publishers.

Blomert, L., \& Willems, G. (2010). Is there a causal link from a phonological awareness deficit to reading failure in children at familial risk for dyslexia? Dyslexia, 16(4), 300-317. doi:10.1002/dys

Bonte, M. L., \& Blomert, L. (2004). Developmental dyslexia: ERP correlates of anomalous phonological processing during spoken word recognition. Brain Research. Cognitive Brain Research, 21(3), 360-76. doi:10.1016/j.cogbrainres.2004.06.010

Brem, S., Bach, S., Kucian, K., Guttorm, T. K., Martin, E., Lyytinen, H., ... Richardson, U. (2010). Brain sensitivity to print emerges when children learn letter-speech sound correspondences. Proceedings of the National Academy of Sciences of the United States of America, 107(17), 7939-44. doi:10.1073/pnas.0904402107

Brennan, C., Cao, F., Pedroarena-Leal, N., McNorgan, C., \& Booth, J. R. (2013). Reading acquisition reorganizes the phonological awareness network only in alphabetic writing systems. Human Brain Mapping, 34(12), 3354-68. doi:10.1002/hbm.22147

Brouwer, R. M., Mandl, R. C. W., Peper, J. S., van Baal, G. C. M., Kahn, R. S., Boomsma, D. I., \& Hulshoff Pol, H. E. (2010). Heritability of DTI and MTR in nine-year-old children. NeuroImage, 53(3), 1085-1092. doi:10.1016/j.neuroimage.2010.03.017

Bruck, M. (1990). Word-recognition skills of adults with childhood diagnoses of dyslexia. Developmental Psychology, 26(3), 439-454. doi:10.1037/0012-1649.26.3.439

Cao, F., Bitan, T., \& Booth, J. R. (2008). Effective brain connectivity in children with reading difficulties during phonological processing. Brain and Language, 107(2), 91-101. 


\section{General introduction}

doi:10.1016/j.bandl.2007.12.009

Carrion-Castillo, A., Franke, B., \& Fisher, S. E. (2013). Molecular genetics of dyslexia: an overview. Dyslexia (Chichester, England), 19(4), 214-40. doi:10.1002/dys.1464

Carter, J. C., Lanham, D. C., Cutting, L. E., Clements-Stephens, A. M., Chen, X., Hadzipasic, M., ... Kaufmann, W. E. (2009). A dual DTI approach to analyzing white matter in children with dyslexia. Psychiatry Research - Neuroimaging, 172(3), 215-219. doi:10.1016/j.pscychresns.2008.09.005

Castro-Caldas, A., Petersson, K. M., Reis, A., Stone-Elander, S., \& Ingvar, M. (1998). The illiterate brain. Learning to read and write during childhood influences the functional organization of the adult brain. Brain, 121, 1053-1063. doi:10.1093/brain/121.6.1053

Cheour, M., Korpilahti, P., Martynova, O., \& Lang, a H. (2001). Mismatch negativity and late discriminative negativity in investigating speech perception and learning in children and infants. Audiology \& Neuro-Otology, 6(1), 2-11. doi:46804

Collins, J., \& Blot, R. K. (2003). Literacy and Literacies (Texts, Power, and Identity). Cambridge: Cambridge University Press.

Compton, D. L., Miller, A. C., Elleman, A. M., \& Steacy, L. M. (2014). Have We Forsaken Reading Theory in the Name of "Quick Fix" Interventions for Children With Reading Disability? Scientific Studies of Reading, 18(1), 55-73. doi:10.1080/10888438.2013.836200

Cone, N. E., Burman, D. D., Bitan, T., Bolger, D. J., \& Booth, J. R. (2008). Developmental changes in brain regions involved in phonological and orthographic processing during spoken language processing. NeuroImage, 41(2), 623-635. doi:10.1016/j.neuroimage.2008.02.055

Csépe, V. (2003). Auditory event-related potentials in studying developmental dyslexia. In V. Csépe (Ed.), Dyslexia: Different brain, different behavior (pp. 81-112). New York, NY: Kluwer Academic Publishers - Plenum Publishers.

Czamara, D., Bruder, J., Becker, J., Bartling, J., Hoffmann, P., Ludwig, K. U., ... Schulte-Körne, G. (2011). Association of a rare variant with mismatch negativity in a region between KIAA0319 and DCDC2 in dyslexia. Behavior Genetics, 41(1), 110-9. doi:10.1007/s10519010-9413-6

Darki, F., Peyrard-Janvid, M., Matsson, H., Kere, J., \& Klingberg, T. (2012). Three dyslexia susceptibility genes, DYX1C1, DCDC2, and KIAA0319, affect temporo-parietal white matter structure. Biological Psychiatry, 72(8), 671-676. doi:10.1016/j.biopsych.2012.05.008

de Jong, P. F., \& van der Leij, A. (2003). Developmental changes in the manifestation of a phonological deficit in dyslexic children learning to read a regular orthography. Journal of Educational Psychology, 95(1), 22-40. doi:10.1037/0022-0663.95.1.22

Dehaene, S., Cohen, L., Sigman, M., \& Vinckier, F. (2005). The neural code for written words: A proposal. Trends in Cognitive Sciences, 9(7), 335-341. doi:10.1016/j.tics.2005.05.004

Dehaene, S., Pegado, F., Braga, L. W., Ventura, P., Nunes Filho, G., Jobert, A., ... Cohen, L. (2010). How learning to read changes the cortical networks for vision and language. Science (New York, N.Y.), 330(6009), 1359-64. doi:10.1126/science.1194140

Deutsch, G. K., Dougherty, R. F., Bammer, R., Siok, W. T., Gabrieli, J. D. E., \& Wandell, B. (2005). Children's reading performance is correlated with white matter structure measured by diffusion tensor imaging. Cortex; a Journal Devoted to the Study of the Nervous System and Behavior, 41, 354-363. doi:10.1016/S0010-9452(08)70272-7

Dhar, M., Been, P. H., Minderaa, R. B., \& Althaus, M. (2010). Reduced interhemispheric 
coherence in dyslexic adults. Cortex, 46(6), 794-798. doi:10.1016/j.cortex.2009.09.006

Dijkstra, T., Roelofs, A., \& Fieuws, S. (1995). Orthographic effects on phoneme monitoring. Canadian Journal of Experimental Psychology, 49(2), 264-271.

Dougherty, R. F., Ben-Shachar, M., Deutsch, G. K., Hernandez, A., Fox, G. R., \& Wandell, B. a. (2007). Temporal-callosal pathway diffusivity predicts phonological skills in children. Proceedings of the National Academy of Sciences of the United States of America, 104(20), 85568561. doi:10.1073/pnas.0608961104

Dukleth Johnson, D. E., \& Lee Swanson, H. (2011). Cognitive Characteristics of TreatmentResistant Children With Reading Disabilities: A Retrospective Study. Journal of Psychoeducational Assessment, 29, 137-149. doi:10.1177/0734282910380189

Ehri, L. C. (2005). Learning to Read Words: Theory, Findings, and Issues. Scientific Studies of Reading, 9(2), 167-188. doi:10.1207/s1532799xssr0902_4

Ehri, L. C., \& Wilce, L. (1985). Movement into reading: Is the first stage of printed word learning visual or phonetic? Reading Research Quarterly, 20(2), 163-179. doi:10.2307/747753

Engel, a K., Fries, P., \& Singer, W. (2001). Dynamic predictions: oscillations and synchrony in top-down processing. Nature Reviens. Neuroscience, 2(October), 704-716. doi: $10.1038 / 35094565$

Fan, Q., Davis, N., Anderson, A. W., \& Cutting, L. E. (2014). Thalamo-cortical connectivity: what can diffusion tractography tell us about reading difficulties in children? Brain Connectivity, 4(6), 428-39. doi:10.1089/brain.2013.0203

Fraga González, G., Van der Molen, M. J. W., Žarić, G., Bonte, M., Tijms, J., Blomert, L., ... van der Molen, M. W. (n.d.). Graph Analysis of EEG Resting State Functional Networks in Dyslexic and Typically Reading Children.

Fraga González, G., Žarić, G., Tijms, J., Bonte, M., Blomert, L., Leppänen, P. H. T., \& van der Molen, M. W. (n.d.). Visual ERP Changes in Dyslexia Associated with Increasing Reading Fluency.

Fraga González, G., Žarić, G., Tijms, J., Bonte, M., Blomert, L., \& van der Molen, M. W. (2014). Brain-potential analysis of visual word recognition in dyslexics and typically reading children. Frontiers in Human Neuroscience, 8(June), 1-14. doi:10.3389/fnhum.2014.00474

Fraga González, G., Žarić, G., Tijms, J., Bonte, M., Blomert, L., \& van der Molen, M. W. (2015). A Randomized Controlled Trial on The Beneficial Effects of Training Letter-Speech Sound Integration on Reading Fluency in Children with Dyslexia. PloS One, 10(12), e0143914. doi:10.1371/journal.pone.0143914

Froyen, D., Bonte, M. L., van Atteveldt, N., \& Blomert, L. (2009). The long road to automation: neurocognitive development of letter-speech sound processing. Journal of Cognitive Neuroscience, 21(3), 567-80. doi:10.1162/jocn.2009.21061

Froyen, D., van Atteveldt, N., \& Blomert, L. (2010). Exploring the Role of Low Level Visual Processing in Letter-Speech Sound Integration: A Visual MMN Study. Frontiers in Integrative Neuroscience, 4(April), 9. doi:10.3389/fnint.2010.00009

Froyen, D., Van Atteveldt, N., Bonte, M., \& Blomert, L. (2008). Cross-modal enhancement of the MMN to speech-sounds indicates early and automatic integration of letters and speech-sounds. Neuroscience Letters, 430(1), 23-8. doi:10.1016/j.neulet.2007.10.014

Froyen, D., Willems, G., \& Blomert, L. (2011). Evidence for a specific cross-modal association deficit in dyslexia: an electrophysiological study of letter-speech sound processing. Developmental Science, 14(4), 635-48. doi:10.1111/j.1467-7687.2010.01007.x 


\section{General introduction}

Frye, R. E., Hasan, K., Xue, L., Strickland, D., Malmberg, B., Liederman, J., \& Papanicolaou, A. (2008). Splenium microstructure is related to two dimensions of reading skill. Neuroreport, 19(16), 1627-1631. doi:10.1097/WNR.0b013e328314b8ee

Frye, R. E., Liederman, J., Hasan, K. M., Lincoln, A., Malmberg, B., McLean, J., \& Papanicolaou, A. (2011). Diffusion tensor quantification of the relations between microstructural and macrostructural indices of white matter and reading. Human Brain Mapping, 32, 1220-1235. doi:10.1002/hbm. 21103

Gabrieli, J. D. E. (2009). Dyslexia: a new synergy between education and cognitive neuroscience. Science (New York, N.Y.), 325(5938), 280-3. doi:10.1126/science.1171999

Groen, M. A., \& Jesse, A. (2013). Audiovisual Speech Perception in Children and Adolescents With Developmental Dyslexia: No Deficit With McGurk Stimuli. In Auditory-Visual Speech Processing (AVSP) (pp. 77-80). Annecy, France. Retrieved from http://avsp2013.loria.fr/proceedings/papers/paper_49.pdf

Gullick, M. M., \& Booth, J. R. (2014). Individual Differences in Cross-modal Brain Activity Predict Arcuate Fasciculus Connectivity in Developing Readers. Journal of Cognitive Neuroscience, 1-16. doi:10.1162/jocn_a_00581

Hahn, N., Foxe, J. J., \& Molholm, S. (2014). Impairments of multisensory integration and crosssensory learning as pathways to dyslexia. Neuroscience \& Biobehavioral Reviews, 47, 384-392. doi:10.1016/j.neubiorev.2014.09.007

Hämäläinen, J. a, Guttorm, T. K., Richardson, U., Alku, P., Lyytinen, H., \& Leppänen, P. H. T. (2013). Auditory event-related potentials measured in kindergarten predict later reading problems at school age. Developmental Neuropsychology, 38(8), 550-66. doi:10.1080/87565641.2012.718817

Harrar, V., Tammam, J., Pérez-Bellido, A., Pitt, A., Stein, J., \& Spence, C. (2014). Multisensory integration and attention in developmental dyslexia. Current Biology, 24(5), 531-535. doi:10.1016/j.cub.2014.01.029

Hasan, K. M., Molfese, D. L., Walimuni, I. S., Stuebing, K. K., Papanicolaou, A. C., Narayana, P. a., \& Fletcher, J. M. (2012). Diffusion tensor quantification and cognitive correlates of the macrostructure and microstructure of the corpus callosum in typically developing and dyslexic children. NMR in Biomedicine, 25(February), 1263-1270. doi:10.1002/nbm.2797

Hatcher, P. J., Hulme, C., Miles, J. N. V, Carroll, J. M., Hatcher, J., Gibbs, S., ... Snowling, M. J. (2006). Efficacy of small group reading intervention for beginning readers with readingdelay: a randomised controlled trial. Journal of Child Psychology and Psychiatry, and Allied Disciplines, 47(8), 820-7. doi:10.1111/j.1469-7610.2005.01559.x

Hein, G., Doehrmann, O., Müller, N. G., Kaiser, J., Muckli, L., \& Naumer, M. J. (2007). Object familiarity and semantic congruency modulate responses in cortical audiovisual integration areas. The Journal of Neuroscience : The Official Journal of the Society for Neuroscience, 27(30), 78817887. doi:10.1523/JNEUROSCI.1740-07.2007

Hintikka, S., Landerl, K., Aro, M., \& Lyytinen, H. (2008). Training reading fluency: Is it important to practice reading aloud and is generalization possible? Annals of Dyslexia, 58(1), 59-79. doi:10.1007/s11881-008-0012-7

Hoeft, F., McCandliss, B. D., Black, J. M., Gantman, A., Zakerani, N., Hulme, C., ... Gabrieli, J. D. E. (2011). Neural systems predicting long-term outcome in dyslexia. Proceedings of the National Academy of Sciences of the United States of America, 108(1), 361-6. doi:10.1073/pnas.1008950108

Hohn, W. E., \& Ehri, L. C. (1983). Do Alphabet Letters Help Prereaders Acquire Phonemic 
Segmentation Skill ? Journal of Educational Psychology, 75(5), 752-762.

Hommet, C., Vidal, J., Roux, S., Blanc, R., Barthez, M. A., De Becque, B., ... Gomot, M. (2009). Topography of syllable change-detection electrophysiological indices in children and adults with reading disabilities. Neuropsychologia, 47(3), 761-70.

doi:10.1016/j.neuropsychologia.2008.12.010

Jobard, G., Crivello, F., \& Tzourio-Mazoyer, N. (2003). Evaluation of the dual route theory of reading: A metanalysis of 35 neuroimaging studies. NeuroImage, 20, 693-712. doi:10.1016/S1053-8119(03)00343-4

Johansen-Berg, H., \& Behrens, T. E. J. (2009). Diffusion MRI (First.). Elsevier Inc.

Johnston, F. R. (2001). Spelling Exceptions: Problems or Possibilities? The Reading Teacher, 54(4), 372-378. Retrieved from http://www.jstor.org/stable/20204922

Katzir, T., Kim, Y., Wolf, M., O’Brien, B., Kennedy, B., Lovett, M., \& Morris, R. (2006). Reading fluency: the whole is more than the parts. Annals of Dyslexia, 56(1), 51-82. doi:10.1007/s11881-006-0003-5

Katzir, T., Kim, Y.-S. Y.-S., Wolf, M., Morris, R., \& Lovett, M. W. (2008). The Varieties of Pathways to Dysfluent Reading: Comparing Subtypes of Children With Dyslexia at Letter, Word, and Connected Text Levels of Reading. Journal of Learning Disabilities, 41(1), 47-66. doi: $10.1177 / 0022219407311325$

Klingberg, T., Hedehus, M., Temple, E., Salz, T., Gabrieli, J. D., Moseley, M. E., \& Poldrack, R. a. (2000). Microstructure of temporo-parietal white matter as a basis for reading ability: evidence from diffusion tensor magnetic resonance imaging. Neuron, 25(2), 493-500. Retrieved from http://www.ncbi.nlm.nih.gov/pubmed/10719902

Landerl, K., \& Wimmer, H. (2008). Development of word reading fluency and spelling in a consistent orthography: An 8-year follow-up. Journal of Educational Psychology, 100(1), 150161. doi:10.1037/0022-0663.100.1.150

Lebel, C., Shaywitz, B. A., Holahan, J., Shaywitz, S. E., Marchione, K., \& Beaulieu, C. (2013). Diffusion tensor imaging correlates of reading ability in dysfluent and non-impaired readers. Brain and Language, 125(2), 215-222. doi:10.1016/j.bandl.2012.10.009

Leinonen, S., Müller, K., Leppänen, P. H. T., Aro, M., Ahonen, T., \& Lyytinen, H. (2001). Heterogeneity in adult dyslexic readers : Relating processing skills to the speed and accuracy of oral text reading *. Reading and Writing, 14(3-4), 265-296. doi:10.1023/A:1011117620895

Levy, B. A., Abello, B., \& Lysynchuk, L. (1997). Transfer from Word Training to Reading in Context: Gains in Reading Fluency and Comprehension. Learning Disability Quarterly, 20(3), 173-188. doi:10.2307/1511307

Liberman, I. Y., Shankweiler, D., Fischer, F. W., \& Carter, B. (1974). Explicit syllable and phoneme segmentation in the young child. Journal of Experimental Child Psychology, 18(1074), 201-212. doi:10.1016/0022-0965(74)90101-5

Lovett, M. ., Barron, R. ., \& Benson, N. J. (2003). Effective remediation of word identification and decoding difficulties in school-age children with reading disabilities. Handbook of Learning Disabilities, 273-292.

Luck, S. J. (2005). An introduction to the event-related potential technique. Cambridge, MA: The MIT Press.

Lyon, G. R., Shaywitz, S. E., \& Shaywitz, B. A. (2003). A definition of dyslexia. Annals of Dyslexia, 53(1), 1-14. doi:10.1007/s11881-003-0001-9 


\section{General introduction}

Maisog, J. M., Einbinder, E. R., Flowers, D. L., Turkeltaub, P. E., \& Eden, G. F. (2008). A metaanalysis of functional neuroimaging studies of dyslexia. Annals of the New York. Academy of Sciences, 1145, 237-259. doi:10.1196/annals.1416.024

Mann, V., \& Wimmer, H. (2002). Phoneme awareness and pathways into literacy: A comparison of German and American children. Reading and Writing: An Interdisciplinary Journal, 15(7/8), 653-682. doi:10.1023/A:1020984704781

Marino, C., Scifo, P., Della Rosa, P. a., Mascheretti, S., Facoetti, A., Lorusso, M. L., ... Perani, D. (2014). The DCDC2/intron 2 deletion and white matter disorganization: Focus on developmental dyslexia. Cortex, 57, 227-243. doi:10.1016/j.cortex.2014.04.016

Marosi, E., Harmony, T., Becker, J., Reyes, a, Bernal, J., Fernández, T., ... Guerrero, V. (1995). Electroencephalographic coherences discriminate between children with different pedagogical evaluation. International Journal of Psychophysiology: Official Journal of the International Organization of Psychophysiology, 19(1), 23-32. doi:10.1016/0167-8760(94)00059$\mathrm{N}$

Martin, A., Schurz, M., Kronbichler, M., \& Richlan, F. (2015). Reading in the brain of children and adults: A meta-analysis of 40 functional magnetic resonance imaging studies. Human Brain Mapping, 00(January), n/a-n/a. doi:10.1002/hbm.22749

Maurer, U., Brem, S., Bucher, K., \& Brandeis, D. (2005). Emerging neurophysiological specialization for letter strings. Journal of Cognitive Neuroscience, 17(10), 1532-52. doi:10.1162/089892905774597218

Maurer, U., Brem, S., Kranz, F., Bucher, K., Benz, R., Halder, P., ... Brandeis, D. (2006). Coarse neural tuning for print peaks when children learn to read. NeuroImage, 33(2), 749-58. doi:10.1016/j.neuroimage.2006.06.025

Maurer, U., Bucher, K., Brem, S., Benz, R., Kranz, F., Schulz, E., ... Brandeis, D. (2009). Neurophysiology in preschool improves behavioral prediction of reading ability throughout primary school. Biological Psychiatry, 66(4), 341-8. doi:10.1016/j.biopsych.2009.02.031

Maurer, U., Schulz, E., Brem, S., der Mark, S. Van, Bucher, K., Martin, E., \& Brandeis, D. (2011). The development of print tuning in children with dyslexia: evidence from longitudinal ERP data supported by fMRI. NeuroImage, 57(3), 714-22. doi:10.1016/j.neuroimage.2010.10.055

McCandliss, B. D., Cohen, L., \& Dehaene, S. (2003). The visual word form area: expertise for reading in the fusiform gyrus. Trends in Cognitive Sciences, 777), 293-299. doi:10.1016/S13646613(03)00134-7

McMaster, K. L., Fuchs, D., Fuchs, L. S., \& Compton, D. L. (2005). Responding to Nonresponders: An Experimental Field Trial of Identification and Intervention Methods. Exceptional Children, 71(4), 445-463. Retrieved from http://www.questia.com/googleScholar.qst?docId=5009925718

McNorgan, C., Randazzo-Wagner, M., \& Booth, J. R. (2013). Cross-modal integration in the brain is related to phonological awareness only in typical readers, not in those with reading difficulty. Frontiers in Human Neuroscience, 7(July), 388. doi:10.3389/fnhum.2013.00388

Monzalvo, K., \& Dehaene-Lambertz, G. (2013). How reading acquisition changes children's spoken language network. Brain and Language, 127(3), 356-65. doi:10.1016/j.bandl.2013.10.009

Monzalvo, K., Fluss, J., Billard, C., Dehaene, S., \& Dehaene-Lambertz, G. (2012). Cortical networks for vision and language in dyslexic and normal children of variable socio- 
economic status. NeuroImage, 61(1), 258-74. doi:10.1016/j.neuroimage.2012.02.035

Morais, J., Alegria, J., \& Content, A. (1987). The relationships between segmental analysis and alphabetic literacy: An interactive view. Cabiers de Psychologie Cognitive, 7(5), 415-438.

Morais, J., Cary, L., Alegria, J., \& Bertelson, P. (1979). Does awareness of speech as a sequence of phones arise spontaneously? Cognition, 7(4), 323-331. doi:10.1016/0010-0277(79)90020-9

Morais, J., Castro, S. L., Scliar-Cabral, L., Kolinsky, R., \& Content, A. (1987). The effects of literacy on the recognition of dichotic words. The Quarterly Journal of Experimental Psychology. A, Human Experimental Psychology, 39(3), 451-465. doi:10.1080/14640748708401798

Näätänen, R. (2001). The perception of speech sounds by the human brain as reflected by the mismatch negativity (MMN) and its magnetic equivalent (MMNm). Psychophysiology, 38(1), $1-21$.

Näätänen, R., Paavilainen, P., Rinne, T., \& Alho, K. (2007). The mismatch negativity (MMN) in basic research of central auditory processing: a review. Clinical Neurophysiology : Official Journal of the International Federation of Clinical Neurophysiology, 118(12), 2544-90. doi:10.1016/j.clinph.2007.04.026

Nagarajan, S., Mahncke, H., Salz, T., Tallal, P., Roberts, T., \& Merzenich, M. M. (1999). Cortical auditory signal processing in poor readers. Proceedings of the National Academy of Sciences of the United States of America, 96(11), 6483-6488. doi:10.1073/pnas.96.11.6483

Nagy, Z., Westerberg, H., \& Klingberg, T. (2004). Maturation of white matter is associated with the development of cognitive functions during childhood. Journal of Cognitive Neuroscience, 16(Figure 1), 1227-1233. doi:10.1162/0898929041920441

Naumer, M. J., Doehrmann, O., Müller, N. G., Muckli, L., Kaiser, J., \& Hein, G. (2009). Cortical plasticity of audio-visual object representations. Cerebral Cortex, 19(7), 1641-1653. doi: $10.1093 /$ cercor/bhn200

Nazir, T. a, Jacobs, a M., \& O'Regan, J. K. (1998). Letter legibility and visual word recognition. Memory \& Cognition, 26(4), 810-821. doi:10.3758/BF03211400

Nelson, J. R., Benner, G. J., \& Gonzalez, J. (2003). Learner characteristics that influence treatment effectiveness of early literacy interventions: A meta-analytic review. Learning Disabilities Research and Practice, 18(4), 255-267.

New, B., Ferrand, L., Pallier, C., \& Brysbaert, M. (2006). Reexamining the word length effect in visual word recognition: new evidence from the English Lexicon Project. Psychonomic Bulletin \& Review, 13(1), 45-52. doi:10.3758/BF03193811

Niogi, S. N., \& McCandliss, B. D. (2006). Left lateralized white matter microstructure accounts for individual differences in reading ability and disability. Neuropsychologia, 44, 2178-2188. doi:10.1016/j.neuropsychologia.2006.01.011

Nolte, G., Bai, O., Wheaton, L., Mari, Z., Vorbach, S., \& Hallett, M. (2004). Identifying true brain interaction from EEG data using the imaginary part of coherency. Clinical Neurophysiology, 115(10), 2292-2307. doi:10.1016/j.clinph.2004.04.029

Odegard, T. N., Farris, E. a., Ring, J., McColl, R., \& Black, J. (2009). Brain connectivity in nonreading impaired children and children diagnosed with developmental dyslexia. Neuropsychologia, 47, 1972-1977. doi:10.1016/j.neuropsychologia.2009.03.009

Ogawa, S., Lee, T. M., Kay, a R., \& Tank, D. W. (1990). Brain magnetic resonance imaging with contrast dependent on blood oxygenation. Proceedings of the National Academy of Science USA, 87(24), 9868-9872.

Pekkola, J., Laasonen, M., Ojanen, V., Autti, T., Jääskeläinen, I. P., Kujala, T., \& Sams, M. (2006). 


\section{General introduction}

Perception of matching and conflicting audiovisual speech in dyslexic and fluent readers: An fMRI study at 3 T. NeuroImage, 29(3), 797-807. doi:10.1016/j.neuroimage.2005.09.069

Pugh, K. R., Mencl, W. E., Jenner, a R., Katz, L., Frost, S. J., Lee, J. R., ... Shaywitz, B. A. (2001). Neurobiological studies of reaing and reading disability. Journal of Communicative Disorders, 34, 479-492.

Pugh, K. R., Mencl, W. E., Jenner, A. R., Lee, J. R., Katz, L., Frost, S. J., ... Shaywitz, B. A. (2001). Neuroimaging Studies of Reading Development and Reading Disability. Learning Disabilities Research and Practice, 16(4), 240-249. doi:10.1111/0938-8982.00024

Quaglino, V., Bourdin, B., Czternasty, G., Vrignaud, P., Fall, S., Meyer, M. E., ... de Marco, G. (2008). Differences in effective connectivity between dyslexic children and normal readers during a pseudoword reading task: An fMRI study. Neurophysiologie Clinique, 38, 73-82. doi:10.1016/j.neucli.2007.12.007

Ramus, F., \& Szenkovits, G. (2008). What phonological deficit? Quarterly Journal of Experimental Psychology (2006), 61(1), 129-41. doi:10.1080/17470210701508822

Rayner, K., Pollatsek, A., \& Schotter, E. R. (2012). Reading: Word Identification and Eye Movements. In A. Healy (Ed.), Handbook of psychology, volume 4: Experimental psychology (pp. 548-577). Hoboken: Wiley.

Read, C., Zhang, Y. F., Nie, H. Y., \& Ding, B. Q. (1986). The ability to manipulate speech sounds depends on knowing alphabetic writing. Cognition, 24(1-2), 31-44. Retrieved from http://www.ncbi.nlm.nih.gov/pubmed/3791920

Reicher, G. M. (1969). Perceptual recognition as a function of meaninfulness of stimulus material. Journal of Experimental Psychology, 81(2), 275-280. doi:10.1037/h0027768

Rezaie, R., Simos, P. G., Fletcher, J. M., Cirino, P. T., Vaughn, S., \& Papanicolaou, A. C. (2011). Temporo-parietal Brain Activity as a Longitudinal Predictor of Response to Educational Interventions among Middle School Struggling Readers. Journal of the International Neuropsychological Society : JINS, 17(5), 875-885. doi:10.1017/S1355617711000890

Richards, T. L., \& Berninger, V. W. (2008). Abnormal fMRI connectivity in children with dyslexia during a phoneme task: Before but not after treatment. Journal of Neurolinguistics, 21, 294-304. doi:10.1016/j.jneuroling.2007.07.002

Richards, T. L., Stevenson, J., Crouch, J., Johnson, L. C., Maravilla, K., Stock, P., ... Berninger, V. (2008). Tract-based spatial statistics of diffusion tensor imaging in adults with dyslexia. American Journal of Neuroradiology, 29, 1134-1139. doi:10.3174/ajnr.A1007

Richlan, F., Kronbichler, M., \& Wimmer, H. (2009). Functional abnormalities in the dyslexic brain: A quantitative meta-analysis of neuroimaging studies. Human Brain Mapping, 30(10), 3299-3308. doi:10.1002/hbm.20752

Rimrodt, S. L., Peterson, D. J., Denckla, M. B., Kaufmann, W. E., \& Cutting, L. E. (2010). White matter microstructural differences linked to left perisylvian language network in children with dyslexia. Cortex, 46(6), 739-749. doi:10.1016/j.cortex.2009.07.008

Roberts, T. a. (2003). Effects of alphabet-letter instruction on young children's word recognition. Journal of Educational Psychology, 95(1), 41-51. doi:10.1037/0022-0663.95.1.41

Sandak, R., Mencl, W. E., Frost, S. J., \& Pugh, K. R. (2004). The Neurobiological Basis of Skilled and Impaired Reading: Recent Findings and New Directions. Scientific Studies of Reading, 8(3), 273-292. doi:10.1207/s1532799xssr0803_6

Saygin, Z. M., Norton, E. S., Osher, D. E., Beach, S. D., Cyr, a. B., Ozernov-Palchik, O., ... Gabrieli, J. D. E. (2013). Tracking the Roots of Reading Ability: White Matter Volume and 


\section{Chapter 1}

Integrity Correlate with Phonological Awareness in Prereading and Early-Reading Kindergarten Children. Journal of Neuroscience, 33(33), 13251-13258. doi:10.1523/JNEUROSCI.4383-12.2013

Scheltinga, F., van der Leij, a., \& Struiksma, C. (2010). Predictors of Response to Intervention of Word Reading Fluency in Dutch. Journal of Learning Disabilities, 43(3), 212-228. doi:10.1177/0022219409345015

Schlaggar, B. L., \& McCandliss, B. D. (2007). Development of neural systems for reading. Annual Review of Neuroscience, 30, 475-503. doi:10.1146/annurev.neuro.28.061604.135645

Schlögl, A., \& Supp, G. (2006). Analyzing event-related EEG data with multivariate autoregressive parameters. Progress in Brain Research, 159, 135-47. doi:10.1016/S00796123(06)59009-0

Schulte-Körne, G., Deimel, W., Bartling, J., \& Remschmidt, H. (1998). Auditory processing and dyslexia: evidence for a specific speech processing deficit. Neuroreport, 9(2), 337-340.

Schurz, M., Wimmer, H., Richlan, F., Ludersdorfer, P., Klackl, J., \& Kronbichler, M. (2014). Resting-State and Task-Based Functional Brain Connectivity in Developmental Dyslexia. Cerebral Cortex (New York, N.Y. : 1991), 1-13. doi:10.1093/cercor/bhu184

Seidenberg, M. S., \& Tanenhaus, M. K. (1979). Orthographic effects on rhyme monitoring. Journal of Experimental Psychology: Human Learning and Memory. doi:10.1037/02787393.5.6.546

Share, D. L. (1995). Phonological recoding and self-teaching: sine qua non of reading acquisition. Cognition, 55(2), 151-218; discussion 219-26. Retrieved from http://www.ncbi.nlm.nih.gov/pubmed/7789090

Shaywitz, B. A., Shaywitz, S. E., Pugh, K. R., Mencl, W. E., Fulbright, R. K., Skudlarski, P., .. Gore, J. C. (2002). Disruption of posterior brain systems for reading in children with developmental dyslexia. Biological Psychiatry, 52(2), 101-110. doi:10.1016/S00063223(02)01365-3

Shaywitz, B. A., Skudlarski, P., Holahan, J. M., Marchione, K. E., Constable, R. T., Fulbright, R. K., ... Shaywitz, S. E. (2007). Age-related changes in reading systems of dyslexic children. Annals of Neurology, 61, 363-370. doi:10.1002/ana.21093

Shaywitz, S. E., Morris, R., \& Shaywitz, B. A. (2008). The education of dyslexic children from childhood to young adulthood. Annual Review of Psychology, 59, 451-75. doi:10.1146/annurev.psych.59.103006.093633

Shaywitz, S. E., \& Shaywitz, B. A. (2005). Dyslexia (specific reading disability). Biological Psychiatry, 57(11), 1301-9. doi:10.1016/j.biopsych.2005.01.043

Shaywitz, S. E., \& Shaywitz, B. A. (2008). Paying attention to reading: the neurobiology of reading and dyslexia. Development and Psychopathology, 20(4), 1329-49. doi:10.1017/S0954579408000631

Shaywitz, S. E., Shaywitz, B. A., Fulbright, R. K., Skudlarski, P., Mencl, W. E., Constable, R. T., ... Gore, J. C. (2003). Neural systems for compensation and persistence: Young adult outcome of childhood reading disability. Biological Psychiatry, 54(03), 25-33. doi:10.1016/S0006-3223(03)01836-X

Shiota, M., Koeda, T., \& Takeshita, K. (2000). Cognitive and neurophysiological evaluation of Japanese dyslexia. Brain and Development, 22(7), 421-426. doi:10.1016/S03877604(00)00167-4

Simos, P. G., Breier, J. I., Fletcher, J. M., Foorman, B. R., Castillo, E. M., \& Papanicolaou, A. C. 


\section{General introduction}

(2002). Brain mechanisms for reading words and pseudowords: an integrated approach. Cerebral Cortex (New York, N.Y. : 1991), 12, 297-305. doi:10.1093/cercor/12.3.297

Snowling, M. J. (1980). The development of grapheme-phoneme correspondence in normal and dyslexic readers. Journal of Experimental Child Psychology, 29(2), 294-305. Retrieved from http://www.ncbi.nlm.nih.gov/pubmed/7365427

Snowling, M. J. (1998). Dyslexia as a Phonological Deficit: Evidence and Implications. Child Psychology and Psychiatry Review, 3(1), 4-11. doi:10.1017/S1360641797001366

Snowling, M. J. (2013). Early identification and interventions for dyslexia: a contemporary view. Journal of Research in Special Educational Needs, 13(1), 7-14. doi:10.1111/j.14713802.2012.01262.x

Stanberry, L. I., Richards, T. L., Berninger, V. W., Nandy, R. R., Aylward, E. H., Maravilla, K. R., ... Cordes, D. (2006). Low-frequency signal changes reflect differences in functional connectivity between good readers and dyslexics during continuous phoneme mapping. Magnetic Resonance Imaging, 24, 217-229. doi:10.1016/j.mri.2005.12.006

Steinbrink, C., Vogt, K., Kastrup, a., Müller, H. P., Juengling, F. D., Kassubek, J., \& Riecker, a. (2008). The contribution of white and gray matter differences to developmental dyslexia: Insights from DTI and VBM at 3.0 T. Neuropsychologia, 46, 3170-3178. doi:10.1016/j.neuropsychologia.2008.07.015

Thaler, V., Ebner, E. M., Wimmer, H., \& Landerl, K. (2004). Training reading fluency in dysfluent readers with high reading accuracy: word specific effects but low transfer to untrained words. Annals of dyslexia (Vol. 54). doi:10.1007/s11881-004-0005-0

Thiebaut De Schotten, M., Cohen, L., Amemiya, E., Braga, L. W., \& Dehaene, S. (2014). Learning to read improves the structure of the arcuate fasciculus. Cerebral Cortex, 24, 989995. doi:10.1093/cercor/bhs383

Tijms, J. (2004). A Process-Oriented Evaluation of a Computerised Treatment for Dyslexia. Educational Psychology, 24(6), 767-791. doi:10.1080/0144341042000271719

Tijms, J. (2007). The Development of Reading Accuracy and Reading Rate during Treatment of Dyslexia. Educational Psychology, 27(2), 273-294. doi:10.1080/01443410601066800

Tijms, J. (2011). Effectiveness of computer-based treatment for dyslexia in a clinical care setting: outcomes and moderators. Educational Psychology, 31(7), 873-896.

Torgesen, J. K. (2000). Individual differences in response to early interventions in reading: The lingering problem of treatment resisters. Learning Disabilities Research \& Practice. doi:10.1207/SLDRP1501_6

Torgesen, J. K., Alexander, a W., Wagner, R. K., Rashotte, C. a, Voeller, K. K., \& Conway, T. (2001). Intensive remedial instruction for children with severe reading disabilities: immediate and longterm outcomes from two instructional approaches. Journal of learning disabilities (Vol. 34). doi:10.1177/002221940103400104

Toste, J. R., Compton, D. L., Fuchs, D., Fuchs, L. S., Gilbert, J. K., Cho, E., ... Bouton, B. D. (2014). Understanding Unresponsiveness to Tier 2 Reading Intervention: Exploring the Classification and Profiles of Adequate and Inadequate Responders in First Grade. Learning Disability Quarterly, 37, 192-203. doi:10.1177/0731948713518336

Tran, L., Sanchez, T., Arellano, B., \& Lee Swanson, H. (2011). A meta-analysis of the RTI literature for children at risk for reading disabilities. Journal of Learning Disabilities, 44, 283 295. doi:10.1177/0022219410378447

Undheim, A. M., \& Sund, A. M. (2008). Psychosocial factors and reading difficulties: Students 
with reading difficulties drawn from a representative population sample. Scandinavian Journal of Psychology, 49(4), 377-384. doi:10.1111/j.1467-9450.2008.00661.x

Undheim, A. M., Wichstrøm, L., \& Sund, A. M. (2011). Emotional and Behavioral Problems Among School Adolescents With and Without Reading Difficulties as Measured by the Youth Self-Report: A One-Year Follow-Up Study. Scandinavian Journal of Educational Research, 55(3), 291-305. doi:10.1080/00313831.2011.576879

UNESCO. (2005). Education for all: Literacy for life. Paris: UNESCO publishing.

Vaessen, A., Bertrand, D., Tóth, D., Csépe, V., Faísca, L., Reis, A., \& Blomert, L. (2010). Cognitive development of fluent word reading does not qualitatively differ between transparent and opaque orthographies. Journal of Educational Psychology, 102(4), 827-842. doi:10.1037/a0019465

Vaessen, A., \& Blomert, L. (2010). Long-term cognitive dynamics of fluent reading development. Journal of Experimental Child Psychology, 105(3), 213-31. doi:10.1016/j.jecp.2009.11.005

van Atteveldt, N., \& Ansari, D. (2014). How symbols transform brain function: A review in memory of Leo Blomert. Trends in Neuroscience and Education, 1-5. doi:10.1016/j.tine.2014.04.001

van Atteveldt, N., Formisano, E., Goebel, R., \& Blomert, L. (2004). Integration of letters and speech sounds in the human brain. Neuron, 43(2), 271-82. doi:10.1016/j.neuron.2004.06.025

van der Mark, S., Bucher, K., Maurer, U., Schulz, E., Brem, S., Buckelmüller, J., ... Brandeis, D. (2009). Children with dyslexia lack multiple specializations along the visual word-form (VWF) system. NeuroImage, 47(4), 1940-1949. doi:10.1016/j.neuroimage.2009.05.021

van der Mark, S., Klaver, P., Bucher, K., Maurer, U., Schulz, E., Brem, S., ... Brandeis, D. (2011). The left occipitotemporal system in reading: Disruption of focal fMRI connectivity to left inferior frontal and inferior parietal language areas in children with dyslexia. NeuroImage, 54(3), 2426-2436. doi:10.1016/j.neuroimage.2010.10.002

Vandermosten, M., Boets, B., Poelmans, H., Sunaert, S., Wouters, J., \& Ghesquière, P. (2012). A tractography study in dyslexia: Neuroanatomic correlates of orthographic, phonological and speech processing. Brain, 135, 935-948. doi:10.1093/brain/awr363

Vandermosten, M., Boets, B., Wouters, J., \& Ghesquière, P. (2012). A qualitative and quantitative review of diffusion tensor imaging studies in reading and dyslexia. Neuroscience and Biobehavioral Reviews, 36(6), 1532-1552. doi:10.1016/j.neubiorev.2012.04.002

Vandermosten, M., Poelmans, H., Sunaert, S., Ghesquière, P., \& Wouters, J. (2013). White matter lateralization and interhemispheric coherence to auditory modulations in normal reading and dyslexic adults. Neuropsychologia, 51(11), 2087-2099.

doi:10.1016/j.neuropsychologia.2013.07.008

Varela, F., Lachaux, J. P., Rodriguez, E., \& Martinerie, J. (2001). The brainweb: phase synchronization and large-scale integration. Nature Reviews. Neuroscience, 2(4), 229-239. doi: $10.1038 / 35067550$

Welcome, S. E., \& Joanisse, M. F. (2014). Individual differences in white matter anatomy predict dissociable components of reading skill in adults. NeuroImage, 96C, 261-275. doi:10.1016/j.neuroimage.2014.03.069

Whitney, C., \& Cornelissen, P. (2005). Letter-position encoding and dyslexia. Journal of Research in Reading, 28(3), 274-301. doi:10.1111/j.1467-9817.2005.00270.x

Wimmer, H. (1993). Characteristics of developmental dyslexia in a regular writing system. Applied 


\section{General introduction}

Psycholinguistics, 14(01), 1. doi:10.1017/S0142716400010122

Wimmer, H. (1996). The nonword reading deficit in developmental dyslexia: evidence from children learning to read German. Journal of Experimental Child Psychology, 61(1), 80-90. doi:10.1006/jecp.1996.0004

Wimmer, H., Landerl, K., Linortner, R., \& Hummer, P. (1991). The relationship of phonemic awareness to reading acquisition: more consequence than precondition but still important. Cognition, 40(3), 219-249. doi:10.1016/0010-0277(91)90026-Z

Wimmer, H., \& Schurz, M. (2010). Dyslexia in regular orthographies: manifestation and causation. Dyslexia, 16(4), 283-299. doi:10.1002/dys.411

Wolff, U. (2011). Effects of a randomised reading intervention study: An application of structural equation modelling. In Dyslexia (Vol. 17, pp. 295-311). doi:10.1002/dys.438

Yeatman, J. D., Dougherty, R. F., Ben-Shachar, M., \& Wandell, B. a. (2012). Development of white matter and reading skills. Proceedings of the National Academy of Sciences of the United States of America, 109(44), E3045-53. doi:10.1073/pnas.1206792109

Yeatman, J. D., Dougherty, R. F., Rykhlevskaia, E., Sherbondy, A. J., Deutsch, G. K., Wandell, B. A., \& Ben-Shachar, M. (2011). Anatomical properties of the arcuate fasciculus predict phonological and reading skills in children. Journal of Cognitive Neuroscience, 23(11), 3304-17. doi:10.1162/jocn_a_00061

Žarić, G., Fraga González, G., Tijms, J., van der Molen, M. W., Blomert, L., \& Bonte, M. (2014). Reduced neural integration of letters and speech sounds in dyslexic children scales with individual differences in reading fluency. PloS One, 9(10), e110337. doi:10.1371/journal.pone.0110337

Žarić, G., Fraga González, G., Tijms, J., van der Molen, M. W., Blomert, L., \& Bonte, M. (2015). Crossmodal deficit in dyslexic children: practice affects the neural timing of letter-speech sound integration. Frontiers in Human Neuroscience, 369. doi:10.3389/ fnhum.2015.00369

Ziegler, J. C., \& Ferrand, L. (1998). Orthography shapes the perception of speech: The consistency effect in auditory word recognition. Psychonomic Bulletin \& Review. doi:10.3758/BF03208845

Ziegler, J. C., \& Goswami, U. (2005). Reading acquisition, developmental dyslexia, and skilled reading across languages: a psycholinguistic grain size theory. Psychological Bulletin, 131(1), 3-29. doi:10.1037/0033-2909.131.1.3

Zoccolotti, P., De Luca, M., Di Pace, E., Gasperini, F., Judica, A., \& Spinelli, D. (2005). Word length effect in early reading and in developmental dyslexia. Brain and Language, 93(3), 369373. doi:10.1016/j.bandl.2004.10.010 


\title{
CHAPTER 2
}

\author{
Reduced neural integration of \\ letters and speech sounds in \\ dyslexic children scales with \\ individual differences in \\ reading fluency
}

Corresponding publication:

Žarić, G., Fraga González, G., Tijms, J., van der Molen, M. W., Blomert, L., and Bonte, M. (2014). Reduced neural integration of letters and speech sounds in dyslexic children scales with individual differences in reading fluency. PLoS One 9, e110337. 


\section{Reading Fluency and Cross-Modal ERPs in Dyslexia}

\section{Abstract}

The acquisition of letter-speech sound associations is one of the basic requirements for fluent reading acquisition and its failure may contribute to reading difficulties in developmental dyslexia. Here we investigated eventrelated potential (ERP) measures of letter-speech sound integration in 9-yearold typical and dyslexic readers and specifically test their relation to individual differences in reading fluency. We employed an audiovisual oddball paradigm in typical readers $(n=20)$, dysfluent $(n=18)$ and severely dysfluent $(n=18)$ dyslexic children. In one auditory and two audiovisual conditions the Dutch spoken vowels /a/ and /o/ were presented as standard and deviant stimuli. In audiovisual blocks, the letter 'a' was presented either simultaneously (AV0), or $200 \mathrm{~ms}$ before (AV200) vowel sound onset. Across the three children groups, vowel deviancy in auditory blocks elicited comparable mismatch negativity $(\mathrm{MMN})$ and late negativity (LN) responses. In typical readers, both audiovisual conditions (AV0 and AV200) led to enhanced MMN and LN amplitudes. In both dyslexic groups, the audiovisual LN effects were mildly reduced. Most interestingly, individual differences in reading fluency were correlated with MMN latency in the AV0 condition. A further analysis revealed that this effect was driven by a short-lived MMN effect encompassing only the N1 window in severely dysfluent dyslexics versus a longer MMN effect encompassing both the N1 and P2 windows in the other two groups. Our results confirm and extend previous findings in dyslexic children by demonstrating a deficient pattern of letter-speech sound integration depending on the level of reading dysfluency. These findings underscore the importance of considering individual differences across the entire spectrum of reading skills in addition to group differences between typical and dyslexic readers. 


\section{Introduction}

Though many children learn to read without any problems, $5 \%$ to $10 \%$ of children are affected by developmental dyslexia and never acquire proficient reading skills despite normal cognitive abilities and schooling opportunities (Blomert, 2005; Lyon, Shaywitz, \& Shaywitz, 2003). A lack of reading fluency has been pinpointed as the most persistent and impaired characteristic of developmental dyslexia (Shaywitz, Morris, \& Shaywitz, 2008), although studies also show a large inter-individual variability in the level of reading (dys)fluency (Katzir, Kim, Wolf, Morris, \& Lovett, 2008; Leinonen et al., 2001). A first important step in obtaining reading fluency in alphabetic orthographies, the formation of solid letter-speech sound correspondences, poses an immediate hurdle for beginning dyslexic readers (Blomert, 2011; Ehri, 2005; Share, 1995; Wimmer \& Schurz, 2010). Accordingly, in addition to tackling well-known difficulties in phonological processing, i.e. recognizing and manipulating the sound structure of language (Ramus \& Szenkovits, 2008; Snowling, 1998), many dyslexia interventions contain a condition focused on teaching letterspeech sound correspondences (Aravena, Snellings, Tijms, \& van der Molen, 2013; Bus \& Ijzendoorn, 1999; Tijms \& Hoeks, 2005). In recent years, the neurofunctional basis of the difficulties in building these correspondences has been elucidated by neuroimaging findings showing reduced neural integration of letters and speech sounds in dyslexic children (Blau et al., 2010; Froyen, Willems, \& Blomert, 2011; McNorgan, Randazzo-Wagner, \& Booth, 2013) and adults (Blau, van Atteveldt, Ekkebus, Goebel, \& Blomert, 2009). How this reduced neural integration scales with individual differences in the level of reading (dys)fluency remains an interesting and open question.

In largely transparent languages, accurate letter-speech sound identification and discrimination is typically observed after one year of reading instruction (Blomert \& Vaessen, 2009). However, evidence from neuroimaging studies shows a dissociation between knowing which letters belong to which speech sounds and the automatic neural integration of these associations, with the latter showing a much more protracted period of incremental learning throughout primary school in typical readers (Booth et al., 2001; Froyen, Bonte, 


\section{Reading Fluency and Cross-Modal ERPs in Dyslexia}

van Atteveldt, \& Blomert, 2009). One of the first studies in this domain recorded magnetoencephalography (MEG) in literate Finnish adults and indicated a dynamic process of letter-speech sound integration with audiovisual convergence starting around $225 \mathrm{~ms}$ in bilateral temporal cortices (Raij, Uutela, \& Hari, 2000). Subsequent functional magnetic resonance imaging (fMRI) evidence revealed the involvement of the superior temporal sulcus, as well as auditory cortex (superior temporal gyrus/planum temporale) (van Atteveldt, Formisano, Goebel, \& Blomert, 2004). More specifically, results indicated a modulating influence of letters on speech sounds as a consequence of feedback from the superior temporal sulcus to the superior temporal gyrus, but only if the letter and speech sound were presented simultaneously and in a passive listening paradigm (van Atteveldt, Formisano, Blomert, \& Goebel, 2007; van Atteveldt et al., 2004; van Atteveldt, Formisano, Goebel, \& Blomert, 2007).

Evidence for the protracted developmental time-course of letter-speech sound integration comes from a series of studies measuring electroencephalography (EEG) responses in a passive crossmodal "oddball" paradigm (Froyen et al., 2009; Froyen, Van Atteveldt, Bonte, \& Blomert, 2008; Froyen et al., 2011). In an oddball paradigm, at a latency of $100-200 \mathrm{~ms}$, a mismatch negativity (MMN) response is elicited by a rare sound (deviant, or oddball) that is presented in a sequence of frequent (standard) sounds (Näätänen, 2001). The MMN represents an automatic change detection response sensitive to deviation from traces in auditory short-term memory (Näätänen, Paavilainen, Rinne, \& Alho, 2007; Näätänen, 2001). Multiple experiments have shown that the MMN is sensitive to language-specific speech sounds in adults and in children (Bonte, Poelmans, \& Blomert, 2007; Marie Cheour, Shestakova, Alku, Ceponiene, \& Näätänen, 2002; Cheour-Luhtanen et al., 1995; V Csépe, 1995; Eulitz \& Lahiri, 2004; Kasai et al., 2002; Lohvansuu et al., 2013; Näätänen, 2001; Neuhoff et al., 2012; Winkler et al., 1999), as well as to audiovisual integration (Andres, Oram Cardy, \& Joanisse, 2011; Colin et al., 2002; Froyen et al., 2009, 2008; Mittag, Alho, Takegata, Makkonen, \& Kujala, 2013; Mittag, Takegata, \& Kujala, 2011; Stekelenburg, Vroomen, \& de Gelder, 2004). Furthermore, in school-aged children the speech evoked MMN response may be followed by an additional 
late negativity (Late MMN or LN) in a broader time window from 300 to 700 ms, the amplitude of which decreases in adulthood (M Cheour, Korpilahti, Martynova, \& Lang, 2001; Czamara et al., 2011; Hommet et al., 2009), suggesting fast and automatic discrimination and representation of speech syllables in adults while the developing brain still needs additional processing resources to reach to the same result. In the paradigm of Froyen and colleagues (Froyen et al., 2009, 2008, 2011), in an auditory and two audiovisual experimental blocks, the Dutch speech sound /a/ was presented as the standard stimulus, and the Dutch speech sound /o/ as the deviant stimulus. In the audiovisual blocks, the letter 'a' was presented together with the standard and deviant speech sounds, leading to a double violation: the deviant speech sound /o/ violated the expectation built by both the standard speech sound /a/ and the letter ' $a$ '. In order to investigate the temporal window of integration the letter 'a' was presented either simultaneously, or $200 \mathrm{~ms}$ before speech sound onset. Due to the double violation, literate adults showed a significant enhancement of the MMN response in the audiovisual as compared to auditory blocks, but, reminiscent of the fMRI findings (van Atteveldt, Formisano, Blomert, et al., 2007; van Atteveldt et al., 2004; van Atteveldt, Formisano, Goebel, et al., 2007), only when letters and speech sounds were presented simultaneously, indicating an early and automatic audiovisual integration (Froyen et al., 2008). Using the same paradigm, 8 year-old typically reading children after one year of reading instruction only showed a significant audiovisual enhancement of a late negativity (LN) evoked around $650 \mathrm{~ms}$ after speech sound onset and only when the letter preceded the speech sound, indicating a slow and immature neural integration of letters and speech sounds (Froyen et al., 2009). A more developed, bit still not adult-like pattern was apparent in 11 year-old typically reading children after four years of reading instruction. That is, these more advanced readers showed a cross-modal enhancement of the earlier MMN when the letter preceded the speech sound, and a cross-modal LN enhancement when the letter and speech sound were presented simultaneously (Froyen et al., 2009). Although adults did not show these late letter effects in this passive paradigm using simple speech stimuli (Froyen et al., 2008), orthographic influences on spoken language processing 


\section{Reading Fluency and Cross-Modal ERPs in Dyslexia}

around 400-700 ms have been reported in adults when using a more complex metaphonological task (Lafontaine, Chetail, Colin, Kolinsky, \& Pattamadilok, 2012; Pattamadilok, Perre, \& Ziegler, 2011). Thus, whereas the observed crossmodal MMN enhancement may be a neurophysiological marker of initial and automatic letter-speech sound integration and/or representation (Näätänen et al., 2007; Näätänen, 2001), the crossmodal LN enhancement may reflect more elaborate, explicit associative processes that, in children but not in adults, are recruited for the integration of simple letter-speech pairs.

The same crossmodal MMN paradigm (Froyen et al., 2009, 2008, 2011), was used in 11 year-old dyslexic children after 4 years of reading instruction, and indicated reduced integration of letters and speech sounds, with significant letter effects only in the late LN window (Froyen et al., 2011). Further evidence for a reduced sensitivity to letter-speech sound associations in dyslexia comes from fMRI studies showing an underactivation of superior temporal cortex during letter-speech sound integration in 9-year-old dyslexic children (Blau et al., 2010) and adults (Blau et al., 2009), as well as from the absence of a crossmodal MMN enhancement for spoken-written syllable pairs in dyslexic adults (Mittag, Alho, et al., 2013). Similarly, recent studies suggest reduced orthographic-phonological integration in dyslexics on word reading tasks (Hasko, Bruder, Bartling, \& Schulte-Körne, 2012; Hasko, Groth, Bruder, Bartling, \& Schulte-Körne, 2013; Savill \& Thierry, 2011, 2012).

In the current study, we investigate EEG measures of letter-speech sound integration in 9-year-old typical and dyslexic readers and specifically test whether they scale with behavioral measures of reading fluency and readingrelated skills. To this end we employ an adapted version of the audiovisual paradigm of Froyen and colleagues (Froyen et al., 2011) in typical readers $(n=20)$ and age-matched dyslexic children $(n=36)$ with variable levels of reading (dys)fluency. By focusing on 9-year old children, we extend previous ERP findings to a different age group, 2.5 years after reading instruction, intermediate between the previously studied age groups, namely typical readers with 1 or 4 years of reading instruction (Froyen et al., 2009) and dyslexic 
readers with 4 years of reading instruction (Froyen et al., 2011). This age range also coincides with the age of participants from a previous fMRI study reporting reduced neural letter-speech sound integration in dyslexics, as well as correlations between these neural measures and behavioral reading scores (Blau et al., 2010), allowing us to further investigate the same processes with a different technique at the same level of development. As the morphology, amplitude and timing of ERP components are known to be age dependent (Bonte \& Blomert, 2004; Maurer et al., 2006; Oades, Dmittmann-Balcar, \& Zerbin, 1997; Ponton, Eggermont, Kwong, \& Don, 2000; Shafer, Morr, Kreuzer, \& Kurtzberg, 2000), we chose to use a chronologically age matched control group, rather than a reading age matched control group. Finally, including a larger group of dyslexic children with variable levels of reading fluency, allowed us to demonstrate brain-behavior relations not only across typical and dyslexic readers but also to show interindividual differences within the group of dyslexic readers.

\section{Methods}

\subsection{Participants}

In total, 61 children (41 dyslexic and 20 typical readers) participated in the EEG experiment. All children were $3^{\text {rd }}$ graders, native Dutch speakers, having received 2.5 years of reading instruction. Data of 56 children were included in the analysis, including 36 dyslexic readers (9.0 years old, range: 8.2-9.9; 16 girls; 6 left-handed, as assessed by an adapted version of Annett's handedness questionnaire (Annett, 1979), and 20 age-matched typical readers $(8.8$ years old range: 8.3-9.5; 12 girls; 2 left-handed). Data of five children with dyslexia were discarded, 4 children did not complete the EEG measurement and 1 child moved excessively during the EEG measurement.

Dyslexic children were recruited from a specialized institute for dyslexia and reading problems. Prior to the present study, they were diagnosed as dyslexic after an extensive cognitive psycho-diagnostic procedure by the institute. Each of the dyslexic children scored in the lowest 10 percentile of the age appropriate group on standard reading tests (see below). Other behavioral 
scores such as phonological skills or rapid automatized naming (RAN), were not included as selection criteria. Typical readers had reading score percentile of 25 or higher and were recruited from $3^{\text {rd }}$ grades of primary schools in the same area and had similar social-demographic characteristics. All children had a normal hearing and normal or corrected to normal vision, as reported by parents. Comorbidity with behavioral and/or attention disorders was assessed with the Child Behavior Checklist (CBCL) from the Achenbach system of empirically based assessment (ASEBA) (Achenbach \& McConaughy, 2003) which was completed by the parents, and used as an exclusion criterion. Children with below average IQ were also excluded from the experiment. Written informed consent was obtained from the parents of each child. Each child received a small present after the experiment and travel costs were reimbursed. The approval for the research was obtained from a local ethical committee of the Developmental Psychology department of the University of Amsterdam.

\subsection{Behavioral tests}

Prior to the EEG measurements, each of the children performed standard language tests including the word reading, spelling, letter-speech sound identification, letter-speech sound discrimination, rapid automized naming (RAN) and basic reaction time subtests of the 3DM battery (Dyslexia Differential Diagnosis; 3DM, (Blomert \& Vaessen, 2009), as well as a oneminute word reading test (EMT) (Brus \& Voeten, 1973), and reading of a short story 'De kat' ('The cat') (de Vos, 2007). In addition, we used a paper and pencil version of the RAVEN Coloured Progressive Matrices to assess nonverbal IQ scores (RAVEN CPM) (Raven, Raven, \& Court, 1998). We performed a median split of the 36 dyslexic children based on their fluency score to form groups of dysfluent dyslexic readers $(n=18)$ and severely dysfluent dyslexic readers $(n=18)$ (for similar approaches see Faulkner \& Levy, 1999; Fuchs, Fuchs, \& Compton, 2004; Stanovich, Feeman, \& Cunningham, 1983). Because our three individual word reading fluency measures were highly correlated $(r>.919)$, reading fluency was quantified using a composite score of the 3DM word reading tests, the EMT, and "De Kat". Subject characteristics 
and the results of the behavioral tests are shown in Table 2-1. Group differences were tested using one way ANOVAs for each of the pairwise group comparisons and are reported in the Results section.

\subsection{Stimuli}

The stimuli were the same as in Froyen et al. (Froyen et al., 2009, 2008, 2011) including auditory stimuli consisting of the Dutch vowels /a/ and /o/ spoken by a native Dutch female speaker. The vowels were digitally recorded (sampling rate $44.1 \mathrm{kHz}, 16 \mathrm{bit}$ quantization), band-pass filtered $(180-10.000 \mathrm{~Hz})$, resampled at $22.05 \mathrm{kHz}$ and matched for loudness with Praat software (Boersma \& Weenink, 2002). Phoneme duration was $384 \mathrm{~ms}$ for vowel /a/ and $348 \mathrm{~ms}$ for vowel /o/. Vowels were presented binaurally through headphones at a comfortable listening level of $\sim 65 \mathrm{~dB}$ as measured with an analog loudness meter. The visual stimulus was a white, lower case letter "a", presented in size 40 "Arial" font in the center of a computer screen with a black background. The stimuli were presented using Presentation 14.4 (Neurobehavioral Systems, Inc., Albany, CA).

\subsection{Experimental Design}

In one auditory and two audiovisual oddball paradigms, we presented the vowel /a/ as the standard (83\%) and the vowel /o/ as the deviant (17\%) stimulus. In the auditory condition participants listened to the vowels while watching silent movies. In the two audiovisual conditions, the presentation of the standard or deviant vowel was paired with a white letter "a", presented for $500 \mathrm{~ms}$ at the center of a black computer screen. Between the successive letter presentations a white fixation cross appeared in the same location. Both auditory and audiovisual trials had a trial length of $1700 \mathrm{~ms}$. The two audiovisual conditions differed in the stimulus onset asynchrony (SOA) between letter and vowel presentation. In the first audiovisual condition (AV0), the letter and vowel appeared simultaneously, and in the second audiovisual condition (AV200), the letter appeared $200 \mathrm{~ms}$ before the vowel. During the audiovisual conditions, participants performed a simple visual target detection task to ensure fixation on the screen. The visual target consisted of a color picture of a wrapped 
present, which was presented instead of the letter in 3,5\% of the trials (10 trials per block) and required a button press. This paradigm was the same as the one used in Froyen et al., (Froyen et al., 2009, 2008, 2011) except for the following two changes. First, the trial length was increased from $1250 \mathrm{~ms}$ to $1700 \mathrm{~ms}$ because the experiment was part of a larger study involving coherence analysis at lower frequencies. Second, in order to obtain sufficient deviant trials within 76 minutes of measurement time, the number of deviants was increased from $10 \%$ to $17 \%$, a percentage that was previously shown to lead to reliable mismatch responses (Bonte, Mitterer, Zellagui, Poelmans, \& Blomert, 2005; Bonte et al., 2007). Participants performed three blocks of each condition, each block consisting of 288 trials. Per condition a total of 150 deviant and 714 standard trials were presented. Standards and deviants were presented in pseudo-random order: two successive deviants were required to be separated by at least three standards, and deviants could not be one of the first two trials of a block. The blocks of the same condition were presented in consecutive fashion, the order of the conditions was pseudorandomized and counterbalanced, with one of the cross-modal conditions always being presented first.

\subsection{EEG data recording and analysis}

EEG data were recorded at a $1024 \mathrm{~Hz}$ sampling rate and a DC-104 Hz recording bandwidth using the Biosemi Active Two system (Biosemi, Amsterdam, Netherlands). EEG was measured from 64 active-channels, placed according to the 10-20 international system (Electro-cap International Inc., with CMS and DRL electrodes at the approximate locations of PO1 and PO2), and the CMS was used as a recording reference. Eye-movements were measured using 4 additional Flat-Type Active electrodes, 2 were placed below and above left eye and 2 at the outer canthi of each eye. In addition 2 electrodes were placed on the right and left mastoids and used for offline re-referencing. The offset range of the electrodes was kept between $-20 \mathrm{mV}$ and $20 \mathrm{mV}$.

For the analysis we discarded the first 2 trials of each block, as well as the first epoch after each deviant and, in the audiovisual conditions, the first 2 epochs after catch trials to account for possible movement artifacts due to button 
presses (Luck, 2005). Per participant, we included data of the 150 deviant trials and of 150 standard trials chosen randomly from the pool of 480 standard trials. Data were analyzed using the v11.0.0b EEGLAB toolbox (Delorme \& Makeig, 2004)( http://www.sccn.ucsd.edu/eeglab) and custom Matlab scripts (MATLAB 2011b, The MathWorks, Natick, MA). EEG data pre-processing included offline rereferencing to the average of the left and right mastoids, applying a bandpass filter of $1-70 \mathrm{~Hz}$ and downsampling to $256 \mathrm{~Hz}$. The EEG data was epoched from $-500 \mathrm{~ms}$ to $1200 \mathrm{~ms}$ with respect to the auditory (Auditory and AV0 condition) or visual (AV200) stimulus and baseline corrected with respect to the mean signal in the $500 \mathrm{~ms}$ baseline period. Removal of artifacts was performed in two steps. First, the data were visually inspected and epochs containing non-stereotypical artifacts including high amplitude, high-frequency muscle noise, swallowing and electrode cable movements, were rejected. Secondly, stereotypical artifacts, including eye movements, eye blinks and heart beat artifacts were corrected with extended INFOMAX ICA (Lee, Girolami, \& Sejnowski, 1999) as implemented in EEGLAB. ICA was performed on 64 scalp channels resulting in 64 components per condition per participant which were classified as EEG or artificial activity based on scalp topography, time course and power-frequency spectra (Jung et al., 2000). Single electrodes containing high amplitude noise were interpolated using spherical interpolation after the data was reconstructed. Component activations representing non-brain artifacts were removed, and EEG data were reconstructed from the remaining component activations representing brain activity. In typical readers, the reconstructed data was based on a mean (SD) number of 41 (4) components per subject in the auditory condition, 47 (4) in the Av0 condition and 44 (5) in the Av200 condition. The number of EEG components in the two subgroups of dyslexic children corresponded to - dysfluent dyslexics: 43 (4) components per subject in the auditory condition, 48 (4) in the Av0 condition and 46 (4) in the Av200 condition; severely dysfluent dyslexics: 42 (8) components per subject in the auditory condition, 45 (7) in the Av0 condition and 44 (7) in the Av200 condition. The reconstructed data was baseline corrected and low pass filtered at $30 \mathrm{~Hz}$. 


\section{Reading Fluency and Cross-Modal ERPs in Dyslexia}

Event related potentials (ERPs) were obtained by averaging the epochs per stimulus per condition for each participant separately. In typical readers, the mean (SD) number of epochs included in the averages for the standards and deviants corresponded to 149 (1) and 149 (1) in the auditory condition, 148 (2) and 149 (2) in the AV0 condition and 148 (2) and 149 (1) in the AV200 condition. In dysfluent dyslexics these values were 149 (1) and 148 (2) in the auditory condition, 148 (2) and 148 (2) in the AV0 condition and 148 (2) and 148 (2) in the AV200 condition. Lastly, in severely dysfluent these values were 148 (5) and 148 (5) in the auditory condition, 148 (2) and 148 (2) in the AV0 condition and 148 (2) and 147 (3) in the AV200 condition.

We evaluated the presence of the mismatch negativity (MMN) and late negativity (LN) by analyzing ERPs evoked by standard and deviant stimuli in the auditory and both audiovisual conditions, in each of our subject groups. As maximal cross-modal effects were expected across fronto-central electrodes (Froyen et al., 2009, 2011), analysis was performed on four fronto-central sites (Fz, Cz, FC3, FC4). The timing of the MMN and $\mathrm{LN}$ responses was measured by determining individual peak latencies within their respective time windows: (1) 100 to $250 \mathrm{~ms}$ for the MMN and (2) $600 \mathrm{~ms}-750 \mathrm{~ms}$ for the LN (Froyen et al., 2009, 2011). The amplitude of the MMN and LN responses was measured by determining the mean amplitude in the $50 \mathrm{~ms}$ around the individual peak latencies. We first applied mixed model ANOVAs with stimulus (2 levels: standard and deviant), condition (3 levels: Au, Av0, Av200) and electrode (4 levels) as within subjects factors and group (3 levels: typical, dysfluent dyslexic and severely dysfluent dyslexic readers) as between subjects factor. Because MMN and LN responses were detected in all conditions and all subject groups, we subsequently performed our analyses of crossmodal letter effects on the difference waves obtained by subtracting the ERPs evoked by standards from those of deviants. MMN/LN amplitude and latency measures were analyzed using mixed model ANOVAs on pairs of conditions (Au-Av0; Au-Av200) and electrode (4 levels: Fz, Cz, FC3, FC4) as within subject factors. We report Greenhouse-Geisser corrected p-values. In a further analysis, we separately analyzed the N1 and P2 responses to standard and deviant stimuli to investigate 
the relation between the crossmodal MMN effects and these event-related responses. N1 and P2 amplitudes were statistically assessed using repeated measures ANOVAs with Stimulus (2 levels: standard, deviant) and Electrode (4 levels: $\mathrm{Fz}, \mathrm{Cz}, \mathrm{FC} 3, \mathrm{FC} 4)$ as within subject factors.

We used linear regression to investigate the relation between individual differences in ERP correlates of letter-sound associations and behavioral measures of reading fluency and related skills. ERP measures consisted of (1) MMN latency in the two audiovisual (AV0 and AV200) conditions, and (2) the MMN and LN letter-effect in the two audiovisual conditions (i.e. the MMN/LN amplitude enhancement in the audiovisual as compared to auditory conditions, Froyen et al., 2009, 2008, 2011). For linear regression, ERP measures were quantified using composite scores across 4 fronto-central electrodes (Fz, Cz, FC3 and FC4). Our selection of behavioral measures of interest was based on (1) our research aim, i.e. to test the relation between reading fluency and cross-modal ERP measures, and (2) results of a large-scale study in Dutch, Hungarian and Portuguese school children in which factor analysis of test scores on 3DM subtests identified verbal working memory, phonemic awareness, RAN, letter-speech sound accuracy and letter-speech sound speed as separate factors (Vaessen et al., 2010). Thus, reading fluency, was quantified using a composite score of the 3DM word reading tests, the EMT, and "De Kat". Furthermore, the other behavioral measures included reading accuracy $(3 \mathrm{DM})$, letter-speech sound coupling accuracy (spelling, letterspeech sound identification and discrimination, $\mathrm{r}>$.406) and speed (spelling, letter-speech sound identification and discrimination, $\mathrm{r}>.391$ ), phonological awareness (3DM phoneme deletion accuracy) and rapid auditory naming (3DM RAN total score). We employed simple linear regressions with one of the ERP measures as a predictor and one of the behavioral composite scores as a dependent variable to probe relations of each ERP measure specifically to the behavior. As we conducted several linear regressions, i.e. 12 for latencies and 24 for amplitudes, once for all the children and once only for dyslexics, we accounted for multiple comparisons by using FDR corrections (Benjamini \& Hochberg, 1995) and report p values relative to obtained q(FDR) thresholds. 
Reading Fluency and Cross-Modal ERPs in Dyslexia

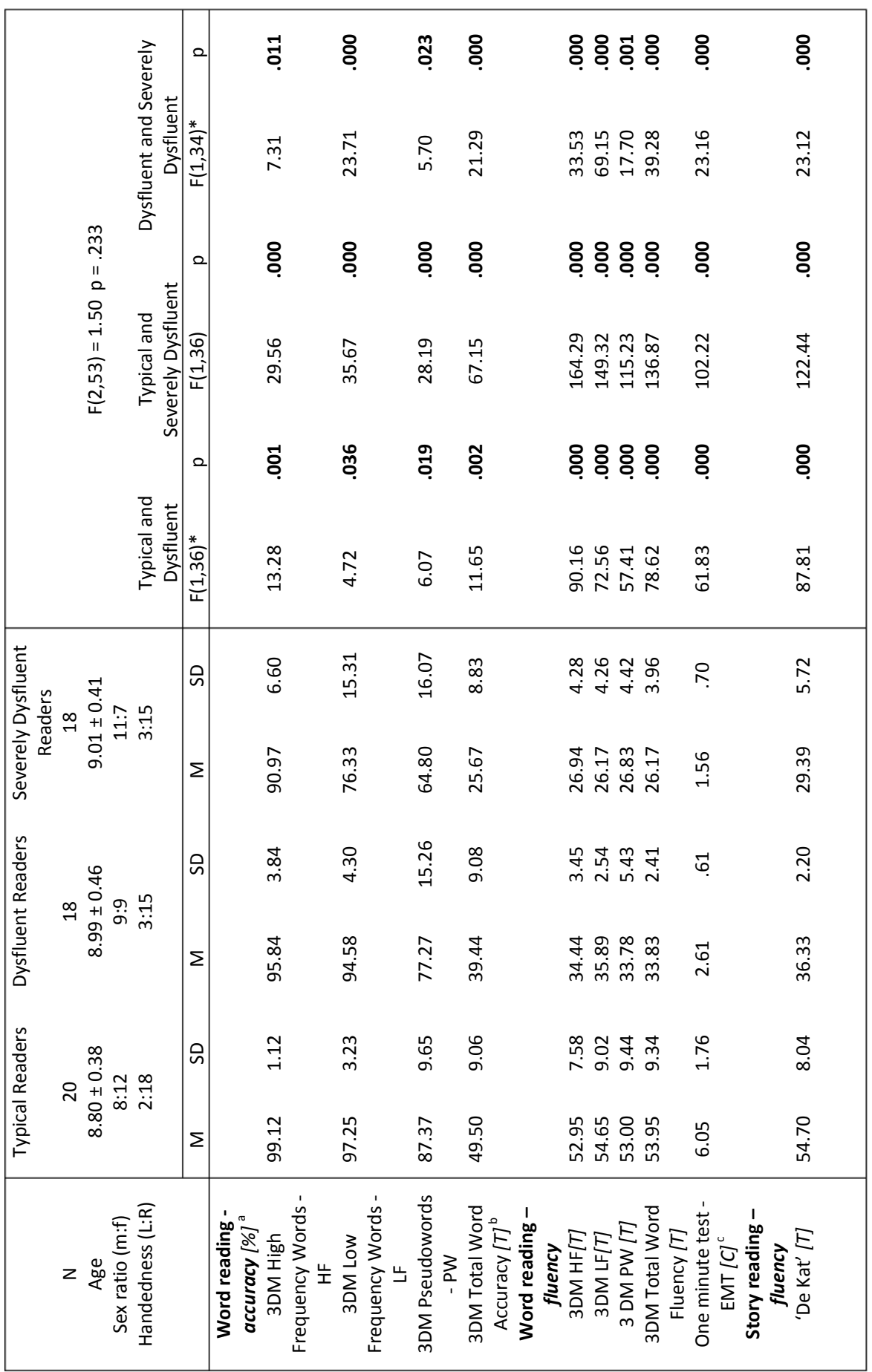




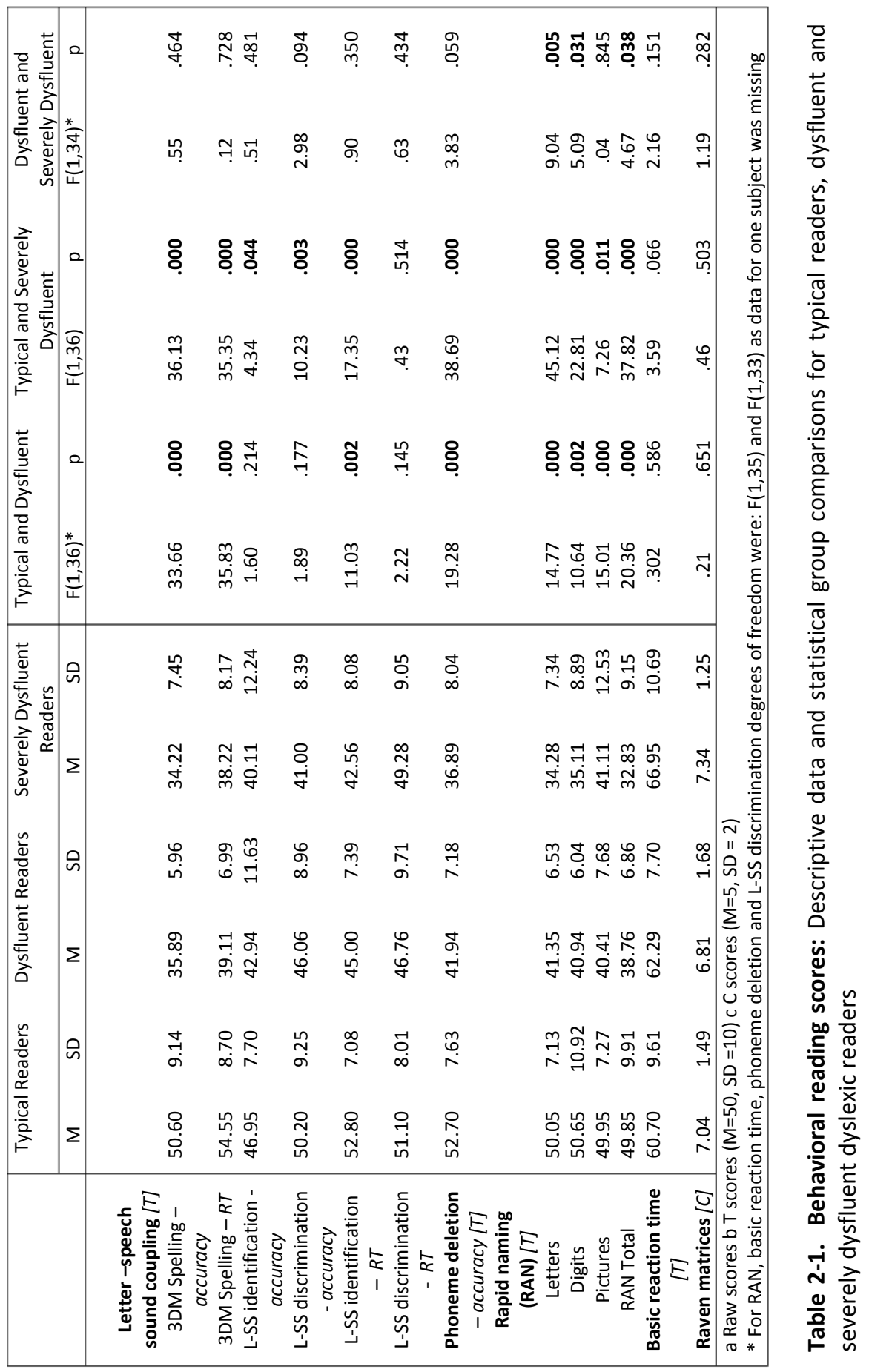




\section{Results}

\subsection{Behavioral tests: Typical readers, Dysfluent and Severely Dysfluent Dyslexic readers}

The dyslexic readers were divided into a dysfluent and a severely dysfluent group, using a median split based on their reading fluency scores (see Methods). A comparison of behavioral test scores showed significantly lower scores for severely dysfluent as compared to dysfluent dyslexics on all word reading tests (fluency as well as accuracy), as well as on rapid automatized naming (Table 21). The dyslexic subgroups did not significantly differ on any of the other behavioral tests. Both dyslexic groups scored significantly lower than typical readers on all language tests with the exception of the accuracy of letter-speech sound discrimination and identification (comparable in dysfluent and typical readers) and the speed of letter-speech sound discrimination (comparable in all three groups). Non-verbal IQ and speed of processing (reaction times) were comparable across the three groups.

\subsection{Auditory MMN and LN effects}

Because these measures served as a baseline for assessing cross-modal enhancement effects, we first analyzed the MMN and LN responses evoked in the auditory condition. In each of the three subject groups, the deviant elicited a comparable auditory $\mathrm{MMN}$, with the expected fronto-central topographical distribution (Figures 2-1 and 2-2). Comparable MMN responses lead to a main effect of Stimulus $\left(\mathrm{F}(1,53)=105.26, \mathrm{p}=.000, \eta^{2}=.665\right)$, without significant group differences. We also did not observe significant group differences in the latency of the auditory MMN.

In each group, the vowel deviant additionally elicited a weaker and more sustained auditory LN response around 670-690 ms after stimulus onset (Figures 2-1 and 2-2). Statistical analyses indicated a comparable auditory LN response across groups, with a significant main effect of Stimulus $(F(1,53)=$ 64.44, $\left.\mathrm{p}=.000, \eta^{2}=.549\right)$ and no significant group differences for amplitudes or latencies. In sum, in each of the three groups the deviant vowel sound /o/ 
elicited comparable MMN and LN responses with respect to the standard vowel sound $/ \mathrm{a} /$.

\subsection{Letter-effects: $M M N$ and $L N$ amplitude enhancements}

In both cross-modal conditions (Av0 and Av200) the deviant vowel sound /o/ elicited MMN and LN responses with respect to the standard vowel sound /a/ (Fig. 2-1 and Fig. 2-2). In these blocks, the letter "a" was presented together with the standard and deviant vowels, leading to a double violation: the deviant speech sound /o/ violated the expectation built by both the standard speech sound / a/ and the letter ' $a$ '. To assess the neural integration of letters and speech sounds we tested whether this double violation significantly enhanced the cross-modal as compared to the auditory MMN and LN responses (Froyen et al., 2009, 2008, 2011). To this end, we first performed a mixed model ANOVA with stimulus (standard, deviant), condition (auditory, AV0, AV200) and electrodes (4 fronto-central electrodes) as within-subject factors and subject group as between-subject factor. In the MMN window, this overall analysis indicated stronger responses to deviants as compared to standards (Stimulus $\left.\mathrm{F}(1,53)=294.61, \mathrm{p}=.000, \eta^{2}=.848\right)$, and stronger cross-modal as compared to auditory (mismatch) responses (Condition $\mathrm{F}(2,99)=50.61, \mathrm{p}$ $=.000, \eta^{2}=.488$; Stimulus*Condition: $\left.\mathrm{F}(2,105)=12.29, \mathrm{p}=.000, \eta^{2}=.188\right)$, as well as a tendency for groups to differ in their response amplitudes across conditions (Condition*Group $\left(\mathrm{F}(4,99)=2.18, \mathrm{p}=.081, \eta^{2}=.076\right)$. Pairwise group comparisons showed that this tendency was driven by significant differences between severely dysfluent dyslexics and typical readers (Condition*Group: $\left.\mathrm{F}(2,69)=3.87, \mathrm{p}=.027, \eta^{2}=.097\right)$, while there were no differences in other cases.

Also in the later LN window, the overall mixed-model ANOVA indicated stronger responses to deviants as compared to standards (Stimulus $\left.\mathrm{F}(1,53)=267.28, \mathrm{p}=.000, \eta^{2}=.835\right)$ and stronger cross-modal as compared to auditory (mismatch) responses (Condition $\mathrm{F}(1,73)=9.47, \mathrm{p}=.001, \eta^{2}=.152$; Stimulus*Condition: $\left.\mathrm{F}(2,99)=8.78, \mathrm{p}=.000, \eta^{2}=.142\right)$ together with significant group differences in response amplitudes across conditions (Condition*Group: $\left.\mathrm{F}(3,73)=3.87, \mathrm{p}=.015, \eta^{2}=.127\right)$. Pairwise group comparisons showed that the 
significant group difference in response amplitudes across conditions was driven by significant differences between severely dysfluent dyslexics and typical readers (Condition*Group: $\mathrm{F}(1,51)=4.49, \mathrm{p}=.027, \eta^{2}=.111$ ) as well as between dysfluent dyslexics and typical readers (Condition*Group: $\left.\mathrm{F}(1,49)=6.80, \mathrm{p}=.006, \eta^{2}=.159\right)$, but not by a difference between the two dyslexic groups $\left(\mathrm{F}(1,46)=0.48, \mathrm{p}=.549, \eta^{2}=.014\right)$.
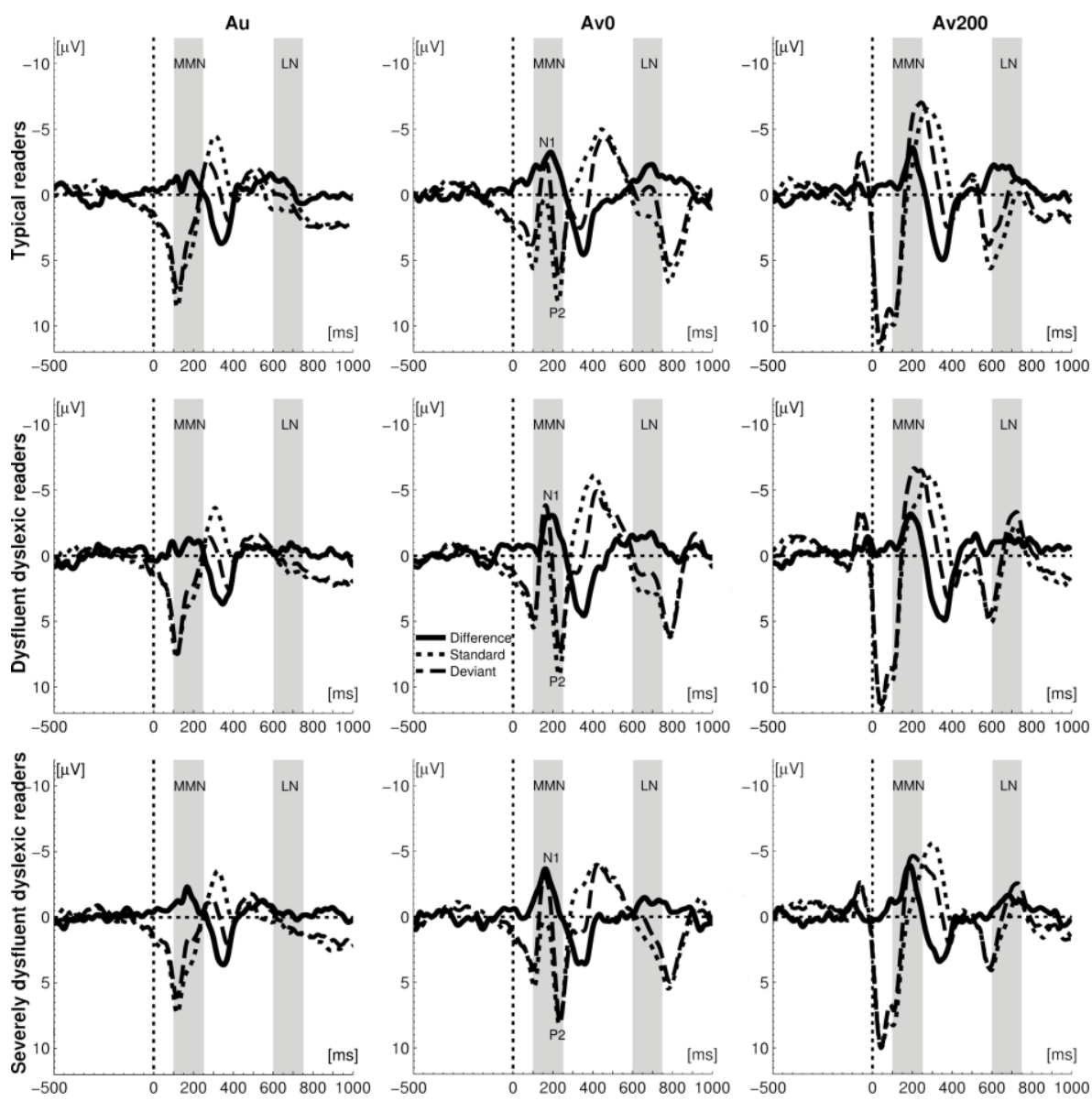

Figure 2-1. Grand average event-related potentials (ERP) and difference waves: Grand average ERPs averaged over 4 frontocentral electrodes for standard (dotted line), deviant (dashed line) and their difference (solid line) in auditory ( $\mathrm{Au}$ ) and two audiovisual conditions (Av0 and Av200) with time intervals of interest shaded in light grey. N1 and P2 peaks of standard and deviant ERPs are also marked in synchronous audiovisual condition (Av0). 


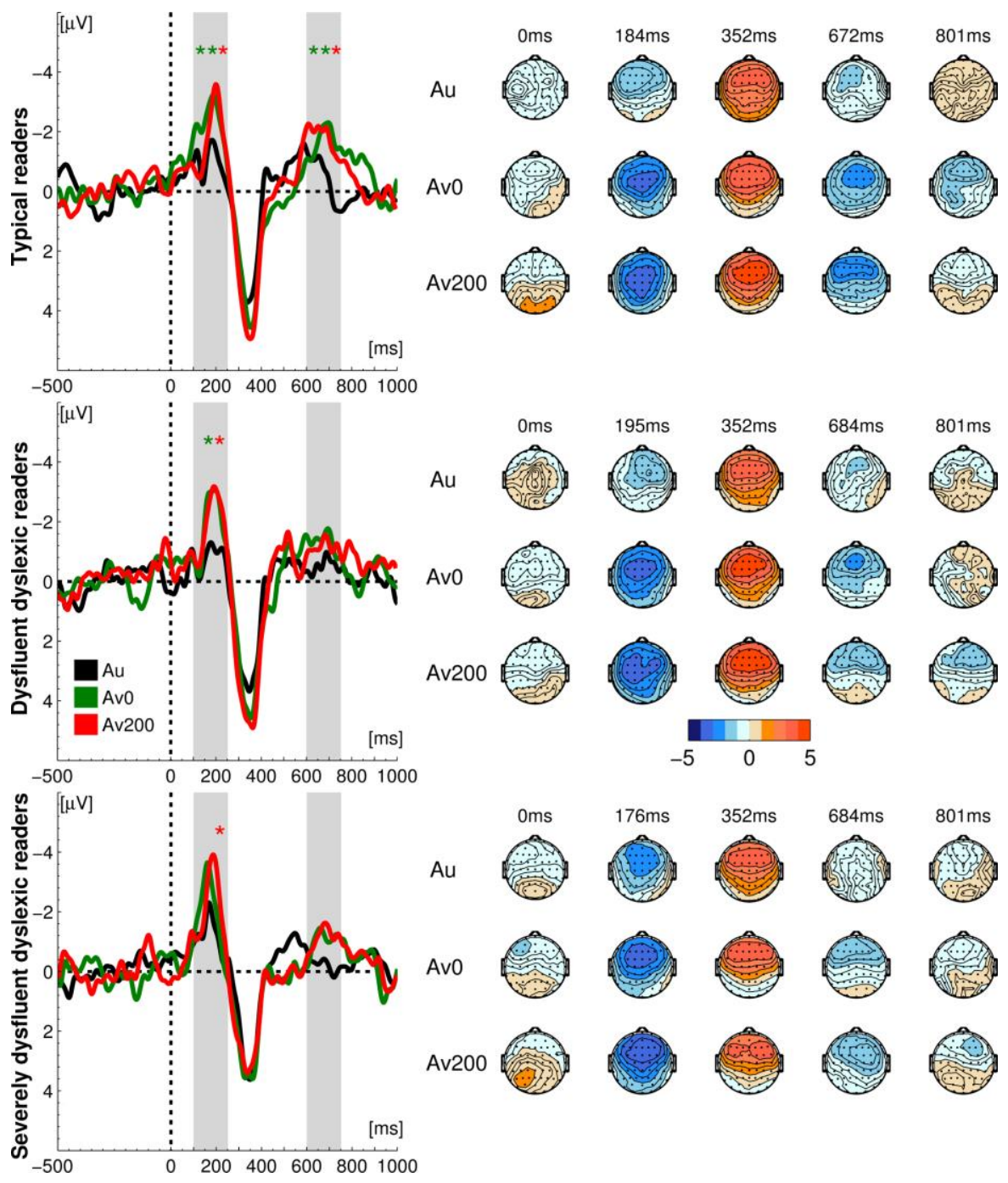

Figure 2-2. Difference waves and topographical maps: I. Difference waves (averaged over 4 frontocentral electrodes) with time intervals of interest shaded in gray. Significant differences between conditions are marked with asterisks $\left({ }^{*} p<=.05 ;{ }^{*} p<=.01\right)$ : green asterisk - Av0 vs. Au difference, red asterisk - Av200 vs. Au difference. II. Topographical distribution of average amplitudes in difference waves over 64 scalp electrodes

We further analyzed these condition and group effects by performing mixed model ANOVAs on the MMN difference waves with Condition (AV0 versus 


\section{Reading Fluency and Cross-Modal ERPs in Dyslexia}

Auditory; AV200 versus Auditory) and electrodes (4 fronto-central electrodes) as within subjects factors. In the simultaneous cross-modal condition (AV0), significant MMN amplitude enhancements were observed in typical readers $\left(\mathrm{F}(1,19)=10.92, \mathrm{p}=.004, \eta^{2}=.365\right)$ as well as in dysfluent dyslexics $(\mathrm{F}(1,17)$ $\left.=7.63, \mathrm{p}=.013, \eta^{2}=.310\right)$, whereas in severely dysfluent dyslexics this enhancement was not significant $\left(F(1,17)=3.43, p=.082, \eta^{2}=.168\right)$. When letter presentation preceded speech sound onset (AV200) significant cross-modal MMN enhancements were found in all three subject groups (typical readers: $\mathrm{F}(1,19)=7.21, \mathrm{p}=.015, \eta^{2}=.275$; dysfluent dyslexics: $\mathrm{F}(1,17)=5.93, \mathrm{p}=.026$, $\eta^{2}=.259$; severely dysfluent dyslexics: $\left.\mathrm{F}(1,17)=6.09, \mathrm{p}=.024, \eta^{2}=.264\right)$.

As for the $\mathrm{LN}$ window, in typical readers the presence of a letter significantly enhanced the LN amplitude in both cross-modal conditions (AV0 vs. Auditory: $\mathrm{F}(1,19)=10.90, \mathrm{p}=.004, \eta^{2}=.365$; AV200 vs. Auditory: $\mathrm{F}(1,19)=7.85, \mathrm{p}=.011$, $\left.\eta^{2}=.292\right)$. In contrast, in the dyslexic groups the LN letter effects only resulted in non-significant trends (AV0 vs. Auditory, dysfluent: $\mathrm{F}(1,17)=3.93, \mathrm{p}=.064$, $\eta^{2}=.188$; severely dysfluent: $\mathrm{F}(1,17)=3.98, \mathrm{p}=.062, \eta^{2}=.190 ;$ Av200 vs. Auditory, dysfluent: $\left(\mathrm{F}(1,17)=3.44, \mathrm{p}=.081, \eta^{2}=.168\right.$; severely dysfluent: $\left.\mathrm{F}(1,17)=2.41, \mathrm{p}=.139, \eta^{2}=.124\right)$.

In summary, in both cross-modal conditions, our results showed early (MMN) and late (LN) letter effects in typical readers. Although the group differences in MMN and LN letter effects were subtle, the dysfluent group resembled typical readers in the presence of an MMN letter effect, whereas they resembled the severely dysfluent group in the lack of a significant LN letter effect. The severely dysfluent group differed most from typical readers in both the lack of a significant LN letter effect and in only showing a significant MMN letter effect in the AV200 condition.

\subsection{Letter effects: $M M N$ and LN latency}

We investigated the timing of the letter effects, by analyzing MMN and LN latencies in both crossmodal conditions. As already indicated by our regression analysis, groups tended to differ in MMN response latency in the simultaneous 
crossmodal (AV0) condition (Group $\left(\mathrm{F}(2,53)=5.85, \mathrm{p}=.062, \eta^{2}=.181\right.$ ). In particular, severely dysfluent dyslexics showed a significantly shorter MMN latency than both the typical readers $\left(F(1,36)=8.59, \mathrm{p}=.006, \eta^{2}=.193\right)$, and the dysfluent dyslexics $\left(\mathrm{F}(1,34)=7.65, \mathrm{p}=.009, \eta^{2}=.184\right)$, whereas the latter groups showed similar latencies $\left(F(1,36)=0.07, p=.787, \eta^{2}=.002\right)$. No significant group differences were found for MMN latency in the asynchronous cross-modal condition (AV200), or for LN latency in either of the cross-modal conditions.

\subsection{ERP - behavior relations}

We used linear regression to investigate the relation between individual differences in MMN/ LN responses in the crossmodal conditions and behavioral measures of reading fluency and related skills. Results showed that such a relation was present for the latency of the MMN response elicited in the simultaneous crossmodal condition (AV0). First, across both typical and dyslexic readers, MMN latency in the AV0 condition significantly correlated (significance threshold $\mathrm{q}(\mathrm{FDR})=.010)$ with individual differences in word reading fluency $(\mathrm{r}=.368 ; \mathrm{p}=.005$; Table $2-2$, Figure 2-3), word reading accuracy $(\mathrm{r}=.398 ; \mathrm{p}=.002)$ and phoneme deletion accuracy $(\mathrm{r}=.343 ; \mathrm{p}=.001)$. When restricting the analysis to the dyslexic readers, a significant relation was only shown for reading fluency $(\mathrm{r}=.554 ; \mathrm{p}=.000)$. Remarkably, in both cases, longer MMN peak latencies were found in the more fluent readers, a result that is further investigated in our analysis of the N1-P2 responses underlying the MMN effect. None of the other ERP-behavior regressions reached statistical significance. 


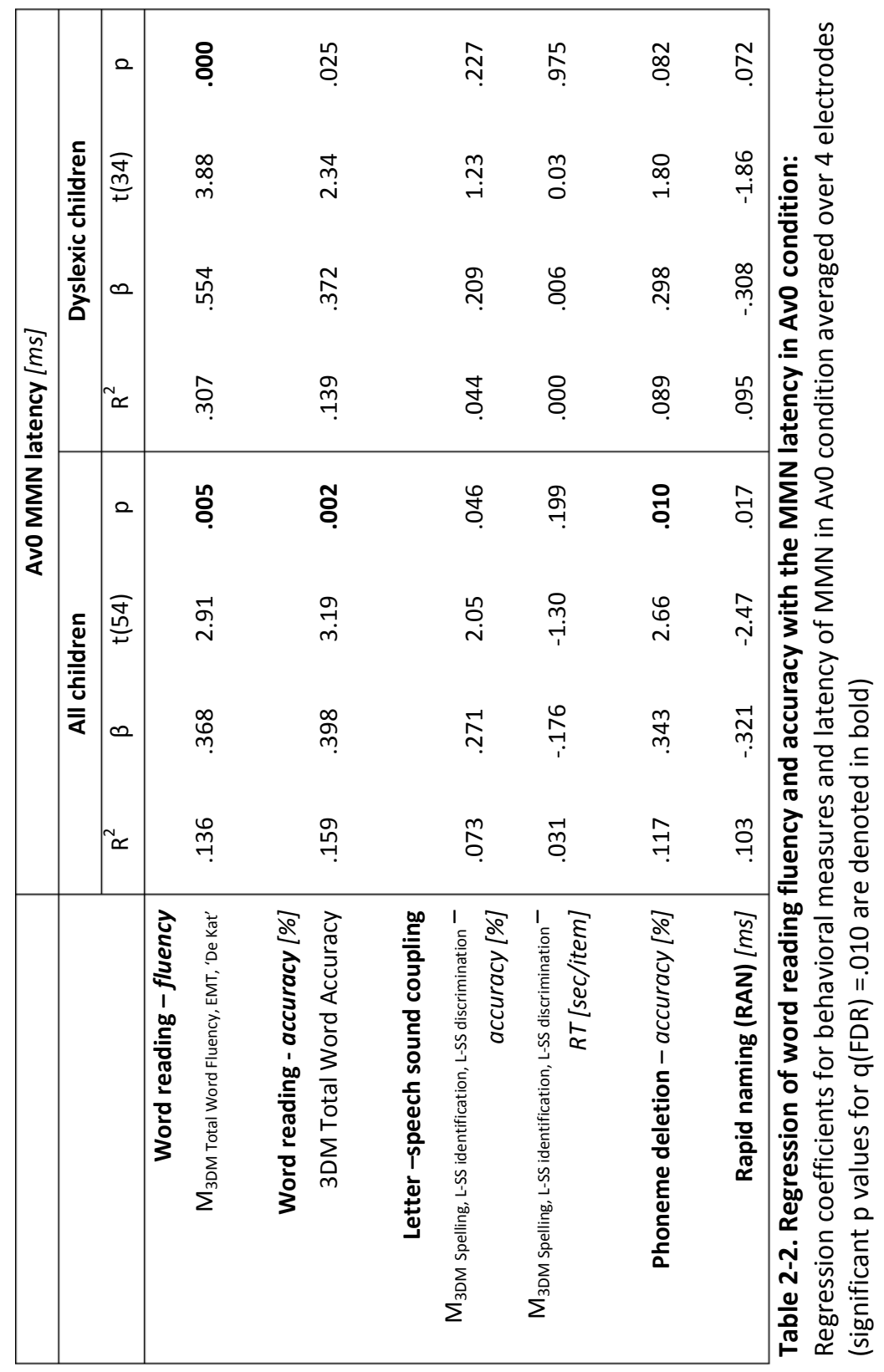



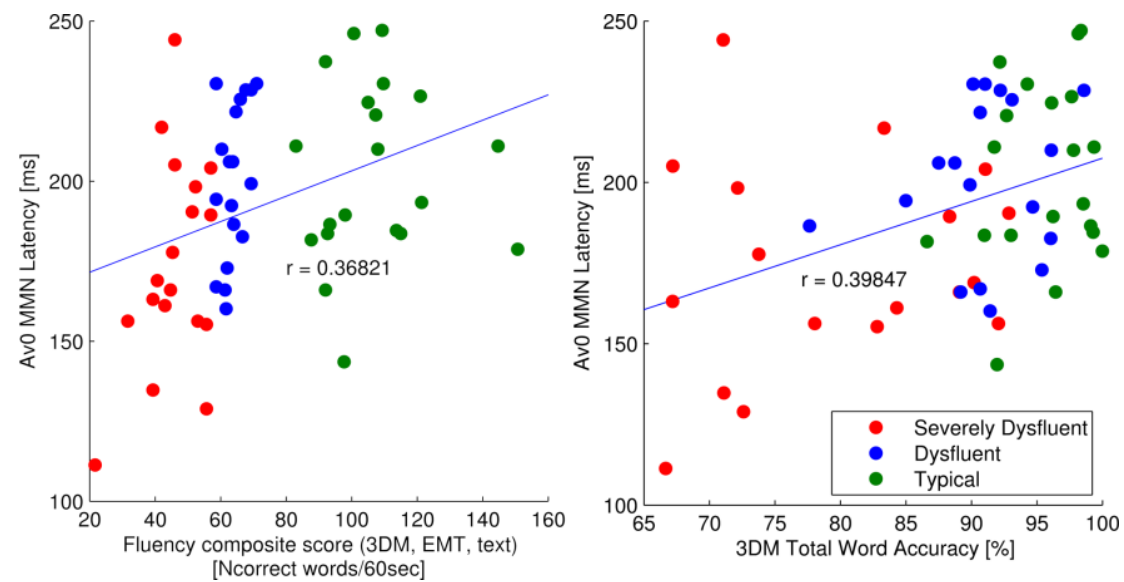

Figure 2-3. Correlations of word reading fluency and accuracy with the MMN latency in Av0 condition: Relation of composite fluency (3DM word reading fluency, EMT, 'De Kat') and 3DM Accuracy (HFW, LFW, PW) raw scores with MMN latency in $\mathrm{AvO}$ condition with the strength of correlation represented by $r$.

\subsection{Relation between MMN and N1-P2 responses}

In a final analysis we aimed to further investigate the origin of the shorter MMN latency in severely dysfluent dyslexics as compared to both other groups, as well as the corresponding regression results in the simultaneous cross-modal (AV0) condition (Figure 2-3) by analyzing the timing of the MMN with respect to the evoked N1 and P2 responses (Figure 2-1). Results showed that in severely dysfluent dyslexics, letter-speech sound pairs only elicited a deviancy effect in the $\mathrm{N} 1$ window (main effect of Stimulus $\mathrm{F}(1,17)=28.32, \mathrm{p}=.000$, $\left.\eta^{2}=.625\right)$, but not in the P2 window $\left(\mathrm{F}(1,17)=.03, \mathrm{p}=.860, \eta^{2}=.002\right)$, as can also be seen in Figure 2-1. In contrast, typical readers as well as dysfluent dyslexics showed a deviancy effect in both the N1 and the P2 windows (main effects of Stimulus typical readers $\mathrm{N} 1: \mathrm{F}(1,19)=32.27, \mathrm{p}=.000, \eta^{2}=.629 ; \mathrm{P} 2: \mathrm{F}(1,19)$ $\left.=6.49, \mathrm{p}=.020, \eta^{2}=.255\right)$; dysfluent dyslexics N1: $\mathrm{F}(1,17)=14.24, \mathrm{p}=.002$, $\eta^{2}=.456$ and P2: $\left.F(1,17)=10.15, p=.005, \eta^{2}=.374\right)$. The absence of a P2 deviancy effect in the severely dysfluent dyslexics thus most likely explains their shorter MMN latency. 


\begin{tabular}{l|ccc} 
& $\begin{array}{c}\text { Severely dysfluent } \\
\text { dyslexic }\end{array}$ & $\begin{array}{c}\text { Dysfluent } \\
\text { dyslexic }\end{array}$ & Typical \\
\hline MMN & No & Yes & Yes \\
Av0 vs. Au & Yes & Yes & Yes \\
Av200 vs. Au & & & \\
LN & No & No & Yes \\
Av0 vs. Au & No & No & Yes \\
Av200 vs. Au & & & \\
Av0 & Yes & Yes & Yes \\
N1 difference & No & Yes & Yes \\
P2 difference & & &
\end{tabular}

Table 2-3. Summary of results

\section{Discussion}

The present study investigated ERP measures of letter-speech sound integration in typically reading and dyslexic children after 2,5 years of reading instruction and specifically investigated how these ERP measures relate to individual differences in reading fluency and related skills. For this purpose we employed a passive oddball paradigm and tested cross-modal enhancement of the MMN and LN due to an audiovisual violation (vowel sound /o/ vs. vowel sound $/ \mathrm{a} /$ and letter ' $\mathrm{a}$ ') as compared to an auditory only violation (vowel sound /o/ vs. vowel sound /a/; Froyen et al., 2009). Our results show robust neural integration of letters and speech sounds in the typical readers, albeit at a different temporal integration window compared to adults (Froyen et al., 2008). Furthermore, they confirm and extend previous findings in dyslexic children by demonstrating reduced letter-speech sound integration, with different patterns of integration deficiency depending on the level of reading dysfluency (Table 23). 


\subsection{Typical readers: enhanced (and non-selective) neural sensitivity?}

Typical readers after 2,5 years of reading instruction showed cross-modal enhancements of the MMN and $\mathrm{LN}$ responses independent of the precise timing of letter and speech sound presentation (AV0 and Av200), indicating a broad temporal window of integration. This contrasts with previous results of adults, who only showed a cross-modal enhancement in the MMN window, and only when letters and speech sounds were presented simultaneously (Froyen et al., 2008). Furthermore, the present results showed a different pattern of cross-modal effects as compared to previous findings in both typical beginning readers after 1 -year of reading instruction and advanced typical readers after 4-years of reading instruction (Froyen et al., 2009). That is, in the younger readers cross-modal enhancement only reached significance in the LN window and only when the letter preceded the speech sound (AV200). In the advanced readers this pattern shifted with significant cross-modal enhancement in the earlier MMN window when the letter preceded the speech sound (AV200) and additionally in the LN window when letters and speech sounds were presented simultaneously (AV0). In comparison to the younger readers, the observed emergence of a cross-modal MMN enhancement may reflect the initial development of an early detection and integration of letter-sound pairs in 9-year-old children (Näätänen et al., 2007; Näätänen, 2001). In comparison to the more advanced readers, who only showed a cross-modal LN enhancement in the simultaneous AV0 condition, the unspecific cross-modal LN enhancement independent of the timing of letter presentation, may suggest immature and non-selective further processing of letter-sound congruency in 9year-olds. On a more general level, the pattern of stronger and/or less selective cross-modal effects in our intermediate group of typical readers is consistent with a developing neural system for audiovisual speech and letter-sound processing that undergoes dynamic changes throughout primary school years (Blomert, 2011; Knowland, Mercure, Karmiloff-Smith, Dick, \& Thomas, 2014; Nath, Fava, \& Beauchamp, 2011). In particular, they may reflect enhanced neural sensitivity for letter-sound congruency after 2.5 years of reading instruction, before developing into a more selectively tuned system with 
advanced reading practice. Similar nonlinear patterns of an initial sensitivity increase during early school years, followed by reduced and more selective sensitivity in adults, have been observed for print selective visual N1 responses (Maurer et al., 2006) and ERP phonological priming effects during an auditory lexical decision task (Bonte \& Blomert, 2004). On the other hand, also methodological differences between the present study and the previous ERP studies by Froyen and colleagues (Froyen et al., 2009, 2008, 2011) may have (at least partly) contributed to the relatively strong cross-modal ERP effects in our typical readers. For example, unlike these previous studies, we used active electrodes for EEG data acquisition and ICA-based EEG preprocessing, which both lead to improvement of ERP signal-to-noise ratio. Another factor that may have increased signal-to-noise ratio is an increase in trial length from 1250 ms to $1700 \mathrm{~ms}$. In addition, we employed a full within-subjects design as compared to a partial within-subjects design (Froyen et al., 2009). To further understand the development of brain mechanisms for letter-speech sound integration and their relation to reading acquisition, it is important to apply similar cross-modal neuroimaging paradigms in a longitudinal set-up and follow the same children while they are learning to read.

\subsection{Dyslexic children: crossmodal MMN effect scales with reading dysfluency}

ERP data of dyslexic children showed normal auditory MMN and LN responses together with reduced effects of letter-speech sound congruency. Most interestingly results indicated different patterns of integration deficiency depending on the level of reading fluency. In particular, in our regression analysis behavioral measures of reading fluency significantly predicted the latency of the MMN response in the simultaneous cross-modal condition (AV0), not only across typical and dyslexic readers, but also within the group of dyslexic readers. Further analyses indicated that this relation could be explained by a short-lasting, reduced $\mathrm{MMN}$ response, encompassing only the $\mathrm{N} 1$ window in severely dysfluent dyslexics as compared to a longer lasting MMN response, encompassing both the N1 and P2 windows in dysfluent dyslexic and typical readers. In addition, in the AVO condition, MMN latency correlated 
significantly with reading accuracy and phonological awareness (phoneme deletion) across typical readers and dyslexics, but not within the dyslexic group alone.

Although relatively little is known about the functional relevance of the P2, recent evidence suggests that this response is specifically sensitive to the audiovisual integration of orthographic and phonological units (Pattamadilok et al., 2011) as well as of visual articulatory gestures and speech (Baart, Stekelenburg, \& Vroomen, 2014; Knowland et al., 2014; Pilling, 2009; van Wassenhove, Grant, \& Poeppel, 2005). For example, in an elegant study by Baart et al. (Baart et al., 2014), audiovisual integration of articulatory gestures (lipread speech) and sine-wave speech was compared between participants trained to perceive the sine-wave speech in either a 'speech' or 'nonspeech' mode. Whereas lipread speech modulated the N1 response in both processing modes, the P2 was only modulated in listeners who recognized the sine-wave speech as speech, thus suggesting more general audiovisual convergence in the N1 window followed by speech specific audiovisual integration in the P2 window. This speech specificity of the P2 response would also concur with its putative neural source in the posterior superior temporal sulcus (Liebenthal et al., 2010), a region involved in the integration of audiovisual speech (Beauchamp, Lee, Argall, \& Martin, 2004) and of letters and speech sounds (van Atteveldt, Blau, Blomert, \& Goebel, 2010; van Atteveldt et al., 2004). Moreover activity in this region has been found to scale with interindividual differences in audiovisual speech perception (McGurk effect) in typically reading children and adults (Nath \& Beauchamp, 2012; Nath et al., 2011) and to show reduced letter-speech sound integration in dyslexic children (Blau et al., 2010). By analogy, we would speculate that the N1 deviancy effect shown in the simultaneous audiovisual (AV0) condition indicates audiovisual convergence common across the three children groups, followed by a speech specific integration of letters and speech sounds in the P2 window in typical readers and dysfluent dyslexics, which is reduced or absent in severely dysfluent dyslexics. Recently, a diminished speech-specificity of audiovisual integration was also 


\section{Reading Fluency and Cross-Modal ERPs in Dyslexia}

found for spoken-written syllable pairs in Finnish dyslexic adults (Mittag, Alho, et al., 2013).

A similar pattern of results or relation with behavior (reading fluency) was not observed in the asynchronous crossmodal (AV200) blocks, possibly indicating a diminished recruitment of speech specific integration processes in this condition. This may also explain why typical adult readers, with selectively tuned neural systems for letter-speech sound integration, only show congruency effects when letters and speech sounds are presented simultaneously (AV0 blocks, Froyen et al., 2008; van Atteveldt et al., 2004). These findings deserve further study however, especially in relation to the nature of possible audiovisual integration deficits in dyslexics, because in a previous study with 11 year old dyslexic readers, behavioral measures of word reading fluency (MMN), non-word reading fluency and letter-phoneme matching (LN) were found to correlate with MMN/LN letter effects in the AV200 condition (Froyen et al., 2011).

Further evidence for brain-behavior relations with respect to letter-speech sound integration comes mainly from fMRI studies. (Blau et al., 2010), for example found a positive correlation between letter-speech sound congruency effects measured in the planum temporale/Heschl's sulcus and both reading accuracy and the speed of letter-speech sound matching across 9-year old typical and dyslexic readers. Cross-modal fMRI congruency effects measured in the planum temporale during a rhyme judgment task were also found to positively correlate with literacy skills (McNorgan, Awati, Desroches, \& Booth, 2013) as well as with phonological awareness (McNorgan, Randazzo-Wagner, et al., 2013) in 8-11 year old typical readers. However, in the latter study no correlation was found between cross-modal integration and phonological awareness in dyslexic children, which was suggested to indicate a decoupling of these processes in dyslexia (McNorgan, Randazzo-Wagner, et al., 2013). Although most studies thus find a relation between reading and/or phonological skills and ERP/fMRI indices of letter-speech sound integration, the type of relation shows some variability as well as its presence or absence in 
dyslexic readers. These differences may relate to the dependency of neural effects of letter-speech sound congruency on task demands (Lafontaine et al., 2012; van Atteveldt, Formisano, Goebel, et al., 2007) the type of speech/letter units, e.g. consonants, vowels, syllables (Andres et al., 2011; Mittag, Thesleff, Laasonen, \& Kujala, 2013) and/or the depth of the orthography (Holloway, van Atteveldt, Blomert, \& Ansari, 2013). Importantly, the present results show that the severity of reading (dys)fluency may also lead to differences in the observed brain-behavior correlations across studies. It would thus be interesting to further investigate these brain-behavior relationships in a largerscale cross-national study including children of varying reading (dys)fluency, and deep and shallow orthographies, using the same paradigm with stimuli of grain sizes with different relevance in the different orthographies. Furthermore, the observed relation between reading fluency/accuracy and crossmodal ERP modulations across typical as well as dyslexic readers is compatible with a continuity model of reading dysfunction [80], although our results do not exclude the possibility of dyslexia subtypes.

Given the present focus on letter-speech sound integration, it may seem surprising that behavioral measures of letter-speech sound coupling showed the weakest differences across reading groups and did not correlate with ERP measures of letter-speech sound coupling. However, these findings are in agreement with previous ERP and fMRI evidence revealing a dissociation between children's knowledge of which letters belong to which speech sounds and the automatic neural integration of these associations (Blomert \& Vaessen, 2009; Booth et al., 2001; Froyen et al., 2009). The present results thus provide additional support for the notion that behavioral measures alone may not to be a sensitive indicator for letter-speech sound integration (Blomert, 2011; Froyen et al., 2009).

\subsection{Normal auditory responses and subtle reduction of $\mathrm{LN}$ letter effect in dyslexics}

Results showed comparable auditory MMN and LN responses to vowels in all three groups. This absence of group differences was expected because vowels 


\section{Reading Fluency and Cross-Modal ERPs in Dyslexia}

typically elicit normal MMN and/or LN responses in dyslexic children (Valéria Csépe, 2003; Froyen et al., 2011) whereas deficient change detection responses are typically reported for more subtle speech changes involving e.g. stop consonants (e.g. Valéria Csépe, 2003; Schulte-Körne, Deimel, Bartling, \& Remschmidt, 1998). Correspondingly, we did not find any correlations between auditory MMN and LN responses and behavioral measures of reading or reading-related skills.

Our results also confirm weak neural integration of letters and speech sounds in 9 year old dyslexic children (Blau et al., 2010), with reduced or absent audiovisual LN effects in both groups and a short-lived MMN effect in the AV0 condition in severely dysfluent dyslexics. The timing of these effects was different than those previously reported in 11 year old dyslexics, for whom MMN letter effects did not, but the LN letter effect in the AV200 condition did, reach significance (Froyen et al., 2011). These differences could relate to developmental or strategy changes in dyslexic readers after 2.5 versus 4 years of reading instruction, but could also relate to methodological differences between the two studies and/or differences in the severity of reading dysfluency (see above).

Interestingly, an attenuated late negativity to spoken / ba/ deviants as compared to $/ \mathrm{da} /$ standards has been proposed as a potential endophenotype for dyslexia (Neuhoff et al., 2012). That is, this negativity in a broader time window (300$700 \mathrm{~ms}$ ) overlapping with our crossmodal LN (600-750 ms) was found to be attenuated in both dyslexic children and their siblings without dyslexia (Neuhoff et al., 2012), and to be associated with rare variants in a candidate gene region for dyslexia (Czamara et al., 2011). As compared to perceptual aspects of letter-speech sound congruency in the MMN window, the observed LN letter effect may reflect cognitive, explicit associative and/or attentional processes (Froyen et al., 2009, 2011; Neuhoff et al., 2012; Shestakova, Huotilainen, Čeponiené, \& Cheour, 2003), present depending on familiarity and complexity of the stimuli. Whereas in adults these type of late orthographicphonological interactions may only occur during complex metaphonological 
tasks (Lafontaine et al., 2012; Pattamadilok et al., 2011) and pseudoword-word priming tasks (Savill \& Thierry, 2011), in typically reading children they seem to be recruited during the integration of simple letter-vowel pairs (present findings and Froyen et al., 2009), letter strings (Maurer, Brem, Bucher, \& Brandeis, 2005), integration of audiovisual words (Jost, Eberhard-Moscicka, Frisch, Dellwo, \& Maurer, 2013) and a visual lexical decision task with phonological distractors (Hasko et al., 2013), with disrupted recruitment in dyslexic children (present findings and Froyen et al., 2011; Hasko et al., 2013). If this process is disrupted, as was the case in both dyslexic groups then the automaticity in adulthood may not be reached (Blau et al., 2009), as it may be prevented by an incapability to access and/or manipulate the representations (Blau et al., 2010; Ramus \& Ahissar, 2012; Savill \& Thierry, 2011), and/or reduced attentionallymediated integration (Czamara et al., 2011; Neuhoff et al., 2012; Savill \& Thierry, 2011, 2012). The additional attenuation of the early MMN in severely dyslexic children may signal a more basic failure in forming a clear letter-speech sound representation (Blomert, 2011), and concurs with the suggested timing of sublexical orthographic-phonological integration during online visual word recognition (Grainger \& Holcomb, 2009). Similarly, Roeske and colleagues (Roeske et al., 2011) observed an association between specific genetic markers and a speech evoked late negativity across dyslexic children independently of the severity of their reading problems, while a significant association with the earlier speech evoked MMN was present only in the most severe dyslexics. Thus, the crossmodal MMN enhancement would represent a successful representation of an audiovisual stimulus, and the crossmodal LN enhancement would characterize availability of the represented stimulus for further manipulation.

Finally, Blau and colleagues (Blau et al., 2010) showed that Dutch dyslexic children of the same age as our participants, exhibit reduced neural integration of letters and speech sounds in the planum temporale/Heschl sulcus and the superior temporal sulcus, thus showing both reduced integration in the STS and feedback to the auditory cortex (van Atteveldt et al., 2004). As the severely dysfluent dyslexics in our study showed both early and late deficiencies, while 


\section{Reading Fluency and Cross-Modal ERPs in Dyslexia}

dysfluent dyslexics showed only a late deficiency, it would be interesting to investigate whether the early MMN deficiency would stem from the reduced integration in the STS while the LN deficiency would be a product of a reduced feedback.

\subsection{Conclusion}

The present ERP study investigated letter-speech sound integration in 9-yearold typically reading and dyslexic children by using a well-studied oddball paradigm with simple vowel sounds and letters and demonstrated that early (speech specific) audiovisual integration processes scale with individual differences in reading (dys)fluency. Results further indicated enhanced neural sensitivity to letter-speech sound associations in 9-year-old typical readers, together with disrupted sensitivity in dyslexic readers. In future studies, this ERP paradigm could be used to investigate whether and how systematic training of phonological skills and/or letter speech-sound coupling changes this disrupted neural integration of letters and speech sounds in dyslexic children. Furthermore, an extension to longitudinal designs using units of different complexity and grain sizes (e.g. vowels, consonants, syllables, words), possibly in the context of more naturalistic on-line reading tasks, could lead to a more complete understanding of the dynamic neural changes contributing to successful and hampered reading acquisition.

\section{Acknowledgements}

We dedicate this paper to our co-author, professor Leo Blomert, who passed away on November 25, 2012. His contributions to the project prior to his passing were significant.

This project is part of the research program "Fluent reading acquisition neurocognitively decomposed: the case of dyslexia (HCMI 10-59)" funded by the Netherlands Initiative Brain and Cognition (NIHC), a part of the Netherlands Organization for Scientific Research (NWO) under grant number 056-14-015. 
We thank Suzanne van Grieken, Marlena van Langevelde, Gert-Jan Munneke, Jitka Annen, Mandy Meijer, Helene Vos and Jorinde Wesseling for their help during data collection and Nienke van Atteveldt for useful comments on the manuscript. Last but not least, a special thanks goes to the children participating in the research and their parents.

\section{References}

Achenbach, T. M., \& McConaughy, S. H. (2003). The Achenbach System of Empirically Based Assessment (ASEBA). In C. R. Reynolds \& R. W. Kamphaus (Eds.), Handbook of psychological and educational assessment of children (2nd ed., pp. 406-430). New York, NY: Guilford Press.

Andres, A. J. D., Oram Cardy, J. E., \& Joanisse, M. F. (2011). Congruency of auditory sounds and visual letters modulates mismatch negativity and P300 event-related potentials. International Journal of Psychophysiology : Official Journal of the International Organization of Psychophysiology, 79(2), 137-46. doi:10.1016/j.ijpsycho.2010.09.012

Annett, M. (1979). Family handedness in three generations predicted by the right shift theory. Annals of Human Genetics, 42(4), 479-491. doi:10.1111/j.1469-1809.1979.tb00681.x

Aravena, S., Snellings, P., Tijms, J., \& van der Molen, M. W. W. (2013). A lab-controlled simulation of a letter-speech sound binding deficit in dyslexia. Journal of Experimental Child Psychology, 115(4), 691-707. doi:10.1016/j.jecp.2013.03.009

Baart, M., Stekelenburg, J. J., \& Vroomen, J. (2014). Electrophysiological evidence for speechspecific audiovisual integration. Neuropsychologia, 53, 115-21. doi:10.1016/j.neuropsychologia.2013.11.011

Beauchamp, M. S., Lee, K. E., Argall, B. D., \& Martin, A. (2004). Integration of auditory and visual information about objects in superior temporal sulcus. Neuron, 41(5), 809-23. Retrieved from http://www.ncbi.nlm.nih.gov/pubmed/15003179

Benjamini, Y., \& Hochberg, Y. (1995). Controlling the false discovery rate: a practical and powerful approach to multiple testing. Journal of the Royal Statistical Society. Series B (Methodological), 57(1), 289-300. Retrieved from http://www.jstor.org/stable/10.2307/2346101

Blau, V., Reithler, J., van Atteveldt, N., Seitz, J., Gerretsen, P., Goebel, R., \& Blomert, L. (2010). Deviant processing of letters and speech sounds as proximate cause of reading failure: a functional magnetic resonance imaging study of dyslexic children. Brain: A Journal of Neurology, 133(Pt 3), 868-79. doi:10.1093/brain/awp308

Blau, V., van Atteveldt, N., Ekkebus, M., Goebel, R., \& Blomert, L. (2009). Reduced neural integration of letters and speech sounds links phonological and reading deficits in adult dyslexia. Current Biology : CB, 19(6), 503-8. doi:10.1016/j.cub.2009.01.065 


\section{Reading Fluency and Cross-Modal ERPs in Dyslexia}

Blomert, L. (2005). Dyslexie in Nederland. Amsterdam: Uitgeverij Nieuwezijds. Amsterdam: Uitgeverij Nieuwezijds. Retrieved from http://www.boomtestuitgevers.nl/upload/Dyslexie_in_Nederland_Leo_Blomert.pdf

Blomert, L. (2011). The neural signature of orthographic-phonological binding in successful and failing reading development. NeuroImage, 57(3), 695-703. doi:10.1016/j.neuroimage.2010.11.003

Blomert, L., \& Vaessen, A. A. (2009). 3DM Differential diagnostics for dyslexia: Cognitive analysis of reading and spelling. Amsterdam: Boom Test Publishers.

Boersma, P., \& Weenink, D. (2002). Praat: doing phonetics by computer (Version 4.0. 26). Amsterdam: University of Amsterdam. Retrieved from http://www.fon.hum.uva.nl/praat.

Bonte, M. L., \& Blomert, L. (2004). Developmental dyslexia: ERP correlates of anomalous phonological processing during spoken word recognition. Brain Research. Cognitive Brain Research, 21(3), 360-76. doi:10.1016/j.cogbrainres.2004.06.010

Bonte, M. L., Mitterer, H., Zellagui, N., Poelmans, H., \& Blomert, L. (2005). Auditory cortical tuning to statistical regularities in phonology. Clinical Neurophysiology: Official Journal of the International Federation of Clinical Neurophysiology, 116(12), 2765-74. doi:10.1016/j.clinph.2005.08.012

Bonte, M. L., Poelmans, H., \& Blomert, L. (2007). Deviant neurophysiological responses to phonological regularities in speech in dyslexic children. Neuropsychologia, 45(7), 1427-37. doi:10.1016/j.neuropsychologia.2006.11.009

Booth, J. R., Burman, D. D., Van Santen, F. W., Harasaki, Y., Gitelman, D. R., Parrish, T. B., \& Marsel Mesulam, M. M. (2001). The development of specialized brain systems in reading and oral-language. Child Neuropsychology : A Journal on Normal and Abnormal Development in Childhood and Adolescence, 7(3), 119-41. doi:10.1076/chin.7.3.119.8740

Brus, B. T., \& Voeten, M. J. M. (1973). Eén-minuut test, vorm A en B; Verantwoording en Handleiding. Nimegen: Berkenhout Testmateriaal.

Bus, A. G., \& Ijzendoorn, M. H. Van. (1999). Phonological Awareness and Early Reading : A Meta-Analysis of Experimental Training Studies. Journal of Educational Psychology, 91(3), 403-414. doi:10.1037/0022-0663.91.3.403

Cheour, M., Korpilahti, P., Martynova, O., \& Lang, a H. (2001). Mismatch negativity and late discriminative negativity in investigating speech perception and learning in children and infants. Audiology \& Neuro-Otology, 6(1), 2-11. doi:46804

Cheour, M., Shestakova, A., Alku, P., Ceponiene, R., \& Näätänen, R. (2002). Mismatch negativity shows that 3 - 6-year-old children can learn to discriminate non-native speech sounds within two months. Neuroscience Letters, 325, 187-190.

Cheour-Luhtanen, M., Alho, K., Kujala, T., Sainio, K., Reinikainen, K., Renlund, M., ... Näätänen, R. (1995). Mismatch negativity indicates vowel discrimination in newborns. Hearing Research, 82, 53-58. Retrieved from 
http://www.sciencedirect.com/science/article/pii/037859559400164L

Colin, C., Radeau, M., Soquet, a, Demolin, D., Colin, F., \& Deltenre, P. (2002). Mismatch negativity evoked by the McGurk-MacDonald effect: a phonetic representation within short-term memory. Clinical Neurophysiology: Official Journal of the International Federation of Clinical Neurophysiology, 113(4), 495-506. Retrieved from http://www.ncbi.nlm.nih.gov/pubmed/11955994

Csépe, V. (1995). On the origin and development of the mismatch negativity. Ear and Hearing, 16(1), 91-104. Retrieved from http://scholar.google.com/scholar?hl=en\&btnG=Search\&q=intitle:On+the+origin + and + development + of + the + mismatch + negativity $\# 0$

Csépe, V. (2003). Auditory event-related potentials in studying developmental dyslexia. In V. Csépe (Ed.), Dyslexia: Different brain, different behavior (pp. 81-112). New York, NY: Kluwer Academic Publishers - Plenum Publishers.

Czamara, D., Bruder, J., Becker, J., Bartling, J., Hoffmann, P., Ludwig, K. U., ... Schulte-Körne, G. (2011). Association of a rare variant with mismatch negativity in a region between KIAA0319 and DCDC2 in dyslexia. Behavior Genetics, 41(1), 110-9. doi:10.1007/s10519010-9413-6

de Vos, T. (2007). Schoolvaardigheidstoets Technisch Lezen. Amsterdam: Boom test uitgevers.

Delorme, A., \& Makeig, S. (2004). EEGLAB: an open source toolbox for analysis of single-trial EEG dynamics including independent component analysis. Journal of Neuroscience Methods, 134, 9-21. Retrieved from http://www.sciencedirect.com/science/article/pii/S0165027003003479

Ehri, L. C. (2005). Learning to Read Words: Theory, Findings, and Issues. Scientific Studies of Reading, 9(2), 167-188. doi:10.1207/s1532799xssr0902_4

Eulitz, C., \& Lahiri, A. (2004). Neurobiological Evidence for Abstract Phonological Representations in the Mental Lexicon during Speech Recognition. Journal of Cognitive Neuroscience, 16(4), 577-583.

Faulkner, H. J., \& Levy, B. a. (1999). Fluent and nonfluent forms of transfer in reading: words and their message. Psychonomic Bulletin \& Review, 6(1), 111-6. Retrieved from http://www.ncbi.nlm.nih.gov/pubmed/12199304

Froyen, D., Bonte, M. L., van Atteveldt, N., \& Blomert, L. (2009). The long road to automation: neurocognitive development of letter-speech sound processing. Journal of Cognitive Neuroscience, 21(3), 567-80. doi:10.1162/jocn.2009.21061

Froyen, D., Van Atteveldt, N., Bonte, M., \& Blomert, L. (2008). Cross-modal enhancement of the MMN to speech-sounds indicates early and automatic integration of letters and speech-sounds. Neuroscience Letters, 430(1), 23-8. doi:10.1016/j.neulet.2007.10.014

Froyen, D., Willems, G., \& Blomert, L. (2011). Evidence for a specific cross-modal association deficit in dyslexia: an electrophysiological study of letter-speech sound processing. 


\section{Reading Fluency and Cross-Modal ERPs in Dyslexia}

Developmental Science, 14(4), 635-48. doi:10.1111/j.1467-7687.2010.01007.x

Fuchs, D., Fuchs, L. S., \& Compton, D. L. (2004). Identifying Reading Disabilities by Responsiveness-to-Instruction: Specifying Measures and Criteria. Learning Disability Quarterly, 27(4), 216-227. doi:10.2307/1593674

Grainger, J., \& Holcomb, P. J. (2009). Watching the Word Go by: On the Time-course of Component Processes in Visual Word Recognition. Language and Linguistics Compass, 3(1), 128-156. doi:10.1111/j.1749-818X.2008.00121.x

Hasko, S., Bruder, J., Bartling, J., \& Schulte-Körne, G. (2012). N300 indexes deficient integration of orthographic and phonological representations in children with dyslexia. Neuropsychologia, 50(5), 640-54. doi:10.1016/j.neuropsychologia.2012.01.001

Hasko, S., Groth, K., Bruder, J., Bartling, J., \& Schulte-Körne, G. (2013). The time course of reading processes in children with and without dyslexia: an ERP study. Frontiers in Human Neuroscience, 7(October), 570. doi:10.3389/fnhum.2013.00570

Holloway, I. D., van Atteveldt, N., Blomert, L., \& Ansari, D. (2013). Orthographic Dependency in the Neural Correlates of Reading: Evidence from Audiovisual Integration in English Readers. Cerebral Cortex (New York, N.Y. : 1991). doi:10.1093/cercor/bht347

Hommet, C., Vidal, J., Roux, S., Blanc, R., Barthez, M. A., De Becque, B., ... Gomot, M. (2009). Topography of syllable change-detection electrophysiological indices in children and adults with reading disabilities. Neuropsychologia, 47(3), 761-70. doi:10.1016/j.neuropsychologia.2008.12.010

Jost, L. B., Eberhard-Moscicka, A. K., Frisch, C., Dellwo, V., \& Maurer, U. (2013). Integration of Spoken and Written Words in Beginning Readers: A Topographic ERP Study. Brain Topography, 27, 786-800. doi:10.1007/s10548-013-0336-4

Jung, T. P., Makeig, S., Humphries, C., Lee, T. W., McKeown, M. J., Iragui, V., \& Sejnowski, T. J. (2000). Removing electroencephalographic artifacts by blind source separation. Psychophysiology, 37(2), 163-78. Retrieved from http://www.ncbi.nlm.nih.gov/pubmed/10731767

Kasai, K., Nakagome, K., Iwanami, A., Fukuda, M., Itoh, K., Koshida, I., \& Kato, N. (2002). No effect of gender on tonal and phonetic mismatch negativity in normal adults assessed by a high-resolution EEG recording. Brain Research. Cognitive Brain Research, 13(3), 305-12. Retrieved from http://www.ncbi.nlm.nih.gov/pubmed/11918996

Katzir, T., Kim, Y.-S. Y.-S., Wolf, M., Morris, R., \& Lovett, M. W. (2008). The Varieties of Pathways to Dysfluent Reading: Comparing Subtypes of Children With Dyslexia at Letter, Word, and Connected Text Levels of Reading. Journal of Learning Disabilities, 41(1), 47-66. doi:10.1177/0022219407311325

Knowland, V. C. P., Mercure, E., Karmiloff-Smith, A., Dick, F., \& Thomas, M. S. C. (2014). Audio-visual speech perception: a developmental ERP investigation. Developmental Science, 17(1), 110-24. doi:10.1111/desc.12098 
Lafontaine, H., Chetail, F., Colin, C., Kolinsky, R., \& Pattamadilok, C. (2012). Role and activation time course of phonological and orthographic information during phoneme judgments. Neuropsychologia, 50(12), 2897-906. doi:10.1016/j.neuropsychologia.2012.08.020

Lee, T. W., Girolami, M., \& Sejnowski, T. J. (1999). Independent component analysis using an extended infomax algorithm for mixed subgaussian and supergaussian sources. Neural Computation, 11(2), 417-41. Retrieved from http://www.ncbi.nlm.nih.gov/pubmed/9950738

Leinonen, S., Müller, K., Leppänen, P. H. T., Aro, M., Ahonen, T., \& Lyytinen, H. (2001). Heterogeneity in adult dyslexic readers : Relating processing skills to the speed and accuracy of oral text reading *. Reading and Writing, 14(3-4), 265-296. doi:10.1023/A:1011117620895

Liebenthal, E., Desai, R., Ellingson, M. M., Ramachandran, B., Desai, A., \& Binder, J. R. (2010). Specialization along the left superior temporal sulcus for auditory categorization. Cerebral Cortex (New York, N.Y. : 1991), 20(12), 2958-70. doi:10.1093/cercor/bhq045

Lohvansuu, K., Hämäläinen, J. a, Tanskanen, A., Bartling, J., Bruder, J., Honbolygó, F., ... Leppänen, P. H. T. (2013). Separating mismatch negativity (MMN) response from auditory obligatory brain responses in school-aged children. Psychophysiology, 50(7), 640-52. doi:10.1111/psyp.12048

Luck, S. J. (2005). An introduction to the event-related potential technique. Cambridge, MA: The MIT Press.

Lyon, G. R., Shaywitz, S. E., \& Shaywitz, B. A. (2003). A definition of dyslexia. Annals of Dyslexia, 53(1), 1-14. doi:10.1007/s11881-003-0001-9

Maurer, U., Brem, S., Bucher, K., \& Brandeis, D. (2005). Emerging neurophysiological specialization for letter strings. Journal of Cognitive Neuroscience, 17(10), 1532-52. doi:10.1162/089892905774597218

Maurer, U., Brem, S., Kranz, F., Bucher, K., Benz, R., Halder, P., ... Brandeis, D. (2006). Coarse neural tuning for print peaks when children learn to read. NeuroImage, 33(2), 749-58. doi:10.1016/j.neuroimage.2006.06.025

McNorgan, C., Awati, N., Desroches, A. S., \& Booth, J. R. (2013). Multimodal Lexical Processing in Auditory Cortex Is Literacy Skill Dependent. Cerebral Cortex (New York, N.Y. : 1991). doi: $10.1093 /$ cercor/bht100

McNorgan, C., Randazzo-Wagner, M., \& Booth, J. R. (2013). Cross-modal integration in the brain is related to phonological awareness only in typical readers, not in those with reading difficulty. Frontiers in Human Neuroscience, 7(July), 388. doi:10.3389/fnhum.2013.00388

Mittag, M., Alho, K., Takegata, R., Makkonen, T., \& Kujala, T. (2013). Audiovisual attention boosts letter-speech sound integration. Psychophysiology, 50, 1034-1044. doi:10.1111/psyp.12085

Mittag, M., Takegata, R., \& Kujala, T. (2011). The effects of visual material and temporal 


\section{Reading Fluency and Cross-Modal ERPs in Dyslexia}

synchrony on the processing of letters and speech sounds. Experimental Brain Research. Experimentelle Hirnforschung. Expérimentation Cérébrale, 211(2), 287-98. doi:10.1007/s00221011-2686-z

Mittag, M., Thesleff, P., Laasonen, M., \& Kujala, T. (2013). The neurophysiological basis of the integration of written and heard syllables in dyslexic adults. Clinical Neurophysiology : Official Journal of the International Federation of Clinical Neurophysiology, 124(2), 315-26. doi:10.1016/j.clinph.2012.08.003

Näätänen, R. (2001). The perception of speech sounds by the human brain as reflected by the mismatch negativity (MMN) and its magnetic equivalent (MMNm). Psychophysiology, 38(1), $1-21$.

Näätänen, R., Paavilainen, P., Rinne, T., \& Alho, K. (2007). The mismatch negativity (MMN) in basic research of central auditory processing: a review. Clinical Neurophysiology : Official Journal of the International Federation of Clinical Neurophysiology, 118(12), 2544-90. doi:10.1016/j.clinph.2007.04.026

Nath, A. R., \& Beauchamp, M. S. (2012). A neural basis for interindividual differences in the McGurk effect, a multisensory speech illusion. NeuroImage, 59(1), 781-7. doi:10.1016/j.neuroimage.2011.07.024

Nath, A. R., Fava, E. E., \& Beauchamp, M. S. (2011). Neural correlates of interindividual differences in children's audiovisual speech perception. The Journal of Neuroscience: The Official Journal of the Society for Neuroscience, 31(39), 13963-71. doi:10.1523/JNEUROSCI.2605-11.2011

Neuhoff, N., Bruder, J., Bartling, J., Warnke, A., Remschmidt, H., Müller-Myhsok, B., \& SchulteKörne, G. (2012). Evidence for the late MMN as a neurophysiological endophenotype for dyslexia. PloS One, 7(5), e34909. doi:10.1371/journal.pone.0034909

Oades, R. D., Dmittmann-Balcar, A., \& Zerbin, D. (1997). Development and topography of auditory event-related potentials (ERPs): Mismatch and processing negativity in individuals 8?22 years of age. Psychophysiology, 34(6), 677-693. doi:10.1111/j.14698986.1997.tb02143.x

Pattamadilok, C., Perre, L., \& Ziegler, J. C. (2011). Beyond rhyme or reason: ERPs reveal taskspecific activation of orthography on spoken language. Brain and Language, 116(3), 116-24. doi:10.1016/j.bandl.2010.12.002

Pilling, M. (2009). Auditory event-related potentials (ERPs) in audiovisual speech perception. Journal of Speech, Language, and Hearing Research : JSLHR, 52(4), 1073-81. doi:10.1044/10924388(2009/07-0276)

Ponton, C. W., Eggermont, J. J., Kwong, B., \& Don, M. (2000). Maturation of human central auditory system activity: evidence from multi-channel evoked potentials. Clinical Neurophysiology: Official Journal of the International Federation of Clinical Neurophysiology, 111(2), 220-36. Retrieved from http://www.ncbi.nlm.nih.gov/pubmed/10680557

Raij, T., Uutela, K., \& Hari, R. (2000). Audiovisual integration of letters in the human brain. 
Neuron, 28(2), 617-25. Retrieved from http://www.ncbi.nlm.nih.gov/pubmed/11144369

Ramus, F., \& Ahissar, M. (2012). Developmental dyslexia: the difficulties of interpreting poor performance, and the importance of normal performance. Cognitive Neuropsychology, 29(1-2), 104-22. doi:10.1080/02643294.2012.677420

Ramus, F., \& Szenkovits, G. (2008). What phonological deficit? Quarterly Journal of Experimental Psychology (2006), 61(1), 129-41. doi:10.1080/17470210701508822

Raven, J., Raven, J. C., \& Court, J. H. (1998). Coloured progressive matrices. Oxford, UK: Oxford Psychologists Press.

Roeske, D., Ludwig, K. U., Neuhoff, N., Becker, J., Bartling, J., Bruder, J., ... Schulte-Körne, G. (2011). First genome-wide association scan on neurophysiological endophenotypes points to trans-regulation effects on SLC2A3 in dyslexic children. Molecular Psychiatry, 16(1), 97107. doi:10.1038/mp.2009.102

Savill, N. J., \& Thierry, G. (2011). Reading for sound with dyslexia: evidence for early orthographic and late phonological integration deficits. Brain Research, 1385, 192-205. doi:10.1016/j.brainres.2011.02.012

Savill, N. J., \& Thierry, G. (2012). Decoding ability makes waves in reading: deficient interactions between attention and phonological analysis in developmental dyslexia. Neuropsychologia, 50(7), 1553-64. doi:10.1016/j.neuropsychologia.2012.03.008

Schulte-Körne, G., Deimel, W., Bartling, J., \& Remschmidt, H. (1998). Auditory processing and dyslexia: evidence for a specific speech processing deficit. Neuroreport, 9(2), 337-340.

Shafer, V. L., Morr, M. L., Kreuzer, J. A., \& Kurtzberg, D. (2000). Maturation of mismatch negativity in school-age children. Ear and Hearing, 21(3), 242-51. Retrieved from http:/ / cat.inist.fr/ ?aModele $=$ afficheN\&cpsidt $=1410810$

Share, D. L. (1995). Phonological recoding and self-teaching: sine qua non of reading acquisition. Cognition, 55(2), 151-218; discussion 219-26. Retrieved from http://www.ncbi.nlm.nih.gov/pubmed/7789090

Shaywitz, S. E., Morris, R., \& Shaywitz, B. A. (2008). The education of dyslexic children from childhood to young adulthood. Annual Review of Psychology, 59, 451-75.

doi:10.1146/annurev.psych.59.103006.093633

Shestakova, A., Huotilainen, M., Čeponienè, R., \& Cheour, M. (2003). Event-related potentials associated with second language learning in children. Clinical Neurophysiology, 114(8), 15071512. doi:10.1016/S1388-2457(03)00134-2

Snowling, M. J. (1998). Dyslexia as a Phonological Deficit: Evidence and Implications. Child Psychology and Psycbiatry Review, 3(1), 4-11. doi:10.1017/S1360641797001366

Stanovich, K. E., Feeman, D. J., \& Cunningham, A. E. (1983). The development of the relation between letter-naming speed and reading ability. Bulletin of the Psychonomic Society, 21(3), 199-202. doi:10.3758/BF03334686 


\section{Reading Fluency and Cross-Modal ERPs in Dyslexia}

Stekelenburg, J. J., Vroomen, J., \& de Gelder, B. (2004). Illusory sound shifts induced by the ventriloquist illusion evoke the mismatch negativity. Neuroscience Letters, 357(3), 163-6. doi:10.1016/j.neulet.2003.12.085

Tijms, J., \& Hoeks, J. (2005). A computerized treatment of dyslexia: benefits from treating lexicophonological processing problems. Dyslexia (Chichester, England), 11(1), 22-40. doi:10.1002/dys.283

Vaessen, A., Bertrand, D., Tóth, D., Csépe, V., Faísca, L., Reis, A., \& Blomert, L. (2010). Cognitive development of fluent word reading does not qualitatively differ between transparent and opaque orthographies. Journal of Educational Psychology, 102(4), 827-842. doi:10.1037/a0019465

van Atteveldt, N., Blau, V. C., Blomert, L., \& Goebel, R. (2010). fMR-adaptation indicates selectivity to audiovisual content congruency in distributed clusters in human superior temporal cortex. BMC Neuroscience, 11, 11. doi:10.1186/1471-2202-11-11

van Atteveldt, N., Formisano, E., Blomert, L., \& Goebel, R. (2007). The effect of temporal asynchrony on the multisensory integration of letters and speech sounds. Cerebral Cortex (New York, N.Y.: 1991), 17(4), 962-74. doi:10.1093/cercor/bhl007

van Atteveldt, N., Formisano, E., Goebel, R., \& Blomert, L. (2004). Integration of letters and speech sounds in the human brain. Neuron, 43(2), 271-82. doi:10.1016/j.neuron.2004.06.025

van Atteveldt, N., Formisano, E., Goebel, R., \& Blomert, L. (2007). Top-down task effects overrule automatic multisensory responses to letter-sound pairs in auditory association cortex. NeuroImage, 36(4), 1345-60. doi:10.1016/j.neuroimage.2007.03.065

van Wassenhove, V., Grant, K. W., \& Poeppel, D. (2005). Visual speech speeds up the neural processing of auditory speech. Proceedings of the National Academy of Sciences of the United States of America, 102(4), 1181-6. doi:10.1073/pnas.0408949102

Wimmer, H., \& Schurz, M. (2010). Dyslexia in regular orthographies: manifestation and causation. Dyslexia, 16(4), 283-299. doi:10.1002/dys.411

Winkler, I., Kujala, T., Tiitinen, H., Sivonen, P., Alku, P., Lehtokoski, A., ... Näätänen, R. (1999). Brain responses reveal the learning of foreign language phonemes. Psychophysiology, 36(5), 638-42. Retrieved from http://www.ncbi.nlm.nih.gov/pubmed/10442032 


\section{CHAPTER 3}

\section{Crossmodal deficit in dyslexic children: practice affects the neural timing of letter-speech sound integration}

Corresponding publication:

Žarić, G., Fraga González, G., Tijms, J., van der Molen, M. W., Blomert, L., and Bonte, M. (2015). Crossmodal deficit in dyslexic children: practice affects the neural timing of letter-speech sound integration. Frontiers in Human Neuroscience, 369. 


\begin{abstract}
A failure to build solid letter-speech sound associations may contribute to reading impairments in developmental dyslexia. Whether this reduced neural integration of letters and speech sounds changes over time within individual children and how this relates to behavioral gains in reading skills remains unknown. In this research, we examined changes in event-related potential (ERP) measures of letter-speech sound integration over a 6-month period during which 9-year-old dyslexic readers $(n=17)$ followed a training in letter-speech sound coupling next to their regular reading curriculum. We presented the Dutch spoken vowels /a/ and /o/ as standard and deviant stimuli in one auditory and two audiovisual oddball conditions. In one audiovisual condition (AV0), the letter 'a' was presented simultaneously with the vowels, while in the other (AV200) it was preceding vowel onset for 200 ms. Prior to the training (T1), dyslexic readers showed the expected pattern of typical auditory mismatch responses, together with the absence of letter-speech sound effects in a late negativity (LN) window. After the training (T2), our results showed earlier (and enhanced) crossmodal effects in the LN window. Most interestingly, earlier LN latency at T2 was significantly related to higher behavioral accuracy in letter-speech sound coupling. On a more general level, the timing of the earlier mismatch negativity (MMN) in the simultaneous condition (AV0) measured at $\mathrm{T}$ 1, significantly related to reading fluency at both T1 and T2 as well as with reading gains. Our findings suggest that the reduced neural integration of letters and speech sounds in dyslexic children may show moderate improvement with reading instruction and training and that behavioral improvements relate especially to individual differences in the timing of this neural integration.
\end{abstract}




\section{Introduction}

Although most children learn to read fluently, between $5 \%$ and $10 \%$ of children are diagnosed with developmental dyslexia exhibiting deficient reading skills despite normal cognitive abilities and schooling opportunities (Blomert, 2005; Lyon, Shaywitz, \& Shaywitz, 2003; Snowling, 2013). The formation of letter-speech sound pairs, an important first step in obtaining reading expertise in alphabetic orthographies, forms an immediate obstacle for beginner readers with dyslexia (Blomert, 2011; Ehri, 2005; Share, 1995; Wimmer \& Schurz, 2010). Consequently, many dyslexia interventions include modules focused on teaching letter-speech sound correspondences (Aravena, Snellings, Tijms, \& van der Molen, 2013; Bus \& Ijzendoorn, 1999; Tijms \& Hoeks, 2005) next to dealing with impaired phonological processing (Ramus \& Szenkovits, 2008; Snowling, 1998). Accumulating evidence from neuroimaging studies suggests reduced neural integration of letters and speech sounds in dyslexic children (Blau et al., 2010; Froyen, Willems, \& Blomert, 2011; McNorgan, RandazzoWagner, \& Booth, 2013; Žarić et al., 2014) and adults (Blau, van Atteveldt, Ekkebus, Goebel, \& Blomert, 2009). However a remaining open question is whether an increased exposure to literacy and especially learning letter-speech sound correspondences can change the neural integration deficit in dyslexia.

In transparent orthographies such as Dutch, children typically learn to accurately identify and discriminate letter-speech sound pairs during the first year of reading instruction (Blomert \& Vaessen, 2009). This is contrasted by the results of neuroimaging studies in which development of automatic neural integration shows a much more protracted period throughout primary school (Booth et al., 2001; Froyen, Bonte, van Atteveldt, \& Blomert, 2009). This prolonged maturation of the neural integration of letters and speech sounds has been observed in studies measuring EEG responses in a passive crossmodal "oddball" paradigm (Froyen et al., 2009; Froyen, Van Atteveldt, Bonte, \& Blomert, 2008; Froyen et al., 2011; Žarić et al., 2014). In oddball paradigms a mismatch negativity (MMN) response is elicited at $100-250 \mathrm{~ms}$ after presentation of a so called oddball or deviant sound within a train of frequent (standard) sounds. The MMN is believed to reflect an automatic 
response to deviation from traces formed in auditory short-term memory due to the frequent repetition of the standard stimulus (Näätänen, Paavilainen, Rinne, \& Alho, 2007; Näätänen, 2001). The MMN response is sensitive to both language-specific speech sounds and audiovisual integration in children and adults (Andres, Oram Cardy, \& Joanisse, 2011; Bonte, Poelmans, \& Blomert, 2007; Marie Cheour, Shestakova, Alku, Ceponiene, \& Näätänen, 2002; Cheour-Luhtanen et al., 1995; V Csépe, 1995; Froyen et al., 2009, 2008; Kasai et al., 2002; Lohvansuu et al., 2013; Mittag, Alho, Takegata, Makkonen, \& Kujala, 2013; Mittag, Takegata, \& Kujala, 2011; Näätänen, 2001; Neuhoff et al., 2012; Žarić et al., 2014). Additionally, a broader late negativity (Late MMN or LN), between 300 to $700 \mathrm{~ms}$ after deviant onset, can be seen in school-aged children, while it is diminished in adults (M Cheour, Korpilahti, Martynova, \& Lang, 2001; Czamara et al., 2011; Froyen et al., 2008; Hommet et al., 2009), suggesting the recruitment of additional processing resources in children.

The crossmodal oddball paradigm (Froyen et al., 2009, 2008, 2011; Žarić et al., 2014) consists of an auditory and two audiovisual experimental conditions during which the Dutch speech sounds /a/ and /o/ are presented as the standard and deviant stimuli respectively. In the audiovisual blocks, the letter ' $a$ ' is paired with the speech sounds resulting in a double mismatch: the deviant speech sound /o/ is incongruent both with respect to the standard speech sound /a/ and the presented letter ' $a$ ' (Fig. 3-1). The stimulus onset asynchrony (SOA) between the letter and the speech sound is either 0 or 200 $\mathrm{ms}$ to allow investigation of the temporal window of integration. In adults, this paradigm showed an early MMN enhancement due to the presentation of the letter and only with synchronous letter-speech sound pairs (Froyen et al., 2008), while children additionally showed later LN letter effects and a developmental shift in the temporal integration window (Froyen et al., 2009; Žarić et al., 2014). In agreement with an inverted ' $U$ ' trajectory of increased brain responses during early school years followed by reduced and more selective responses with age and experience (Bonte \& Blomert, 2004; Maurer et al., 2006), the MMN and LN of 9 year old typical readers exhibited stronger crossmodal effects than in younger ( 8 year old) as well as older (11 year old) 
readers and adults. In particular, 9-year old children showed enhanced crossmodal MMN and LN responses to both synchronous and asynchronous letter speech sound pairs (Žarić et al., 2014). Furthermore, although adults did not show the later $\mathrm{LN}$ in this passive paradigm using simple speech stimuli (Froyen et al., 2008), they have been reported to show late orthographicphonological interactions in spoken language processing (400-700 ms) during more complex metaphonological tasks (Lafontaine, Chetail, Colin, Kolinsky, \& Pattamadilok, 2012; Pattamadilok, Perre, \& Ziegler, 2011). It can thus be speculated that while the crossmodal MMN enhancement reflects early and automatic letter-speech sound integration and/or representation (Näätänen et al., 2007; Näätänen, 2001), the crossmodal LN enhancement reflects more elaborate associative processes that are recruited for the integration of simple letter-speech pairs during initial learning phases, but become redundant once this integration becomes automatic and overlearned.

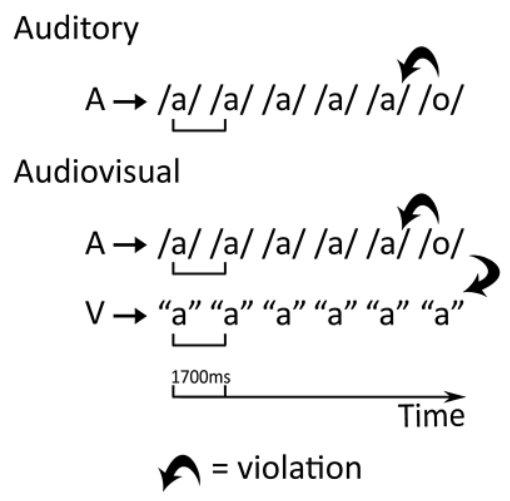

Figure 3-1. Design of the auditory and the audiovisual experiment. A - auditory stimulus, $V$ - visual stimulus. The thick bent arrows represent the violation of the standard speech sound in the auditory experiment and the violation of both the standard speech sound and the letter in the audiovisual experiment

In dyslexic children, the crossmodal oddball paradigm indicated typical vowelevoked change detection responses together with reduced crossmodal effects (Froyen et al., 2011; Žarić et al., 2014). In particular, all 9 year old dyslexic participants showed a reduction in the crossmodal LN effect independently of 
the synchronicity of the letter-speech sound pairs, while the MMN response to synchronous letter-speech sound pairs was reduced only in the most severely dysfluent dyslexics (Žarić et al., 2014). Moreover, MMN latency in the synchronous condition was coupled with individual differences in reading fluency (Žarić et al., 2014). Further evidence comes from fMRI studies in 9year-old children and adults with dyslexia demonstrating reduced neural integration of letters and speech sounds in superior temporal cortical regions (Blau et al., 2010, 2009). EEG studies in dyslexic adults additionally reported the absence of a crossmodal MMN enhancement for spoken-written syllable pairs (Mittag et al., 2013) as well as reduced orthographic-phonological integration during word reading tasks (Hasko, Bruder, Bartling, \& SchulteKörne, 2012; Hasko, Groth, Bruder, Bartling, \& Schulte-Körne, 2013; Savill \& Thierry, 2011, 2012). Interestingly, a recent behavioral study (Aravena et al., 2013) employing an artificial script for letter-speech sound learning showed that children with dyslexia reached comparable levels of letter-speech sound knowledge to their typical reading peers, while remaining less fluent in mapping of letter-speech sound pairs. Thus, knowledge of letter-speech sound pairs by itself is not sufficient for the automation of letter-speech sound integration, and dyslexics may exhibit a specific deficit in the automation of this integration.

Here we employ the passive crossmodal oddball paradigm (Froyen et al., 2009, 2008, 2011; Žarić et al., 2014) to investigate whether 9-year old dyslexic children exhibit changes in crossmodal EEG responses over time and whether these changes relate to behavioral improvements in reading fluency and letterspeech sound coupling. To this end, we examine MMN and LN measures of letter-speech sound integration in the same children after 2,5 (T1) and 3 years (T2) of reading instruction, spanning a 6 months period during which they followed the first part of a systematic cognitive reading intervention program focusing on letter-speech sound integration (Tijms, 2004, 2007, 2011). The 17 children that were followed in the present study are a subgroup of the 36 dyslexic children that participated in a previous study (Žarić et al., 2014) and includes those that came back for EEG and behavioral measurements at T2. 
As we collected EEG and behavioral data at both measurements, we examine the relation between ERP and behavioral measures at T1 based on our previous study (Žarić et al., 2014), as well as the predictive power of these ERP measures with respect to behavioral change, and the relation between changes in ERP and behavioral measures following the training.

\section{Methods}

\subsection{Participants}

In total, 19 dyslexic children participated in a first (T1) and second (T2) EEG session after six months. All children were native Dutch speakers, with 2.5 years of reading instruction at T1 and 3 years of reading instruction, including 34 sessions of reading intervention (Tijms, 2004, 2007, 2011) at T2. Data of 17 children were included in the analysis (on average 9.0 years old at T1, range: 8.2-9.9 years; 12 girls) Three children were left-handed, as assessed with a modified version of Annett's handedness questionnaire (Annett, 1979). Data of 2 children were discarded, one due to the malfunction of recording reference electrodes and the other due to excessive movements during the first EEG measurement.

All children included in the study were diagnosed as dyslexic after an extensive cognitive psycho-diagnostic procedure at a specialized institute for dyslexia and reading problems (IWAL institute Amsterdam) and scored lower than the 10th percentile of the age appropriate group on standard reading tests (see below). Other behavioral scores such as phonological skills or rapid automatized naming (RAN) were not used as selection criteria for the current study. Parents reported normal hearing and normal or corrected to normal vision for all children. Parents also completed the Child Behavior Checklist (CBCL), a part of the Achenbach system of empirically based assessment (ASEBA) (Achenbach \& McConaughy, 2003). Exclusion criteria included comorbidity with behavioral and/or attention disorders (assessed with the CBCL) and below average IQ. Written informed consent was obtained from the parents of each child. We reimbursed travel costs and each child was given a small present after each session of the experiment. The approval for the research was 
obtained from the ethical committee of the Developmental Psychology department of the University of Amsterdam (Ethics file 2011-OP-1907) and the research was conducted according to the relevant regulatory standards.

\subsection{Behavioral tests}

Preceding the first EEG measurement, children underwent individual testing on standard language tests including the one-minute word reading test (EMT; Brus \& Voeten, 1973), and reading of a short story 'De kat' ('The cat'; de Vos, 2007) as well as subtests of the Dyslexia Differential Diagnosis - 3DM battery (Blomert \& Vaessen, 2009), assessing word reading, spelling, letter-speech sound identification, letter-speech sound discrimination, rapid automized naming (RAN) and basic reaction time. Additionally, we assessed non-verbal IQ scores by means of a paper and pencil version of the RAVEN Coloured Progressive Matrices (RAVEN CPM; Raven, Raven, \& Court, 1998). At the time of the second EEG measurement children performed a shorter version of the behavioral test battery including the word reading, spelling, letter-speech sound identification and letter-speech sound discrimination subtests of the 3DM battery, EMT and 'De kat'. Subject characteristics and the results of the behavioral tests are shown in Table 3-1. For each test that was completed at both measurements, differences between the two measurements were tested using repeated measures ANOVA (see Results section).

\subsection{Reading instruction and Training}

Next to their regular reading curriculum at school, all children took part in a training program (Tijms, 2004, 2007, 2011) that was provided twice per week on a one-to-one basis with each session lasting 45 minutes. Training was performed with a computer-assisted training program and guided by a tutor. Sessions consisted of an instruction and a practice part. First, letter-speech sound correspondences were explicitly trained during the instruction part, with the aim to achieve a mastery level, i.e. at least $80 \%$ of the items correctly executed at each step. Second, during the practice part, a high exposure to the trained letter speech sound associations was provided to stimulate the automatic integration of letters and speech sounds. The letter-speech sound 
correspondences were taught in a step-by-step fashion, increasing the level of complexity, e.g. from the short vowels to the diphthongs and starting with the regular letter-speech sound correspondences before continuing with the irregular ones. The computer training progressed within time constraints, which were adapted to the individual performance of each child.

\subsection{Stimuli}

The stimuli and experimental design were the same as in our previous study (Žarić et al., 2014) involving digital recordings of a native Dutch female speaker pronouncing the Dutch vowels /a/ and /o/ (sampling rate $44.1 \mathrm{kHz}$, 16 bit quantization; band-pass filtered: 180-10.000 Hz; downsampled to 22.05 $\mathrm{kHz}$ and matched for loudness using Praat software; Boersma \& Weenink, 2002). Sound duration was $384 \mathrm{~ms}$ for vowel /a/ and $348 \mathrm{~ms}$ for vowel /o/. The vowel sounds were presented to both ears via headphones with a loudness of $\sim 65 \mathrm{~dB}$ as measured with an analog loudness meter. The letter stimulus consisted of a white "a" presented in lower case "Arial" font, size 40, in the center of a black computer screen. Presentation 14.4 (Neurobehavioral Systems, Inc., Albany, CA) was used for stimulus presentation.

\subsection{Experimental Design}

The standard stimulus, vowel /a/, was presented in $83 \%$ of trials while the deviant stimulus, vowel /o/, was presented in $17 \%$ of the trials, in one auditory and two audiovisual oddball conditions (Fig. 1). In the two audiovisual conditions, the letter " $a$ " was presented together with the vowels for $500 \mathrm{~ms}$. Between successive letter presentations, a white fixation cross appeared in the same location. Trial length was always $1700 \mathrm{~ms}$. The difference between the two audiovisual conditions involved the stimulus onset asynchrony (SOA) between the presentation of the letter and vowel stimuli. In the synchronous audiovisual condition (AV0), the letter and vowel appeared simultaneously, while the letter appeared $200 \mathrm{~ms}$ before the vowel in the asynchronous audiovisual condition (AV200). To ensure that participants fixated on the screen during the audiovisual conditions, we employed a simple visual target detection task in which they had to press a button when a target (color picture 
of a wrapped present) was presented instead of the letter (10 trials per block). For each condition, three blocks of 288 trials were presented in succession, summing to a total of 714 standard and 150 deviant (17\%) trials. Standards and deviants were pseudo-randomized in each block: at least three standards were separating two successive deviants and deviants could not occur in the first two trials of a block. The order of the conditions was pseudorandomized and counterbalanced, with one of the crossmodal conditions always being presented first.

\subsection{EEG data recording and analysis}

The Biosemi Active Two system (Biosemi, Amsterdam, Netherlands) was used for EEG data recording. EEG was measured from 64 active-channels, placed according to the 10-20 international system (Electro-cap International Inc.). The CMS electrode was used as the recording reference and was placed at the approximate location of PO1 (the DRL electrode was placed at the approximate location of PO2). Four additional Flat-Type Active electrodes of which two were placed below and above the left eye and two at the outer canthi of each eye were used to measure eye-movements. Two additional electrodes were placed on the right and left mastoids and used for offline rereferencing. Sampling rate of recording was $1024 \mathrm{~Hz}$ with a DC-104 Hz bandwidth. The offset range of the electrodes was kept between $-20 \mathrm{mV}$ and $20 \mathrm{mV}$.

The first two trials of each block and the first trial after each deviant were excluded from the analysis. Additionally, the first two trials after catch trials in the audiovisual conditions were not included in the analysis to avoid movement artifacts due to button presses (Luck, 2005). For each subject we randomly selected 150 of the remaining 480 standard trials for the further analysis, together with the 150 deviant trials. Analysis was performed with the EEGLAB toolbox (v11.0.0b (Delorme \& Makeig, 2004) http://www.sccn.ucsd.edu/eeglab) and MATLAB 2014a, (The MathWorks, Natick, MA). We rereferenced data offline to the average of the left and right mastoids. Data was further bandpass filtered $(1-70 \mathrm{~Hz})$ and downsampled to 
256Hz. EEG epochs were extracted with respect to the onset of the auditory stimulus in all conditions, corresponding to epochs of $-500 \mathrm{~ms}$ to $1200 \mathrm{~ms}$ in the Auditory and AV0 conditions and of $-700 \mathrm{~ms}$ to $1000 \mathrm{~ms}$ in the AV200 condition. Baseline correction was performed with respect to the mean signal in the $500 \mathrm{~ms}$ baseline period prior to stimulation corresponding to $-500 \mathrm{~ms}$ to $0 \mathrm{~ms}$ in the Auditory and AV0 conditions and $-700 \mathrm{~ms}$ to $-200 \mathrm{~ms}$ in the AV200 condition, thereby avoiding activation due to the letter in the AV200 in the baseline period. For visualization purposes all ERPs are shown for $-500 \mathrm{~ms}$ to $1000 \mathrm{~ms}$ with respect to the auditory onset. We used a two-step protocol for artifact removal. First, we manually rejected the epochs containing nonstereotypical artifacts (e.g. electrode cable movements, rare jaw clinching). See Table 3-2 for the mean (SD) number of epochs included in the averages for the standards and deviants after this initial artifact rejection step.

In the second step, we employed extended INFOMAX ICA (Lee, Girolami, \& Sejnowski, 1999) on 64 scalp channels resulting in 64 components per condition per participant. ICA is optimal for identifying repeating stereotypical signals, including, for example, artifacts such as eye blinks, eye movements and muscle artifacts (e.g. swallowing) that constitute the major source of noise in EEG data of children. Independent components (ICs) were categorized as EEG activity or stereotypical non-brain artifacts based on visual inspection of their characteristic scalp topographies, time courses and power-frequency spectra (Jung et al., 2000). Components were classified as EEG activity according to the following criteria (1) scalp topography indicating an underlying dipolar source, (2) spectral peaks typical of EEG, and (3) a regular occurrence across single trials (Delorme \& Makeig, 2004). ICs representing non-brain artifacts were removed, and EEG data were reconstructed from the remaining component activations. See Table 3-2 for the mean (SD) number of components per subject in the different conditions. Single electrodes containing high amplitude noise were interpolated using spherical interpolation after the data was reconstructed. The reconstructed data was baseline corrected and low pass filtered at $30 \mathrm{~Hz}$. Event related potentials (ERPs) were calculated for each participant by averaging the epochs per stimulus and condition. 


\begin{tabular}{|c|c|c|c|c|c|c|c|}
\hline \multirow{3}{*}{ 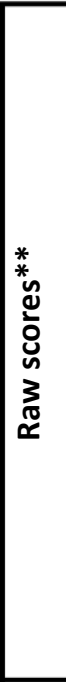 } & & $\begin{array}{l}\sigma \\
\frac{\sigma}{-1} \\
\frac{-1}{4}\end{array}$ & 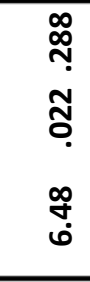 & 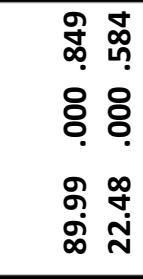 & $\begin{array}{l}\text { \& } \\
\text {. } \\
8 \\
8 \\
0 \\
0 \\
\dot{0} \\
\text { d }\end{array}$ & 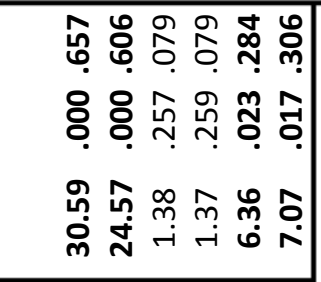 & 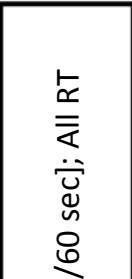 \\
\hline & 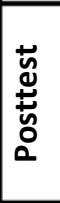 & 요 & 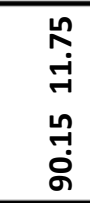 & 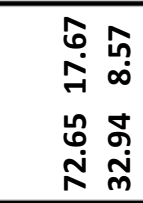 & \begin{tabular}{l}
$\underset{N}{N}$ \\
$\infty$ \\
\multirow{1}{*}{} \\
\multirow{\infty}{\infty}{}
\end{tabular} & 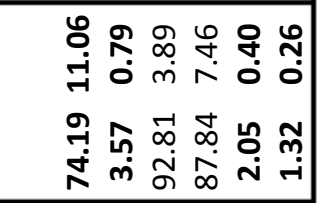 & $\begin{array}{l}\frac{n}{0} \\
\frac{0}{0} \\
3 \\
z \\
\frac{1}{\pi} \\
\frac{\pi}{2}\end{array}$ \\
\hline & 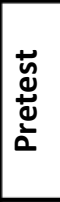 & 요 & 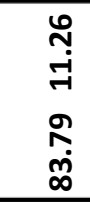 & 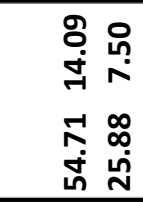 & 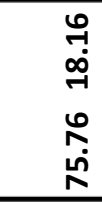 & 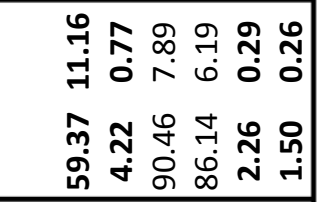 & 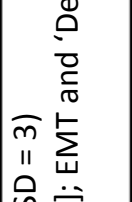 \\
\hline \multirow{4}{*}{ 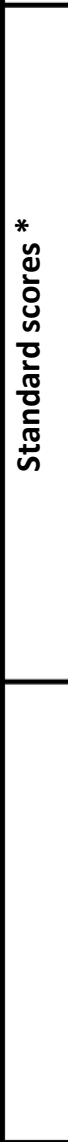 } & & $\begin{array}{l}\sigma \\
\stackrel{\sigma}{-} \\
\frac{-1}{4}\end{array}$ & 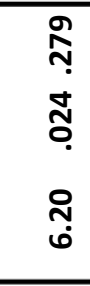 & 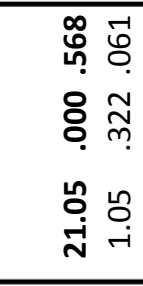 & 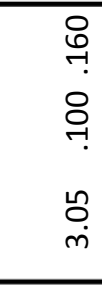 & 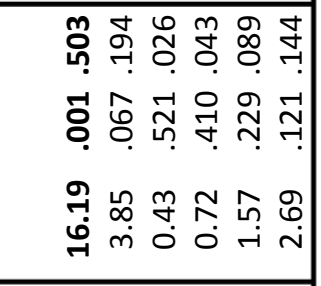 & 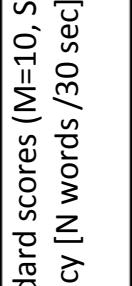 \\
\hline & 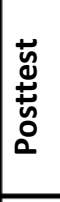 & $\Sigma$ & 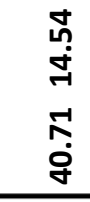 & 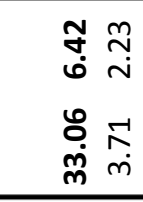 & $\begin{array}{l}\infty \\
\infty \\
m \\
m\end{array}$ & 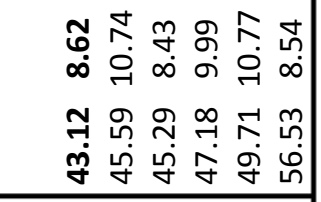 & 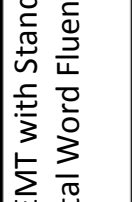 \\
\hline & 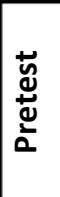 & 오 & 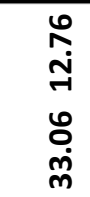 & 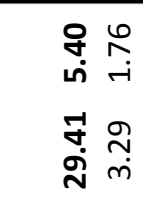 & 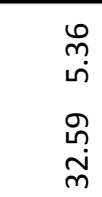 & 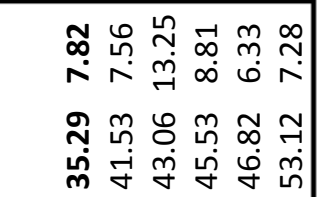 & 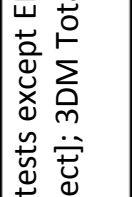 \\
\hline & & & 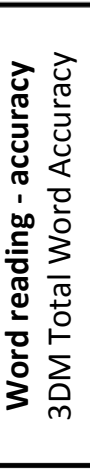 & 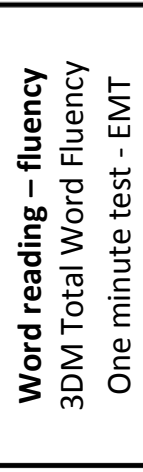 & 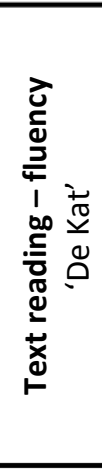 & 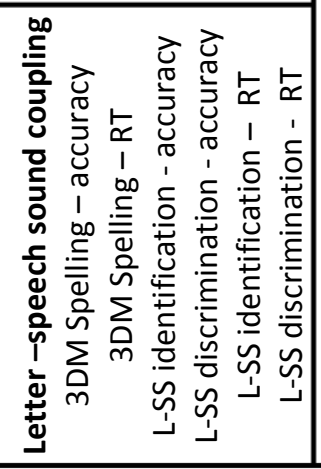 & 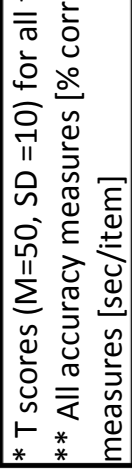 \\
\hline
\end{tabular}


We analyzed ERPs evoked by standard and deviant vowel sounds in the auditory and both audiovisual conditions at each of our measurements sessions to evaluate the presence of the mismatch negativity (MMN) and late negativity $(\mathrm{LN})$. As we aimed to study how these auditory mismatch responses are influenced by the presentation of visual letters, we focused our analysis on fronto-central sites at which these responses are known to be maximal (Bishop, 2007; Kujala, Lovio, Lepistö, Laasonen, \& Näätänen, 2006; Näätänen et al., 2007; Neuhoff et al., 2012). In particular, we included seven electrodes, $\mathrm{Fz}, \mathrm{FCz}, \mathrm{Cz}, \mathrm{F} 3, \mathrm{~F} 4, \mathrm{FC} 3, \mathrm{FC} 4$, covering the maximal MMN and $\mathrm{LN}$ responses in the auditory and crossmodal conditions at both measurement times. Thus, maximal MMN responses occurred at FCz (Auditory, AV0 at T1, Auditory, Av0, AV200 at T2) and FC3 (AV200 at T1) and maximal LN responses occurred at FCz (AV at T2), FC2 (Av200 at T1), Fz (AV0 at T1 and Auditory at T2), Cz (Auditory at T1) and F4 (AV200 at T2). Note that an analysis including the 4 fronto-central electrodes used in our previous study (Žarić et al., 2014), yielded the same pattern of results as the analysis including these seven electrodes. Individual $\mathrm{MMN}$ and $\mathrm{LN}$ peak latencies were manually determined within their respective time windows: 100 - $250 \mathrm{~ms}$ for the MMN and $600-750 \mathrm{~ms}$ for the LN (Froyen et al., 2009, 2011; Žarić et al., 2014). Mean amplitude across $50 \mathrm{~ms}$ centered on the individual peak latencies was used as the amplitude measure of the MMN and LN responses. Using SPSS, Version 21.0 (IBM Corp. Armonk, NY), we first applied repeated measures ANOVAs with stimulus (2 levels: standard and deviant) and electrode (7 levels) as within subjects factors per time window, per condition per measurement, to evaluate the presence of the MMN and LN responses. We subtracted the ERPs evoked by standards from those of deviants to obtain difference waves used in the analysis of crossmodal effects. We first performed repeatedmeasures ANOVA with condition (3 levels: Au, AV0, AV200) and electrode (7 levels) as within subjects factors for the first measurement (T1) with the aim of testing whether the pattern of auditory and crossmodal ERP effects as previously observed in a larger group of 36 dyslexic children that participated at T1 (Žarić et al., 2014) would be preserved in the current subsample of 17 dyslexic children that participated at both T1 and T2. We then also performed 
this analysis at T2. In case of significant condition effects, we performed posthoc analysis of MMN/LN amplitude and latency measures on pairs of conditions (Au-AV0; Au-AV200) with condition (2 levels) and electrode (7 levels) as within subject factors. Furthermore, we compared the amplitudes and latencies at the two measurements by including the within subject factors test (2 levels: T1 and T2), condition (amplitude, 3 levels: Au, AV0, AV200; latency, 2 levels: AV0, Av200) and electrode (7 levels) in the repeated measures ANOVA. We report Greenhouse-Geisser corrected p-values for the factors for which the assumption of sphericity was violated. In addition to significance values, as in our previous study (Žarić et al., 2014) we report effect sizes as represented by partial eta squared $\left(\eta_{\mathrm{p}}{ }^{2}\right)$ as this measure is more comparable between studies than classical eta squared (Lakens, 2013). In a further analysis, following our previous findings (Žarić et al., 2014), we analyzed the N1 and P2 responses to standard and deviant stimuli in the synchronous crossmodal condition (AV0) using repeated measures ANOVAs with stimulus (2 levels: standard, deviant) and electrode (7 levels) as within subject factors. All posthoc tests were corrected for multiple comparisons by Bonferroni correction. Correction was employed for the two condition comparisons investigating crossmodal enhancement as compared to the auditory condition: AV0 vs. Auditory and AV200 versus Auditory, resulting in a corrected $\alpha_{\text {Bonferroni }}=.025$.

\begin{tabular}{|c|c|c|c|c|c|}
\hline & & & Auditory & AVo & AV200 \\
\hline \multirow{2}{*}{ Number of retained ICs } & \multicolumn{2}{|c|}{ T1 } & $44(7)$ & $45(7)$ & $45(7)$ \\
\hline & \multicolumn{2}{|c|}{ T2 } & $42(6)$ & $46(6)$ & $45(8)$ \\
\hline \multirow{4}{*}{ Number of retained trials } & \multirow{2}{*}{ T1 } & $\mathbf{S}$ & $148(4)$ & $149(1)$ & $147(2)$ \\
\hline & & D & $148(5)$ & $148(1)$ & $147(3)$ \\
\hline & \multirow{2}{*}{ T2 } & $\mathbf{S}$ & $148(2)$ & $148(3)$ & $148(2)$ \\
\hline & & D & $148(2)$ & $147(2)$ & $148(3)$ \\
\hline
\end{tabular}


We employed linear regression to investigate how individual differences in ERP correlates of letter-sound associations relate to behavioral measures of reading fluency, reading accuracy and letter-speech sound coupling. To test replicability of the results from the first study (Žarić et al., 2014), we regressed MMN latency in the synchronous (AV0) condition on behavioral scores during the first measurement (T1). To test predictive power of this MMN latency on later behavioral scores, we analyzed its relation with behavioral measures from the second measurement (T2) as well as with behavioral gains from T1 to T2. Furthermore, as the LN latency in the crossmodal conditions significantly decreased from $\mathrm{T} 1$ to $\mathrm{T} 2$, also the relation between these measures and behavioral measures at T2 was analyzed. For linear regression, ERP latencies were computed as composite scores across 7 fronto-central electrodes. Following our previous study behavioral measures were quantified in terms of composite scores for reading fluency (3DM word reading tests, the EMT, and "De Kat"), reading accuracy (3DM), letter-speech sound coupling accuracy (spelling, letter-speech sound identification and discrimination,) and speed (spelling, letter-speech sound identification and discrimination) (Vaessen et al., 2010; Žarić et al., 2014). To examine specific relations between latencies and behavior we used simple linear regressions with one of the ERP latencies as a predictor and one of the behavioral composite scores as a dependent variable. We used false discovery rate (FDR) correction (Benjamini \& Hochberg, 1995), as we conducted 12 tests for MMN AV0 latency and 8 tests for LN AV0 and AV200 latencies and report $\mathrm{p}$ values relative to $\mathrm{q}(\mathrm{FDR})<.05$ thresholds. Finally, to partial out autoregressive effects when testing MMN latency at T1 and reading fluency at T2, we employed linear regression with both $\mathrm{MMN}$ latency and reading fluency at T1 as predictors.

\section{Results}

\subsection{Behavioral tests}

A comparison of behavioral test scores in the dyslexic children at the first (T1) and second (T2) measurements showed significant improvements at the group level on the raw scores of all tests, except for letter-speech sound identification and discrimination accuracies (Table 3-1). Moreover, results showed significant 
group-level increases in age-normed scores on the 3DM word reading tests (fluency and accuracy), as well as on 3DM spelling accuracy, demonstrating a greater improvement than would be expected just based on the time elapsed. At the group level both word reading fluency and accuracy were significantly impaired at T1 (below the 10th percentile, t-score of 37), while at T2 word reading accuracy reached the non-impaired range. At the level of individual children, word reading fluency improved with time and in 5 out of 17 children reached the non-impaired range at T2 (above the 10th percentile, t-score of 37). Reading accuracy scores also improved with 6 children scoring in the nonimpaired range at T1 and 10 children at T2. Note that this pattern of results is consistent with the finding that in relatively shallow orthographies such as Dutch, reading fluency is the most persistent deficit in dyslexia (Blomert, 2011; Wimmer \& Schurz, 2010; Ziegler, Perry, Ma-Wyatt, Ladner, \& Schulte-Körne, 2003).

\subsection{Auditory MMN and LN effects}

We first analyzed the MMN and $\mathrm{LN}$ responses evoked in the auditory condition as they served as a baseline for crossmodal enhancement effects, At both measurement times, the vowel deviant elicited an auditory MMN (Fig. 32) with the expected fronto-central topographical distribution (Fig. 3-3), leading to main effects of stimulus at $\mathrm{T} 1\left(\mathrm{~F}(1,16)=20.93, \mathrm{p}<.001, \eta_{\mathrm{p}}{ }^{2}=.567\right)$ and $\mathrm{T} 2\left(\mathrm{~F}(1,16)=47.59, \mathrm{p}<.001, \eta^{2}=.748\right)$. At both measurement times, an auditory $\mathrm{LN}$ response was elicited around $670-685 \mathrm{~ms}$ after stimulus onset with main effects of stimulus at $\mathrm{T} 1\left(\mathrm{~F}(1,16)=15.22, \mathrm{p}=.001, \eta_{\mathrm{p}}{ }^{2}=.488\right)$ and $\mathrm{T} 2$ $\left(\mathrm{F}(1,16)=11.82, \mathrm{p}=.003, \eta_{\mathrm{p}}{ }^{2}=.425\right) . \mathrm{A}$ comparison of auditory $\mathrm{MMN}$ and $\mathrm{LN}$ responses measured at $\mathrm{T} 1$ and $\mathrm{T} 2$, showed no significant changes between measurement times in terms of response amplitude or latency. 
Chapter 3

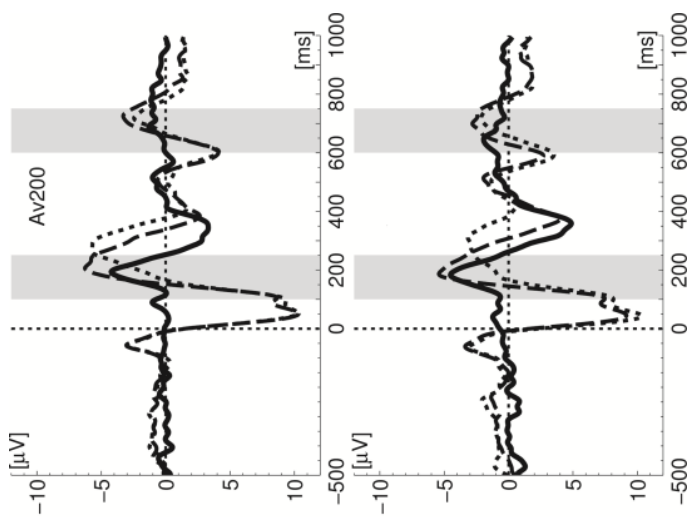

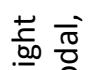

तิ

은

สิ

E

苋

है

光

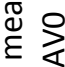

$\sum_{+2}$
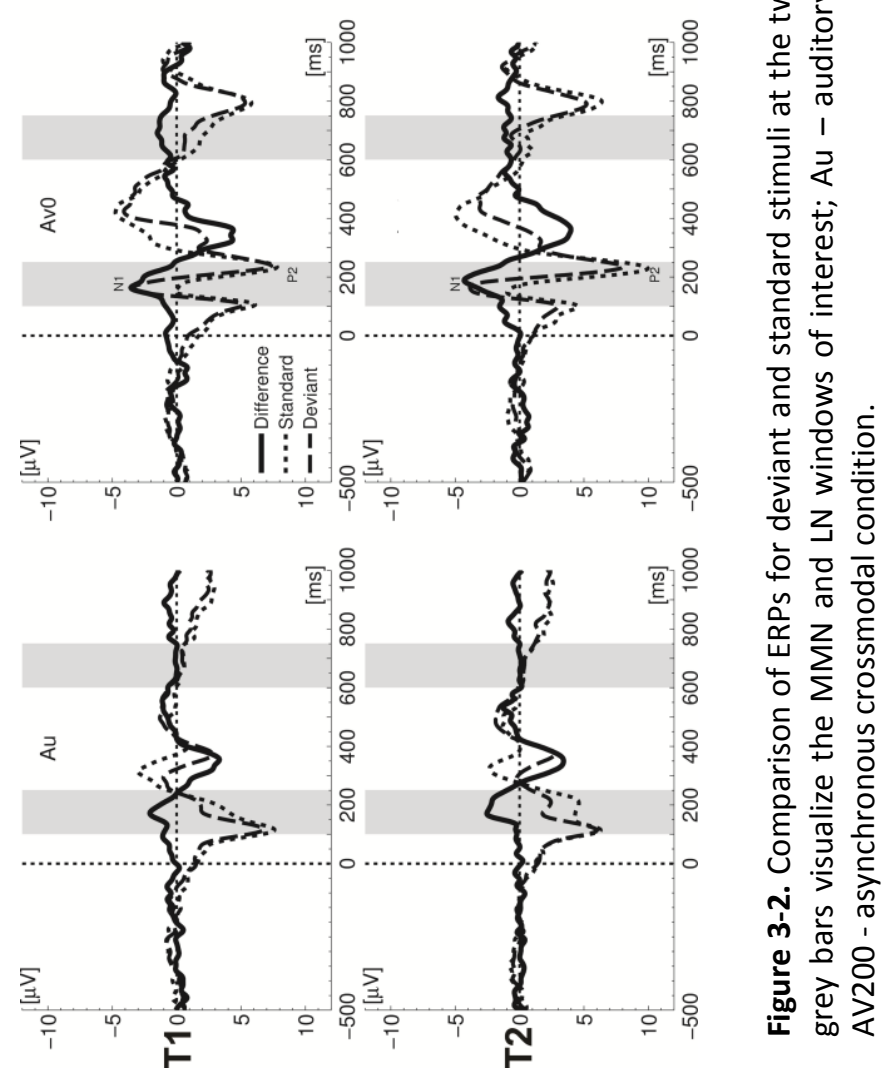


\subsection{Letter-effects: crossmodal MMN and $L N$ amplitudes}

The deviant vowel sound /o/ elicited MMN and $\mathrm{LN}$ responses in both crossmodal conditions (Fig. 3-2 and Fig. 3-3). One crucial quantification of actual crossmodal processing involves testing the effect of the double violation due to the presentation of the letter ' $\mathrm{a}$ ' in the crossmodal conditions. In other words, did the deviancy of the vowel sound /o/ from both the standard vowel sound / $\mathrm{a} /$ and the letter ' $\mathrm{a}$ ' significantly enhance the MMN and/or LN responses as compared to the auditory only condition (Froyen et al., 2009, 2008; Žarić et al., 2014). To this end, we performed repeated measures ANOVAs on average MMN and $\mathrm{LN}$ amplitudes extracted from ERP difference waves over 7 fronto-central electrodes, with condition (auditory, AV0, AV200) and electrode (7 levels) as within-subject factors.

We first compared the auditory and crossmodal conditions at T1 to test the pattern of amplitude enhancements in the smaller sample of dyslexic children that participated both at T1 and T2 as compared to a larger sample that only participated at T1 (Žarić et al., 2014). In the MMN window, this analysis yielded a main effect of condition $\left(\mathrm{F}(2,32)=3.93, \mathrm{p}=.030, \eta_{\mathrm{p}}^{2}=.197\right)$. A comparison of crossmodal conditions with the auditory condition revealed a significant crossmodal enhancement in the asynchronous AV200 condition $\left(\mathrm{F}(1,16)=9.64, \mathrm{p}=.007, \eta_{\mathrm{p}}{ }^{2}=.376\right)$ while this enhancement did not reach significance in the simultaneous AV0 condition $(\mathrm{F}(1,16)=4.62, \mathrm{p}=.047$, $\left.\eta_{\mathrm{p}}^{2}=.224\right)$ after Bonferroni correction $(\alpha=.025)$. In the $\mathrm{LN}$ window, dyslexic children showed no significant crossmodal enhancement (main effect of condition $\left(\mathrm{F}(2,32)=1.26, \mathrm{p}=.297, \eta_{\mathrm{p}}{ }^{2}=.073\right)$. This pattern of a significant crossmodal MMN enhancement in the AV200 condition, together with the absence of this enhancement in the AV0 condition and of later LN enhancements in both conditions, resembles the pattern of results of the most severely dysfluent children in a larger sample of dyslexics (Žarić et al., 2014). In particular, in this larger group of dyslexics, the most severely dysfluent children, did not show a significant MMN enhancement in the AV0 condition. Ten of the current 17 children belonged to this severely dysfluent group, which explains the absence of a significant MMN enhancement in this condition. 
As our previous results showed that the reduced crossmodal MMN response in the AVO condition in severely dysfluent dyslexics was due to a significant letter-speech sound deviancy effect in the N1 but not in the P2 window, we also separately analyzed these two responses. Results showed the same pattern of results in the current subsample at T1, with a significant crossmodal deviancy effect in the $\mathrm{N} 1\left(\mathrm{~F}(1,16)=20.45, \mathrm{p}<.001, \eta_{\mathrm{p}}{ }^{2}=.561\right)$, but not in the P2 window.

An analysis of MMN and LN enhancements measured 6 months later, at T2, yielded a marginally significant effect of condition in the MMN window $\left(\mathrm{F}(2,32)=3.23, \mathrm{p}=.052, \eta_{\mathrm{p}}^{2}=.168\right) . \quad \mathrm{A}$ comparison of separate conditions showed that this effect was due to a significant crossmodal MMN enhancement in the AV200 condition $\left(F(1,16)=7.39, \mathrm{p}=.015, \eta_{\mathrm{p}}{ }^{2}=.316\right)$ but not in the AV0 condition. In contrast to T1, at T2, a significant main effect of condition was observed in the $\mathrm{LN}$ window $\left(\mathrm{F}(2,32)=7.18, \mathrm{p}=.003, \eta_{\mathrm{p}}{ }^{2}=.310\right)$. The crossmodal LN response was significantly enhanced in both the AV0 $\left(\mathrm{F}(1,16)=7.09, \mathrm{p}=.017, \quad \eta_{\mathrm{p}}^{2}=.307\right)$ and $\operatorname{AV} 200 \quad(\mathrm{~F}(1,16)=13.34, \mathrm{p}=.002$, $\left.\eta_{\mathrm{p}}{ }^{2}=.455\right)$ conditions as compared to the auditory condition $\left(\alpha_{\text {Bonferroni }}=.025\right)$. A direct comparison of the two measurements did not yield significant differences between $\mathrm{T} 1$ and $\mathrm{T} 2$ for either the MMN or the $\mathrm{LN}$ response amplitudes. That is, the ANOVA with test (2 levels), condition (3 levels) and electrode ( 7 levels) as repeated measures did not yield significant main effects of test or test*condition interactions for the MMN/LN amplitudes. However, unlike at T1 (and Žarić et al., 2014), this overall analysis did yield significant main effects of condition for both the $\mathrm{MMN}(\mathrm{F}(2,32)=10.95, \mathrm{p}<.001$, $\left.\eta_{\mathrm{p}}{ }^{2}=.406\right)$ and $\mathrm{LN}\left(\mathrm{F}(2,32)=7.55, \mathrm{p}=.002, \eta_{\mathrm{p}}{ }^{2}=.321\right)$ amplitudes.

A separate analysis of $\mathrm{N} 1$ and P2 deviancy effects at T2 showed a significant mismatch response $\left(\alpha_{\text {Bonferroni }}=.025\right)$ in both the $\mathrm{N} 1(\mathrm{~F}(1,16)=14.10, \mathrm{p}=.002$, $\left.\eta_{\mathrm{p}}{ }^{2}=.468\right)$ and $\mathrm{P} 2$ windows $\left(\mathrm{F}(1,16)=9.08, \mathrm{p}=.008, \eta_{\mathrm{p}}{ }^{2}=.362\right)$. There was a tendency for an increase in the mismatch response from $\mathrm{T} 1$ to $\mathrm{T} 2$ in the P2 window (test: $\mathrm{F}(1,16)=3.09, \mathrm{p}=.098, \eta_{\mathrm{p}}^{2}=.162$; stimulus: $\mathrm{F}(1,16)=9.71$, 
$\left.\mathrm{p}=.007, \eta_{\mathrm{p}}{ }^{2}=.378\right)$, while there was no difference between T1 and T2 in the N1 window (stimulus: $\mathrm{F}(1,16)=34.93, \mathrm{p}<.001, \eta_{\mathrm{p}}{ }^{2}=.686$ ).

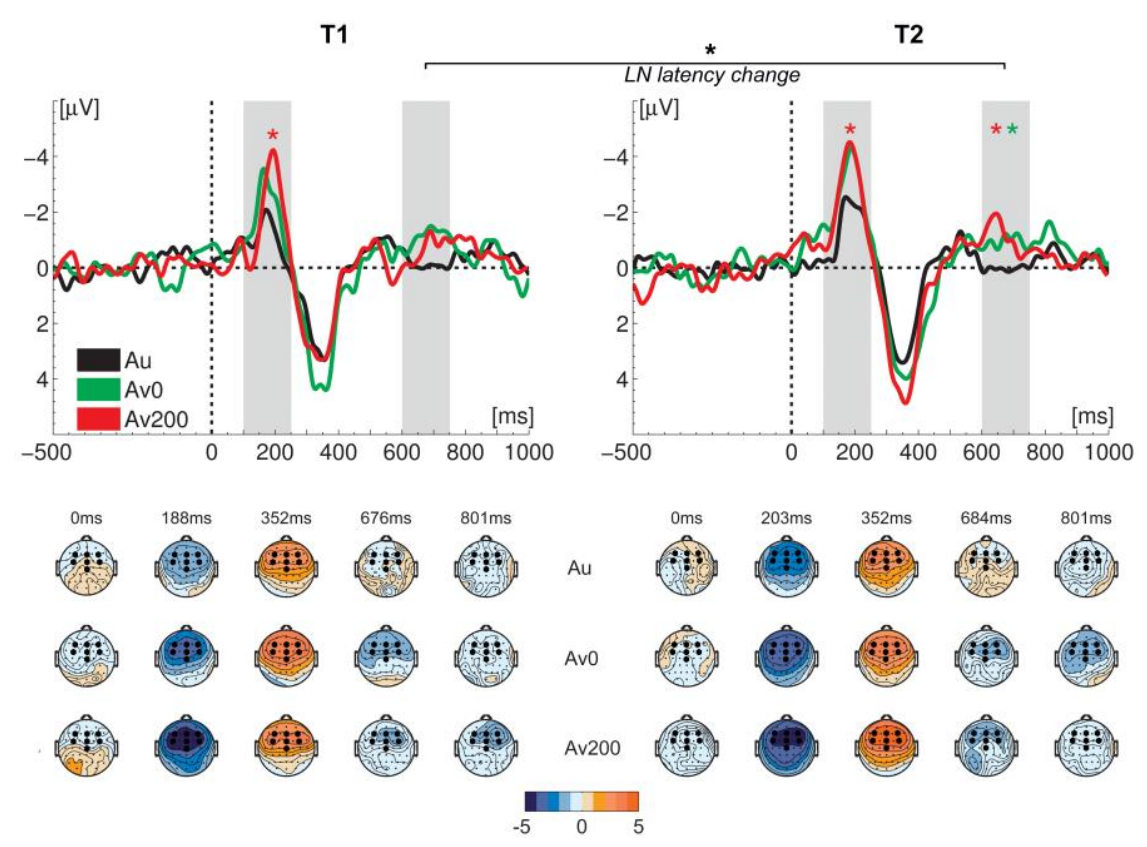

Figure 3-3. Difference waves (upper row) and topographical maps (lower row) at the two measurement times (T1 and T2); light grey bars visualize the MMN and LN time windows of interest; green and red asterisks indicate a significant MMN/LN amplitude enhancement in respectively the synchronous (AV0) and asynchronous (AV200) crossmodal conditions relative to the auditory condition (Au); black asterisk indicates a significant crossmodal LN latency difference from T1 to T2. Amplitude measures did not show significant differences from T1 to T2. Black hexagons in topographical maps represent the 7 fronto-central electrodes used in the analysis.

\subsection{Letter effects: MMN and LN latency}

The timing of the letter effects was investigated by analysis of MMN and LN latencies in both crossmodal conditions. The ANOVA with test (2 levels), condition ( 2 levels) and electrode ( 7 levels) as repeated measures yielded no difference in the timing of the crossmodal MMN responses between measurement times. Most interestingly, we did observe a shortening of the LN 
latency from $\mathrm{T} 1$ to $\mathrm{T} 2\left(\mathrm{~F}(1,16)=7.37, \mathrm{p}=.015, \eta_{\mathrm{p}}{ }^{2}=.315\right)$ without a significant main effect of condition or test*condition interaction.

\subsection{ERP - behavior relations}

In a further analysis we investigated how (changes in) MMN and LN measures relate to off-line behavioral measures of reading related skills. First, similar to our previous study (Žarić et al., 2014), at T1 we regressed the latency of the MMN in the AV0 condition with composite behavioral scores, including reading fluency and accuracy as well as letter-speech sound coupling accuracy and speed (Fig. 3-4). Second, we investigated the predictive value of the MMN latency in the AV0 condition on later behavioral performance. And finally, we asessed the relation between behavioral performance and LN latency (Fig. 3-5). We only report FDR corrected significance values $(\mathrm{q}<.05)$.
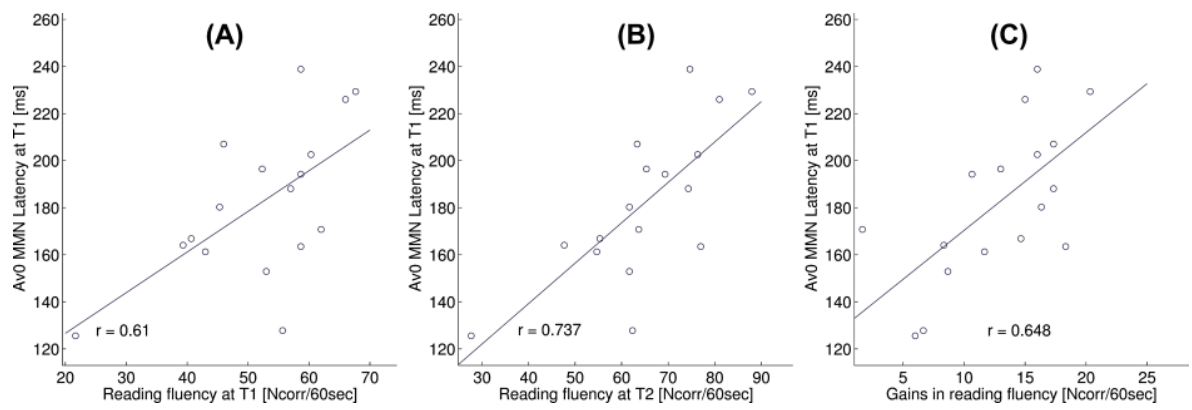

Figure 3-4. Relation between the $M M N$ latency [ms] in the simultaneous crossmodal condition (AV0) at $\mathrm{T} 1$ and: (A) reading fluency (composite score including: 3DM, EMT, "De kat") [Ncorr. words/60s] at T1; (B) reading fluency at T2; (C) gains in reading fluency from $\mathrm{T} 1$ to $\mathrm{T} 2$.

As for the correlations between MMN latency in the AV0 condition and behavioral scores at T1, also in the current sample of dyslexics MMN latency significantly correlated with individual differences in word reading fluency $(r=.610 ; p=.009 ;$ Fig. 3-4). Moreover, the latency of the MMN in the AV0 condition at $\mathrm{T} 1$ was predictive of word reading fluency at $\mathrm{T} 2(\mathrm{r}=.737, \mathrm{p}=.001)$ and of gains in word reading fluency from $\mathrm{T} 1$ to $\mathrm{T} 2(\mathrm{r}=.648, \mathrm{p}=.005)$. Furthermore, MMN latency at T1 was a significant predictor of reading fluency 
at T2 even when the autoregressive effects of reading fluency at T1 were removed. Thus, linear regression including both MMN latency and reading fluency at T1 as predictors yielded a significant overall effect $(r=.962, \mathrm{p}<.001)$ as well as separate effects for MMN latency ( $\mathrm{r}_{\mathrm{partial}}=.603, \mathrm{p}=.013$ ) and reading fluency at $\mathrm{T} 1$ ( $\left.\mathrm{r}_{\mathrm{partial}}=.914, \mathrm{p}<.001\right)$. In all cases and in accordance with our first study, a longer MMN latency was associated with better performance.

As the crossmodal LN latency significantly changed following the training, we also tested whether this change relates to behavioral scores. AV200 LN latency at T2 regressed significantly on composite scores of letter-speech sound accuracy $(\mathrm{r}=-.844, \mathrm{p}<.001)$ at T2 (Fig. 3-5). LN latency in AV0 condition did not significantly correlate with behavioral measures after correction for multiple comparisons.

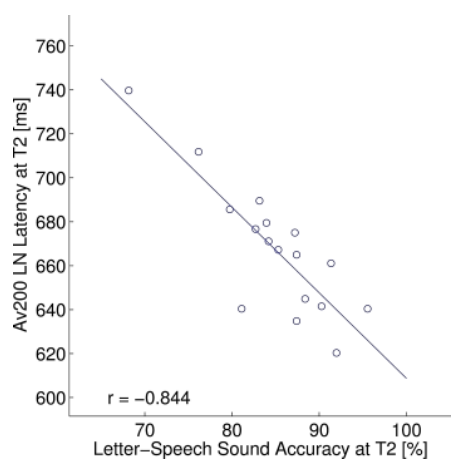

Figure 3-5. Relation between the LN latency [ms] in the asynchronous crossmodal condition (AV200) and letter-speech sound accuracy (composite score including: spelling, letter-speech sound identification and letter-speech sound discrimination accuracy) [\% correct] at T2

\section{Discussion}

The present study investigated the development of ERP measures of letterspeech sound integration and speech processing in dyslexic children over a period of 6 months during which they received both their regular $3^{\text {rd }}$ grade reading curriculum and extensive training in letter-speech sound coupling (Tijms, 2007). We were interested whether reduced crossmodal ERP responses in dyslexics show any indication of malleability and whether ERP changes 
relate to behavioral improvements. To this end we investigated crossmodal influences on vowel evoked MMN and LN responses due to the presentation of the letter ' $a$ ' together with the standard vowel sound ' $a$ ' and the deviant vowel sound 'o' (Froyen et al., 2009, 2008, 2011; Žarić et al., 2014). Our results of the first measurement (T1) demonstrate normal auditory MMN/LN change detection responses to spoken vowels /a/ and /o/ together with reduced letter-speech sound integration, thereby confirming previous findings in the current subsample of dyslexic children (Žarić et al., 2014). They also confirm a significant relation between individual differences in reading fluency and the latency of the MMN in the simultaneous crossmodal condition (AV0). The present study extends these findings by showing that the AV0 MMN latency also predicts reading fluency at the second measurement (T2) and gains in reading fluency from T1 to T2. Interestingly, our results suggest moderate improvements in the neural integration of letters and speech sounds over a 6 months period including schooling and training, with earlier (and enhanced) crossmodal effects in the LN window. Furthermore, earlier LN latency at T2 correlated significantly with higher behavioral accuracy in letter-speech sound coupling.

Auditory deviancy elicited significant MMN and LN responses with a typical fronto-central topographical distribution at both measurements, confirming typical MMN change detection responses to the vowels in children with developmental dyslexia (Czamara et al., 2011; Froyen et al., 2011; Neuhoff et al., 2012; Roeske et al., 2011; Žarić et al., 2014). In fact, our crossmodal paradigm included vowels as this allows the investigation of letter-speech sound integration deficits beyond possible speech processing deficits that have been found using more subtle speech changes involving, for example stop consonants (e.g. Csépe, 2003; Schulte-Körne et al., 1998). Correspondingly, the vowel-evoked MMN and LN responses did not change significantly following the training. This is in concordance with the results of a behavioral study which showed that pre-reading children at familial risk for dyslexia may exhibit problems in learning letter-sound associations during a training without phonological deficits before or after the training (Blomert \& Willems, 2010). 
As for the general pattern of auditory evoked responses, the relatively small N1-P2 complex in the auditory as compared to the AV0 condition is in line with previous findings using the same paradigm in typical readers of different age. That is, a comparably small N1-P2 response was observed in the auditory condition in 8-year old children, with a more pronounced response in 11-year old children (Froyen et al., 2009) and a clear response in adults (Froyen et al., 2008). Furthermore, in developmental ERP studies using e.g. click sounds or speech sounds it is often not possible to measure a clear N1 and/or P2 response in children younger than 10 years of age (see e.g. Ponton et al., 2000; Maurer et al., 2003a; Bonte et al., 2007). Although this would need to be addressed in future research, one may speculate that the clear N1-P2 response elicited by standard and deviant stimuli in the AV0 condition is due to an enhancement of auditory responses when accompanied by simultaneous visual stimuli (see e.g. Hyde et al., 2010; Fort et al., 2002a, 2002b; Giard and Peronnet, 1999).

\subsection{Reduced LN letter effects and changes in its neural timing}

In 9-year old normally reading children we previously observed enhanced MMN and LN responses to both synchronous and asynchronous letter speech sound pairs (Žarić et al., 2014). Similar to the larger sample of 36 9-year old dyslexic children that participated at T1 (Žarić et al., 2014), the present subsample of 17 dyslexic children that participated both at T1 and T2, initially showed no LN enhancement due to the presentation of the letter, confirming weak neural integration of letters and speech sounds (Blau et al., 2010; Žarić et al., 2014). The children did show a crossmodal MMN enhancement, although, like in the most severely dysfluent dyslexics of our previous study (Žarić et al., 2014), this effect only reached significance in the AV200 condition. A reduced late negativity in response to the deviant speech syllable / ba/ relative to the standard syllable /da/, in a broader time window (300-700 ms) overlapping with our crossmodal LN (600-750 ms), has been proposed as a potential endophenotype for dyslexia (Czamara et al., 2011; Neuhoff et al., 2012; Roeske et al., 2011). Although the functional role of the $\mathrm{LN}$ is not yet well understood, with respect to more automatic, perceptually driven letter-speech sound 
integration in the MMN window, the crossmodal LN enhancement may reflect more cognitive, associative and/or attentional aspects of this integration. In particular, multiple studies using different paradigms have indicated a late sensitivity to orthographic-phonological interactions in children (Froyen et al., 2009; Hasko et al., 2013; Jost, Eberhard-Moscicka, Frisch, Dellwo, \& Maurer, 2013; Žarić et al., 2014) and adults (Lafontaine et al., 2012; Pattamadilok et al., 2011; Savill \& Thierry, 2011). While these late orthographic-phonological interactions are typically observed during demanding meta-phonological tasks in adults (Lafontaine et al., 2012; Pattamadilok et al., 2011), in children they may also occur during the integration of simple letter-vowel pairs (Froyen et al., 2009; Žarić et al., 2014), and integration of audiovisual words (Jost et al., 2013). These late effects were found to be disrupted in dyslexic children (Froyen et al., 2011; Hasko et al., 2013; Žarić et al., 2014) and adults (Savill \& Thierry, 2011). Together with these previous findings, the observed reduced crossmodal LN effect may thus suggest a diminished capability to access and/or manipulate letter-speech sound representations (Blau et al., 2010; Ramus \& Ahissar, 2012; Savill \& Thierry, 2011), or impairments in attentionally-mediated integration (Czamara et al., 2011; Neuhoff et al., 2012; Savill \& Thierry, 2011, 2012) in dyslexic children. It still remains an open question whether this impaired letter-speech sound integration is a specific deficit, or whether it stems from a more fundamental impairment in audiovisual integration (Hahn, Foxe, \& Molholm, 2014).

Whereas in both crossmodal conditions, the LN effect did reach significance at the second measurement, the size of this effect did not change significantly from T1 to T2. Significant changes in letter-speech sound integration were only observed in the neural timing of this effect. That is, LN latency in the crossmodal conditions did show a significant decrease from T1 to T2. The behavioral relevance of this change is further indicated by the observation that earlier LN latency at T2 significantly related to more accurate behavioral coupling of letters and speech sounds. This earlier late negativity at T2 may thus be a sign of an improvement in the capability to access and/or manipulate the crossmodal representations (Blau et al., 2010; Ramus \& Ahissar, 2012; 
Savill \& Thierry, 2011). In future studies, it would be interesting to test whether these type of ERP latency changes relate to observed increases in white matter connectivity following behavioral intervention in 8-10 year old poor readers (Keller \& Just, 2009). Although the crossmodal LN latency related to behavioral letter-speech sound coupling at T2, we measured only one group of children that all followed the same training, which makes it difficult to fully disentangle specific training effects and more general maturational changes. On the other hand, if the shortening of crossmodal LN latency were fully determined by maturational changes in ERP morphology and/or timing, we would expect similar changes in the auditory only condition, which was not the case (Martin, Shafer, Morr, Kreuzer, \& Kurtzberg, 2003; Maurer, Bucher, Brem, \& Brandeis, 2003b; Shafer, Morr, Kreuzer, \& Kurtzberg, 2000). Furthermore, although latency measures of sustained ERP responses such as the LN may show larger intersubject variability as compared to shorter-lasting responses such as the MMN, this appears not to be the case in the present data as the range of individual participant's peak latencies was comparable across the MMN and LN responses (for example see Figs. 4 and 5). Together, the present findings suggest moderate improvement in the neural integration of letters and speech sounds due to a combination of intensive training together with the regular curriculum, especially in the later (timing) aspects of this integration.

\subsection{Reading fluency and the timing of the crossmodal MMN}

In addition to a potentially malleable late integration of letters and speech sounds, our results also suggest a less flexible early integration that may form a bottleneck in developing reading fluency. As in our previous study (Žarić et al., 2014), in the present subsample of 9-year-old dyslexic children, better reading fluency significantly correlated with a longer latency of the MMN in the simultaneous crossmodal condition (AV0). In particular, AV0 MMN latency not only correlated with reading fluency at $\mathrm{T} 1$, but also with reading fluency at $\mathrm{T} 2$ and with fluency gains from T1 to T2. Based on a further analysis of the underlying N1 and P2 ERP components, our previous results showed that this was due to a shorter-lasting, reduced MMN response, encompassing only the 
N1 window in a group of most severely dysfluent dyslexics as compared to a longer lasting MMN response, encompassing both the N1 and P2 windows in moderately dysfluent dyslexic and typical readers (Žarić et al., 2014). As ten of the current 17 children belonged to the severely dysfluent group, at the group level our results at T1 showed a shorter-lasting, reduced mismatch response in the AV0 condition, with a significant crossmodal enhancement in the N1 but not the P2 window (and no significant crossmodal MMN enhancement). Although little is known on the specific functional processes underlying the $\mathrm{P} 2$, recent evidence indicates that this deflection is specifically sensitive to the audiovisual integration of orthographic and phonological units (Pattamadilok et al., 2011) and of visual articulatory gestures and speech (Baart, Stekelenburg, \& Vroomen, 2014; Knowland, Mercure, Karmiloff-Smith, Dick, \& Thomas, 2014; Pilling, 2009; van Wassenhove, Grant, \& Poeppel, 2005). The N1 window may thus reflect a first step of audiovisual convergence, independently of reading fluency, which, especially in the most severely dysfluent dyslexics is followed by a reduced speech specific integration of letters and speech sounds in the P2 window (Žarić et al., 2014).

The absence of a significant crossmodal MMN in the synchronous condition may represent difficulties in forming a clear letter-speech sound representation (Blomert, 2011), like 'graphonemes' (Whitney \& Cornelissen, 2005). This more basic impairment, usually associated with severe cases (Roeske et al., 2011; Žarić et al., 2014), could be more resistant to change, than later more cognitive processes. In agreement with this suggestion, neither the latency nor the amplitude of the MMN letter effect showed a significant change from T1 to T2. Moreover, the children with later AV0 MMN latency (i.e. longer lasting MMN responses) at the time of the first measurement (1) read more fluently at the beginning, (2) stayed more fluent at second measurement, and (3) gained more from literacy instruction and training. Although further studies are needed, the timing of this type of crossmodal change detection responses may provide a biomarker that could contribute to a better prediction of reading gains and/or individual tailoring of dyslexia training/intervention strategies (Leppänen, 2013). 


\subsection{Conclusion}

Learning to read and its first step of mapping letters to speech sounds influences cortical networks underlying visual, auditory and higher-order language functions (Brem et al., 2010; Dehaene et al., 2010). Failure to successfully map letter-speech sound correspondences may be a proximate cause of reading failure (Blau et al., 2010; Blomert, 2011) and is related to an attenuated brain activation in dyslexia (Blau et al., 2010; Froyen et al., 2011; Žarić et al., 2014). The present findings suggest that the reduced neural integration of letters and speech sounds in dyslexic children may show moderate improvement over a period with reading instruction and letterspeech sound training, particularly in the timing of later aspects of this integration ( $\mathrm{LN}$ window). Our findings additionally point to a less flexible early integration (MMN window), with individual differences in its timing predicting gains in reading fluency. Further research should examine these improvements on a longer time scale as it is a 'long road' to fully automatized letter-speech sound integration (Froyen et al., 2009, 2008). Moreover, it would be important to further differentiate effects specific to intensive training in letter-speech sound coupling and more general maturational effects, by additionally following longitudinal trajectories of letter-speech sound integration in dyslexic children that do not receive this training. Together these studies will contribute to a better prediction of reading gains and/or individual tailoring of dyslexia training/intervention strategies.

\section{Acknowledgements}

Our co-author, professor Leo Blomert passed away on November 25, 2012. He contributed significantly to the project prior to his untimely decease.

This project is funded by the National Initiative Brain \& Cognition (NIHC), a division of the Netherlands Organization for Scientific Research (NWO) as a part of the research program "Fluent reading acquisition neurocognitively decomposed: the case of dyslexia (HCMI 10-59)" under grant number 056-14015. 
We are grateful to Suzanne van Grieken for her help in preparation and organization of the research, and Marlena van Langevelde, Gert-Jan Munneke, Jitka Annen, Mandy Meijer, Helene Vos and Jorinde Wesseling for their help during data collection. We would like to express our appreciation to the children that participated in the research and their parents.

\section{References}

Achenbach, T. M., \& McConaughy, S. H. (2003). The Achenbach System of Empirically Based Assessment (ASEBA). In C. R. Reynolds \& R. W. Kamphaus (Eds.), Handbook of psychological and educational assessment of children (2nd ed., pp. 406-430). New York, NY: Guilford Press.

Andres, A. J. D., Oram Cardy, J. E., \& Joanisse, M. F. (2011). Congruency of auditory sounds and visual letters modulates mismatch negativity and P300 event-related potentials. International Journal of Psychophysiology: Official Journal of the International Organization of Psychophysiology, 79(2), 137-46. doi:10.1016/j.ijpsycho.2010.09.012

Annett, M. (1979). Family handedness in three generations predicted by the right shift theory. Annals of Human Genetics, 42(4), 479-491. doi:10.1111/j.1469-1809.1979.tb00681.x

Aravena, S., Snellings, P., Tijms, J., \& van der Molen, M. W. W. (2013). A lab-controlled simulation of a letter-speech sound binding deficit in dyslexia. Journal of Experimental Cbild Psychology, 115(4), 691-707. doi:10.1016/j.jecp.2013.03.009

Baart, M., Stekelenburg, J. J., \& Vroomen, J. (2014). Electrophysiological evidence for speechspecific audiovisual integration. Neuropsychologia, 53, 115-21.

doi:10.1016/j.neuropsychologia.2013.11.011

Benjamini, Y., \& Hochberg, Y. (1995). Controlling the false discovery rate: a practical and powerful approach to multiple testing. Journal of the Royal Statistical Society. Series B (Methodological), 57(1), 289-300. Retrieved from http://www.jstor.org/stable/10.2307/2346101

Bishop, D. V. M. (2007). Using mismatch negativity to study central auditory processing in developmental language and literacy impairments: where are we, and where should we be going? Psychological Bulletin, 133(4), 651-72. doi:10.1037/0033-2909.133.4.651

Blau, V., Reithler, J., van Atteveldt, N., Seitz, J., Gerretsen, P., Goebel, R., \& Blomert, L. (2010). Deviant processing of letters and speech sounds as proximate cause of reading failure: a functional magnetic resonance imaging study of dyslexic children. Brain: A Journal of Neurology, 133(Pt 3), 868-79. doi:10.1093/brain/awp308

Blau, V., van Atteveldt, N., Ekkebus, M., Goebel, R., \& Blomert, L. (2009). Reduced neural integration of letters and speech sounds links phonological and reading deficits in adult dyslexia. Current Biology : CB, 19(6), 503-8. doi:10.1016/j.cub.2009.01.065

Blomert, L. (2005). Dyslexie in Nederland. Amsterdam: Uitgeverij Nieuwezijds. Amsterdam: Uitgeverij Nieuwezijds. Retrieved from http://www.boomtestuitgevers.nl/upload/Dyslexie_in_Nederland_Leo_Blomert.pdf

Blomert, L. (2011). The neural signature of orthographic-phonological binding in successful and failing reading development. NeuroImage, 57(3), 695-703.

doi:10.1016/j.neuroimage.2010.11.003 


\section{Crossmodal deficit in dyslexic children}

Blomert, L., \& Vaessen, A. A. (2009). 3DM Differential diagnostics for dyslexia: Cognitive analysis of reading and spelling. Amsterdam: Boom Test Publishers.

Blomert, L., \& Willems, G. (2010). Is there a causal link from a phonological awareness deficit to reading failure in children at familial risk for dyslexia? Dyslexia, 16(4), 300-317. doi:10.1002/dys

Boersma, P., \& Weenink, D. (2002). Praat: doing phonetics by computer (Version 4.0. 26). Amsterdam: University of Amsterdam. Retrieved from http://www.fon.hum.uva.nl/praat.

Bonte, M. L., \& Blomert, L. (2004). Developmental dyslexia: ERP correlates of anomalous phonological processing during spoken word recognition. Brain Research. Cognitive Brain Research, 21(3), 360-76. doi:10.1016/j.cogbrainres.2004.06.010

Bonte, M. L., Poelmans, H., \& Blomert, L. (2007). Deviant neurophysiological responses to phonological regularities in speech in dyslexic children. Neuropsychologia, 45(7), 1427-37. doi:10.1016/j.neuropsychologia.2006.11.009

Booth, J. R., Burman, D. D., Van Santen, F. W., Harasaki, Y., Gitelman, D. R., Parrish, T. B., \& Marsel Mesulam, M. M. (2001). The development of specialized brain systems in reading and oral-language. Child Neuropsychology : A Journal on Normal and Abnormal Development in Childhood and Adolescence, 7(3), 119-41. doi:10.1076/chin.7.3.119.8740

Brem, S., Bach, S., Kucian, K., Guttorm, T. K., Martin, E., Lyytinen, H., ... Richardson, U. (2010). Brain sensitivity to print emerges when children learn letter-speech sound correspondences. Proceedings of the National Academy of Sciences of the United States of America, 107(17), 7939-44. doi:10.1073/pnas.0904402107

Brus, B. T., \& Voeten, M. J. M. (1973). Eén-minuut test, vorm A en B; Verantwoording en Handleiding. Nimegen: Berkenhout Testmateriaal.

Bus, A. G., \& Ijzendoorn, M. H. Van. (1999). Phonological Awareness and Early Reading : A Meta-Analysis of Experimental Training Studies. Journal of Educational Psychology, 91(3), 403-414. doi:10.1037/0022-0663.91.3.403

Cheour, M., Korpilahti, P., Martynova, O., \& Lang, a H. (2001). Mismatch negativity and late discriminative negativity in investigating speech perception and learning in children and infants. Audiology \& Neuro-Otology, 6(1), 2-11. doi:46804

Cheour, M., Shestakova, A., Alku, P., Ceponiene, R., \& Näätänen, R. (2002). Mismatch negativity shows that $3-6$-year-old children can learn to discriminate non-native speech sounds within two months. Neuroscience Letters, 325, 187-190.

Cheour-Luhtanen, M., Alho, K., Kujala, T., Sainio, K., Reinikainen, K., Renlund, M., ... Näätänen, R. (1995). Mismatch negativity indicates vowel discrimination in newborns. Hearing Research, 82, 53-58. Retrieved from http://www.sciencedirect.com/science/article/pii/037859559400164L

Csépe, V. (1995). On the origin and development of the mismatch negativity. Ear and Hearing, 16(1), 91-104. Retrieved from http:/ / scholar.google.com/scholar?hl=en\&btnG=Search\&q=intitle:On + the + origin + and + development + of + the + mismatch + negativity $\# 0$

Csépe, V. (2003). Auditory event-related potentials in studying developmental dyslexia. In V. Csépe (Ed.), Dyslexia: Different brain, different behavior (pp. 81-112). New York, NY: Kluwer Academic Publishers - Plenum Publishers.

Czamara, D., Bruder, J., Becker, J., Bartling, J., Hoffmann, P., Ludwig, K. U., ... Schulte-Körne, 
G. (2011). Association of a rare variant with mismatch negativity in a region between KIAA0319 and DCDC2 in dyslexia. Behavior Genetics, 41(1), 110-9. doi:10.1007/s10519010-9413-6

de Vos, T. (2007). Schoolvaardigheidstoets Technisch Lezen. Amsterdam: Boom test uitgevers.

Dehaene, S., Pegado, F., Braga, L. W., Ventura, P., Nunes Filho, G., Jobert, A., ... Cohen, L. (2010). How learning to read changes the cortical networks for vision and language. Science (New York, N.Y.), 330(6009), 1359-64. doi:10.1126/science.1194140

Delorme, A., \& Makeig, S. (2004). EEGLAB: an open source toolbox for analysis of single-trial EEG dynamics including independent component analysis. Journal of Neuroscience Methods, 134, 9-21. Retrieved from http://www.sciencedirect.com/science/article/pii/S0165027003003479

Ehri, L. C. (2005). Learning to Read Words: Theory, Findings, and Issues. Scientific Studies of Reading, 9(2), 167-188. doi:10.1207/s1532799xssr0902_4

Fort, A., Delpuech, C., Pernier, J., \& Giard, M. H. (2002a). Early auditory-visual interactions in human cortex during nonredundant target identification. Cognitive Brain Research, 14(1), 20-30. doi:10.1016/S0926-6410(02)00058-7

Fort, A., Delpuech, C., Pernier, J., \& Giard, M.-H. (2002b). Dynamics of cortico-subcortical cross-modal operations involved in audio-visual object detection in humans. Cerebral Cortex (New York, N.Y. : 1991), 12(10), 1031-1039. doi:10.1093/cercor/12.10.1031

Froyen, D., Bonte, M. L., van Atteveldt, N., \& Blomert, L. (2009). The long road to automation: neurocognitive development of letter-speech sound processing. Journal of Cognitive Neuroscience, 21(3), 567-80. doi:10.1162/jocn.2009.21061

Froyen, D., Van Atteveldt, N., Bonte, M., \& Blomert, L. (2008). Cross-modal enhancement of the MMN to speech-sounds indicates early and automatic integration of letters and speech-sounds. Neuroscience Letters, 430(1), 23-8. doi:10.1016/j.neulet.2007.10.014

Froyen, D., Willems, G., \& Blomert, L. (2011). Evidence for a specific cross-modal association deficit in dyslexia: an electrophysiological study of letter-speech sound processing. Developmental Science, 14(4), 635-48. doi:10.1111/j.1467-7687.2010.01007.x

Giard, M. H., \& Peronnet, F. (1999). Auditory-visual integration during multimodal object recognition in humans: a behavioral and electrophysiological study. Journal of Cognitive Neuroscience, 11(5), 473-490. doi:10.1162/089892999563544

Hahn, N., Foxe, J. J., \& Molholm, S. (2014). Impairments of multisensory integration and crosssensory learning as pathways to dyslexia. Neuroscience \& Biobehavioral Reviews, 47, 384-392. doi:10.1016/j.neubiorev.2014.09.007

Hasko, S., Bruder, J., Bartling, J., \& Schulte-Körne, G. (2012). N300 indexes deficient integration of orthographic and phonological representations in children with dyslexia. Neuropsychologia, 50(5), 640-54. doi:10.1016/j.neuropsychologia.2012.01.001

Hasko, S., Groth, K., Bruder, J., Bartling, J., \& Schulte-Körne, G. (2013). The time course of reading processes in children with and without dyslexia: an ERP study. Frontiers in Human Neuroscience, 7(October), 570. doi:10.3389/fnhum.2013.00570

Hommet, C., Vidal, J., Roux, S., Blanc, R., Barthez, M. A., De Becque, B., ... Gomot, M. (2009). Topography of syllable change-detection electrophysiological indices in children and adults with reading disabilities. Neuropsychologia, 47(3), 761-70. doi:10.1016/j.neuropsychologia.2008.12.010

Hyde, D. C., Jones, B. L., Porter, C. L., \& Flom, R. (2010). Visual stimulation enhances auditory 


\section{Crossmodal deficit in dyslexic children}

processing in 3-month-old infants and adults. Developmental Psychobiology, 52(2), 181-189. doi:10.1002/dev.20417

Jost, L. B., Eberhard-Moscicka, A. K., Frisch, C., Dellwo, V., \& Maurer, U. (2013). Integration of Spoken and Written Words in Beginning Readers: A Topographic ERP Study. Brain Topography, 27, 786-800. doi:10.1007/s10548-013-0336-4

Jung, T. P., Makeig, S., Humphries, C., Lee, T. W., McKeown, M. J., Iragui, V., \& Sejnowski, T. J. (2000). Removing electroencephalographic artifacts by blind source separation. Psychophysiology, 37(2), 163-78. Retrieved from http://www.ncbi.nlm.nih.gov/pubmed/10731767

Kasai, K., Nakagome, K., Iwanami, A., Fukuda, M., Itoh, K., Koshida, I., \& Kato, N. (2002). No effect of gender on tonal and phonetic mismatch negativity in normal adults assessed by a high-resolution EEG recording. Brain Research. Cognitive Brain Research, 13(3), 305-12. Retrieved from http://www.ncbi.nlm.nih.gov/pubmed/11918996

Keller, T. a, \& Just, M. A. (2009). Altering cortical connectivity: remediation-induced changes in the white matter of poor readers. Neuron, 64(5), 624-31. doi:10.1016/j.neuron.2009.10.018

Knowland, V. C. P., Mercure, E., Karmiloff-Smith, A., Dick, F., \& Thomas, M. S. C. (2014). Audio-visual speech perception: a developmental ERP investigation. Developmental Science, 17(1), 110-24. doi:10.1111/desc.12098

Kujala, T., Lovio, R., Lepistö, T., Laasonen, M., \& Näätänen, R. (2006). Evaluation of multiattribute auditory discrimination in dyslexia with the mismatch negativity. Clinical Neurophysiology: Official Journal of the International Federation of Clinical Neurophysiology, 117(4), 885-93. doi:10.1016/j.clinph.2006.01.002

Lafontaine, H., Chetail, F., Colin, C., Kolinsky, R., \& Pattamadilok, C. (2012). Role and activation time course of phonological and orthographic information during phoneme judgments. Neuropsychologia, 50(12), 2897-906. doi:10.1016/j.neuropsychologia.2012.08.020

Lakens, D. (2013). Calculating and reporting effect sizes to facilitate cumulative science: A practical primer for t-tests and ANOVAs. Frontiers in Psychology, 4(NOV), 1-12. doi:10.3389/fpsyg.2013.00863

Lee, T. W., Girolami, M., \& Sejnowski, T. J. (1999). Independent component analysis using an extended infomax algorithm for mixed subgaussian and supergaussian sources. Neural Computation, 11(2), 417-41. Retrieved from http://www.ncbi.nlm.nih.gov/pubmed/9950738

Leppänen, P. H. T. (2013). Introduction to the special issue on brain event-related potentials as biomarkers of language and literacy development, feedback, and intervention. Developmental Neuropsychology, 38(8), 507-13. doi:10.1080/87565641.2013.829842

Lohvansuu, K., Hämäläinen, J. a, Tanskanen, A., Bartling, J., Bruder, J., Honbolygó, F., ... Leppänen, P. H. T. (2013). Separating mismatch negativity (MMN) response from auditory obligatory brain responses in school-aged children. Psychophysiology, 50(7), 640-52. doi:10.1111/psyp.12048

Luck, S. J. (2005). An introduction to the event-related potential technique. Cambridge, MA: The MIT Press.

Lyon, G. R., Shaywitz, S. E., \& Shaywitz, B. A. (2003). A definition of dyslexia. Annals of Dyslexia, 53(1), 1-14. doi:10.1007/s11881-003-0001-9

Martin, B. A., Shafer, V. L., Morr, M. L., Kreuzer, J. a, \& Kurtzberg, D. (2003). Maturation of 
mismatch negativity: a scalp current density analysis. Ear and Hearing, 24, 463-471. doi:10.1097/01.AUD.0000100306.20188.0E

Maurer, U., Brem, S., Kranz, F., Bucher, K., Benz, R., Halder, P., ... Brandeis, D. (2006). Coarse neural tuning for print peaks when children learn to read. NeuroImage, 33(2), 749-58. doi:10.1016/j.neuroimage.2006.06.025

Maurer, U., Bucher, K., Brem, S., \& Brandeis, D. (2003a). Altered responses to tone and phoneme mismatch in kindergartners at familial dyslexia risk. Neuroreport, 14(17), 2245-50. doi:10.1097/01.wnr.0000096518.69073.a7

Maurer, U., Bucher, K., Brem, S., \& Brandeis, D. (2003b). Development of the automatic mismatch response: From frontal positivity in kindergarten children to the mismatch negativity. Clinical Neurophysiology, 114, 808-817. doi:10.1016/S1388-2457(03)00032-4

McNorgan, C., Randazzo-Wagner, M., \& Booth, J. R. (2013). Cross-modal integration in the brain is related to phonological awareness only in typical readers, not in those with reading difficulty. Frontiers in Human Neuroscience, 7(July), 388. doi:10.3389/fnhum.2013.00388

Mittag, M., Alho, K., Takegata, R., Makkonen, T., \& Kujala, T. (2013). Audiovisual attention boosts letter-speech sound integration. Psychophysiology, 50, 1034-1044. doi:10.1111/psyp.12085

Mittag, M., Takegata, R., \& Kujala, T. (2011). The effects of visual material and temporal synchrony on the processing of letters and speech sounds. Experimental Brain Research. Experimentelle Hirnforscbung. Expérimentation Cérébrale, 211(2), 287-98. doi:10.1007/s00221011-2686-z

Näätänen, R. (2001). The perception of speech sounds by the human brain as reflected by the mismatch negativity (MMN) and its magnetic equivalent (MMNm). Psychophysiology, 38(1), $1-21$.

Näätänen, R., Paavilainen, P., Rinne, T., \& Alho, K. (2007). The mismatch negativity (MMN) in basic research of central auditory processing: a review. Clinical Neurophysiology : Official Journal of the International Federation of Clinical Neurophysiology, 118(12), 2544-90. doi:10.1016/j.clinph.2007.04.026

Neuhoff, N., Bruder, J., Bartling, J., Warnke, A., Remschmidt, H., Müller-Myhsok, B., \& SchulteKörne, G. (2012). Evidence for the late MMN as a neurophysiological endophenotype for dyslexia. PloS One, 7(5), e34909. doi:10.1371/journal.pone.0034909

Pattamadilok, C., Perre, L., \& Ziegler, J. C. (2011). Beyond rhyme or reason: ERPs reveal taskspecific activation of orthography on spoken language. Brain and Language, 116(3), 116-24. doi:10.1016/j.bandl.2010.12.002

Pilling, M. (2009). Auditory event-related potentials (ERPs) in audiovisual speech perception. Journal of Speech, Language, and Hearing Research : JSLHR, 52(4), 1073-81. doi:10.1044/10924388(2009/07-0276)

Ponton, C. W., Eggermont, J. J., Kwong, B., \& Don, M. (2000). Maturation of human central auditory system activity: evidence from multi-channel evoked potentials. Clinical Neurophysiology: Official Journal of the International Federation of Clinical Neurophysiology, 111(2), 220-36. Retrieved from http://www.ncbi.nlm.nih.gov/pubmed/10680557

Ramus, F., \& Ahissar, M. (2012). Developmental dyslexia: the difficulties of interpreting poor performance, and the importance of normal performance. Cognitive Neuropsychology, 29(12), 104-22. doi:10.1080/02643294.2012.677420 


\section{Crossmodal deficit in dyslexic children}

Ramus, F., \& Szenkovits, G. (2008). What phonological deficit? Quarterly Journal of Experimental Psychology (2006), 61(1), 129-41. doi:10.1080/17470210701508822

Raven, J., Raven, J. C., \& Court, J. H. (1998). Coloured progressive matrices. Oxford, UK: Oxford Psychologists Press.

Roeske, D., Ludwig, K. U., Neuhoff, N., Becker, J., Bartling, J., Bruder, J., ... Schulte-Körne, G. (2011). First genome-wide association scan on neurophysiological endophenotypes points to trans-regulation effects on SLC2A3 in dyslexic children. Molecular Psychiatry, 16(1), 97107. doi:10.1038/mp.2009.102

Savill, N. J., \& Thierry, G. (2011). Reading for sound with dyslexia: evidence for early orthographic and late phonological integration deficits. Brain Research, 1385, 192-205. doi:10.1016/j.brainres.2011.02.012

Savill, N. J., \& Thierry, G. (2012). Decoding ability makes waves in reading: deficient interactions between attention and phonological analysis in developmental dyslexia. Neuropsychologia, 50(7), 1553-64. doi:10.1016/j.neuropsychologia.2012.03.008

Schulte-Körne, G., Deimel, W., Bartling, J., \& Remschmidt, H. (1998). Auditory processing and dyslexia: evidence for a specific speech processing deficit. Neuroreport, 9(2), 337-340.

Shafer, V. L., Morr, M. L., Kreuzer, J. A., \& Kurtzberg, D. (2000). Maturation of mismatch negativity in school-age children. Ear and Hearing, 21(3), 242-51. Retrieved from http:/ $/$ cat.inist.fr $/$ ?aModele $=$ afficheN\&cpsidt $=1410810$

Share, D. L. (1995). Phonological recoding and self-teaching: sine qua non of reading acquisition. Cognition, 55(2), 151-218; discussion 219-26. Retrieved from http://www.ncbi.nlm.nih.gov/pubmed/7789090

Snowling, M. J. (1998). Dyslexia as a Phonological Deficit: Evidence and Implications. Child Psychology and Psychiatry Review, 3(1), 4-11. doi:10.1017/S1360641797001366

Snowling, M. J. (2013). Early identification and interventions for dyslexia: a contemporary view. Journal of Research in Special Educational Needs, 13(1), 7-14. doi:10.1111/j.14713802.2012.01262.x

Tijms, J. (2004). A Process-Oriented Evaluation of a Computerised Treatment for Dyslexia. Educational Psychology, 24(6), 767-791. doi:10.1080/0144341042000271719

Tijms, J. (2007). The Development of Reading Accuracy and Reading Rate during Treatment of Dyslexia. Educational Psychology, 27(2), 273-294. doi:10.1080/01443410601066800

Tijms, J. (2011). Effectiveness of computer-based treatment for dyslexia in a clinical care setting: outcomes and moderators. Educational Psychology, 31(7), 873-896.

Tijms, J., \& Hoeks, J. (2005). A computerized treatment of dyslexia: benefits from treating lexico-phonological processing problems. Dyslexia (Chichester, England), 11(1), 22-40. doi:10.1002/dys.283

Vaessen, A., Bertrand, D., Tóth, D., Csépe, V., Faísca, L., Reis, A., \& Blomert, L. (2010). Cognitive development of fluent word reading does not qualitatively differ between transparent and opaque orthographies. Journal of Educational Psychology, 102(4), 827-842. doi: $10.1037 / \mathrm{a} 0019465$

van Wassenhove, V., Grant, K. W., \& Poeppel, D. (2005). Visual speech speeds up the neural processing of auditory speech. Proceedings of the National Academy of Sciences of the United States of America, 102(4), 1181-6. doi:10.1073/pnas.0408949102

Whitney, C., \& Cornelissen, P. (2005). Letter-position encoding and dyslexia. Journal of Research in 
Chapter 3

Reading, 28(3), 274-301. doi:10.1111/j.1467-9817.2005.00270.x

Wimmer, H., \& Schurz, M. (2010). Dyslexia in regular orthographies: manifestation and causation. Dyslexia, 16(4), 283-299. doi:10.1002/dys.411

Žarić, G., Fraga González, G., Tijms, J., van der Molen, M. W., Blomert, L., \& Bonte, M. (2014). Reduced neural integration of letters and speech sounds in dyslexic children scales with individual differences in reading fluency. PloS One, 9(10), e110337.

doi:10.1371/journal.pone.0110337

Ziegler, J. C., Perry, C., Ma-Wyatt, A., Ladner, D., \& Schulte-Körne, G. (2003). Developmental dyslexia in different languages: Language-specific or universal? Journal of Experimental Child Psychology, 86(3), 169-193. doi:10.1016/S0022-0965(03)00139-5 



\title{
CHAPTER 4
}

\author{
Altered patterns of \\ effective connectivity within \\ the reading network of \\ dyslexic children and \\ their relation to \\ reading dysfluency
}

Manuscript in preparation:

Žarić, G., Correia, J.M., Fraga González, G., Tijms, J., van der Molen, M., Blomert, L., Bonte, M. Altered patterns of effective connectivity within the reading network of dyslexic children and their relation to reading dysfluency. 


\section{Abstract}

Reading is a complex cognitive skill subserved by a distributed network of visual and language-related regions. Disruptions of the connectivity within this network have been associated with developmental dyslexia. To investigate whether these disruptions scale with the level of reading dysfluency, here we examined effective connectivity in the reading network of 9-year-old typically reading children $(n=20)$ and two groups of dyslexic children: severely dysfluent dyslexic $(n=17)$ and moderately dysfluent dyslexic $(n=18)$ readers. We used directed transfer function (DTF) to analyze effective connectivity in electroencephalographic (EEG) signals recorded while the children processed visual words and letter-like symbol strings. In the visual word condition, our analysis first indicated a weaker propagation from occipital to inferior-temporal channels in both dyslexic groups as compared to typical readers. Conversely, dyslexics showed stronger connectivity from left frontal to right inferiortemporal sites as well as between central and posterior sites. Interestingly, the level of reading dysfluency influenced altered connectivity patterns in dyslexics. In particular, while in moderately dysfluent dyslexics stronger connectivity was observed from central to bilateral inferior-temporal sites, the severely dysfluent dyslexic group relied more on connectivity to the right hemisphere. In the symbol string condition, differences between typical and moderately dysfluent readers were strongly diminished, whereas both groups differed substantially from the severely dysfluent readers. Our results confirm dysfunctional connectivity patterns in the reading network of dyslexic children with reduced posterior to anterior and enhanced anterior to posterior connectivity. The additional differentiation between connectivity patterns in the two dyslexic groups emphasizes the importance of considering the severity of reading dysfunction of individual dyslexic children next to group differences between typical and dyslexic readers. 


\section{Introduction}

Although reading is a complex cognitive function, 90-95\% of children master it without notable problems. Conversely, children suffering from developmental dyslexia never attain mastery level reading skills regardless of their normal cognitive abilities and educational opportunities (Blomert, 2005; Lyon, Shaywitz, \& Shaywitz, 2003). Among reading related deficits of developmental dyslexia, reading dysfluency poses the most pronounced and long lasting hurdle (Shaywitz, Morris, \& Shaywitz, 2008), especially in more transparent orthographies, such as Dutch or German (Wimmer \& Schurz, 2010). At the same time, dyslexics may show substantial inter-individual variability in their level of reading (dys)fluency (Katzir, Kim, Wolf, Morris, \& Lovett, 2008; Leinonen et al., 2001; Žarić et al., 2014). Comparisons of typical and dyslexic readers suggest abnormal functional and anatomical connectivity within the brain's reading network in dyslexia (Geschwind, 1965; Paulesu et al., 1996; Wimmer \& Schurz, 2010). Whether this atypical neural connectivity scales with differences in the level of reading (dys)fluency in dyslexic children remains an open question that is investigated in the present study.

As a complex cognitive function, reading depends on connectivity within large scale neuronal networks combining information of multiple localized processes (Engel, Fries, \& Singer, 2001). Because reading is a recent addition to the human ability ( $\sim 5000$ years old), it is subserved by brain networks originally developed for language and visual processing, rather than an evolutionarily created specific network (Hannagan, Amedi, Cohen, Dehaene-lambertz, \& Dehaene, 2015; McCandliss, Cohen, \& Dehaene, 2003). The reading network has been proposed to include three main hubs: (1) a left ventral occipitotemporal region involved in fast visual word recognition (Jobard, Crivello, \& Tzourio-Mazoyer, 2003; McCandliss et al., 2003), (2) left posterior temporal and dorsal parieto-temporal areas involved in phonological processing (Pugh et al., 2001) and multisensory grapheme-phoneme integration (Blomert, 2011; Simos et al., 2002; van Atteveldt \& Ansari, 2014), and (3) left inferior-frontal regions related to speech production and phonological recoding of words (Sandak, Mencl, Frost, \& Pugh, 2004). 
Evidence from anatomical and functional magnetic resonance imaging (MRI/fMRI) and diffusion tensor imaging (DTI) studies in dyslexia points to several possible anomalies in the distributed reading network (Blomert, 2011; McCandliss \& Noble, 2003; Richlan, Kronbichler, \& Wimmer, 2009). Investigations of morphological measures have suggested reduced gray matter in parieto-temporal and occipito-temporal areas (Linkersdörfer, Lonnemann, Lindberg, Hasselhorn, \& Fiebach, 2012; Richlan, Kronbichler, \& Wimmer, 2013) as well as abnormalities in white matter connections between temporoparietal and frontal areas in dyslexics (Vandermosten, Boets, Wouters, \& Ghesquière, 2012). Dyslexics also show reduction in functional connectivity as compared to typical readers, both during reading tasks and resting state paradigms (Finn et al., 2014; Schurz et al., 2014; van der Mark et al., 2011; Wolf et al., 2010). Increases of connectivity in dyslexic readers have also been observed and may reflect compensatory mechanisms, for example an increased recruitment of right prefrontal regions (Hoeft et al., 2007).

Electro- and magneto-encephalographic (EEG and MEG) studies investigating connectivity within the reading network have yielded a mixed pattern of results spanning brain oscillations in a large range of frequency bands. One source of variability involves the different measures of connectivity used and various tasks employed. There are several methods to determine EEG-based functional (non-directional) or effective (directional) connectivity. One of the first proposed measures of connectivity was coherence, which can be described as a correlation in the frequency domain between two channels (Varela, Lachaux, Rodriguez, \& Martinerie, 2001). However, coherence may be influenced by volume conduction and reference electrodes (Schlögl \& Supp, 2006). To overcome these problems, researchers have proposed methods based on multivariate autoregressive models and Granger causality (Granger, 1969), such as directed transfer function (DTF) and partial-directed coherence (PDC). DTF computes the ratio between the input from a given channel $\mathrm{A}$ to a given channel B and all the inputs to channel B (Blinowska, Kuś, \& Kamiński, 2004). PDC computes the ratio between the output from a given channel $A$ to a given channel B and all the outputs from channel A (Baccalá \& Sameshima, 2001). 
An MEG study investigating effective connectivity while typical adult readers processed words presented in a rapid serial visual presentation (RSVP) paradigm with connected text, found alpha band $(\alpha, 8-14 \mathrm{~Hz})$ long-band communication within the left lateralized reading network in 8 of 9 subjects, while 4 subjects also showed beta band ( $\beta, 15-30 \mathrm{~Hz}$ ) (Kujala et al., 2007). In particular, results showed activation propagation from posterior to anterior areas with inferior occipito-temporal cortex and cerebellum as the main driving nodes. In frontal regions, the information propagated from motor cortex to prefrontal and orbital frontal cortex, and from insula to the temporal lobe (Kujala et al., 2007). A more recent MEG study found an interplay of stronger feedforward and feedback connectivity for words than for false font strings between source dipoles in the left hemisphere reading network, i.e. in occipital, ventral occipito-temporal and frontal regions (Woodhead et al., 2014). A further illustration of connectivity within the typical reading network comes from an intracranial EEG study that showed an increase of activity in a broad gamma range $(\gamma, 40-150 \mathrm{~Hz})$ during sentence reading together with task dependent long distance amplitude correlations when comparing semantic, phonological and visual decision tasks (Vidal et al., 2012).

Studies examining spectral power and coherence measures in dyslexic and typically reading children at rest observed increased power in lower frequency bands in frontal and right temporal regions in dyslexics together with increased EEG coherence in the frontal, central and temporal regions for all frequency bands (Arns, Peters, Breteler, \& Verhoeven, 2007), or a mixture of increased and decreased power in the lower bands, together with stronger intra- and reduced inter-hemispheric coherence (Leisman, 2002). Due to the dynamic nature of functional brain development, patterns of typical and dysfunctional functional connectivity may also change with age (e.g. Marosi et al., 1995). Studies investigating network configuration at rest by means of graph theory found a decreased local efficiency between the sensors covering the left temporo-parietal region and the remaining sensors in the 20-29 $\mathrm{Hz}$ band and a decreased global efficiency across all frequency bands (Dimitriadis et al., 2013) or a less integrated network configuration within the $\theta$ band (Fraga González et 
al., submitted) in dyslexic children. Thus, studies at rest have reported group differences between dyslexics and normal readers in terms of coherence and power in $\delta(1-4 \mathrm{~Hz}), \theta(4-7 \mathrm{~Hz}), \alpha$ and $\beta$ bands.

EEG studies investigating task related functional connectivity have yielded different recruitment of the reading network in dyslexic and typically reading adults and children across multiple frequency bands. An EEG study employing a visual lexical-decision task to investigate differences between two subgroups of dyslexics based on their dominant type of reading difficulty found that dyslexics that had difficulty sounding out words had higher $\beta$ power in anterior relative to posterior regions, while this was reversed in dyslexics that had difficulty with memory and perception of whole-word configurations (Milne, Hamm, Kirk, \& Corballis, 2003). An EEG-fMRI study using identity judgements of visually presented stimulus pairs found that typically reading children exhibited an earlier pattern of word specific connectivity than dyslexics, while both groups had richest pseudoword specific connectivity in a later time window (Ligges, Ungureanu, Ligges, Blanz, \& Witte, 2010). Furthermore, typically reading children employed the expected left-dominant reading network, while dyslexics showed different interhemispheric connectivity pointing to the usage of the right homologue areas (Ligges et al., 2010). Another EEG study found differences in $\theta$ band during silent reading of a short story, in which typically reading children had complex inter- and intrahemispheric connectivity between close and distant brain regions, while dyslexic children showed a simpler centro-parietal configuration (Stokić, Milosavljević, \& Subotić, 2011). In summary, previous studies indicate altered functional and effective connectivity in dyslexic adults and children with a mixed pattern of results including general tendencies such as altered posterior to frontal connectivity and a relatively stronger reliance on right as compared to left hemispheric cortical regions during reading in dyslexia. Next to the use of a variety of task or rest conditions and analysis methods, across study differences may relate to differences in the level of reading dysfluency and associated use of compensatory mechanisms in dyslexics (Žarić et al., 2014, 2015). Thus, next to comparing typical versus dyslexic readers, a central aim of the present study is 
to investigate whether and how effective connectivity during reading scales with the level of dysfluency in dyslexic children

In the current EEG study we investigate patterns of effective connectivity in the reading network in typically reading children $(n=20)$ and two groups of dyslexic children: moderately dysfluent dyslexic $(n=18)$ and severely dysfluent dyslexic $(n=17)$ readers. The same groups of children were previously shown to exhibit different patterns of neural integration of letters and speech sounds (Žarić et al., 2014). In particular, neural integration of letters and speech sounds scaled with reading fluency with moderately dysfluent dyslexics showing early integration effects similar to typical readers, and a lack of late integration effects similar to severely dysfluent dyslexics (Žarić et al., 2014). We employ a visual word recognition paradigm in which children are presented with visual words and false font symbol strings in separate blocks while performing a one-back task on stimulus repetition (Fraga González et al., 2014). We quantify functional effective connectivity by applying the DTF connectivity method that gives us not only the strength of connectivity between selected electrodes, but also the direction of the activation propagation (Kamiński \& Blinowska, 1991). Focusing on the level of (dys)fluency in dyslexia, gives us the opportunity to look at differences related to the level of dysfluency that may also have contributed to the mixed results obtained in previous functional connectivity studies.

\section{Methods}

\subsection{Participants}

Participants were 56 9-year-old children including 36 dyslexic and 20 typical readers. As the same children also participated in a previous crossmodal oddball study, we refer to the corresponding paper for a detailed description of the participants and their behavioral scores (Chapter 2; Žarić et al., 2014). Dyslexic children were divided into a group of severely dysfluent dyslexics and moderately dysfluent dyslexics based on a composite score of reading fluency (Dyslexia Differential Diagnosis - 3DM reading subtest (Blomert \& Vaessen, 2009), one-minute word reading test - EMT (Brus \& Voeten, 1973), short story 
'De kat' ('The cat') reading test (de Vos, 2007); for details see Chapter 2; Žarić et al., 2014). In comparison to this previous study, one child in the severely dysfluent group was excluded due to an incomplete dataset, resulting in the inclusion of 20 typical readers, 18 moderately dysfluent dyslexics and 17 severely dysfluent dyslexics.

\subsection{Stimuli}

Visual stimuli consisted of 80 bisyllabic Dutch words and 80 corresponding symbol strings (Fraga González et al., 2014). The words were selected in a twostep process. First a larger set of words was selected from two word lists (Ghyselinck, De Moor, \& Brysbaert, 2000; Schaerlakens, Kohnstamm, \& Lejaegere, 1999) based on an age of acquisition (AOA) of 6-years or earlier. In the second step, three independent native Dutch adult speakers rated familiarity of the selected words and only words that were rated as familiar by all 3 raters were further selected for the experiment. Half of the resulting 80 words had 4-5 letters (short words) and the other half had 6-7 letters (long words). We used bold lower-case "Arial" font, size 40, for the presentation of the words. The 80 words were then converted to 80 corresponding symbol strings using a special "3elementSymbols-1600" false font (P.L. Cornelissen, personal communication October 2011). We used this false font (Fig. 4-1) as it consists of meaningless characters comparable to the Latin alphabet letters in spatial frequency, contrast characteristics and the number of line elements (Pammer et al, 2004). Words and symbols were presented in white on a black background at the center of the screen, covering on average $6.4^{\circ}(\mathrm{w}) \times 1.5^{\circ}(\mathrm{h})$ of the visual angle.

\begin{tabular}{|c|c|c|c|c|c|c|c|c|c|c|c|c|c|c|c|c|c|c|c|c|c|c|c|c|c|}
\hline a & $b$ & C & $d$ & e & $f$ & g & h & $i$ & $\mathrm{j}$ & $\mathrm{k}$ & I & & $n$ & 0 & $p$ & $q$ & $r$ & $S$ & $\mathrm{t}$ & $\mathrm{u}$ & V & $\mathbf{w}$ & $X$ & $y$ & Z \\
\hline$\vec{v}$ & $C$ & $\lambda_{n}$ & $C_{1}$ & e & in & $J$ & f & $u^{2}$ & $\nu$ & $u^{\prime}$ & 3 & 3 & つ & s] & $\Omega$ & $\Omega$ & $u^{\prime}$ & $x$ & $x$ & $x$ & $\delta$ & $\zeta$ & $\lambda$ & + & 困 \\
\hline
\end{tabular}

Figure 4-1. False font used in the experiment. 


\subsection{Experimental design}

Stimuli were presented in a $2 \times 2$ blocked design with separate blocks per condition type and stimulus length (Fraga González et al., 2014). In total, 4 word- and 4 symbol-blocks were presented in a pseudo-random order. Each block consisted of 44 stimulus trials. Stimuli were presented for $700 \mathrm{~ms}$ followed by a white fixation cross presented during the $1350 \mathrm{~ms}$ inter-stimulus interval (ISI). To keep participants attentive throughout the experimental session, participants were asked to performed a one-back task on stimulus repetition (Fig. 4-2). Each block contained four target trials, presented pseudorandomly, avoiding consecutive presentations of targets. Participants responded to the target trials by pressing any of the two buttons placed at the arms of the chair they were seated. Target trials were excluded from the EEG analysis. The total recording time of the experiment was 12 minutes excluding breaks in between block given to the participants as necessary. The experiment was part of a longer experimental session that included a crossmodal oddball paradigm (̌̌arić et al., 2014).

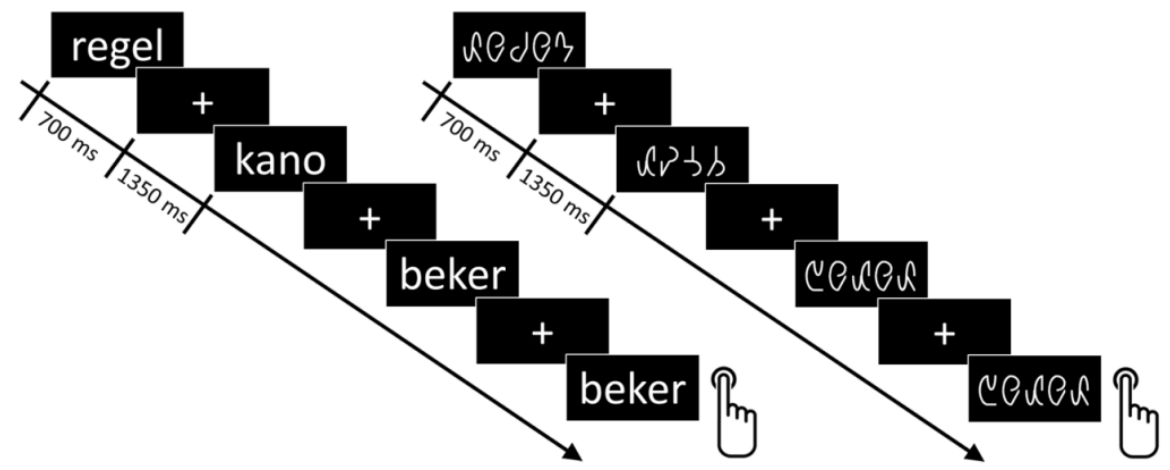

Figure 4-2. Design of the experiment.

\subsection{Behavioral data analysis}

We calculated percentages of correct target detections, average reaction times and false alarms per experimental condition to test whether the participants followed the task as intended and to evaluate possible behavioral differences between groups and conditions. To account for the fact that accuracy data tended not to be normally distributed, condition differences were examined 
with the Wilcoxon signed-ranks test for one sample and group differences with the independent samples Mann-Whitney-U test. As reaction times were normally distributed, these were analyzed with repeated measures ANOVA within factors: type (words - false font) and length (short - long) (see also Fraga González et al., 2014). In the severely dysfluent dyslexic group we excluded from further behavioral and connectivity analysis one child that had no correct responses in any of the conditions.

\subsection{EEG data recording and preprocessing}

EEG data were recorded with the 'Biosemi Active Two system' (Biosemi, Amsterdam, Netherlands). Recordings were sampled at $1024 \mathrm{~Hz}$ with a bandwidth of DC-104 Hz. EEG was measured at 64 scalp electrodes using active-channels placed according to the 10-20 international system (Electro-cap International Inc.). Additionally, the cap provided locations for two more electrodes needed for recording of the DC signal in the vicinity of PO1 (CMS) and PO2 (DRL), and the CMS was used as a recording reference. Four 'FlatType Active' electrodes were used for the bipolar measurement of eyemovements, two of which were placed below and above left eye and two at the outer canthi of each eye. Two more electrodes on the left and right mastoids were used for offline re-referencing. We kept the offset band of the electrodes from $-20 \mathrm{mV}$ to $20 \mathrm{mV}$.

The data were preprocessed and analyzed using the v12.0.5b EEGLAB toolbox (Delorme \& Makeig, 2004) (http://www.sccn.ucsd.edu/eeglab), Fieldtrip (Oostenveld, Fries, Maris, \& Schoffelen, 2011), BSMART toolbox (Cui, Xu, Bressler, Ding, \& Liang, 2008) and custom Matlab scripts (MATLAB 2014a, The MathWorks, Natick, MA). Raw EEG signals were offline re-referenced to the average of the mastoids, band-pass-filtered from 0.5 to $100 \mathrm{~Hz}$, resampled to $256 \mathrm{~Hz}$ and epoched between -500 to $900 \mathrm{~ms}$ with respect to stimulus events of the non-target trials of interest for the analysis. Epochs were baseline corrected with respect to the mean signal in the pre-stimulus period (500 ms). As this time window includes the major responses to visual words/false fonts (Dambacher et al., 2012; Laszlo \& Federmeier, 2014; Ligges et al., 2010; 
Rossell, Price, \& Nobre, 2003) and visual inspection of our data revealed that a large percentage of eye-blinks happened around $1000 \mathrm{~ms}$ post-stimulus, we decided to epoch the data up to $900 \mathrm{~ms}$ post-stimulus. Epochs including signals with amplitudes higher than $\pm 150 \mathrm{mV}$ at the EOG and scalp electrodes were classified as artifactual and discarded from further analysis. The final mean (SD) number of epochs included in the analysis is given in Table 4-1. Importantly, as we were interested in functional directed connectivity, we avoided preprocessing steps such as independent component analysis (Jung et al., 2000), which allows to identify and remove components within the signals associated with stereotypical patterns of EEG artifacts, but that may affect the results of our analysis by artificially inducing shifts in the phase of the different spectral oscillations (Kaminski \& Blinowska, 2014).

\begin{tabular}{|l|cccc|}
\hline & $\begin{array}{c}\text { Short } \\
\text { words }\end{array}$ & $\begin{array}{c}\text { Long } \\
\text { words }\end{array}$ & $\begin{array}{c}\text { Short } \\
\text { symbols }\end{array}$ & $\begin{array}{c}\text { Long } \\
\text { symbols }\end{array}$ \\
\hline Typical & $62(12)$ & $63(10)$ & $66(10)$ & $67(10)$ \\
Moderate & $63(10)$ & $59(12)$ & $58(11)$ & $62(10)$ \\
Severe & $60(13)$ & $59(13)$ & $61(13)$ & $59(12)$ \\
\hline
\end{tabular}

Table 4-1. Number of retained trials, $M(S D)$, per group per condition

\subsection{EEG data analysis}

Functional connectivity analysis was performed using 9 electrodes covering left and right posterior to anterior sites (O1, O2, P7, P8, C3, Cz, C4, F5, F6). In rare cases of noisy signals at one of these electrodes, it was replaced with an adjacent electrode: in two participants $\mathrm{O} 1$ was replaced with PO3, in one participant $\mathrm{O} 2$ was replaced with PO4 and in another participant we used F7 instead of F5. The selected 9 electrodes are reported in the literature to be in the vicinity of regions involved in reading, i.e. middle occipital areas (O1 and O2), occipito-temporal regions including inferior temporal areas (P7 and P8), somatosensory and motor areas (Cz, C3, C4) and inferior/middle frontal areas (F5 and F6) (Koessler et al., 2009). Moreover, signals from auditory cortical areas are also projected to central electrodes (Mayhew, Dirckx, Niazy, Iannetti, \& Wise, 2010). 
We calculated effective connectivity between channels using the directed transfer function (DTF, Kamiński \& Blinowska, 1991). DTF was applied across single trials from 0 to $900 \mathrm{~ms}$ and for the $1-70 \mathrm{~Hz}$ frequency range for each of the four conditions separately. Coefficients of the multivariate autoregressive (MVAR) model were calculated using BSMART as implemented in Fieldtrip (Cui et al., 2008; Oostenveld et al., 2011). The order of the model was estimated based on the Bayesian Information Criterion (Schwarz, 1978) representing the best trade-off between accuracy of fit and parsimony, as implemented in the 'Granger causal connectivity analysis' (GCCA) toolbox for Matlab (Seth, 2010). The mean (SD) model order over all trials, conditions and subjects was 4 (0.3). The MVAR coefficients were then used to compute the spectral transfer matrix, which was used for the estimation of the DTF. DTF can be described as an expansion of Granger causality to an arbitrary number of signals/channels (Blinowska et al., 2004), and computes the ratio between the inflows from channel A to channel B and the inflows from all channels to the channel $\mathrm{B}$. The resulting DTF value ranges from 0 to 1 , where 1 indicates that all the inflows to channel B come exclusively from channel A. DTF is calculated as a function of frequency and is robust to constant phase disturbances and volume conduction (Kamiński \& Blinowska, 1991). DTF has several versions depending on the type of the data for which it is calculated. Examples of applications to EEG data include studies on attention and working memory, finger movements, and localization of epileptic foci (Androulidakis et al., 2008; Blinowska, Kamiński, Brzezicka, \& Kamiński, 2013; Blinowska, Kuś, Kaminski, \& Janiszewska, 2010; Brzezicka, Kamiński, Kamiński, \& Blinowska, 2011; Ewen et al., 2015; Franaszczuk, Bergey, \& Kamiński, 1994; Ginter, Blinowska, Kamiński, \& Durka, 2001; Kuś, Ginter, \& Blinowska, 2006; Schumacher, Stiris, \& Larsson, 2015).

To investigate differences between conditions in each group of participants we used Wilcoxon signed-ranks test for paired samples. To assess whether reading related connectivity is related to reading fluency we investigated group differences in the DTF values using nonparametric Wilcoxon rank-sum test for two populations of independent samples (an equivalent of Mann-Whitney-U 
test) for each of the 70 frequencies from 1-70 Hz. We used a nonparametric test because of the relatively small number of subjects and large number of frequencies that were tested, thus avoiding making assumptions about the normality of the data for every frequency (Gibbons \& Chakraborti, 2003). We only report results that were significant $(\mathrm{q}<.05)$ after FDR multiple comparisons correction (Benjamini \& Hochberg, 1995).

\section{Results}

\subsection{Behavioral performance}

The percentages of correct responses (target trials) and false alarms (non-target trials), and mean reaction times for the correct responses across groups are given in Table 4-2. As can be seen all three groups performed the task as intended as represented by high percentages of correct responses and low percentages of false alarms. Below we first report behavioral results for the visual word and false font symbol conditions and continue with effects of stimulus length in these conditions.

Behavioral measures of target detection (in total 8 targets per condition) suggest that for all three groups it was more difficult to recognize false font symbol string repetition than the repetition of words. Typical readers showed more correct responses (CR) and less false alarms (FA) for words than for symbol strings (CR: Z=-3.853, $\mathrm{p}<.001$, Mean Rank - $\mathrm{MR}_{\mathrm{W}>\mathrm{s}}=10.95$, $\mathrm{MR}_{\mathrm{W}<\mathrm{S}}=2.00 ; \quad \mathrm{MR}_{\mathrm{SW}<\mathrm{SS}}=11.79 ; \quad \mathrm{FA}: \quad \mathrm{Z}=-3.925, \quad \mathrm{p}<.001, \quad \mathrm{MR}_{\mathrm{W}>\mathrm{s}}=.00$, $\left.M_{\mathrm{W}<S}=10.50\right)$. The rmANOVA for reaction times also yielded differences between words an symbols $\left(\mathrm{F}_{(1,19)}=14.65, \mathrm{p}=.001\right)$. Moderately dysfluent dyslexics also showed more correct responses and less false alarms for words than for symbol strings (CR: $Z=-3.663, \mathrm{p}<.001, \mathrm{MR}_{\mathrm{W}>\mathrm{s}}=9.00, \mathrm{MR}_{\mathrm{W}<\mathrm{s}}=.00$; FA: $\left.\mathrm{Z}=-3.624, \mathrm{p}<.001, \mathrm{MR}_{\mathrm{W}>\mathrm{S}}=.500, \mathrm{MR}_{\mathrm{W}<\mathrm{S}}=9.00\right)$. Reaction times did not differ for words and symbols in this group. Like the other two groups, severely dysfluent dyslexics showed less false alarms for words than for symbol strings, but the number of correct responses did not differ (FA: $Z=-3.520, p<.001$, $\left.\mathrm{MR}_{\mathrm{W}>\mathrm{S}}=.00, \mathrm{MR}_{\mathrm{W}<\mathrm{S}}=8.50\right)$. Like in typical readers, reaction times were shorter for words than for symbols (Main effect of Type: $F_{(1,13)}=5.18, p=.040$ ). 


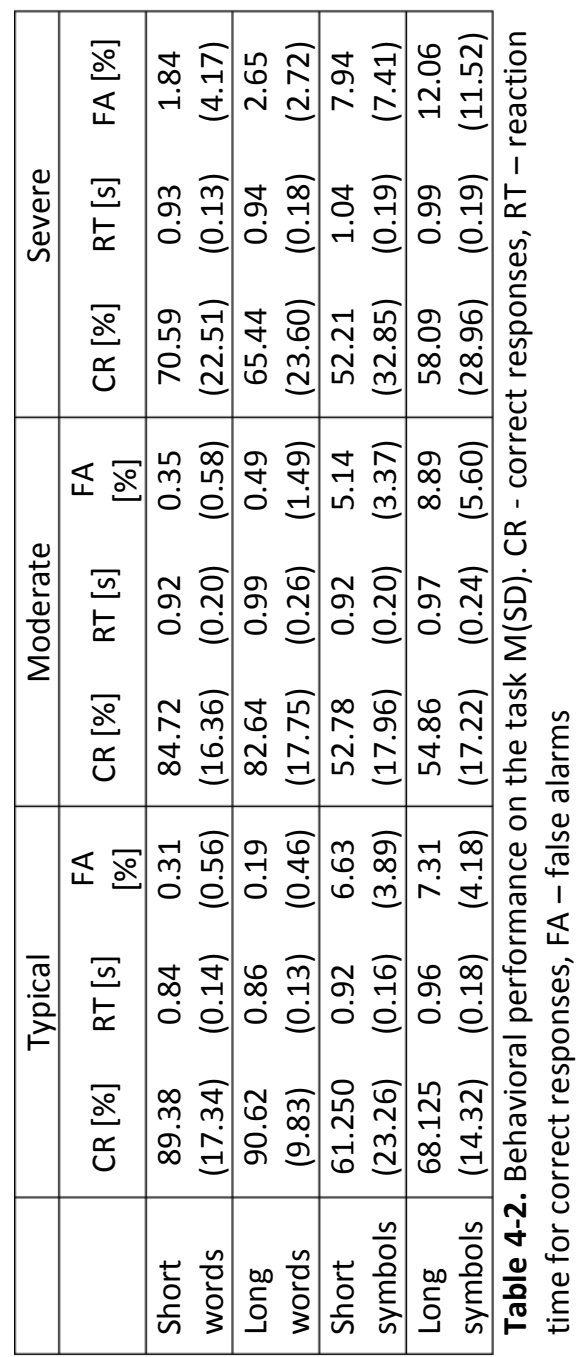


A comparison of groups showed that moderately dysfluent dyslexics were as accurate on the word repetition task as typical readers, but they were slower than the typical readers (type*group interaction $\mathrm{F}_{(1,36)}=5.33, \mathrm{p}=.027 ; \mathrm{M}(\mathrm{SD}) \mathrm{RT}$ : $\mathrm{TR}=852(118) \mathrm{ms} ; \mathrm{MDD}=956(219) \mathrm{ms})$. Furthermore, moderately dysfluent dyslexics were less accurate than typical readers in detecting targets in the false font condition $(\mathrm{U}=106.0, \mathrm{p}=.030)$. Compared to severely dysfluent dyslexics, both the typical and the moderately dysfluent dyslexic readers were more accurate in the word conditions, with no differences in reaction times. Hence, typical readers had more correct responses and less false alarms for the words (CR: $U=34.5, p<.001, F A: U=41.0, p<.001)$ than severely dysfluent dyslexics but they were not faster (there was only a main effect of type $\left(F_{(1,32)}=17.36\right.$, $\mathrm{p}<.001)$. Finally, a comparison of the two dyslexic groups yielded more correct response and less false alarms for words in moderately dysfluent dyslexic readers (CR: $\mathrm{U}=72.0, \mathrm{p}=.012, \mathrm{FA}: \mathrm{U}=46.5, \mathrm{p}<.001$ ) than in severely dysfluent dyslexics. There were no differences between the two dyslexic groups in reaction time. This pattern of results could indicate a different speed-accuracy tradeoff in the two dyslexic groups, with moderately dysfluent dyslexics performing slower but accurately in the word conditions, while severely dysfluent dyslexics do not compensate by being slower and therefore their strategy is just to 'accept' errors.

In a last analysis we tested behavioral effects of stimulus length, i.e. short versus long visual words and symbol strings. In typical readers, detection of word or symbol string repetition did not depend on stimulus length. Moderately dysfluent dyslexics however showed more false alarms and longer reaction times for long (Lo) than for short (Sh) stimuli (false alarms: $\mathrm{Z}=-2.941, \mathrm{p}=.003$, $\mathrm{MR}_{\mathrm{Sh}>\mathrm{Lo}}=4.83, \mathrm{MR}_{\mathrm{Sh}<\mathrm{Lo}}=9.89$; reaction times $\mathrm{F}_{(1,17)}=5.43, \mathrm{p}=.032$ ), while severely dysfluent dyslexics showed more false alarms for long than for short stimuli $\left(Z=-2.359, \mathrm{p}=.018, \mathrm{MR}_{\mathrm{Sh}>\mathrm{Lo}}=5.63, \mathrm{MR}_{\mathrm{Sh}<\mathrm{Lo}}=9.46\right)$. Finally, for the long stimuli, typical readers showed more correct responses than the two dyslexic groups (TR/MDD: $U=93.5, \mathrm{p}=.010$; TR/SDD: $\mathrm{U}=34.5, \mathrm{p}<.001$ ) and less false alarms than the severely dysfluent dyslexics $(\mathrm{U}=89.5, \mathrm{p}=.023)$. 


\subsection{EEG connectivity patterns for words and symbols}

We were first interested in the network of regions involved in the processing of words and symbols across the 0-900 ms period, without differentiating individual time windows. We thus concentrated our analysis on a comparison of functional effective connectivity in the EEG signals elicited by all non-target stimuli in the visual word and false font symbol string conditions, grouped across short and long stimuli.

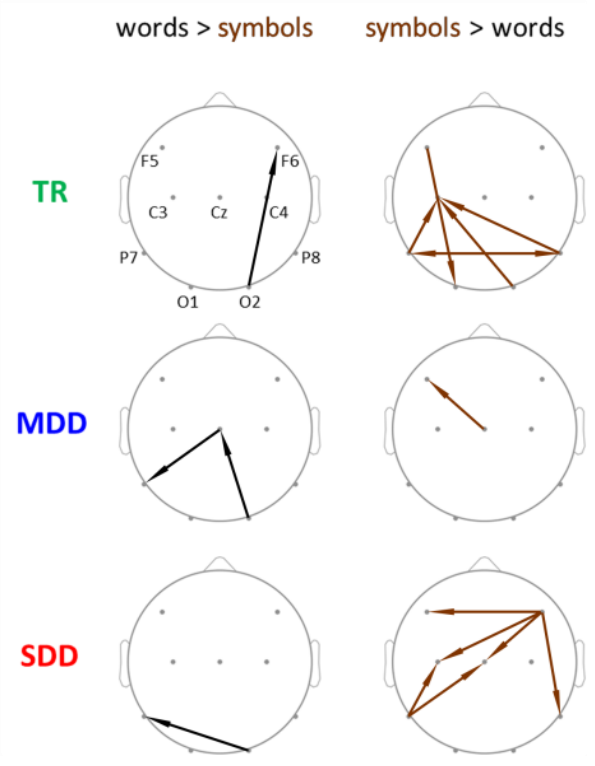

Figure 4-3. Topographic visualization of differences in connectivity between words and symbols per group. Stronger connectivity for words - black, stronger connectivity for symbols -brown. TR- typical readers, MDD - moderately dysfluent dyslexics, SDD - severely dysfluent dyslexics. Arrows - direction of the connectivity.

Figure 4-3 illustrates differences in connectivity patterns between word and false font conditions in each of the three groups. As can be seen, typical readers showed stronger right occipital to frontal connectivity for words than symbols $(\mathrm{O} 2$ to $\mathrm{F} 6,3-4 \mathrm{~Hz})$ and more widespread connectivity increases for symbols as compared to words from bilateral posterior to anterior sites (significant effects from $\mathrm{O} 2$ to $\mathrm{C} 3: 28-51 \mathrm{~Hz}, \mathrm{P} 7$ to $\mathrm{C} 3: 12-37 \mathrm{~Hz}, \mathrm{P} 8$ to $\mathrm{C} 3: 26-46 \mathrm{~Hz}$ ), as well as 
bidirectional connectivity between P7 and P8 (P7-P8: 1-37Hz, P8-P7: 16-48Hz) and anterior to posterior connectivity from F5 to O1 $(36-60 \mathrm{~Hz})$. In moderately dysfluent dyslexics, words elicited stronger connectivity from right occipital to central sites (O2 to $\mathrm{Cz}: 15-35 \mathrm{~Hz})$ and from central to the left inferior-temporal sites (Cz to P7: 4-45Hz), while stronger connectivity for symbols was present from central to the left frontal sites ( $\mathrm{Cz}$ to $\mathrm{F} 5$ : $37-47 \mathrm{~Hz}$ ). In the severely dysfluent dyslexic group, words evoked stronger connectivity from O2 to P7 $(40-49 \mathrm{~Hz}, 59-70 \mathrm{~Hz})$. Also in this group symbols evoked more widespread connectivity increases in the posterior to anterior direction from P7 to $\mathrm{Cz}(20$ $39 \mathrm{~Hz}$ ) and $\mathrm{C} 3(12-45 \mathrm{~Hz})$, stronger anterior to posterior connectivity from $\mathrm{F} 6$ to P8 $(61-70 \mathrm{~Hz}), \mathrm{Cz}(50-63 \mathrm{~Hz}), \mathrm{C} 3(56-67 \mathrm{~Hz})$ and cross-hemispherically to F5 $(26-50 \mathrm{~Hz}, 52-70 \mathrm{~Hz})$.

\subsection{Group differences in EEG connectivity patterns for visual words}

Group differences in connectivity during the recognition of the words indicated specific patterns of posterior to anterior and anterior to posterior connectivity dependent on the level of reading fluency of the participants.

We first focus on connectivity results in the posterior to anterior direction. In both dyslexic groups EEG responses to words showed weaker connectivity than those of typical readers from the left occipital site to bilateral inferiortemporal sites. Thus, in MDDs as compared to TRs (Fig. 4-4a; Fig. 4-5, upper row) we observed a weaker connectivity from O1 to P7 $(52-68 \mathrm{~Hz})$ and P8 (58$70 \mathrm{~Hz}$ ). Similarly, in SDDs as compared to TRs (Fig. 4-4b; Fig. 4-5, middle row) we observed weaker connectivity from O1 to P7 $(61-70 \mathrm{~Hz})$ and P8 $(59-70 \mathrm{~Hz})$. Opposite to these differences between dyslexic and typical readers, moderately dysfluent dyslexics showed stronger connectivity from occipital to central sites than both other groups. Thus, MDDs showed stronger connectivity from O1 to $\mathrm{Cz}$ than both TRs $(12-49 \mathrm{~Hz})$ and SDDs $(5-46 \mathrm{~Hz})$, and stronger connectivity from O2 to $\mathrm{Cz}(13-56 \mathrm{~Hz})$ and $\mathrm{O} 2$ to $\mathrm{C} 3(24-53 \mathrm{~Hz})$ than TRs (Fig. 4-4c; Fig. 45 , bottom row). Finally, the SDD group showed stronger connectivity than TRs from O2 to $\mathrm{Cz}(27-41 \mathrm{~Hz}, 43-61 \mathrm{~Hz}), \mathrm{C} 3(23-60 \mathrm{~Hz})$ and $\mathrm{C} 4(28-68 \mathrm{~Hz})$. 
Both groups of dyslexic children showed stronger anterior to posterior connectivity than the typically reading children, in particular from left frontal to bilateral occipital and right inferior-temporal sites. Thus, MDDs had stronger connectivity than TRs from F5 to O1 $(19-70 \mathrm{~Hz}), \mathrm{O} 2(23-70 \mathrm{~Hz}), \mathrm{P} 8(1-70 \mathrm{~Hz})$, and $\mathrm{Cz}(19-70 \mathrm{~Hz})$, while SDDs had stronger connectivity than TRs from F5 to O1 $(8-70 \mathrm{~Hz}), \mathrm{O} 2(62-70 \mathrm{~Hz})$ and P8 $(26-70 \mathrm{~Hz})$. In addition, SDDs showed stronger connectivity from right frontal to bilateral occipital sites than MDDs, i.e. from F6 to $\mathrm{O} 1(1-30 \mathrm{~Hz}), \mathrm{O} 2(4-21 \mathrm{~Hz})$ and $\mathrm{C} 4(1-62 \mathrm{~Hz})$.

Finally in anterior to posterior connectivity patterns starting from the central sites we observed stronger connectivity towards occipital and inferior-temporal sites in both dyslexic groups as compared to typical readers. While in the SDD group this was constrained to the right side of the head, in the MDD group this increased connectivity was observed bilaterally. Thus, MDDs showed stronger connectivity than TRs from Cz to O1 $(22-60 \mathrm{~Hz}), \mathrm{O} 2(20-63 \mathrm{~Hz}), \mathrm{P} 7(26-57 \mathrm{~Hz})$ and P8 (26-61Hz), and from C3 to O2 $(27-50 \mathrm{~Hz})$ and $\mathrm{Cz}(30-56 \mathrm{~Hz})$. SDDs instead showed stronger connectivity than TRs from $\mathrm{Cz}$ to $\mathrm{O} 2(31-66 \mathrm{~Hz})$ and P8 $(24-70 \mathrm{~Hz})$, and from C3 to O2 $(18-55 \mathrm{~Hz})$ and P8 $(4-56 \mathrm{~Hz})$. In the left hemisphere, the connectivity from central sites was stronger in MDDs than SDDs (Cz to P7: 5-47Hz). Finally, SDDs had stronger lateral connectivity than TRs from central sources, i.e. from $\mathrm{Cz}$ to $\mathrm{C} 3$ and $\mathrm{C} 4(\mathrm{Cz}-\mathrm{C} 3: 21-33 \mathrm{~Hz}, 35-$ $50 \mathrm{~Hz}$; $\mathrm{Cz}-\mathrm{C} 4:$ 19-70Hz), and from C3 to $\mathrm{Cz}$ and $\mathrm{C} 4$ (C3-Cz: 7-50Hz; C3-C4: $12-41 \mathrm{~Hz})$.

In summary, during the processing of visual words, both dyslexic groups showed weaker connectivity from occipital to temporal sites than the typical readers. Furthermore, both dyslexic groups showed stronger connectivity towards and from central sites, and this connectivity scaled with the level of dysfluency. Moderately dysfluent dyslexics had stronger connectivity towards $\mathrm{Cz}$ and bilateral connectivity from central towards inferior-temporal sites, while severely dysfluent dyslexics showed stronger connectivity only towards the right inferior-temporal site. Finally, both groups of dyslexics exhibited stronger connectivity from the left frontal to the right inferior-temporal site. 


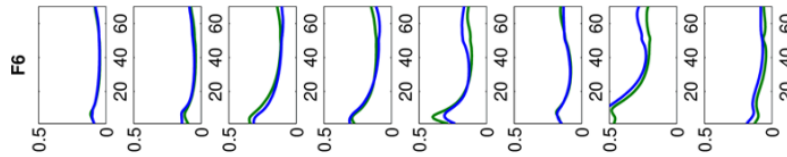

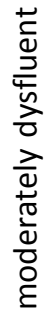

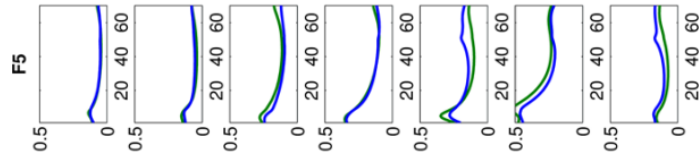

$\int_{0}^{0}$
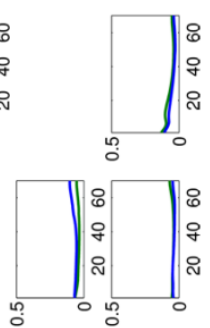

这
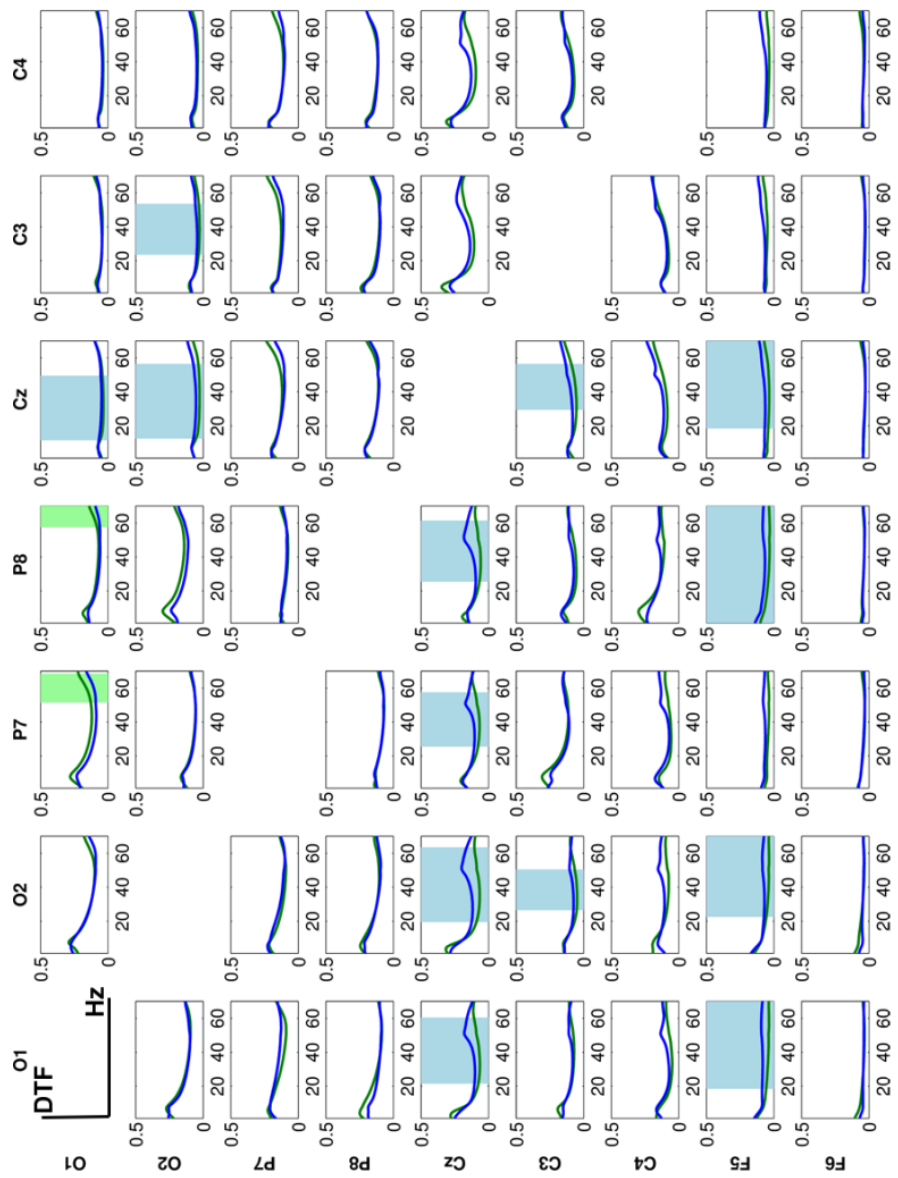

adw / لا 
Reading network connectivity and dysfluency

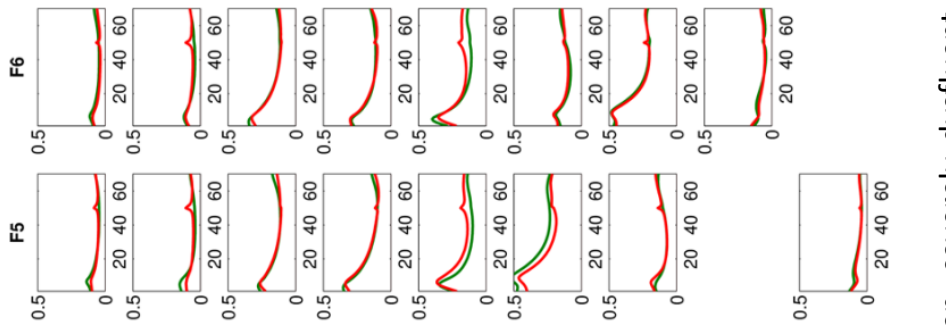

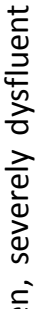
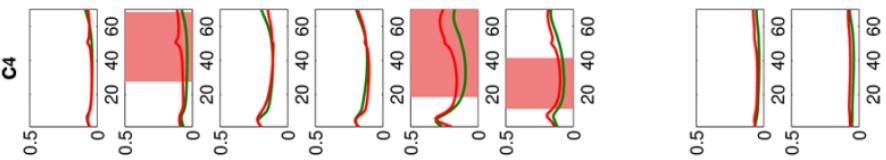

ฮั่
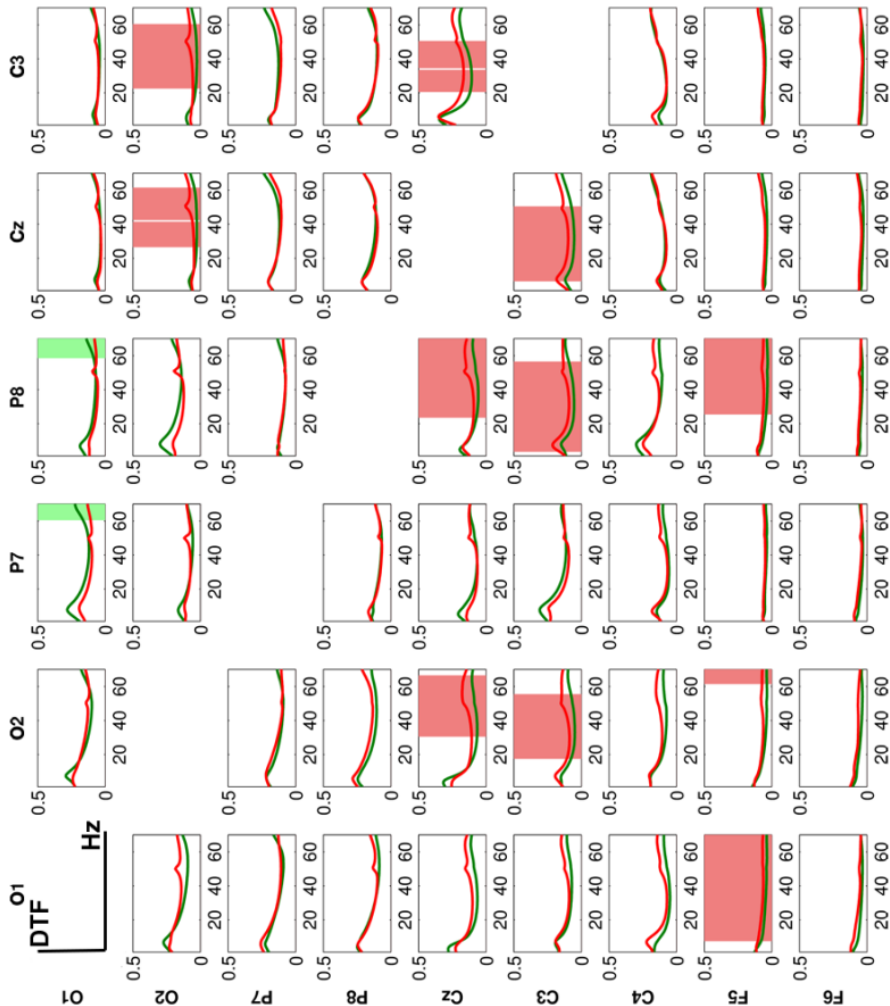

ads / لا

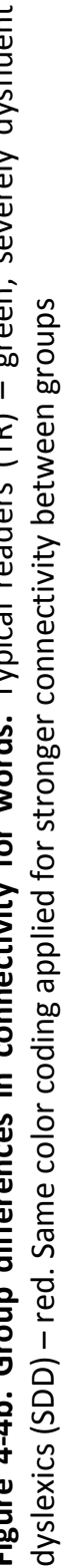



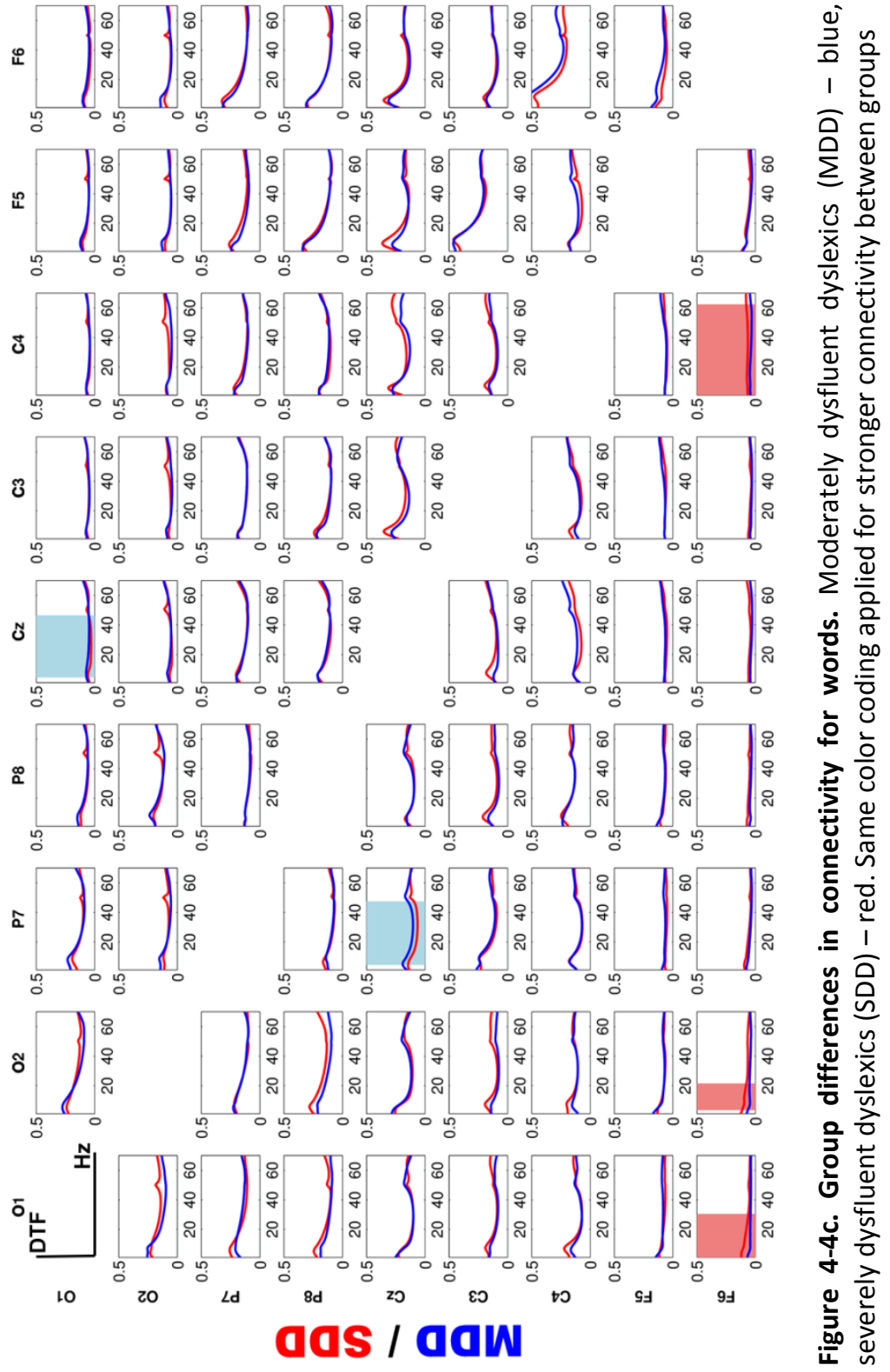


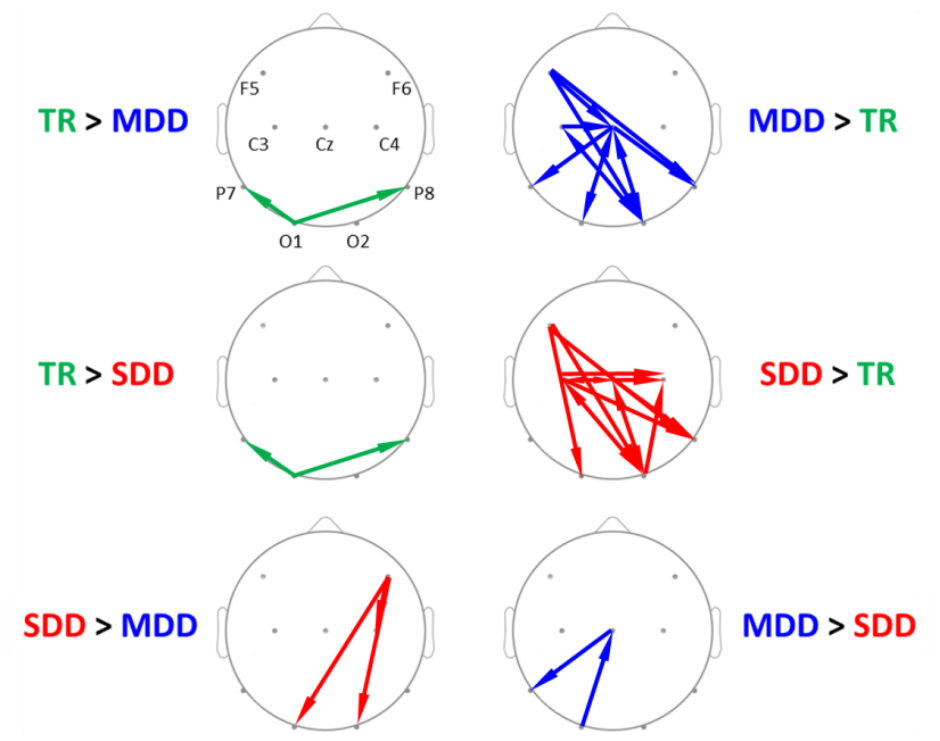

Figure 4-5. Topographic visualization of group differences in connectivity for words. Stronger connectivity in: typical readers (TR) - green, moderately dysfluent dyslexics (MDD) - blue, severely dysfluent dyslexics (SDD) - red. Arrows - direction of the connectivity.

\subsection{Group differences in EEG connectivity patterns for symbol strings}

Group comparisons of connectivity during processing of meaningless false font symbol-strings mainly yielded differences between severely dysfluent dyslexics and the other two groups, while differences between typical and moderately dysfluent dyslexics were diminished.

Posterior to anterior connectivity patterns in EEG activity elicited by symbol strings showed weaker connectivity from right occipital towards right inferiortemporal in moderately dysfluent dyslexics relative to typical readers, i.e. from O2 to P8 (13-49Hz; Fig. 4-6a; Fig. 4-7, upper row). MDD instead showed stronger occipital and central to right frontal connectivity than TRs, i.e. from O1 to F6 (40-58Hz) and from C4 to F6 (50-52Hz). Severely dysfluent dyslexic readers showed a more widespread pattern of weaker connectivity than typical 
readers (Fig. 4-6b; Fig. 4-7, middle row) from bilateral occipital to bilateral inferior-temporal sites: from $\mathrm{O} 2$ to $\mathrm{P} 7$ and P8 (O2-P7:1-7Hz, 61-70Hz; O2-P8: 3-16Hz), and from O1 to P7, P8 and C3 (O1-P7: 3-5Hz, 53-70Hz; O1-P8: 6$11 \mathrm{~Hz}, 33-44 \mathrm{~Hz}, 58-70 \mathrm{~Hz}$; O1-C3: 1-23Hz, 60-70Hz). Furthermore, SDDs showed weaker connectivity than MDDs (Fig. 4-6c; Fig. 4-7, bottom row) from left occipital to more central and frontal sites, i.e. from O1 to $\mathrm{Cz}(4-46 \mathrm{~Hz}, 61-$ $70 \mathrm{~Hz}), \mathrm{C} 3(5-8 \mathrm{~Hz}, 65-70 \mathrm{~Hz}), \mathrm{F} 5(3-11 \mathrm{~Hz})$ and $\mathrm{F} 6(8-18 \mathrm{~Hz}, 26-66 \mathrm{~Hz})$. Conversely, SDDs had stronger connectivity than MDDs from O2 to O1 (18$44 \mathrm{~Hz})$ and $\mathrm{C} 4(14-46 \mathrm{~Hz}, 49-59 \mathrm{~Hz})$.

Anterior to posterior connectivity was observed to be stronger for SDDs than both other groups, especially towards the right posterior regions. Thus, SDDs had stronger connectivity than TRs from F5, F6, and C4 to O1 (F5-O1: 6570Hz; F6-O1: $57-68 \mathrm{~Hz}, \mathrm{C} 4-\mathrm{O} 1: 6-11 \mathrm{~Hz})$, from F6 to $\mathrm{C} 4(14-30 \mathrm{~Hz})$, and from $\mathrm{C} 3$ and $\mathrm{Cz}$ to $\mathrm{O} 2$ and P8 (C3-O2: 15-70Hz; C3-P8: 31-58Hz; Cz-O2: 38-63Hz; Cz-P8: 36-58Hz). Furthermore, SDDs had stronger connectivity than MDDs from F6 to C4 and P8 (F6-C4: 8-70Hz; F6-P8: 61-70Hz), and from C4 and C3 to $\mathrm{O} 2$ (C4-O2: 9-18Hz; C3-O2: 10-70Hz). Finally, SDDs had stronger lateral connectivity at frontal sites than the other two groups, i.e. from F6 to F5 as compared to TRs (39-70Hz) and MDDs (29-70Hz).

In summary, while the differences between moderately dysfluent dyslexics and typical readers were strongly reduced as compared to the word condition, both dyslexic groups still exhibited weaker occipito-temporal connectivity than typical readers during the processing of false font symbols. Interestingly, while this difference was restricted to the right side of the head for moderately dysfluent dyslexics versus typical readers, it occurred bilaterally for severely dysfluent dyslexics versus typical readers. Diminished differences between moderately dysfluent dyslexics and typical readers may suggest a qualitatively similar processing of false font symbol strings. The stronger anterior to posterior connectivity in severely dysfluent dyslexics may instead represent a different processing of false font symbols, involving similar network interactions as those recruited during the processing of visual words. 
Reading network connectivity and dysfluency

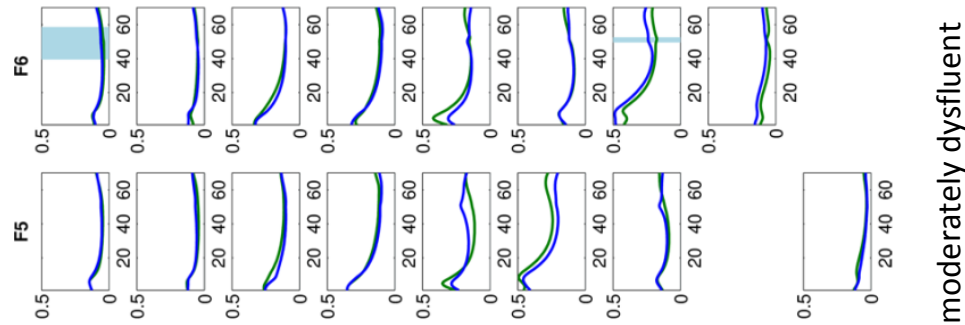

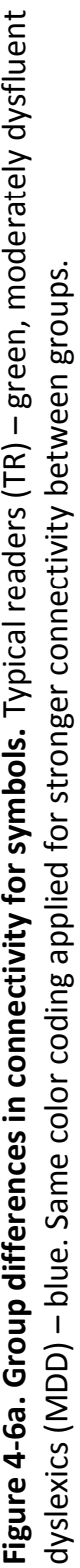


눈
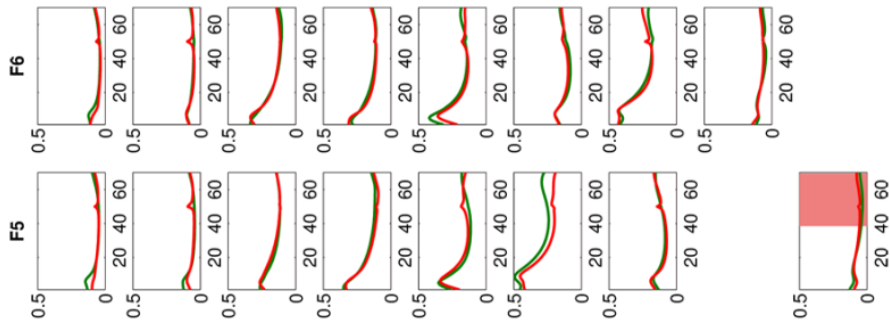

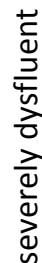
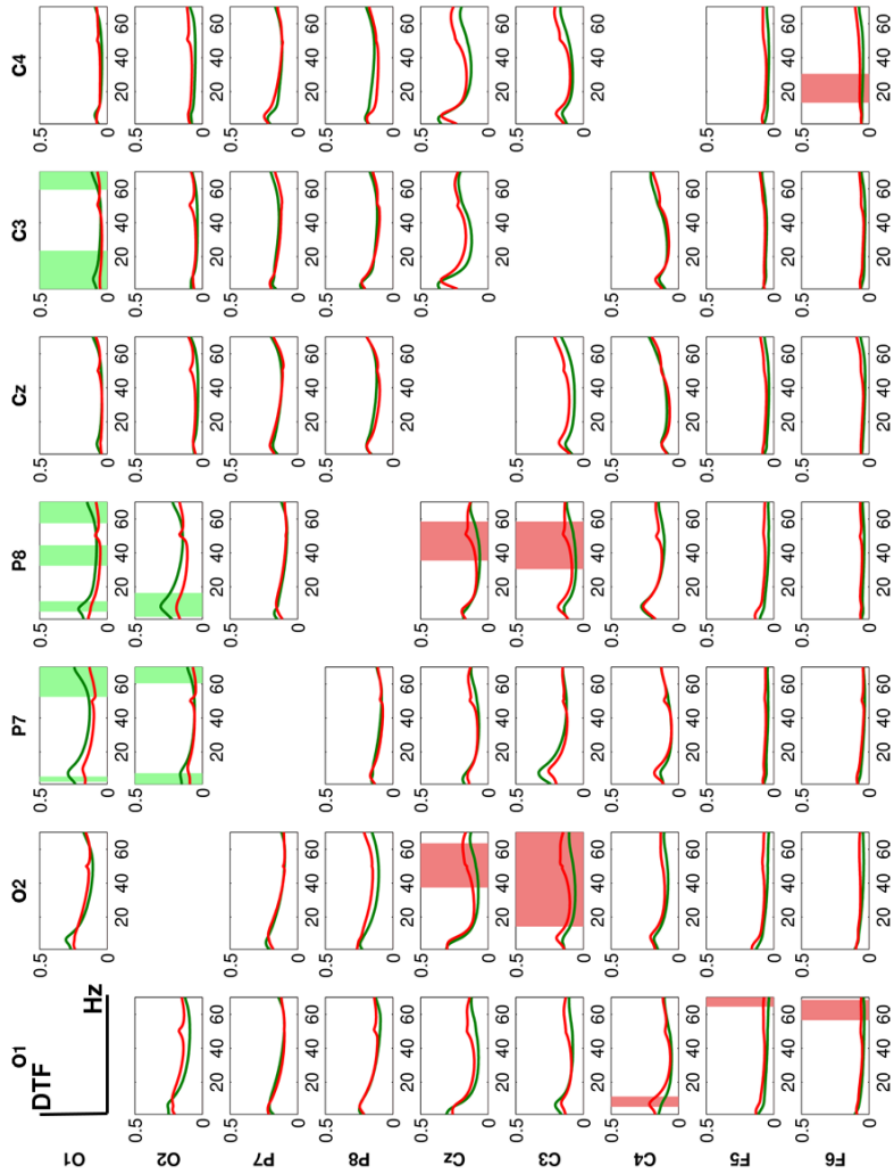

ads / لا لـ

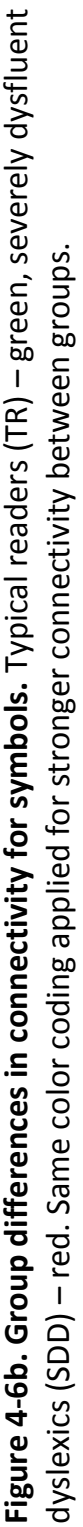


Reading network connectivity and dysfluency

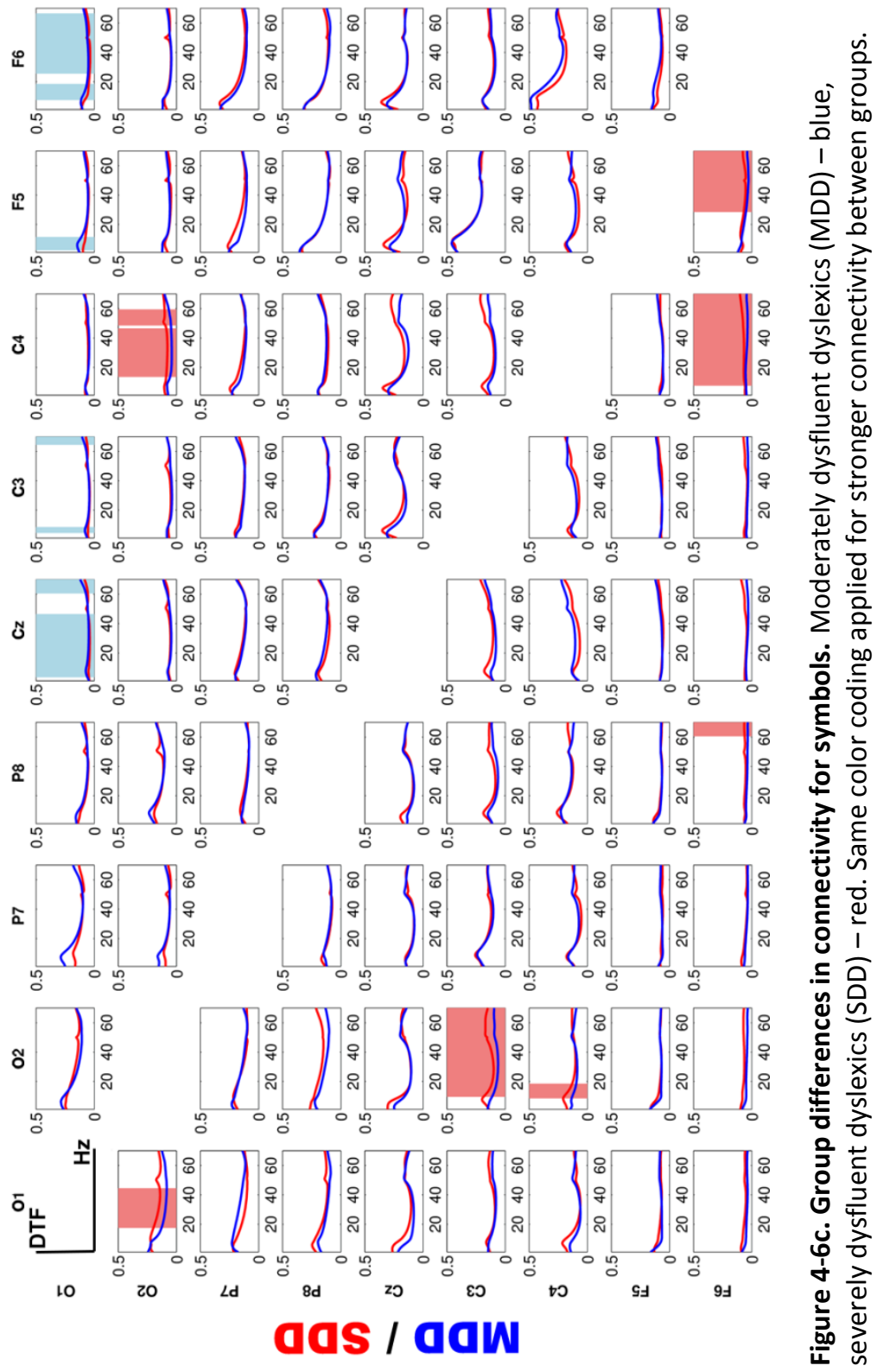




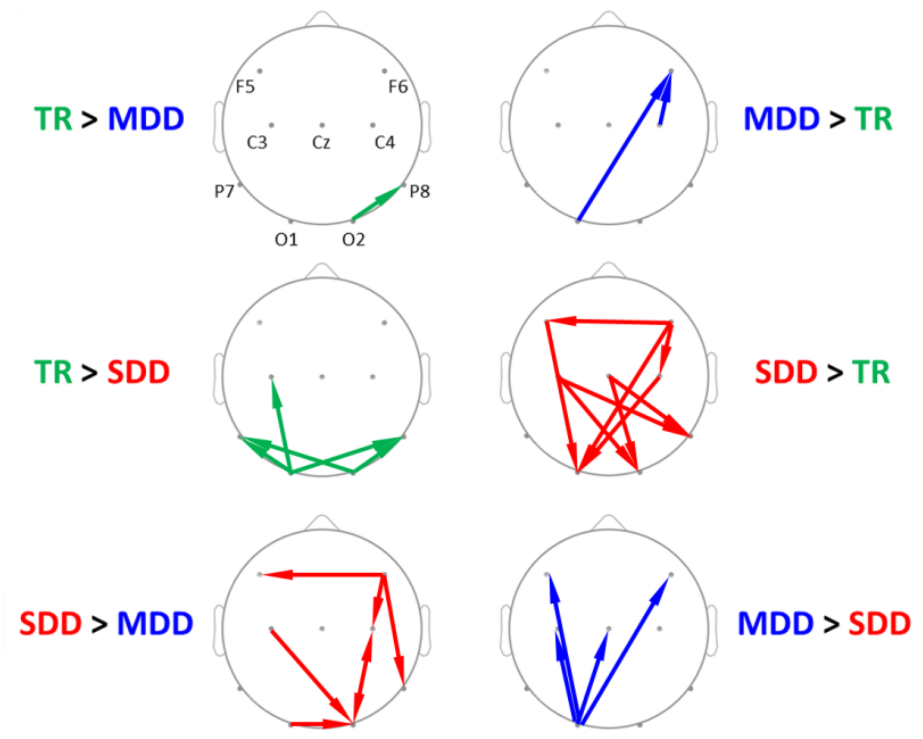

Figure 4-7. Topographic visualization of group differences in connectivity for symbols. Stronger connectivity in: typical readers (TR) - green, moderately dysfluent dyslexics (MDD) - blue, severely dysfluent dyslexics (SDD) - red. Arrows direction of the connectivity.

\section{Discussion}

In the present study, we examined EEG effective connectivity during visual word and symbol processing in 9-year old typically reading and dyslexic children differing in the severity of their reading dysfluency. To this end we calculated connectivity between EEG channels during a visual word recognition paradigm. Firstly, our results indicate fluency dependent differences in posterior-anterior connectivity with weaker connectivity from occipital to occipito-temporal sites in both dyslexic groups relative to typical readers, and stronger connectivity from occipital to central (and frontal) sites in moderately dysfluent dyslexic than severely dysfluent dyslexic children. Secondly, anteriorposterior connectivity scaled with reading fluency. Moderately dysfluent dyslexics showed stronger connectivity to bilateral occipito-temporal sites, and severely dysfluent dyslexics to right occipito-temporal sites. Finally, the differences between moderately dysfluent dyslexics and typical readers were 
strongly reduced in the false font condition, while these groups still differed from the severely dysfluent dyslexic group.

\subsection{Bottom-up progression of information (from occipital to temporal regions)}

Both groups of dyslexic children exhibited weaker occipital to inferior-temporal connectivity, suggesting a reduced information transfer between early and more specialized visual areas. Thus, the processing of visually presented words has been found to start in primary visual areas and proceed through higher visual cortex ventrally to the left occipito-temporal sulcus, where the putative visual word form area is located (Dehaene, Cohen, Sigman, \& Vinckier, 2005; McCandliss et al., 2003). Connectivity MEG/EEG studies investigating reading found a pattern of posterior to frontal activity propagation combined with topdown guided processing throughout the network (Kujala et al., 2007), and more specifically, a feedforward/feedback interplay between occipito-temporal, superior-temporal and inferior-frontal regions (Bedo, Ribary, \& Ward, 2014). Furthermore, similar to our results, a recent fMRI connectivity study found weaker functional connectivity along the visual pathway in 9 year old dyslexics relatively to their typically reading peers (Finn et al., 2014). Similarly, a study investigating phonological processing with a non-word rhyming task found weaker connectivity for dyslexic readers between the primary visual cortex and the left angular gyrus, whereas the same connectivity was stronger for dyslexic readers in the right hemisphere (Pugh et al., 2000).

\subsection{Stronger connectivity towards and from central sites in dyslexic groups}

Both groups of dyslexics showed stronger posterior to anterior connectivity than typical readers towards central sites, with stronger connectivity towards $\mathrm{Cz}$ in moderately dysfluent dyslexic as compared to severely dyslexic readers. In addition, both groups of dyslexics showed stronger connectivity towards and from central sites. In a previous study using an audiovisual oddball paradigm, both dyslexic groups were shown to exhibit reduced neural integration of

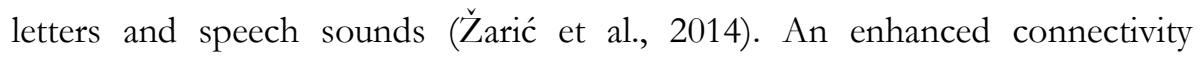


towards and from central sites may thus reflect the involvement of auditory, sensorimotor regions and/or verbal working memory in an attempt to connect orthography to phonology (Blomert, 2011; Pugh et al., 2001; Simos et al., 2002; van Atteveldt \& Ansari, 2014). Accordingly, an increase of coherence with and between these central sites is also seen in dyslexics after neurofeedback training, together with improvements in reading and phonological awareness (Nazari, Mosanezhad, Hashemi, \& Jahan, 2012), while the lack of reading improvement was coupled with the lack of changes in a fronto-central coherence (Breteler, Arns, Peters, Giepmans, \& Verhoeven, 2010). Similarly, in typical development, greater activation of regions at the temporo-parietal junction, including the supramarginal gyrus, in 9 year old children than in adult readers is taken as a sign of greater reliance of children on phonological processing during reading (Booth et al., 2001; Church, Coalson, Lugar, Petersen, \& Schlaggar, 2008). Such developmental changes may relate to a gradual shift from letter by letter to whole word reading with advancing reading skills (Ehri, 2005; Share, 1995). Hence, while 9 years old typical readers may already shift to the whole word reading for highly familiar words (age of acquisition - 6 years), both groups of dyslexics may still be reading in a letter by letter fashion due to a lack of automaticity in letter-speech sound integration (Blomert, 2011; Ehri, 2005; Share, 1995; Wimmer \& Schurz, 2010) resulting in a reading fluency that falls in the lowest 10 percentiles (Žarić et al., 2014). Although speculative at this point, this interpretation would also be consistent with weaker occipital to inferiortemporal connectivity indicating decreased recruitment of the putative visual word form area (Jobard et al., 2003; McCandliss et al., 2003). Finally, moderately dysfluent children may have more efficient compensatory mechanisms related to a stronger connectivity in this group from central sites towards bilateral inferior temporal sites. The engagement of left side (hemisphere) sites, which are typically associated to a higher specialization for reading (e.g. Yamada et al., 2011) may relate to fluency differences between moderately and severely dysfluent dyslexics, whose compensatory mechanisms are restricted to the right inferior temporal site. 


\subsection{Top-down progression of information (from frontal to occipital and right inferior-temporal regions)}

A next important finding of this study is the different pattern of anterior to posterior connectivity for word reading in the two groups of dyslexic children. Thus, both groups showed stronger anterior to posterior connectivity from the left frontal to the right inferior-temporal site, and severely dysfluent dyslexics additionally engaged the right frontal site relative to the moderately dysfluent dyslexics. The stronger connectivity from inferior frontal to posterior sites in dyslexic readers is in line with previous research showing increased fMRI connectivity during resting state and reading related tasks between the left IFG and multiple brain areas, e.g. medial prefrontal cortex, anterior cingulate, and left caudate (Finn et al., 2014), bilateral inferior parietal lobule/angular gyrus and right rolandic operculum (Schurz et al., 2014), right and left middle frontal gyrus, right and left supplementary motor area, the left precentral gyrus, and the right superior frontal gyrus (Richards \& Berninger, 2008). The stronger connectivity from the left IFG in dyslexics may suggest more effortful strategies based on phonology, such as sounding out, during reading in order to compensate the disruption in posterior-anterior connectivity that halts conversion to more rapid visual word recognition (Finn et al., 2014).

Interestingly, it appears that the dependency on right hemisphere sites increases as fluency decreases. While both dyslexic groups showed stronger connectivity for words than typical readers from left frontal sites to right inferior-temporal sites, severely dysfluent dyslexics additionally seemed to more strongly involve right frontal sites in the false font symbols. Furthermore in the severely dysfluent dyslexics we observed a stronger propagation from central towards the right inferior temporal site only, while moderately dysfluent dyslexics showed stronger bilateral central to inferior-temporal site connectivity. These findings confirm and extend previous fMRI studies reporting stronger connectivity in 9-year old dyslexic readers within the right hemisphere (Finn et al., 2014), and hyperactivation of right frontal (Grünling et al., 2004; Ingvar et al., 2002) and right occipito-temporal areas (Ingvar et al., 2002) in dyslexics. This stronger frontal to right inferior-temporal connectivity could represent a 
crucial compensatory mechanism of right hemisphere homologues of word processing in virtue of a diminished left-sided posterior to anterior connectivity.

In addition to word related differences, the presence of a stronger anterior to posterior connectivity was separating severely dysfluent dyslexics from the other two groups in the false font condition. This could suggest that the severely dysfluent dyslexics processed the quasi-orthographic material similarly as words. The strongest connectivity from the right frontal site in the severely dysfluent dyslexic group is reminiscent of a significant negative correlation between fMRI activation in the right frontal areas and reading skills (Ingvar et al., 2002). Interestingly, the severely dysfluent dyslexic group was previously shown to exhibit a more severe impairment in letter-speech sound integration than the moderately dyslfuent group, especially in an early time window associated with an impaired forming of a clear letter-speech sound representation (Žarić et al., 2014). The observation of substantial group differences with severely dysfluent dyslexics for both orthographic (words) and quasi-orthographic material (false font) may similarly indicate reduced bottomup differentiation of letter strings (words) and false font symbols due to a lack of firm connections of letters to speech sounds. We recently reported that a letter-speech sound training in dyslexic children could affect reading fluency and a measure of neural letter-speech sound integration (Žarić et al., 2015). It would thus be interesting to further investigate whether letter-speech sound training induces brain connectivity changes in the same direction as those found in the present study.

Finally, visual words as compared to symbol strings only yielded small differences in the overall connectivity patterns over frequencies, with more widespread connectivity for symbols in typical and severely dysfluent dyslexics. This lack of large differences could be a consequence of the relatively long interval used in the connectivity analysis, i.e 0 - 900 ms. A recent MEG study showed that differences between words and false fonts depended on narrower time windows, e.g. stronger connectivity from left occipital cortex to left 
ventral occipital cortex and left inferior frontal gyrus (IFG) for words than for false font in a $300 \mathrm{~ms}$ window (Woodhead et al., 2014). Our longer window could attenuate these differences due to their shorter duration. Alternatively, differences in connectivity between the words and the false font condition could be attenuated by longer lasting cognitive states induced by the repetition of stimuli from the same category within blocks (Al-Aidroos, Said, \& TurkBrowne, 2012). Because ERP results on a subset of children included in this study revealed temporally-selective ERP differences for both type (words and symbols) and length (short and long) factors (Fraga González et al., 2014), it would be interesting to further investigate the present EEG data using time variant connectivity measures, such as the short-time directed transfer function (Blinowska, 2011).

\subsection{Conclusions}

According to our knowledge, this is the first study that investigates effective functional connectivity between dyslexic children of the variable fluency and compares them to typically reading children. Our results point to different connectivity patterns in the reading network between typical and dyslexic children and within dyslexic children as a function of the severity of their reading dysfluency.

\section{References}

Al-Aidroos, N., Said, C. P., \& Turk-Browne, N. B. (2012). Top-down attention switches coupling between low-level and high-level areas of human visual cortex. Proceedings of the National Academy of Sciences, 109(36), 14675-14680. doi:10.1073/pnas.1202095109

Androulidakis, A. G., Mazzone, P., Litvak, V., Penny, W., Dileone, M., Gaynor, L. M. F. D., ... Brown, P. (2008). Oscillatory activity in the pedunculopontine area of patients with Parkinson's disease. Experimental Neurology, 211(1), 59-66.

doi:10.1016/j.expneurol.2008.01.002

Arns, M., Peters, S., Breteler, R., \& Verhoeven, L. (2007). Different brain activation patterns in dyslexic children: evidence from EEG power and coherence patterns for the doubledeficit theory of dyslexia. Journal of Integrative Neuroscience, 6(1), 175-90. Retrieved from http://www.ncbi.nlm.nih.gov/pubmed/17472228 
Baccalá, L. a, \& Sameshima, K. (2001). Partial directed coherence: a new concept in neural structure determination. Biological Cybernetics, 84(6), 463-74. Retrieved from http://www.ncbi.nlm.nih.gov/pubmed/11417058

Bedo, N., Ribary, U., \& Ward, L. M. (2014). Fast dynamics of cortical functional and effective connectivity during word reading. PLoS ONE, 9(2). doi:10.1371/journal.pone.0088940

Benjamini, Y., \& Hochberg, Y. (1995). Controlling the false discovery rate: a practical and powerful approach to multiple testing. Journal of the Royal Statistical Society. Series B (Methodological), 57(1), 289-300. Retrieved from http://www.jstor.org/stable/10.2307/2346101

Blinowska, K. J. (2011). Review of the methods of determination of directed connectivity from multichannel data. Medical and Biological Engineering and Computing, 49(5), 521-529. doi:10.1007/s11517-011-0739-x

Blinowska, K. J., Kamiński, M., Brzezicka, A., \& Kamiński, J. (2013). Application of directed transfer function and network formalism for the assessment of functional connectivity in working memory task. Philosophical Transactions. Series A, Mathematical, Physical, and Engineering Sciences, 371(1997), 20110614. doi:10.1098/rsta.2011.0614

Blinowska, K. J., Kuś, R., \& Kamiński, M. (2004). Granger causality and information flow in multivariate processes. Physical Review E, 70(5), 050902. doi:10.1103/PhysRevE.70.050902

Blinowska, K. J., Kuś, R., Kaminski, M., \& Janiszewska, J. (2010). Transmission of brain activity during cognitive task. Brain Topography, 23(2), 205-213. doi:10.1007/s10548-010-0137-y

Blomert, L. (2005). Dyslexie in Nederland. Amsterdam: Uitgeverij Nienwerijds. Amsterdam: Uitgeverij Nieuwezijds. Retrieved from http://www.boomtestuitgevers.nl/upload/Dyslexie_in_Nederland_Leo_Blomert.pdf

Blomert, L. (2011). The neural signature of orthographic-phonological binding in successful and failing reading development. NeuroImage, 57(3), 695-703. doi:10.1016/j.neuroimage.2010.11.003

Blomert, L., \& Vaessen, A. A. (2009). 3DM Differential diagnostics for dyslexia: Cognitive analysis of reading and spelling. Amsterdam: Boom Test Publishers.

Booth, J. R., Burman, D. D., Van Santen, F. W., Harasaki, Y., Gitelman, D. R., Parrish, T. B., \& Marsel Mesulam, M. M. (2001). The development of specialized brain systems in reading and oral-language. Child Neuropsychology: A Journal on Normal and Abnormal Development in Childhood and Adolescence, 7(3), 119-41. doi:10.1076/chin.7.3.119.8740

Breteler, M. H. M., Arns, M., Peters, S., Giepmans, I., \& Verhoeven, L. (2010). Improvements in spelling after QEEG-based neurofeedback in dyslexia: a randomized controlled treatment study. Applied Psychophysiology and Biofeedback, 35(1), 5-11. doi:10.1007/s10484-009-9105-2

Brus, B. T., \& Voeten, M. J. M. (1973). Eén-minunt test, vorm A en B; Verantwoording en Handleiding. Nimegen: Berkenhout Testmateriaal. 


\section{Reading network connectivity and dysfluency}

Brzezicka, A., Kamiński, M., Kamiński, J., \& Blinowska, K. J. (2011). Information transfer during a transitive reasoning task. Brain Topography, 24(1), 1-8. doi:10.1007/s10548-010-0158-6

Church, J. a., Coalson, R. S., Lugar, H. M., Petersen, S. E., \& Schlaggar, B. L. (2008). A developmental fMRI study of reading and repetition reveals changes in phonological and visual mechanisms over age. Cerebral Cortex, 18(9), 2054-2065. doi: $10.1093 /$ cercor/bhm 228

Cui, J., Xu, L., Bressler, S. L., Ding, M., \& Liang, H. (2008). BSMART: A Matlab/C toolbox for analysis of multichannel neural time series. Neural Networks, 21(8), 1094-1104. doi:10.1016/j.neunet.2008.05.007

Dambacher, M., Dimigen, O., Braun, M., Wille, K., Jacobs, A. M., \& Kliegl, R. (2012). Stimulus onset asynchrony and the timeline of word recognition: event-related potentials during sentence reading. Neuropsychologia, 50(8), 1852-70. doi:10.1016/j.neuropsychologia.2012.04.011

de Vos, T. (2007). Schoolvaardigheidstoets Technisch Lezen. Amsterdam: Boom test uitgevers.

Dehaene, S., Cohen, L., Sigman, M., \& Vinckier, F. (2005). The neural code for written words: A proposal. Trends in Cognitive Sciences, 9(7), 335-341. doi:10.1016/j.tics.2005.05.004

Delorme, A., \& Makeig, S. (2004). EEGLAB: an open source toolbox for analysis of single-trial EEG dynamics including independent component analysis. Journal of Neuroscience Methods, 134, 9-21. Retrieved from http://www.sciencedirect.com/science/article/pii/S0165027003003479

Dimitriadis, S. I., Laskaris, N. a., Simos, P. G., Micheloyannis, S., Fletcher, J. M., Rezaie, R., \& Papanicolaou, a. C. (2013). Altered temporal correlations in resting-state connectivity fluctuations in children with reading difficulties detected via MEG. NeuroImage, 83, 307317. doi:10.1016/j.neuroimage.2013.06.036

Ehri, L. C. (2005). Learning to Read Words: Theory, Findings, and Issues. Scientific Studies of Reading, 9(2), 167-188. doi:10.1207/s1532799xssr0902_4

Engel, a K., Fries, P., \& Singer, W. (2001). Dynamic predictions: oscillations and synchrony in top-down processing. Nature Reviews. Neuroscience, 2(October), 704-716. doi: $10.1038 / 35094565$

Ewen, J. B., Lakshmanan, B. M., Hallett, M., Mostofsky, S. H., Crone, N. E., \& Korzeniewska, A. (2015). Dynamics of functional and effective connectivity within human cortical motor control networks. Clinical Neurophysiology: Official Journal of the International Federation of Clinical Neurophysiology, 126(5), 987-96. doi:10.1016/j.clinph.2014.09.006

Finn, E. S., Shen, X., Holahan, J. M., Scheinost, D., Lacadie, C., Papademetris, X., ... Constable, R. T. (2014). Disruption of functional networks in dyslexia: A whole-brain, data-driven analysis of connectivity. Biological Psychiatry, 76(5), 397-404. doi:10.1016/j.biopsych.2013.08.031

Fraga González, G., Van der Molen, M. J. W., Žarić, G., Bonte, M., Tijms, J., Blomert, L., .. van 
der Molen, M. W. (n.d.). Graph Analysis of EEG Resting State Functional Networks in Dyslexic and Typically Reading Children.

Fraga González, G., Žarić, G., Tijms, J., Bonte, M., Blomert, L., \& van der Molen, M. W. (2014). Brain-potential analysis of visual word recognition in dyslexics and typically reading children. Frontiers in Human Neuroscience, 8(June), 1-14. doi:10.3389/fnhum.2014.00474

Franaszczuk, P. J., Bergey, G. K., \& Kamiński, M. J. (1994). Analysis of mesial temporal seizure onset and propagation using the directed transfer function method. Electroencephalography and Clinical Neurophysiology, 91(6), 413-427. doi:10.1016/0013-4694(94)90163-5

Geschwind, N. (1965). Disconnexion syndromes in animals and man. I. Brain, 88(2), 237-294. doi:10.1093/brain/88.2.237

Ghyselinck, M., De Moor, W., \& Brysbaert, M. (2000). Age-of-acquisition ratings for 2816 Dutch four- and five-letter nouns. Psychologica Belgica, 40(2), 77-98. Retrieved from https://archive.ugent.be/record/132371

Gibbons, J. D., \& Chakraborti, S. (2003). Nonparametric statistical inference. (J. D. Gibbons \& S. Chakraborti, Eds.) (4th ed.). New York, NY: Marcel Dekker, Inc.

Ginter, J., Blinowska, K. J., Kamiński, M., \& Durka, P. J. (2001). Phase and amplitude analysis in time-frequency space--application to voluntary finger movement. Journal of Neuroscience Methods, 110(1-2), 113-124. doi:10.1016/S0165-0270(01)00424-1

Granger, C. W. J. (1969). Investigating Causal Relations by Econometric Models and Crossspectral Methods. Econometrica, 37(3), 424. doi:10.2307/1912791

Grünling, C., Ligges, M., Huonker, R., Klingert, M., Mentzel, H.-J., Rzanny, R., ... Blanz, B. (2004). Dyslexia: the possible benefit of multimodal integration of fMRI- and EEG-data. Journal of Neural Transmission (Vienna, Austria : 1996), 111, 951-969. doi:10.1007/s00702004-0117-z

Hannagan, T., Amedi, A., Cohen, L., Dehaene-lambertz, G., \& Dehaene, S. (2015). Origins of the specialization for letters and numbers in ventral occipitotemporal cortex. Trends in Cognitive Sciences, 1-9. doi:10.1016/j.tics.2015.05.006

Hoeft, F., Meyler, A., Hernandez, A., Juel, C., Taylor-Hill, H., Martindale, J. L., ... Gabrieli, J. D. E. (2007). Functional and morphometric brain dissociation between dyslexia and reading ability. Proceedings of the National Academy of Sciences of the United States of America, 104(10), 4234-4239. doi:10.1073/pnas.0609399104

Ingvar, M., Af Trampe, P., Greitz, T., Eriksson, L., Stone-Elander, S., \& Von Euler, C. (2002). Residual differences in language processing in compensated dyslexics revealed in simple word reading tasks. Brain and Language, 83(2), 249-267. doi:10.1016/S0093934X(02)00055-X

Jobard, G., Crivello, F., \& Tzourio-Mazoyer, N. (2003). Evaluation of the dual route theory of reading: A metanalysis of 35 neuroimaging studies. NeuroImage, 20, 693-712. doi:10.1016/S1053-8119(03)00343-4 


\section{Reading network connectivity and dysfluency}

Jung, T. P., Makeig, S., Humphries, C., Lee, T. W., McKeown, M. J., Iragui, V., \& Sejnowski, T. J. (2000). Removing electroencephalographic artifacts by blind source separation. Psychophysiology, 37(2), 163-78. Retrieved from http://www.ncbi.nlm.nih.gov/pubmed/10731767

Kaminski, M., \& Blinowska, K. J. (2014). Directed Transfer Function is not influenced by volume conduction-inexpedient pre-processing should be avoided. Frontiers in Computational Neuroscience, 8(June), 61. doi:10.3389/fncom.2014.00061

Kamiński, M., \& Blinowska, K. J. (1991). A new method of the description of the information flow in the brain structures. Biological Cybernetics, 65(3), 203-210. doi:10.1007/BF00198091

Katzir, T., Kim, Y.-S. Y.-S., Wolf, M., Morris, R., \& Lovett, M. W. (2008). The Varieties of Pathways to Dysfluent Reading: Comparing Subtypes of Children With Dyslexia at Letter, Word, and Connected Text Levels of Reading. Journal of Learning Disabilities, 41(1), 47-66. doi:10.1177/0022219407311325

Koessler, L., Maillard, L., Benhadid, A., Vignal, J. P., Felblinger, J., Vespignani, H., \& Braun, M. (2009). Automated cortical projection of EEG sensors: anatomical correlation via the international 10-10 system. NeuroImage, 46(1), 64-72.

doi:10.1016/j.neuroimage.2009.02.006

Kujala, J., Pammer, K., Cornelissen, P., Roebroeck, A., Formisano, E., \& Salmelin, R. (2007). Phase coupling in a cerebro-cerebellar network at $8-13 \mathrm{~Hz}$ during reading. Cerebral Cortex, 17(6), 1476-1485. doi:10.1093/cercor/bhl059

Kuś, R., Ginter, J. S., \& Blinowska, K. J. (2006). Propagation of EEG activity during finger movement and its imagination. Acta Neurobiologiae Experimentalis, 66(3), 195-206. Retrieved from http://www.ncbi.nlm.nih.gov/pubmed/17133951

Laszlo, S., \& Federmeier, K. D. (2014). Never Seem to Find the Time: Evaluating the Physiological Time Course of Visual Word Recognition with Regression Analysis of Single Item ERPs. Language and Cognitive Processes, 29(5), 642-661. doi:10.1080/01690965.2013.866259

Leinonen, S., Müller, K., Leppänen, P. H. T., Aro, M., Ahonen, T., \& Lyytinen, H. (2001). Heterogeneity in adult dyslexic readers : Relating processing skills to the speed and accuracy of oral text reading *. Reading and Writing, 14(3-4), 265-296. doi:10.1023/A:1011117620895

Leisman, G. (2002). Coherence of hemispheric function in developmental dyslexia. Brain and Cognition, 48(2-3), 425-431.

Ligges, C., Ungureanu, M., Ligges, M., Blanz, B., \& Witte, H. (2010). Understanding the time variant connectivity of the language network in developmental dyslexia: New insights using Granger causality. Journal of Neural Transmission, 117(4), 529-543. doi:10.1007/s00702-010-0367-x

Linkersdörfer, J., Lonnemann, J., Lindberg, S., Hasselhorn, M., \& Fiebach, C. J. (2012). Grey matter alterations co-localize with functional abnormalities in developmental dyslexia: An ALE meta-analysis. PLoS ONE, 7(8). doi:10.1371/journal.pone.0043122 
Lyon, G. R., Shaywitz, S. E., \& Shaywitz, B. A. (2003). A definition of dyslexia. Annals of Dyslexia, 53(1), 1-14. doi:10.1007/s11881-003-0001-9

Marosi, E., Harmony, T., Becker, J., Reyes, a, Bernal, J., Fernández, T., ... Guerrero, V. (1995). Electroencephalographic coherences discriminate between children with different pedagogical evaluation. International Journal of Psychophysiology : Official Journal of the International Organization of Psychophysiology, 19(1), 23-32. doi:10.1016/0167-8760(94)00059$\mathrm{N}$

Mayhew, S. D., Dirckx, S. G., Niazy, R. K., Iannetti, G. D., \& Wise, R. G. (2010). EEG signatures of auditory activity correlate with simultaneously recorded fMRI responses in humans. NeuroImage, 49(1), 849-64. doi:10.1016/j.neuroimage.2009.06.080

McCandliss, B. D., Cohen, L., \& Dehaene, S. (2003). The visual word form area: expertise for reading in the fusiform gyrus. Trends in Cognitive Sciences, 7(7), 293-299. doi:10.1016/S13646613(03)00134-7

McCandliss, B. D., \& Noble, K. G. (2003). The development of reading impairment: a cognitive neuroscience model. Mental Retardation and Developmental Disabilities Research Reviews, 9(3), 196-204. doi:10.1002/mrdd.10080

Milne, R. D., Hamm, J. P., Kirk, I. J., \& Corballis, M. C. (2003). Anterior-posterior beta asymmetries in dyslexia during lexical decisions. Brain and Language, 84(3), 309-317. doi:10.1016/S0093-934X(02)00557-6

Nazari, M. A., Mosanezhad, E., Hashemi, T., \& Jahan, A. (2012). The effectiveness of neurofeedback training on EEG coherence and neuropsychological functions in children with reading disability. Clinical EEG and Neuroscience, 43(4), 315-22. doi:10.1177/1550059412451880

Oostenveld, R., Fries, P., Maris, E., \& Schoffelen, J. M. (2011). FieldTrip: Open source software for advanced analysis of MEG, EEG, and invasive electrophysiological data. Computational Intelligence and Neuroscience, 2011. doi:10.1155/2011/156869

Paulesu, E., Frith, U., Snowling, M., Gallagher, A., Morton, J., Frackowiak, R. S. J., \& Frith, C. D. (1996). Is developmental dyslexia a disconnection syndrome? Brain, 119(1), 143-157. doi:10.1093/brain/119.1.143

Pugh, K. R., Mencl, W. E., Jenner, a R., Katz, L., Frost, S. J., Lee, J. R., ... Shaywitz, B. A. (2001). Neurobiological studies of reaing and reading disability. Journal of Communicative Disorders, 34, 479-492.

Pugh, K. R., Mencl, W. E., Shaywitz, B. A., Shaywitz, S. E., Fulbright, R. K., Constable, R. T., ... Gore, J. C. (2000). The angular gyrus in developmental dyslexia: task-specific differences in functional connectivity within posterior cortex. Psychological Science: A Journal of the American Psychological Society / APS, 11(1), 51-56. doi:10.1111/1467-9280.00214

Richards, T. L., \& Berninger, V. W. (2008). Abnormal fMRI connectivity in children with dyslexia during a phoneme task: Before but not after treatment. Journal of Neurolinguistics, 21, 294-304. doi:10.1016/j.jneuroling.2007.07.002 


\section{Reading network connectivity and dysfluency}

Richlan, F., Kronbichler, M., \& Wimmer, H. (2009). Functional abnormalities in the dyslexic brain: A quantitative meta-analysis of neuroimaging studies. Human Brain Mapping, 30(10), 3299-3308. doi:10.1002/hbm.20752

Richlan, F., Kronbichler, M., \& Wimmer, H. (2013). Structural abnormalities in the dyslexic brain: A meta-analysis of voxel-based morphometry studies. Human Brain Mapping, 34(11), 3055-3065. doi:10.1002/hbm.22127

Rossell, S. L., Price, C. J., \& Nobre, A. C. (2003). The anatomy and time course of semantic priming investigated by fMRI and ERPs. Neuropsychologia, 41(5), 550-564. doi:10.1016/S0028-3932(02)00181-1

Sandak, R., Mencl, W. E., Frost, S. J., \& Pugh, K. R. (2004). The Neurobiological Basis of Skilled and Impaired Reading: Recent Findings and New Directions. Scientific Studies of Reading, 8(3), 273-292. doi:10.1207/s1532799xssr0803_6

Schaerlakens, A., Kohnstamm, D., \& Lejaegere, M. (1999). Streeflijst woordenschat voor zesjarigen. Gebaseerd op nieun onderzoek in Nederland en België. Lisse: Swets \& Zeitlinger.

Schlögl, A., \& Supp, G. (2006). Analyzing event-related EEG data with multivariate autoregressive parameters. Progress in Brain Research, 159, 135-47. doi:10.1016/S00796123(06)59009-0

Schumacher, E. M., Stiris, T. A., \& Larsson, P. G. (2015). Effective connectivity in long-term EEG monitoring in preterm infants. Clinical Neurophysiology: Official Journal of the International Federation of Clinical Neurophysiology. doi:10.1016/j.clinph.2015.01.017

Schurz, M., Wimmer, H., Richlan, F., Ludersdorfer, P., Klackl, J., \& Kronbichler, M. (2014). Resting-State and Task-Based Functional Brain Connectivity in Developmental Dyslexia. Cerebral Cortex (New York, N.Y. : 1991), 1-13. doi:10.1093/cercor/bhu184

Schwarz, G. (1978). Estimating the Dimension of a Model. The Annals of Statistics, 6(2), 461-464. Retrieved from http://projecteuclid.org/euclid.aos/1176344136

Seth, A. K. (2010). A MATLAB toolbox for Granger causal connectivity analysis. Journal of Neuroscience Methods, 186(2), 262-73. doi:10.1016/j.jneumeth.2009.11.020

Share, D. L. (1995). Phonological recoding and self-teaching: sine qua non of reading acquisition. Cognition, 55(2), 151-218; discussion 219-26. Retrieved from http://www.ncbi.nlm.nih.gov/pubmed/7789090

Shaywitz, S. E., Morris, R., \& Shaywitz, B. A. (2008). The education of dyslexic children from childhood to young adulthood. Annual Review of Psychology, 59, 451-75. doi:10.1146/annurev.psych.59.103006.093633

Simos, P. G., Breier, J. I., Fletcher, J. M., Foorman, B. R., Castillo, E. M., \& Papanicolaou, A. C. (2002). Brain mechanisms for reading words and pseudowords: an integrated approach. Cerebral Cortex (New York, N.Y. : 1991), 12, 297-305. doi:10.1093/cercor/12.3.297

Stokić, M., Milosavljević, Z., \& Subotić, M. (2011). Specific Features of Brain Connectivity 
During Silent Reading in Children With Developmental Dyslexia. Specijalna Edukacija I Rehabilitacija, 10(3), 479-492. Retrieved from

http://scindeks.ceon.rs/article.aspx?artid=1452-73671103479S\&lang=en

van Atteveldt, N., \& Ansari, D. (2014). How symbols transform brain function: A review in memory of Leo Blomert. Trends in Neuroscience and Education, 1-5.

doi:10.1016/j.tine.2014.04.001

van der Mark, S., Klaver, P., Bucher, K., Maurer, U., Schulz, E., Brem, S., ... Brandeis, D. (2011). The left occipitotemporal system in reading: Disruption of focal fMRI connectivity to left inferior frontal and inferior parietal language areas in children with dyslexia. NeuroImage, 54(3), 2426-2436. doi:10.1016/j.neuroimage.2010.10.002

Vandermosten, M., Boets, B., Wouters, J., \& Ghesquière, P. (2012). A qualitative and quantitative review of diffusion tensor imaging studies in reading and dyslexia. Neuroscience and Biobehavioral Reviews, 36(6), 1532-1552. doi:10.1016/j.neubiorev.2012.04.002

Varela, F., Lachaux, J. P., Rodriguez, E., \& Martinerie, J. (2001). The brainweb: phase synchronization and large-scale integration. Nature Reviews. Neuroscience, 2(4), 229-239. doi:10.1038/35067550

Vidal, J. R., Freyermuth, S., Jerbi, K., Hamame, C. M., Ossandon, T., Bertrand, O., ... Lachaux, J.-P. (2012). Long-Distance Amplitude Correlations in the High Gamma Band Reveal Segregation and Integration within the Reading Network. Journal of Neuroscience, 32(19), 6421-6434. doi:10.1523/JNEUROSCI.4363-11.2012

Wimmer, H., \& Schurz, M. (2010). Dyslexia in regular orthographies: manifestation and causation. Dyslexia, 16(4), 283-299. doi:10.1002/dys.411

Wolf, R. C., Sambataro, F., Lohr, C., Steinbrink, C., Martin, C., \& Vasic, N. (2010). Functional brain network abnormalities during verbal working memory performance in adolescents and young adults with dyslexia. Neuropsychologia, 48, 309-318. doi:10.1016/j.neuropsychologia.2009.09.020

Woodhead, Z. V. J., Barnes, G. R., Penny, W., Moran, R., Teki, S., Price, C. J., \& Leff, a P. (2014). Reading Front to Back: MEG Evidence for Early Feedback Effects During Word Recognition. Cerebral Cortex (New York, N.Y. : 1991), 24(3), 817-25. doi:10.1093/cercor/bhs365

Yamada, Y., Stevens, C., Dow, M., Harn, B. A., Chard, D. J., \& Neville, H. J. (2011). Emergence of the neural network for reading in five-year-old beginning readers of different levels of pre-literacy abilities: An fMRI study. NeuroImage, 57(3), 704-713. doi:10.1016/j.neuroimage.2010.10.057

Žarić, G., Fraga González, G., Tijms, J., van der Molen, M. W., Blomert, L., \& Bonte, M. (2014). Reduced neural integration of letters and speech sounds in dyslexic children scales with individual differences in reading fluency. PloS One, 9(10), e110337. doi:10.1371/journal.pone.0110337 


\section{Reading network connectivity and dysfluency}

Žarić, G., Fraga González, G., Tijms, J., van der Molen, M. W., Blomert, L., \& Bonte, M. (2015). Crossmodal deficit in dyslexic children: practice affects the neural timing of letter-speech sound integration. Frontiers in Human Neuroscience, 369. doi:10.3389/fnhum.2015.00369 


\section{CHAPTER 5}

\section{Altered white matter connectivity in 8-10 year old dyslexic and typically reading children}

Manuscript in preparation:

Žarić, G., Timmers, I., Gerretsen P., Fraga González, G., Tijms, J., Van der Molen, M., Blomert, L., Bonte, M. Altered white matter connectivity in 8-10 year old dyslexic and typically reading children 


\begin{abstract}
Reading is a complex cognitive skill subserved by a distributed network of brain regions involved in visual, spoken language and cognitive functions. Atypical structural properties of white matter bundles connecting these regions has been associated with failing reading acquisition in developmental dyslexia. So far, most studies have compared white matter connectivity in dyslexic and typically reading adults with English as their primary language. Here we aim to extend these findings by investigating functional anisotropy (FA) values of white matter bundles in Dutch nine year old dyslexic $(n=21)$ and typically reading $(n=15)$ children. Our results show higher FA values in the right thalamic radiation in dyslexic as compared to typical readers. Interestingly, across groups higher FA values in this tract were related to more dysfluent reading scores. Furthermore, higher FA values along the bilateral thalamic radiations were related to slower rapid automatized naming. On the other hand, typical readers showed a tendency towards higher FA values along multiple tracts in the reading network, and especially in the right uncinated fasciculus which also scaled with better reading and phoneme deletion accuracy. Although speculative at this point, enhanced FA values in the right thalamic radiation might relate to increased and/or more effortful engagement of multisensory processes in dyslexic readers. Furthermore, our results implicate a right hemisphere involvement in the developing reading network.
\end{abstract}




\section{Introduction}

Reading is a complex recent cognitive function, nonetheless mastered by 9095\% of children without notable problems. Conversely, children suffering from developmental dyslexia, a specific reading disability with neurobiological origin and a genetic component (Shaywitz \& Shaywitz, 2005), fail to achieve proficient reading skills despite their normal cognitive abilities and educational opportunities (Blomert, 2005; Lyon, Shaywitz, \& Shaywitz, 2003). A lack of reading fluency has been pointed out as the most severe impairment (Shaywitz, Morris, \& Shaywitz, 2008), particularly in transparent orthographies such as Dutch and German (Wimmer \& Schurz, 2010). Findings of abnormal functional and anatomical white matter connectivity within the neural reading network in dyslexia have been taken to suggest an underlying problem in information transfer within this network (Geschwind, 1965; Paulesu et al., 1996; Wimmer \& Schurz, 2010). So far, most evidence for white matter abnormalities in dyslexia comes from studies comparing groups of adult dyslexic and typical readers of English. Given the dynamic and idiosyncratic nature of reading and white matter development (e.g. Yeatman, Dougherty, Ben-Shachar, \& Wandell, 2012) it is essential to extend these findings to children at different developmental stages as well as to more transparent orthographies in which written and spoken language may be linked in a different way (e.g. Holloway, van Atteveldt, Blomert, \& Ansari, 2013). Here we investigate patterns of white matter connectivity in a group of 8-10-year old dyslexic and typically reading children learning to read in a transparent orthography (Dutch).

As a phylogenetically recent cognitive function reading has been proposed to build on left lateralized neuronal networks for visual object recognition (Schlaggar \& McCandliss, 2007) and spoken language (Liberman, Shankweiler, Fischer, \& Carter, 1974; Pugh, Mencl, Jenner, Lee, et al., 2001). These regions are located along the posterior to anterior axis of the brain and include ventral left occipito-temporal, posterior-temporal, dorsal parieto-temporal and left inferior-frontal regions. These areas have each been associated with specific reading related functions. Hence, posterior-temporal and parieto-temporal areas 
have been shown to play a part in phonological processing (Pugh, Mencl, Jenner, Katz, et al., 2001) and multisensory grapheme-phoneme integration (Blomert, 2011; Simos et al., 2002; van Atteveldt \& Ansari, 2014), while the ventral occipito-temporal region is involved in fast visual word recognition (Jobard, Crivello, \& Tzourio-Mazoyer, 2003; McCandliss, Cohen, \& Dehaene, 2003) and left inferior-frontal regions partake in speech production and word phonological recoding (Sandak, Mencl, Frost, \& Pugh, 2004). Comparison of dyslexic and typical readers has indicated abnormalities in functional activation (Blomert, 2011; McCandliss \& Noble, 2003; Richlan, Kronbichler, \& Wimmer, 2009) and connectivity (Finn et al., 2014; Schurz et al., 2014; van der Mark et al., 2011; Wolf et al., 2010), at multiple levels of the reading network. Dyslexic adults, for example, may show reduced gray matter in posterior areas of the reading network (Linkersdörfer, Lonnemann, Lindberg, Hasselhorn, \& Fiebach, 2012; Richlan, Kronbichler, \& Wimmer, 2013) and abnormalities in white matter connections in temporo-parietal and frontal areas (Vandermosten, Boets, Wouters, \& Ghesquière, 2012).

Anatomical white matter connectivity patterns in the reading network can be measured noninvasively with diffusion weighted imaging (DWI) and tractography (Beaulieu, 2002). DWI measures diffusion of water in the brain. Diffusion refers to the movement trajectories of water molecules as a result of random motion (Beaulieu, 2002). In an environment without barriers, molecules have no preferred direction of diffusion, which is referred to as isotropic diffusion. Within the brain's neural tissue there are multiple constraints that affect diffusion of both extracellular and intracellular water molecules, for instance, cell membranes, cytoskeleton, and macromolecules (Johansen-Berg \& Behrens, 2009). In gray matter which does not have an oriented fiber structure diffusivity is largely isotropic. On the contrary, in white matter, which consists of myelinated axons running parallel to each other, diffusion is larger along the axons than perpendicular to them. Such diffusion along a preferred direction is called anisotropic diffusion. Anisotropic diffusion in white matter tracts is constrained by myelination and several other properties, such as the axonal membranes and neurofibrils (Beaulieu, 2002). 
The usually reported index of anisotropy is fractional anisotropy (FA), which tells us whether the diffusion in certain direction is larger than the average diffusion in the three-dimensional space, i.e. whether diffusion can be described with an ellipsoid rather than a sphere. An FA value of 0 indicates isotropic diffusion and an FA value of 1 indicates maximal anisotropy. FA values have traditionally been used as an index of white matter integrity that scales with the amount of myelination, as disease-related demyelination leads to significant changes in anisotropy measures (Assaf \& Pasternak, 2008). More recently it has been suggested that major determinants of diffusion can be divided into macroscopic geometric factors, such as fiber direction coherence, and microscopic factors, such as axon properties (e.g. axon caliber), while the amount of myelination has a more modulatory role (Vandermosten, Boets, Wouters, et al., 2012).

Possible functional roles of white matter connectivity have been suggested in studies relating $\mathrm{FA}$ values to offline behavioral measures of language and reading skills. Studies in typically developing children found a positive relation between diffusivity measures in the posterior limb of the left internal capsule and word identification scores (Beaulieu et al., 2005), callosal fibers that connect the temporal lobes and phonological awareness (Dougherty et al., 2007), and a white matter region in the left temporal lobe and word reading (Nagy, Westerberg, \& Klingberg, 2004). Interestingly, FA values in the left arcuate fasciculus (AF), a curved tract connecting the posterior superior temporal gyrus/sulcus (i.e. Wernicke's area) to the inferior frontal gyrus (i.e. Broca's area) (Catani, Jones, \& Ffytche, 2005) have been found to be related to both functional activity of the posterior superior temporal sulcus (pSTS) for cross-modal rhyme judgments and behavioral response accuracy, both independently of the age of the participants (Gullick \& Booth, 2014).

Studies comparing white matter between adult typical and impaired readers were conducted mostly with English speaking, but also Swedish, Brazilian Portuguese, Italian, German and Flemish/Dutch. The findings of these studies include abnormal FA values in dyslexic readers in parieto-temporal regions 
including superior longitudinal fasciculus and specifically arcuate fasciculus (Frye et al., 2011; Richards et al., 2008; Steinbrink et al., 2008; Thiebaut De Schotten, Cohen, Amemiya, Braga, \& Dehaene, 2014; Vandermosten, Poelmans, Sunaert, Ghesquière, \& Wouters, 2013; Vandermosten, Boets, Poelmans, et al., 2012), anterior and posterior limb of the left internal capsule and corona radiata (Klingberg et al., 2000; Richards et al., 2008) connecting the thalamus to the cerebral cortex. Further differences were found in corpus callosum, including body, splenium and isthmus (Frye et al., 2008; Richards et al., 2008; Welcome \& Joanisse, 2014), left inferior longitudinal fasciculus (connecting the ventral occipital to the anterior temporal lobe) and inferior fronto-occipital fasciculus (connecting the ventral occipital lobe and the orbitofrontal cortex) (Lebel et al., 2013; Marino et al., 2014; Rimrodt, Peterson, Denckla, Kaufmann, \& Cutting, 2010). Most of these studies also report significant relations between diffusion weighted measures and diverse reading related skills.

Similar differences to those in adult groups were found between dyslexic and typically reading English children in the arcuate and superior longitudinal fasciculus (Carter et al., 2009; Deutsch et al., 2005; Hoeft et al., 2011; Rimrodt et al., 2010; Saygin et al., 2013; Yeatman, Dougherty, Ben-Shachar, et al., 2012; Yeatman et al., 2011), left internal capsule and corona radiata (Niogi \& McCandliss, 2006; Odegard, Farris, Ring, McColl, \& Black, 2009), the corpus callosum (Hasan et al., 2012; Odegard et al., 2009), and the left inferior longitudinal fasciculus and inferior fronto-occipital fasciculus (Yeatman, Dougherty, Ben-Shachar, et al., 2012). On the other hand, the few studies in more transparent orthographies, i.e. Swedish and German, included participants with a wide age range and did not include a group of dyslexic children (Darki, Peyrard-Janvid, Matsson, Kere, \& Klingberg, 2012; Gebauer et al., 2012; Nagy et al., 2004). A study in pre-reading Flemish (Dutch) children yielded differences between children at familial risk of dyslexia and children without the risk in the left inferior fronto-occipital fasciculus, while FA values of the left arcuate and bilateral inferior fronto-occipital fasciculi were related to phonological awareness (Vandermosten et al., 2015). 
An important variable to take into account in research of (a)typical reading development is the dynamic nature of functional brain development. Thus, some studies suggest that FA and other diffusivity measures of white matter tracts follow an exponential pattern of development, with a different maturational time courses for different tracts and slowest progress for frontotemporal tracts (Lebel, Walker, Leemans, Phillips, \& Beaulieu, 2008). Similarly, age related variable white matter development was found in a group of typically developing toddlers (Johnson et al., 2014). Other studies have suggested that the relation between reading ability and diffusion measures correlates with agerelated changes. For example, in one study a significant relation of reading skills and FA values in the left temporal lobe disappeared when controlled for age in children and adolescents (age range 7.8-18.5 years) (Nagy et al., 2004). The role of individual differences in white matter development was highlighted in a recent longitudinal study in 7-15 year old children with variable levels of reading fluency. Yeatman and colleagues (2012) found different patterns of FA maturation in the left arcuate and left inferior longitudinal fasciculus of poor and above-average reading children with poor readers having initially higher FA values that decreased over period of 3 years, and above-average readers initially having low FA values that increased over time (Yeatman, Dougherty, BenShachar, et al., 2012). Interestingly, this model of maturation would imply that at around 10-12 years poor and good readers would show a minimal difference in FA values in these white matter tracts (Yeatman, Dougherty, Ben-Shachar, et al., 2012).

In the current DWI study we investigate structural white matter connectivity in Dutch 9 year old typically reading $(n=15)$ and dyslexic children $(n=21)$. We aimed to test whether the similarities seen among adult readers of different orthographies would also extend to children whose neural reading network is still undergoing dynamic developmental changes (e.g. Yeatman, Dougherty, Ben-Shachar, et al., 2012). In particular, by examining children that learn to read in the transparent Dutch orthography, we aim to extend previous studies investigating the relation of white matter diffusion properties and reading in 
dyslexic and typically reading children that have so far been almost exclusively conducted in the (deep) English orthography.

\section{Methods}

\subsection{Participants}

DWI data of 40 children (24 dyslexic and 16 typical readers) was collected. Children were native Dutch speakers, from Dutch educational groups 4 to 7 (group 4: 3 children, 5: 19, 6: 13, 7:5) having received between 1 and 4 years of reading instruction. Data of one typically reading and three dyslexic children were discarded from further analysis due to the erroneous alignment with T1 weighted images, thus leaving 21 dyslexic and 15 typical readers for the analysis.

Typically reading children were recruited via local schools. Dyslexic children were recruited from the Dutch Regional Institute for Dyslexia (RID), where they were diagnosed as dyslexic following an extensive cognitive psychodiagnostic procedure. The two groups were matched for, age and IQ, $(\geq 85$, Dutch version of Wechsler Intelligence Scale for Children) and were tested on selected subtests (Table 5-1) from the 3DM test battery (Blomert \& Vaessen, 2009). The included subtest were a computerized reading test (high-frequency, low-frequency and pseudowords), phoneme-deletion task, spelling and a letterspeech sound discrimination task (Blomert \& Vaessen, 2009). The scores of total reading accuracy (a ratio of correctly read words and all read words) and fluency (correctly read words in 1.5 minutes) were derived from the reading test. Children were classified as dyslexic if their standard reading score was within the lower 10th percentile. The approval for the research was obtained from the local ethical committee of the Faculty of Psychology and Neuroscience, Maastricht University. 


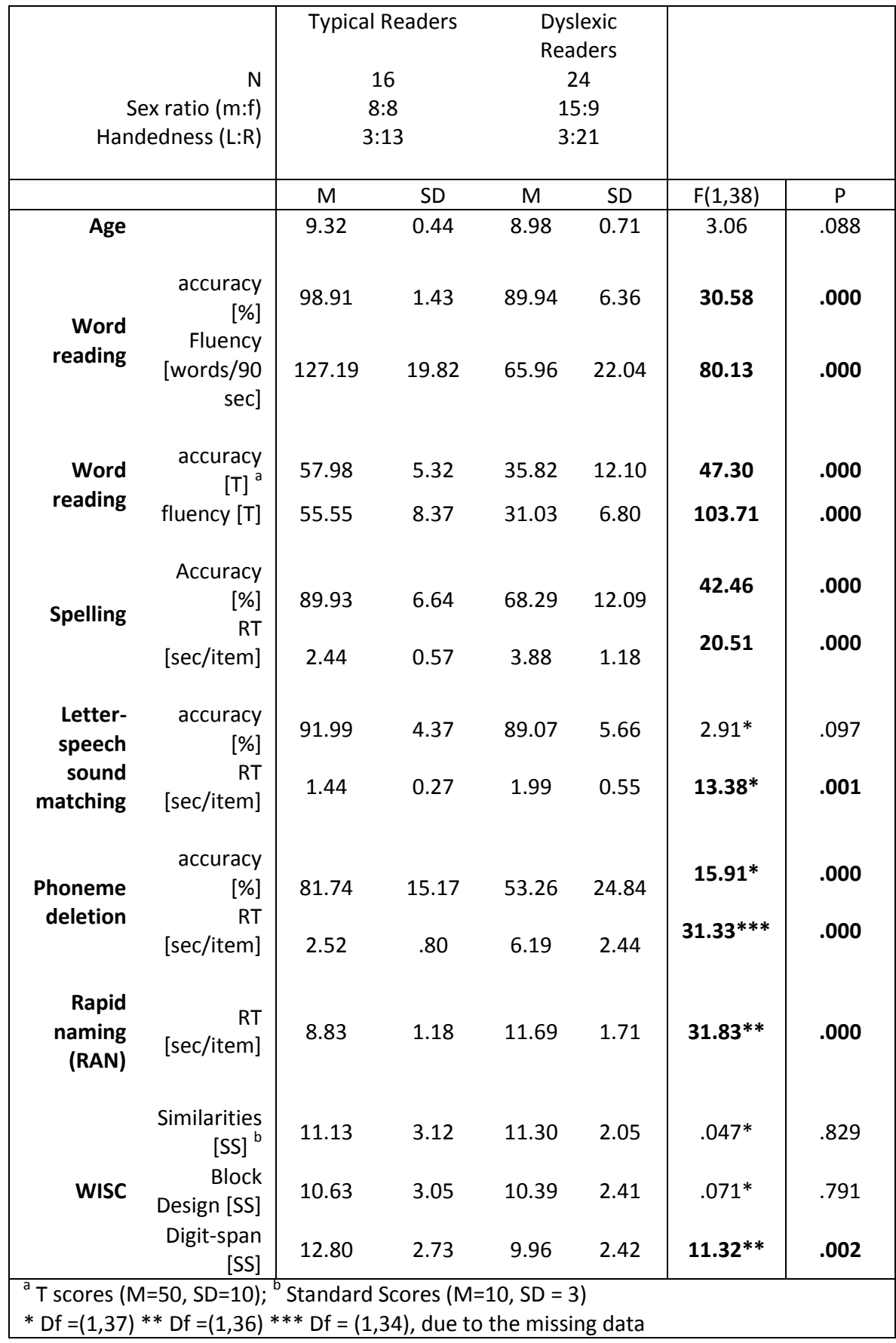

Table 5-1. Behavioral reading scores: Descriptive data and statistical group comparisons for typical and dyslexic readers 


\subsection{DWI data acquisition}

DWI data was acquired using a Siemens 3T MAGNETOM Allegra MR scanner equipped with a high slew-rate head gradient-coil (amplitude $40 \mathrm{mT} / \mathrm{m}$, slew rate $400 \mathrm{~T} / \mathrm{m} / \mathrm{s}$ ) and an 8-channel phased-array head RF-coil. The DWI data were obtained using a doubly refocused spin echo EPI sequence. Due to a scanner update, two different but comparable protocols were used. In the first protocol (typical readers $\mathrm{n}=9$; dyslexic $\mathrm{n}=11$ ), 85 slices [voxel-size $1.8 \mathrm{~mm}$ isotropic; repetition time $[\mathrm{TR}]=10800 \mathrm{~ms}$; echo time [TE] $=84 \mathrm{~ms}$ ) were acquired at b-values of $1000 \mathrm{~s} / \mathrm{mm}^{2}$, with 72 diffusion-encoding gradient directions. In addition, three $\mathrm{b}=0 \mathrm{~s} / \mathrm{mm}^{2}$ images were collected. Total acquisition time for the sequence was 14.05 minutes. In the second protocol (typical readers $n=7$; dyslexic $n=13$ ), 85 slices [voxel-size $1.8 \mathrm{~mm}$ isotropic; repetition time $[\mathrm{TR}]=11000 \mathrm{~ms}$; echo time $[\mathrm{TE}]=85 \mathrm{~ms}$ ) were acquired at bvalues of $1000 \mathrm{~s} / \mathrm{mm}^{2}$, with 72 diffusion-encoding gradient directions. In addition, eight unweighted $\left(\mathrm{b}=0 \mathrm{~s} / \mathrm{mm}^{2}\right.$ ) images were collected. Total acquisition time for the sequence was 14.51 minutes. In addition to the DWI measurement, a high resolution T1-weighted 3D MPRAGE scan (TR $=2250$, $\mathrm{TE}=2.6 \mathrm{~ms}$, flip angle $=9^{\circ}, 256 \times 256$ matrix, 192 sagittal slices, $1 \times 1 \times 1$ $\mathrm{mm}$ voxels) was acquired for gray/white matter boundary segmentation. The subject's head was immobilized using foam cushions.

\subsection{Diffusion data preprocessing}

Pre-processing of the data was performed using mrDiffusion, a part of an open-source mrVista package from Stanford VISTA Lab (available for download from https://github.com/vistalab), and SPM8 working under MATLAB 2014a (The MathWorks, Natick, MA). Anterior and posterior commissures (AC and PC) were manually identified in the T1 weighted anatomical images of each subject and the anatomical images were rotated to the AC-PC plane. The preprocessing of the diffusion data included estimation and correction of eddy current-induced distortions and subject motion by a non-liner co-registration (14 parameters) of an expected distortion based on the phase-encode direction of the acquired data (Rohde, Barnett, Basser, Marenco, \& Pierpaoli, 2004). Motion-corrected unweighted images were averaged and 
aligned to the T1 weighted image. Diffusion-weighted images were individually registered to the obtained unweighted average by a two-stage coarse-to-fine approach. All diffusion weighted images were resampled to $2-\mathrm{mm}$ isotropic voxels using a 7th-order b-spline algorithm from SPM8 (Friston \& Ashburner, 2004), as implemented in mrDiffusion, and eddy-current intensity correction was applied. The diffusion-weighting gradient directions were rotated using the resulting rotation component from the previous step. A robust least-squares algorithm was applied to fit the diffusion tensors and at the same time remove outliers from the tensor estimation step (Chang, Jones, \& Pierpaoli, 2005). The tensors were decomposed to the eigenvalues that were used to compute the fractional anisotropy (FA) (Basser \& Pierpaoli, 1996).

\subsection{Fiber tracking and quantification}

Fiber tracking and quantification was performed using Automated Fiber Quantification package - AFQ (Yeatman, Dougherty, Myall, Wandell, \& Feldman, 2012) for MATLAB. AFQ identifies 20 fiber tracts per subject using a three-step procedure combining deterministic tractography with a streamlines tracking algorithm (STT) (Basser, Pajevic, Pierpaoli, Duda, \& Aldroubi, 2000; Mori, Crain, Chacko, \& Van Zijl, 1999), fiber tract segmentation based on waypoint regions of interest (Wakana et al., 2007) and fiber tract refinement based on a probabilistic fiber tract atlas (Hua et al., 2008).

For whole brain tractography a white matter mask (all voxels with FA>0.3) was used to seed a fourth-order Runge-Kutta path integration method with a stepsize of $1-\mathrm{mm}$. The stopping criteria for streamlines were FA $<0.2$ at the current position and the minimum angle between two consecutive segments greater than $30^{\circ}$. In a next step, fibers were segmented as potentially belonging to the one of 20 fiber groups if they passed through the regions defining the trajectory of the fascicle (waypoint regions of interest - ROI). ROIs were positioned to cut of the central portion of the tract, as it contains more coherent bundles of fibers than the end portions where fibers start to separate towards cortex. Finally, fibers were compared to fiber tract probability maps (Hua et al., 2008) and discarded if they passed through regions of low probability. 
To correct for possible errors in tractography (due to noise, complex fiber orientation and ambiguous stopping criteria) the fibers that were more than 4 standard deviations longer than the mean fiber length across subjects, or deviated more than 5 standard deviations from the core of the fiber tract were removed in the iterative process until there were no more outliers. Finally, fibers of each tract were resampled to 100 equal nodes and fractional anisotropy (FA) was calculated at each node using spline interpolation of the diffusion properties. The FA value of the tract at each node was calculated as a weighted average of the diffusion properties at that node of each fiber (Yeatman, Dougherty, Myall, et al., 2012).

\subsection{Group analysis}

Due to the strict criterion for tract identification implemented in AFQ (Johnson et al., 2014) the number of detected fibers per tract differed across subjects (see Table 5-2 for range) and in some subjects not all the tracts were identified. To avoid spurious effects due to subjects with small(er) number of fibers, we excluded from group analysis per tract (1) subjects in which the current tract was not identified and (2) subjects in which we identified a small number of fibers for the current tract relative to the group ( $<10$ percentile). The number of subjects per tract included in the analysis is given in Table 5-2.

To evaluate group differences between typically reading and dyslexic children, we first averaged FA values of each tract over 100 nodes to obtain one mean FA measure per tract per subject. These mean FA values were statistically analyzed using unpaired t-tests. In case of a significant group difference we also calculated correlations of the mean FA values with age and reading fluency. As age was a significant predictor of raw fluency scores, we computed correlations for both raw and t scores. Furthermore, we performed more in depth group analysis using unpaired t-tests per node per fiber to investigate whether the analysis on mean FA value attenuated more specific differences along the tracts. Furthermore we calculated correlations per node per tract with age, raw and standard scores of reading fluency. To account for the multiple comparisons for both t-tests and correlations obtained across 100 nodes we 
used permutations $(n=1000)$ based correction per tract (Nichols \& Holmes, 2002).

\begin{tabular}{|c|c|c|c|c|}
\hline Tract & $\begin{array}{c}\mathbf{N} \\
\text { subjects }\end{array}$ & $\begin{array}{l}\text { M (SD) } \\
\text { fibers }\end{array}$ & $\begin{array}{l}\text { Range of } \\
\text { fibers }\end{array}$ & $\begin{array}{l}\mathrm{N} \text { (typ:dys) in } \\
\text { analysis }\end{array}$ \\
\hline Left thalamic radiation & 36 & $2567(690)$ & $1075-3924$ & $32(19: 13)$ \\
\hline Right thalamic radiation & 36 & $3103(872)$ & $1371-5385$ & $32(19: 13)$ \\
\hline Left corticospinal & 36 & $\begin{array}{c}5432 \\
(1061)\end{array}$ & $3354-7223$ & $32(18: 14)$ \\
\hline Right corticospinal & 36 & $\begin{array}{c}6409 \\
(1354)\end{array}$ & 2849-9394 & $32(19: 13)$ \\
\hline Left cingulum cingulate & 36 & $954(348)$ & $366-1679$ & $32(18: 14)$ \\
\hline Right cingulum cingulate & 36 & $284(183)$ & $29-731$ & $32(19: 13)$ \\
\hline $\begin{array}{l}\text { Left cingulum } \\
\text { hippocampus }\end{array}$ & 31 & $61(105)$ & $2-576$ & $28(14: 14)$ \\
\hline $\begin{array}{l}\text { Right cingulum } \\
\text { hippocampus }\end{array}$ & 35 & $84(74)$ & $2-358$ & $32(19: 13)$ \\
\hline Forceps major & 36 & 2124 (949) & $365-5434$ & $32(17: 15)$ \\
\hline Forceps minor & 36 & $\begin{array}{c}6180 \\
(1750)\end{array}$ & $2714-10746$ & $32(18: 14)$ \\
\hline $\begin{array}{l}\text { Left inferior fronto- } \\
\text { occipital }\end{array}$ & 36 & $1888(857)$ & $665-4351$ & $32(18: 14)$ \\
\hline $\begin{array}{l}\text { Right inferior fronto- } \\
\text { occipital }\end{array}$ & 36 & $1630(886)$ & $28-3885$ & $32(19: 13)$ \\
\hline Left inferior longitudinal & 36 & 581 (449) & 14-2079 & $32(18: 14)$ \\
\hline $\begin{array}{l}\text { Right inferior } \\
\text { longitudinal }\end{array}$ & 36 & 729 (529) & 9-1962 & $32(19: 13)$ \\
\hline Left superior longitudinal & 36 & 964 (593) & $117-2622$ & $32(18: 14)$ \\
\hline $\begin{array}{l}\text { Right superior } \\
\text { longitudinal }\end{array}$ & 36 & $\begin{array}{c}2680 \\
(1246)\end{array}$ & $656-6848$ & $32(19: 13)$ \\
\hline Left uncinate & 36 & $1122(564)$ & $130-3026$ & $32(20: 12)$ \\
\hline Right uncinate & 36 & $1652(686)$ & $402-3676$ & $32(19: 13)$ \\
\hline Left arcuate & 36 & 1714 (869) & $498-4437$ & $32(19: 13)$ \\
\hline Right arcuate & 35 & $1286(935)$ & $6-3372$ & $32(18: 14)$ \\
\hline
\end{tabular}

Table 5-2. Number of subjects in which the tracts were detected with mean (SD) number and range of detected fibers per tract and number of subjects retained for the analysis after excluding subjects in which small number of fibers was detected ( $<10$ percentile) 


\section{Results}

\subsection{Group differences in mean FA}

The analysis of mean FA values showed a significant group difference for the right thalamic radiation $(\mathrm{t}(30)=2.16, \mathrm{p}=0.039$ ) (Figure $5-1$ ) with higher $\mathrm{FA}$ values for dyslexic children $(\mathrm{FA}=0.486(0.018))$ as compared to controls $(\mathrm{FA}=0.471(0.023))$. Interestingly, the individual mean $\mathrm{FAs}$ of the right thalamic radiation correlated significantly with raw scores of reading fluency $(r=-0.366$, $\mathrm{p}=0.040$, Figure 5-2). Although age did not correlate significantly with the mean FA, it correlated significantly with the raw scores of reading fluency $(\mathrm{r}=0.469, \mathrm{p}=0.002)$. Thus, we further correlated the mean FA values of the right thalamic radiation with age appropriate reading fluency scores ( $\mathrm{T}$ scores) to account for possible confounding effects of the age. This correlation was also significant $(r=-0.377, p=0.033$, Figure $5-2)$. In summary, dyslexics had higher mean fractional anisotropy of the right thalamic radiation and across groups higher FA values related to less fluent reading.
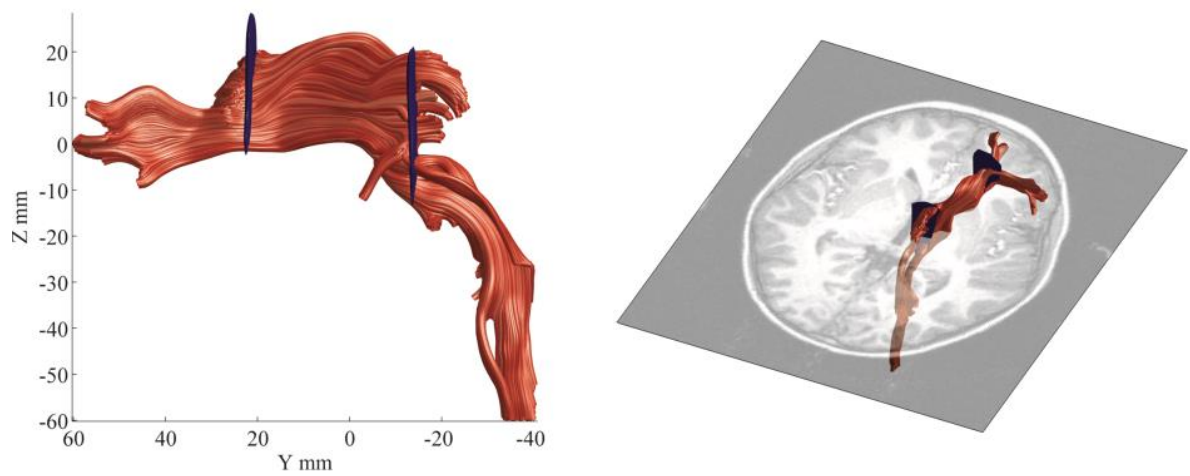

Figure 5-1. Right thalamic radiation (left) and its position relative to t1 image ( $z$ axis=1). Waypoint regions of interest between which the mean FA value was calculated are represented in dark blue.

\subsection{Differences in FA values along the tracts}

The more in depth analysis of group differences in FA values along the tracts did not yield any significant differences when corrected for multiple comparisons. However, uncorrected $\mathrm{p}$ values reached significance in multiple fibers in the right hemisphere (Figure 5-3). At the uncorrected level, dyslexics 
had higher FA values along the right thalamic radiation, lower FA values in the posterior (vertical) part of the arcuate, temporal part of the uncinate and anterior part of the inferior longitudinal fasciculus, as well as in the middle of the right cingulum cingulate. In the left hemisphere, the only (uncorrected) significant result was in the corticospinal tract with dyslexics having lower FA value at the inferior end of the tract and higher FA values at the superior end of the tract (Figure 5-3).
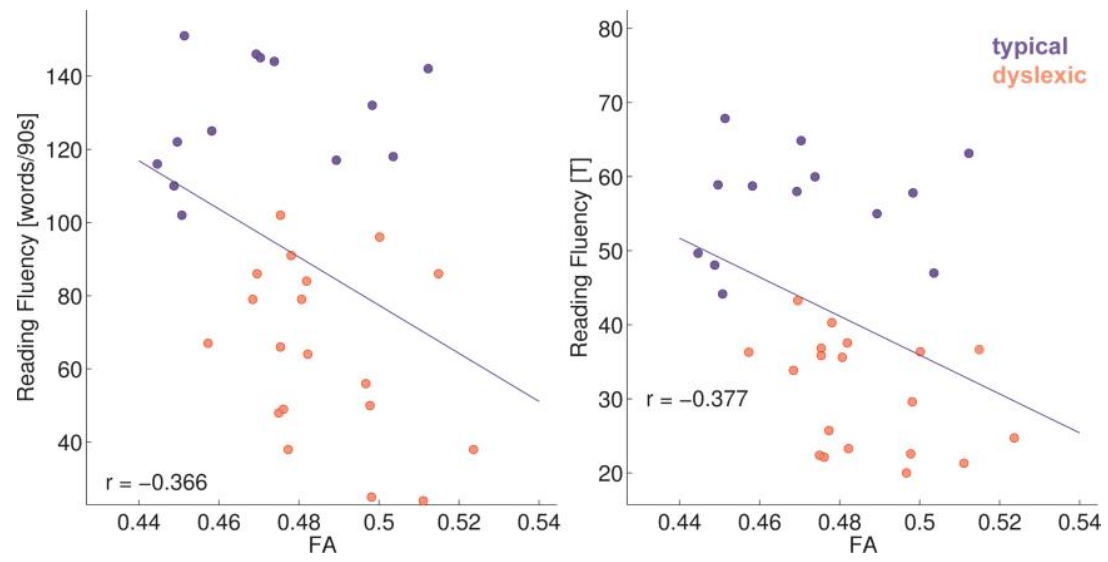

Figure 5-2. Correlations of mean FA value of right thalamic radiation with raw scores (left) and age appropriate T scores (right) of the reading fluency.

Interestingly, the higher FA across right and left thalamic radiation was related to slower rapid automatized naming (Table 5-3). On the contrary, our results indicated that higher age appropriate scores of reading accuracy where related to higher FA values along left arcuate and right uncinate fasciculus. Moreover, the FA values in the right uncinate fasciculus were positively related to accuracy on phoneme deletion task. Next, higher FA values in right cingulum of hippocampus were related to faster phoneme deletion and more accurate letterspeech sound matching. Still, these results should be taken with reserve due to the small number of fibers detected in the hippocampus. Finally, age was negatively related to FA values in the left cingulum cingulate and the left arcuate fasciculus, suggesting a regressive age-related change in these regions. 


\begin{tabular}{|llcc|}
\hline Tract & Behavioral measure & Mean r & Nodes \\
\hline right thalamic radiation & RAN [sec/item] & 0.540 & $68-74$ \\
left thalamic radiation & RAN [sec/item] & 0.535 & $69-75$ \\
& reading accuracy [T score] & 0.506 & $65-67$ \\
left arcuate fasciculus & age [years] & -0.551 & $32-37$ \\
right uncinate & reading accuracy [T score] & 0.547 & $12-25$ \\
fasciculus & phoneme deletion accuracy [\%] & 0.588 & $8-25$ \\
right cingulum & phoneme deletion RT [sec/item] & -0.569 & $61-69$ \\
hippocampus & letter-speech sound matching & 0.548 & $28-30$ \\
left cingulum cingulate & age [years] & -0.566 & $40-44$ \\
\hline
\end{tabular}

Table 5-3. Significant correlations $\left(p_{\text {corr }}<0.05\right)$ between FA values along 100 nodes of the tracts and reading scores and age.

\section{Discussion}

In the present study, we examined differences in structural white-matter connectivity in 8-10 year old typically reading and dyslexic children. To this end we performed deterministic tractography and quantified both the mean FA values for each of 20 white matter tracts and the FA values across hundred equally spaced nodes along each tract. Our results indicate group differences in the right thalamic radiation with dyslexics showing higher mean FA values. This group difference was negatively correlated with reading fluency. The analysis along the tracts yielded only slight differences, predominantly in the right hemisphere, but these did not survive multiple comparison correction.

\subsection{Higher mean FA of the right thalamic radiation scale with reading dysfluency}

We found higher mean FA values in the right thalamic radiation in dyslexic children and the increase in FA values scaled with reading (dys)fluency. Similarly, a recent DTI study investigating thalamo-cortical connectivity in dyslexia found that English-speaking dyslexic children have increased connectivity between thalamus and both the lateral prefrontal cortex and the sensorimotor cortex (Fan, Davis, Anderson, \& Cutting, 2014). Importantly, 
similar to our negative correlations with reading fluency and positive correlations with reaction time on rapid automatized naming, Fan et al., (2014) observed a negative correlation with basic reading scores (composite score of the visual word identification and word attack measures) and thalamo-cortical connectivity. Interestingly, the authors hypothesized that the increased connectivity towards sensory motor cortex could represent "a prolonged multisensory engagement phase" in dyslexic children (Fan et al., 2014). Although speculative at this point, this may be in line with a previous fMRI study showing deviant letter speech-sound integration (Blau et al., 2010) in a subset of the children included in the current study. Furthermore, it may relate to our previous EEG studies in which a different group of 8-9 year old dyslexic children showed reduced neural integration of letters and speech sounds (Žarić et al., 2014, 2015). The impaired crossmodal integration may lead to prolonged letter by letter reading in dyslexic children, instead of shifting to more visual word recognition (Ehri, 2005; Share, 1995). On a more general level, stronger connectivity towards sensory motor cortex would be in line with stronger electrophysiological functional connectivity towards and from central sites that was also observed in 8-9 year old dyslexic as compared to typically reading children (see Chapter 4). Interestingly, FA of the left thalamus in young adult English typical readers has been found to show the opposite effect of a positive correlation with visual word identification scores (Lebel et al., 2013). To further understand the functional significance of these opposite results in children and adults, in future studies it would be essential to follow thalamo-cortical tracts during dyslexic and typical reading development using a longitudinal study design.

Further support for a different functional organization of the thalamo-cortical pathways in dyslexia comes from observations of increased functional connectivity in the right thalamus and other regions during a verbal working memory task (Wolf et al., 2010) and studies finding differences in functional activity of the thalamus between dyslexic and typical readers during reading related tasks (Brunswick, Mccrory, Price, Frith, \& Frith, 1999; Diaz, Hintz, 
Kiebel, \& von Kriegstein, 2012; Georgiewa et al., 1999; Hoeft et al., 2007; Pugh et al., 2008).
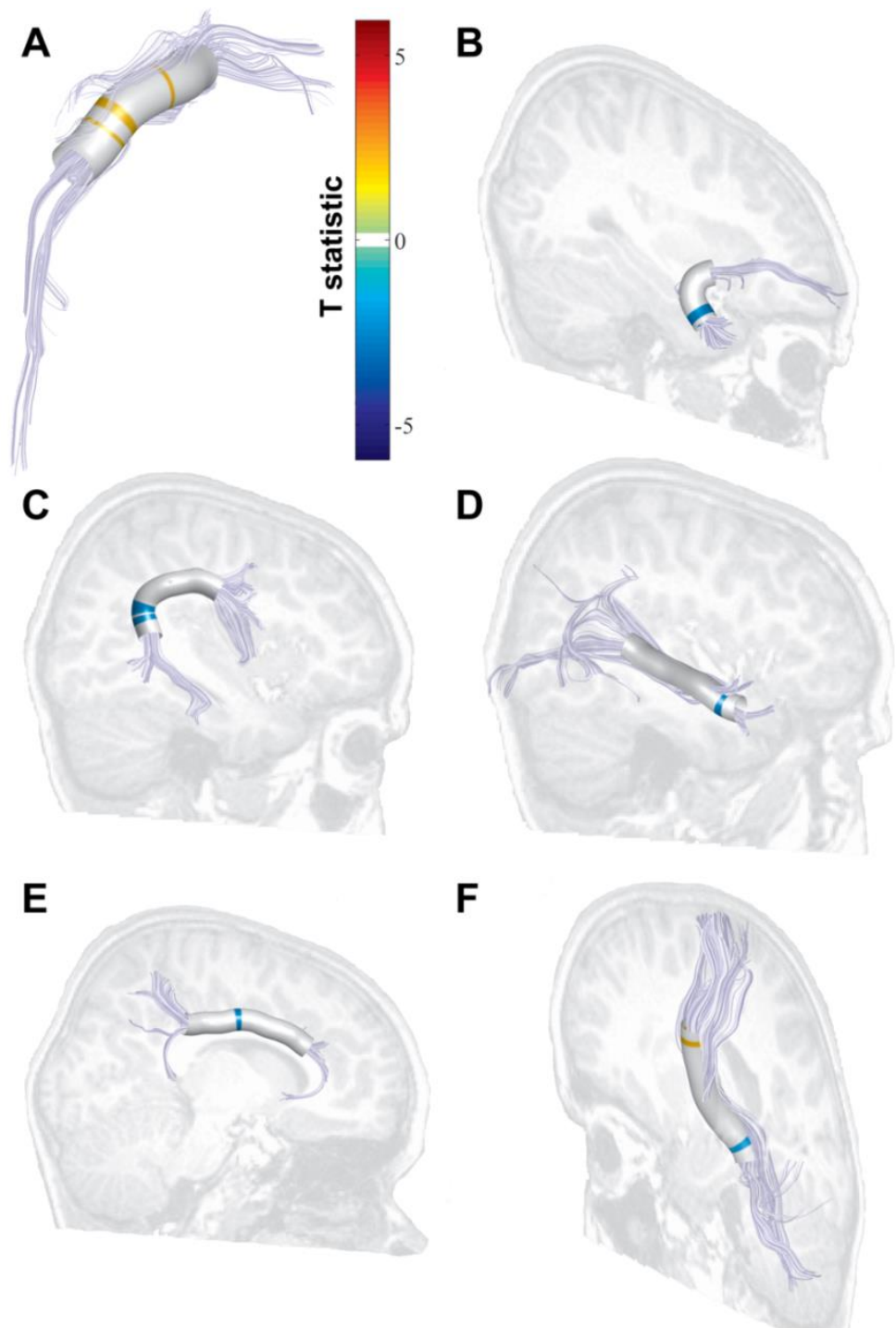

Figure 5-3. Uncorrected significant differences between groups (positive values indicate larger FA values in the dyslexic group; nonsignificant values are set to zero): A. right thalamic radiation; B. right uncinate fasciculus; C. right arcuate fasciculus; D. right inferior longitudinal fasciulus; $E$. right cingulum cingulate; $F$. left cortico-spinal tract 


\subsection{Tendency for higher FA in typical readers along the tracts in the right hemisphere}

At the uncorrected threshold typical readers exhibited higher FA values along multiple tracts implicated in the reading, albeit in the right hemisphere only. These FA values showed significant correlations with reading related scores, and may therefore suggest a reduced lateralization of reading functions to the left hemisphere. In particular these findings may suggest a slightly higher involvement of the right uncinate fasciculus in the 8-10 year old typical readers. However, this finding should be taken with caution at this point as these differences were no longer significant after correction for multiple comparisons.

Our findings extend previous observations of the absence of a left hemisphere specialization in the earlier stages of reading. Interestingly, a recent DWI study in pre-reading Dutch children also did not find strong evidence for the a priori a priori left-hemisphere specialization before reading acquisition as they found relation between bilateral ventral connectivity and phonological awareness (Vandermosten et al., 2015). Furthermore, a study investigating functional activation during reading related tasks found that the left hemisphere specializes over the years (Turkeltaub, Gareau, Flowers, Zeffiro, \& Eden, 2003). Thus our 8-10 year old children may still rely on both hemispheres for reading. Furthermore, as we found correlations of FA values with age exclusively in the left hemisphere, ongoing maturation of the left hemisphere could attenuate differences between the groups (Yeatman, Dougherty, BenShachar, et al., 2012).

Our results further support the role of the (right) uncinate fasciculus as a part of the ventral-reading stream. There was a tendency for higher FA values in typical readers in right uncinate fasciculus, and these FA values were related to reading accuracy and phoneme deletion accuracy. Uncinate fasciculus connects the anterior temporal lobe to the orbitofrontal cortex (Hasan et al., 2009). Interestingly, a recent study in adult English typical readers found relations of diffusion measures of left uncinate fasciculus with the speed of reading 
exception and regular words. Similar to our study, there were no relations of behavioral measures to the FA values of the inferior fronto-occipital, inferior longitudinal and arcuate fasciculus (Cummine et al., 2013). Moreover, the integrity (mean diffusivity) of the right uncinate fasciculus was related to the speed of reading in both mono- and bilinguals, while the integrity of the right arcuate and bilateral superior longitudinal fasciculi were related to reading only in bilinguals, further emphasizing the role of the right hemisphere in reading (Bakhtiari, Boliek, \& Cummine, 2014). Moreover, FA values of both right (Odegard et al., 2009) and left (Welcome \& Joanisse, 2014) uncinate fasciculi have been related to the phonemic decoding.

Although we also found a relation between higher FA values in the right cingulum of the hippocampus and faster phoneme deletion and more accurate letter speech sound matching, these findings need to be taken with caution due to the overall small number of fibers detected in this area. Nevertheless, the role of hippocampus in reading was indicated before as the gray matter volume of the right hippocampus (among other areas) increased after reading training (Krafnick, Flowers, Napoliello, \& Eden, 2011).

\subsection{Limitations and future directions}

Although the present analysis shows overall group differences in the right thalamic radiation, the more fine-grained analysis along the tracts did not yield significant effects when correcting for multiple comparisons. Because Automated Fiber Quantification (AFQ) package uses deterministic tractography and strict criterions for tract identification in some subjects not all the tracts were identified or they had relatively small number of fibers Johnson et al., 2013). At this point we excluded these subjects from the analysis, but it would be interesting to see if the usage of probabilistic tractography would help in identification of all the tracts in all the subjects, thus not having to exclude subjects from the analysis. Furthermore, at this point we restricted analysis only to a portion of tracts between the waypoint ROIs as the fiber bundles are most consistent in those regions, but this could attenuate group differences. Thus, it would be interesting to analyze the whole tract profiles, as AFQ allows for this 
option after additional coregistration procedures (Yeatman, Dougherty, Myall, et al., 2012). Beyond further analysis based on probabilistic tractography and improved co-registration, another interesting possibility would be to analyze white matter organization in separate groups of dyslexic readers based on the severity of their reading dysfluency (Žarić et al., 2014, 2015). Finally, as reading training can influence both gray and white matter plasticity (Keller \& Just, 2009; Krafnick et al., 2011) it would be of interest to investigate possible effects of the training aiming to enhance letter-speech sound integration on the white matter properties.

\section{Acknowledgements}

We would like to thank our colleagues involved in data collection: Alard Roebroek, Nienke van Atteveldt, Vera Blau, Jochen Seitz, Joel Reithler, Dries Froyen, Anke Ley. We would also like to express our gratitude to Anniek Vaessen for her help with the behavioral analysis and to Matteo Bastiani and Jason Yeatman for their advice on DTI preprocessing.

\section{References}

Assaf, Y., \& Pasternak, O. (2008). Diffusion tensor imaging (DTI)-based white matter mapping in brain research: A review. Journal of Molecular Neuroscience, 34, 51-61. doi:10.1007/s12031007-0029-0

Bakhtiari, R., Boliek, C., \& Cummine, J. (2014). Investigating the contribution of ventral-lexical and dorsal-sublexical pathways during reading in bilinguals. Frontiers in Human Neuroscience, 8(July), 507. doi:10.3389/fnhum.2014.00507

Basser, P. J., Pajevic, S., Pierpaoli, C., Duda, J., \& Aldroubi, A. (2000). In vivo fiber tractography using DT-MRI data. Magnetic Resonance in Medicine, 44(4), 625-632. doi:10.1002/15222594(200010)44:4<625::AID-MRM17>3.0.CO;2-O

Basser, P. J., \& Pierpaoli, C. (1996). Microstructural and physiological features of tissues elucidated by quantitative-diffusion-tensor MRI. Journal of Magnetic Resonance. Series B, 111(3), 209-19. Retrieved from http://www.ncbi.nlm.nih.gov/pubmed/8661285

Beaulieu, C. (2002). The basis of anisotropic water diffusion in the nervous system - A technical review. NMR in Biomedicine, 15, 435-455. doi:10.1002/nbm.782 


\section{Altered structural connectivity in dyslexia}

Beaulieu, C., Plewes, C., Paulson, L. A., Roy, D., Snook, L., Concha, L., \& Phillips, L. (2005). Imaging brain connectivity in children with diverse reading ability. NeuroImage, 25, 12661271. doi:10.1016/j.neuroimage.2004.12.053

Blau, V., Reithler, J., van Atteveldt, N., Seitz, J., Gerretsen, P., Goebel, R., \& Blomert, L. (2010). Deviant processing of letters and speech sounds as proximate cause of reading failure: a functional magnetic resonance imaging study of dyslexic children. Brain: A Journal of Neurology, 133(Pt 3), 868-79. doi:10.1093/brain/awp308

Blomert, L. (2005). Dyslexie in Nederland. Amsterdam: Uitgeverij Nieuwezijds. Amsterdam: Uitgeverij Nieuwezijds. Retrieved from http://www.boomtestuitgevers.nl/upload/Dyslexie_in_Nederland_Leo_Blomert.pdf

Blomert, L. (2011). The neural signature of orthographic-phonological binding in successful and failing reading development. NeuroImage, 57(3), 695-703. doi:10.1016/j.neuroimage.2010.11.003

Blomert, L., \& Vaessen, A. A. (2009). 3DM Differential Diagnostiek van Dyslexie: Cognitieve analyse van lezen en spellen (Vol. 10). Amsterdam: Boom test uitgevers.

Brunswick, N., Mccrory, E., Price, C. J., Frith, C. D., \& Frith, U. (1999). Explicit and implicit processing of words and pseudowords by adult developmental dyslexics A search for Wernicke's Wortschatz?, 1901-1917.

Carter, J. C., Lanham, D. C., Cutting, L. E., Clements-Stephens, A. M., Chen, X., Hadzipasic, M., ... Kaufmann, W. E. (2009). A dual DTI approach to analyzing white matter in children with dyslexia. Psychiatry Research - Neuroimaging, 172(3), 215-219. doi:10.1016/j.pscychresns.2008.09.005

Catani, M., Jones, D. K., \& Ffytche, D. H. (2005). Perisylvian language networks of the human brain. Annals of Neurology, 57, 8-16. doi:10.1002/ana.20319

Chang, L. C., Jones, D. K., \& Pierpaoli, C. (2005). RESTORE: Robust estimation of tensors by outlier rejection. Magnetic Resonance in Medicine, 53(5), 1088-1095. doi:10.1002/mrm.20426

Cummine, J., Dai, W., Borowsky, R., Gould, L., Rollans, C., \& Boliek, C. (2013). Investigating the ventral-lexical, dorsal-sublexical model of basic reading processes using diffusion tensor imaging. Brain Structure and Function, 1-11. doi:10.1007/s00429-013-0666-8

Darki, F., Peyrard-Janvid, M., Matsson, H., Kere, J., \& Klingberg, T. (2012). Three dyslexia susceptibility genes, DYX1C1, DCDC2, and KIAA0319, affect temporo-parietal white matter structure. Biological Psychiatry, 72(8), 671-676. doi:10.1016/j.biopsych.2012.05.008

Deutsch, G. K., Dougherty, R. F., Bammer, R., Siok, W. T., Gabrieli, J. D. E., \& Wandell, B. (2005). Children's reading performance is correlated with white matter structure measured by diffusion tensor imaging. Cortex; a Journal Devoted to the Study of the Nervous System and Behavior, 41, 354-363. doi:10.1016/S0010-9452(08)70272-7 
Diaz, B., Hintz, F., Kiebel, S. J., \& von Kriegstein, K. (2012). Dysfunction of the auditory thalamus in developmental dyslexia. Proceedings of the National Academy of Sciences, 109(34), 13841-13846. doi:10.1073/pnas.1119828109

Dougherty, R. F., Ben-Shachar, M., Deutsch, G. K., Hernandez, A., Fox, G. R., \& Wandell, B. a. (2007). Temporal-callosal pathway diffusivity predicts phonological skills in children. Proceedings of the National Academy of Sciences of the United States of America, 104(20), 85568561. doi:10.1073/pnas.0608961104

Ehri, L. C. (2005). Learning to Read Words: Theory, Findings, and Issues. Scientific Studies of Reading, 9(2), 167-188. doi:10.1207/s1532799xssr0902_4

Fan, Q., Davis, N., Anderson, A. W., \& Cutting, L. E. (2014). Thalamo-cortical connectivity: what can diffusion tractography tell us about reading difficulties in children? Brain Connectivity, 4(6), 428-39. doi:10.1089/brain.2013.0203

Finn, E. S., Shen, X., Holahan, J. M., Scheinost, D., Lacadie, C., Papademetris, X., ... Constable, R. T. (2014). Disruption of functional networks in dyslexia: A whole-brain, data-driven analysis of connectivity. Biological Psycbiatry, 76(5), 397-404.

doi:10.1016/j.biopsych.2013.08.031

Friston, K. J., \& Ashburner, J. (2004). Generative and recognition models for neuroanatomy. NeuroImage, 23(1), 21-24. doi:10.1016/j.neuroimage.2004.04.021

Frye, R. E., Hasan, K., Xue, L., Strickland, D., Malmberg, B., Liederman, J., \& Papanicolaou, A. (2008). Splenium microstructure is related to two dimensions of reading skill. Neuroreport, 19(16), 1627-1631. doi:10.1097/WNR.0b013e328314b8ee

Frye, R. E., Liederman, J., Hasan, K. M., Lincoln, A., Malmberg, B., McLean, J., \& Papanicolaou, A. (2011). Diffusion tensor quantification of the relations between microstructural and macrostructural indices of white matter and reading. Human Brain Mapping, 32, 1220-1235. doi:10.1002/hbm.21103

Gebauer, D., Fink, A., Filippini, N., Johansen-Berg, H., Reishofer, G., Koschutnig, K., ... Enzinger, C. (2012). Differences in integrity of white matter and changes with training in spelling impaired children: a diffusion tensor imaging study. Brain Structure \& Function, 217(3), 747-60. doi:10.1007/s00429-011-0371-4

Georgiewa, P., Rzanny, R., Hopf, J. M., Knab, R., Glauche, V., Kaiser, W. a, \& Blanz, B. (1999). fMRI during word processing in dyslexic and normal reading children. Neuroreport, 10(16), 3459-3465. doi:10.1097/00001756-199911080-00036

Geschwind, N. (1965). Disconnexion syndromes in animals and man. I. Brain, 88(2), 237-294. doi:10.1093/brain/88.2.237

Gullick, M. M., \& Booth, J. R. (2014). Individual Differences in Cross-modal Brain Activity Predict Arcuate Fasciculus Connectivity in Developing Readers. Journal of Cognitive Neuroscience, 1-16. doi:10.1162/jocn_a_00581 


\section{Altered structural connectivity in dyslexia}

Hasan, K. M., Iftikhar, A., Kamali, A., Kramer, L. a., Ashtari, M., Cirino, P. T., ... Ewing-Cobbs, L. (2009). Development and aging of the healthy human brain uncinate fasciculus across the lifespan using diffusion tensor tractography. Brain Research, 1276, 67-76. doi:10.1016/j.brainres.2009.04.025

Hasan, K. M., Molfese, D. L., Walimuni, I. S., Stuebing, K. K., Papanicolaou, A. C., Narayana, P. a., \& Fletcher, J. M. (2012). Diffusion tensor quantification and cognitive correlates of the macrostructure and microstructure of the corpus callosum in typically developing and dyslexic children. NMR in Biomedicine, 25(February), 1263-1270. doi:10.1002/nbm.2797

Hoeft, F., McCandliss, B. D., Black, J. M., Gantman, A., Zakerani, N., Hulme, C., ... Gabrieli, J. D. E. (2011). Neural systems predicting long-term outcome in dyslexia. Proceedings of the National Academy of Sciences of the United States of America, 108(1), 361-6. doi:10.1073/pnas.1008950108

Hoeft, F., Meyler, A., Hernandez, A., Juel, C., Taylor-Hill, H., Martindale, J. L., ... Gabrieli, J. D. E. (2007). Functional and morphometric brain dissociation between dyslexia and reading ability. Proceedings of the National Academy of Sciences of the United States of America, 104(10), 4234-4239. doi:10.1073/pnas.0609399104

Holloway, I. D., van Atteveldt, N., Blomert, L., \& Ansari, D. (2013). Orthographic Dependency in the Neural Correlates of Reading: Evidence from Audiovisual Integration in English Readers. Cerebral Cortex (New York, N.Y. : 1991). doi:10.1093/cercor/bht347

Hua, K., Zhang, J., Wakana, S., Jiang, H., Li, X., Reich, D. S., ... Mori, S. (2008). Tract probability maps in stereotaxic spaces: analyses of white matter anatomy and tract-specific quantification. NeuroImage, 39(1), 336-47. doi:10.1016/j.neuroimage.2007.07.053

Jobard, G., Crivello, F., \& Tzourio-Mazoyer, N. (2003). Evaluation of the dual route theory of reading: A metanalysis of 35 neuroimaging studies. NeuroImage, 20, 693-712. doi:10.1016/S1053-8119(03)00343-4

Johansen-Berg, H., \& Behrens, T. E. J. (2009). Diffusion MRI (First.). Elsevier Inc.

Johnson, R. T., Yeatman, J. D., Wandell, B. a, Buonocore, M. H., Amaral, D. G., \& Nordahl, C. W. (2014). Diffusion properties of major white matter tracts in young, typically developing children. NeuroImage, 88, 143-154. doi:10.1016/j.neuroimage.2013.11.025

Johnson, R. T., Yeatman, J. D., Wandell, B. A., Buonocore, M. H., Amaral, D. G., \& Nordahl, C. W. (2013). Diffusion properties of major white matter tracts in young, typically developing children. NeuroImage, 88C, 143-154. doi:10.1016/j.neuroimage.2013.11.025

Keller, T. a, \& Just, M. A. (2009). Altering cortical connectivity: remediation-induced changes in the white matter of poor readers. Neuron, 64(5), 624-31. doi:10.1016/j.neuron.2009.10.018

Klingberg, T., Hedehus, M., Temple, E., Salz, T., Gabrieli, J. D., Moseley, M. E., \& Poldrack, R. a. (2000). Microstructure of temporo-parietal white matter as a basis for reading ability: 
evidence from diffusion tensor magnetic resonance imaging. Neuron, 25(2), 493-500. Retrieved from http://www.ncbi.nlm.nih.gov/pubmed/10719902

Krafnick, A. J., Flowers, D. L., Napoliello, E. M., \& Eden, G. F. (2011). Gray matter volume changes following reading intervention in dyslexic children. NeuroImage, 57(3), 733-41. doi:10.1016/j.neuroimage.2010.10.062

Lebel, C., Shaywitz, B. A., Holahan, J., Shaywitz, S. E., Marchione, K., \& Beaulieu, C. (2013). Diffusion tensor imaging correlates of reading ability in dysfluent and non-impaired readers. Brain and Language, 125(2), 215-222. doi:10.1016/j.bandl.2012.10.009

Lebel, C., Walker, L., Leemans, A., Phillips, L., \& Beaulieu, C. (2008). Microstructural maturation of the human brain from childhood to adulthood. NeuroImage, 40(3), 1044-55. doi:10.1016/j.neuroimage.2007.12.053

Liberman, I. Y., Shankweiler, D., Fischer, F. W., \& Carter, B. (1974). Explicit syllable and phoneme segmentation in the young child. Journal of Experimental Child Psychology, 18(1074), 201-212. doi:10.1016/0022-0965(74)90101-5

Linkersdörfer, J., Lonnemann, J., Lindberg, S., Hasselhorn, M., \& Fiebach, C. J. (2012). Grey matter alterations co-localize with functional abnormalities in developmental dyslexia: An ALE meta-analysis. PLoS ONE, 7(8). doi:10.1371/journal.pone.0043122

Lyon, G. R., Shaywitz, S. E., \& Shaywitz, B. A. (2003). A definition of dyslexia. Annals of Dyslexia, 53(1), 1-14. doi:10.1007/s11881-003-0001-9

Marino, C., Scifo, P., Della Rosa, P. a., Mascheretti, S., Facoetti, A., Lorusso, M. L., ... Perani, D. (2014). The DCDC2/intron 2 deletion and white matter disorganization: Focus on developmental dyslexia. Cortex, 57, 227-243. doi:10.1016/j.cortex.2014.04.016

McCandliss, B. D., Cohen, L., \& Dehaene, S. (2003). The visual word form area: expertise for reading in the fusiform gyrus. Trends in Cognitive Sciences, 7(7), 293-299. doi:10.1016/S13646613(03)00134-7

McCandliss, B. D., \& Noble, K. G. (2003). The development of reading impairment: a cognitive neuroscience model. Mental Retardation and Developmental Disabilities Research Reviens, 9(3), 196-204. doi:10.1002/mrdd.10080

Mori, S., Crain, B. J., Chacko, V. P., \& Van Zijl, P. C. M. (1999). Three-dimensional tracking of axonal projections in the brain by magnetic resonance imaging. Annals of Neurology, 45(2), 265-269. doi:10.1002/1531-8249(199902)45:2<265::AID-ANA21>3.0.CO;2-3

Nagy, Z., Westerberg, H., \& Klingberg, T. (2004). Maturation of white matter is associated with the development of cognitive functions during childhood. Journal of Cognitive Neuroscience, 16(Figure 1), 1227-1233. doi:10.1162/0898929041920441 


\section{Altered structural connectivity in dyslexia}

Nichols, T., \& Holmes, A. (2002). Nonparametric Permutation Tests for Functional Neuroimaging. In Human Brain Function: Second Edition (Vol. 15, pp. 887-910). doi:10.1016/B978-012264841-0/50048-2

Niogi, S. N., \& McCandliss, B. D. (2006). Left lateralized white matter microstructure accounts for individual differences in reading ability and disability. Neuropsychologia, 44, 2178-2188. doi:10.1016/j.neuropsychologia.2006.01.011

Odegard, T. N., Farris, E. a., Ring, J., McColl, R., \& Black, J. (2009). Brain connectivity in nonreading impaired children and children diagnosed with developmental dyslexia. Neuropsychologia, 47, 1972-1977. doi:10.1016/j.neuropsychologia.2009.03.009

Paulesu, E., Frith, U., Snowling, M., Gallagher, A., Morton, J., Frackowiak, R. S. J., \& Frith, C. D. (1996). Is developmental dyslexia a disconnection syndrome? Brain, 119(1), 143-157. doi:10.1093/brain/119.1.143

Pugh, K. R., Frost, S. J., Sandak, R., Landi, N., Rueckl, J. G., Constable, R. T., ... Mencl, W. E. (2008). Effects of stimulus difficulty and repetition on printed word identification: an fMRI comparison of nonimpaired and reading-disabled adolescent cohorts. Journal of Cognitive Neuroscience, 20(7), 1146-60. doi:10.1162/jocn.2008.20079

Pugh, K. R., Mencl, W. E., Jenner, a R., Katz, L., Frost, S. J., Lee, J. R., ... Shaywitz, B. A. (2001). Neurobiological studies of reaing and reading disability. Journal of Communicative Disorders, 34, 479-492.

Pugh, K. R., Mencl, W. E., Jenner, A. R., Lee, J. R., Katz, L., Frost, S. J., ... Shaywitz, B. A. (2001). Neuroimaging Studies of Reading Development and Reading Disability. Learning Disabilities Research and Practice, 16(4), 240-249. doi:10.1111/0938-8982.00024

Richards, T., Stevenson, J., Crouch, J., Johnson, L. C., Maravilla, K., Stock, P., ... Berninger, V. (2008). Tract-based spatial statistics of diffusion tensor imaging in adults with dyslexia. American Journal of Neuroradiology, 29, 1134-1139. doi:10.3174/ajnr.A1007

Richlan, F., Kronbichler, M., \& Wimmer, H. (2009). Functional abnormalities in the dyslexic brain: A quantitative meta-analysis of neuroimaging studies. Human Brain Mapping, 30(10), 3299-3308. doi:10.1002/hbm.20752

Richlan, F., Kronbichler, M., \& Wimmer, H. (2013). Structural abnormalities in the dyslexic brain: A meta-analysis of voxel-based morphometry studies. Human Brain Mapping, 34(11), 3055-3065. doi:10.1002/hbm.22127

Rimrodt, S. L., Peterson, D. J., Denckla, M. B., Kaufmann, W. E., \& Cutting, L. E. (2010). White matter microstructural differences linked to left perisylvian language network in children with dyslexia. Cortex, 46(6), 739-749. doi:10.1016/j.cortex.2009.07.008

Rohde, G. K., Barnett, a. S., Basser, P. J., Marenco, S., \& Pierpaoli, C. (2004). Comprehensive Approach for Correction of Motion and Distortion in Diffusion-Weighted MRI. Magnetic Resonance in Medicine, 51(1), 103-114. doi:10.1002/mrm.10677 
Sandak, R., Mencl, W. E., Frost, S. J., \& Pugh, K. R. (2004). The Neurobiological Basis of Skilled and Impaired Reading: Recent Findings and New Directions. Scientific Studies of Reading, 8(3), 273-292. doi:10.1207/s1532799xssr0803_6

Saygin, Z. M., Norton, E. S., Osher, D. E., Beach, S. D., Cyr, a. B., Ozernov-Palchik, O., ... Gabrieli, J. D. E. (2013). Tracking the Roots of Reading Ability: White Matter Volume and Integrity Correlate with Phonological Awareness in Prereading and Early-Reading Kindergarten Children. Journal of Neuroscience, 33(33), 13251-13258. doi:10.1523/JNEUROSCI.4383-12.2013

Schlaggar, B. L., \& McCandliss, B. D. (2007). Development of neural systems for reading. Annual Review of Neuroscience, 30, 475-503. doi:10.1146/annurev.neuro.28.061604.135645

Schurz, M., Wimmer, H., Richlan, F., Ludersdorfer, P., Klackl, J., \& Kronbichler, M. (2014). Resting-State and Task-Based Functional Brain Connectivity in Developmental Dyslexia. Cerebral Cortex (New York, N.Y. : 1991), 1-13. doi:10.1093/cercor/bhu184

Share, D. L. (1995). Phonological recoding and self-teaching: sine qua non of reading acquisition. Cognition, 55(2), 151-218; discussion 219-26. Retrieved from http://www.ncbi.nlm.nih.gov/pubmed/7789090

Shaywitz, S. E., Morris, R., \& Shaywitz, B. A. (2008). The education of dyslexic children from childhood to young adulthood. Annual Review of Psychology, 59, 451-75. doi:10.1146/annurev.psych.59.103006.093633

Shaywitz, S. E., \& Shaywitz, B. A. (2005). Dyslexia (specific reading disability). Biological Psychiatry, 57(11), 1301-9. doi:10.1016/j.biopsych.2005.01.043

Simos, P. G., Breier, J. I., Fletcher, J. M., Foorman, B. R., Castillo, E. M., \& Papanicolaou, A. C. (2002). Brain mechanisms for reading words and pseudowords: an integrated approach. Cerebral Cortex (New York, N.Y. : 1991), 12, 297-305. doi:10.1093/cercor/12.3.297

Steinbrink, C., Vogt, K., Kastrup, a., Müller, H. P., Juengling, F. D., Kassubek, J., \& Riecker, a. (2008). The contribution of white and gray matter differences to developmental dyslexia: Insights from DTI and VBM at 3.0 T. Neuropsychologia, 46, 3170-3178. doi:10.1016/j.neuropsychologia.2008.07.015

Thiebaut De Schotten, M., Cohen, L., Amemiya, E., Braga, L. W., \& Dehaene, S. (2014). Learning to read improves the structure of the arcuate fasciculus. Cerebral Cortex, 24, 989995. doi: $10.1093 /$ cercor/bhs383

Turkeltaub, P. E., Gareau, L., Flowers, D. L., Zeffiro, T. a, \& Eden, G. F. (2003). Development of neural mechanisms for reading. Nature Neuroscience, 6(7), 767-73. doi:10.1038/nn1065

Van Atteveldt, N., \& Ansari, D. (2014). How symbols transform brain function: A review in memory of Leo Blomert. Trends in Neuroscience and Education, 1-5.

doi:10.1016/j.tine.2014.04.001 


\section{Altered structural connectivity in dyslexia}

Van der Mark, S., Klaver, P., Bucher, K., Maurer, U., Schulz, E., Brem, S., ... Brandeis, D. (2011). The left occipitotemporal system in reading: Disruption of focal fMRI connectivity to left inferior frontal and inferior parietal language areas in children with dyslexia. NeuroImage, 54(3), 2426-2436. doi:10.1016/j.neuroimage.2010.10.002

Vandermosten, M., Boets, B., Poelmans, H., Sunaert, S., Wouters, J., \& Ghesquière, P. (2012). A tractography study in dyslexia: Neuroanatomic correlates of orthographic, phonological and speech processing. Brain, 135, 935-948. doi:10.1093/brain/awr363

Vandermosten, M., Boets, B., Wouters, J., \& Ghesquière, P. (2012). A qualitative and quantitative review of diffusion tensor imaging studies in reading and dyslexia. Neuroscience and Biobehavioral Reviews, 36(6), 1532-1552. doi:10.1016/j.neubiorev.2012.04.002

Vandermosten, M., Poelmans, H., Sunaert, S., Ghesquière, P., \& Wouters, J. (2013). White matter lateralization and interhemispheric coherence to auditory modulations in normal reading and dyslexic adults. Neuropsychologia, 51(11), 2087-2099. doi:10.1016/j.neuropsychologia.2013.07.008

Vandermosten, M., Vanderauwera, J., Theys, C., De Vos, A., Vanvooren, S., Sunaert, S., ... Ghesquière, P. (2015). A DTI tractography study in pre-readers at risk for dyslexia. Developmental Cognitive Neuroscience, 4-11. doi:10.1016/j.dcn.2015.05.006

Wakana, S., Caprihan, A., Panzenboeck, M. M., Fallon, J. H., Perry, M., Gollub, R. L., ... Mori, S. (2007). Reproducibility of quantitative tractography methods applied to cerebral white matter. NeuroImage, 36(3), 630-644. doi:10.1016/j.neuroimage.2007.02.049

Welcome, S. E., \& Joanisse, M. F. (2014). Individual differences in white matter anatomy predict dissociable components of reading skill in adults. NeuroImage, 96C, 261-275. doi:10.1016/j.neuroimage.2014.03.069

Wimmer, H., \& Schurz, M. (2010). Dyslexia in regular orthographies: manifestation and causation. Dyslexia (Chichester, England), 16(4), 283-99. doi:10.1002/dys.411

Wolf, R. C., Sambataro, F., Lohr, C., Steinbrink, C., Martin, C., \& Vasic, N. (2010). Functional brain network abnormalities during verbal working memory performance in adolescents and young adults with dyslexia. Neuropsychologia, 48, 309-318.

doi:10.1016/j.neuropsychologia.2009.09.020

Yeatman, J. D., Dougherty, R. F., Ben-Shachar, M., \& Wandell, B. a. (2012). Development of white matter and reading skills. Proceedings of the National Academy of Sciences of the United States of America, 109(44), E3045-53. doi:10.1073/pnas.1206792109

Yeatman, J. D., Dougherty, R. F., Myall, N. J., Wandell, B. a., \& Feldman, H. M. (2012). Tract Profiles of White Matter Properties: Automating Fiber-Tract Quantification. PLoS ONE, 7(11). doi:10.1371/journal.pone.0049790

Yeatman, J. D., Dougherty, R. F., Rykhlevskaia, E., Sherbondy, A. J., Deutsch, G. K., Wandell, B. A., \& Ben-Shachar, M. (2011). Anatomical properties of the arcuate fasciculus predict 
phonological and reading skills in children. Journal of Cognitive Neuroscience, 23(11), 3304-17. doi:10.1162/jocn_a_00061

Žarić, G., Fraga González, G., Tijms, J., van der Molen, M. W., Blomert, L., \& Bonte, M. (2014). Reduced neural integration of letters and speech sounds in dyslexic children scales with individual differences in reading fluency. PloS One, 9(10), e110337. doi:10.1371/journal.pone.0110337

Žarić, G., Fraga González, G., Tijms, J., van der Molen, M. W., Blomert, L., \& Bonte, M. (2015). Crossmodal deficit in dyslexic children: practice affects the neural timing of letter-speech sound integration. Frontiers in Human Neuroscience, 369. doi:10.3389/fnhum.2015.00369 



\title{
CHAPTER 6
}

\author{
Summary
}




\section{Summary}

Reading is a complex cognitive function that enables us to rapidly extract meaning from strings of letters (graphemes) that are culturally related to specific speech sounds (phonemes). As writing systems were invented only about 5000 years ago our brains do not have a priori specialized areas for reading. It has thus been proposed that during reading acquisition regions involved in spoken language and vision first adapt to integrate letters and speech sounds and form unified cross-modal representations and second to rapidly recognize familiar words as single units. Though complex, this adaptation is successful in about 9 out of 10 children who acquire reading fluency without major problems. However, some children struggle with reading acquisition and they never fully master this skill. Even when they learn letterspeech sound correspondences and are capable of reading accurately, such as it is often the case in relatively transparent orthographies such as Dutch, they do it slowly and with an effort, i.e. their reading is dysfluent. When these reading problems are persistent and exist despite normal cognitive abilities and educational opportunities, we say that they suffer from developmental dyslexia. Though the body of empirical work is vast the question of underlying neural causes remains open. Do the brains of dyslexics fail to adapt and if yes, at which stage of reading, and at what part of the brain?

The scientific field of cognitive neuroscience offers us a possibility to take a look into the functioning brain by recording its electrical and magnetic properties that are related to the neural and metabolic activity. By using carefully designed experimental paradigms we can assess the neural implementation of specific cognitive functions and compare these in participants belonging to the different groups, for example typical and dyslexic readers and by choosing appropriate statistical methods, we can conclude whether the groups differ in such a way that it is unlikely that the obtained difference is due to the chance. In this thesis we employed multiple neuroimaging methods to assess neural correlates of (dys)fluent reading in 8-10 year old typically reading and dyslexic children. In chapters 2 and 3 we investigated event related potentials, a traditional electrophysiological measure. In chapter 4, we employed a relatively new method of looking into the 
electrophysiological data by evaluating effective connectivity between different channels, allowing us to make inferences about the direction in which information propagates. Finally, in chapter 5, we looked into structural white matter connectivity of the brain employing a diffusion weighted imaging that tells us in what directions the water molecules move in the white matter.

As a growing body of evidence suggests that reading dysfluency is the most persistent and notorious characteristic of dyslexia (Blomert, 2011; Shaywitz, Morris, \& Shaywitz, 2008), this thesis specifically investigates how individual variation in this behavioral measure relates to the functioning of the brain. Thus, we went beyond overall group comparisons and examined more in depth the relation between (dys)fluency and (a)typical brain functioning in dyslexic readers with different levels of reading dysfluency. In chapters 2 and 4, we split our sample of dyslexic children in 2 groups based on their level of reading dysfluency and compared them to each other and to typically reading children. This allowed us to observe differences in electrophysiological measures that scaled with fluency within the dyslexic population, next to more general differences between dyslexic and typical readers.

In chapter 2, we employed a cross-modal oddball paradigm in which a deviant speech sound was violating a standard speech sound (auditory condition) or both a standard speech sound and a visually presented letter (audiovisual condition). The letter was presented either at the same time as the speech sound (synchronous) or it was preceding it for $200 \mathrm{~ms}$ (asynchronous). In each condition, violation due to the deviant speech sound elicited negative ERP responses between 100-250 ms and 600-750ms, corresponding to the mismatch negativity (MMN) and a late negativity (LN). In typically reading children, both negativities were larger in the synchronous and asynchronous crossmodal conditions than in the auditory condition. This crossmodal enhancement of the auditory $\mathrm{MMN}$ and $\mathrm{LN}$ responses indicates early perceptual and later associative integration of letters and speech sounds. Conversely, in both groups of dyslexic children we observed significant letter effects only in the MMN window, and moreover, in severely dysfluent dyslexics only when the letter 


\section{Summary}

preceded the speech sound. Interestingly, the timing of the synchronous crossmodal MMN scaled with dysfluency with a shorter-lasting MMN being related to less fluent reading. This effect was surprising at first but turned out to be due to a lack of a crossmodal deviancy response in the underlying P2 wave in the severely dysfluent dyslexic group. Together these findings suggest that while moderately dysfluent dyslexics have an impaired capability of manipulation of crossmodal representations (as represented by nonsignificant LN letter effects), severely dysfluent dyslexics additionally have a more basic impairment in building clear crossmodal representations (as represented by a nonsignificant MMN letter effect and the lack of a P2 difference in the synchronous condition).

In chapter 3 of this thesis, we investigated whether a training focused on automation of letter-speech sound integration can influence behavioral and electrophysiological (neural responses) measures of this integration in dyslexics. To this end we performed a second EEG measurement in a subgroup of the dyslexic children after they underwent 6 months of an intensive letter-speech sound training in addition to their regular reading curriculum. During the letterspeech sound training they were first explicitly trained in the letter-speech sound correspondences and then exposed intensively to the trained letter speech sound associations to stimulate the automatic integration of letters and speech sounds. Interestingly, when we tested the children with the same crossmodal oddball paradigm as in chapter 2 , we found that the training influenced primarily the timing of the late negativity (LN), with earlier $\mathrm{LN}$ latency at the time of the second measurement (T2) significantly relating to higher behavioral accuracy in letter-speech sound coupling. Due to the size of the sample we could not split dyslexics in the two groups, but correlations of the individual MMN latencies and reading fluency scores, demonstrated a significant relation of the MMN latency to reading fluency at both T1 and T2 as well as with reading gains. This result is an extension of the findings of the chapter 2 , showing not only that the MMN latency related to reading fluency at T1, but was also a predictor of how much training may remediate reading fluency. 
These findings also point towards a more basic impairment in the severely dysfluent dyslexics and suggest that this impairment is hard to remediate.

In chapter 4, we employed a visual word recognition paradigm in the same groups of dyslexic and typically reading children and investigated how effective connectivity in the brain's reading network relates to different levels of reading (dys)fluency. We used directed transfer function (DTF), a method that gives us the opportunity to observe the direction of information propagation between different EEG channels. In this study both dyslexic groups showed weaker connectivity from occipital to inferior-temporal channels than the typical readers. Conversely, dyslexics showed stronger connectivity toward and from central sites indicating a possible involvement of auditory, sensorimotor regions and/or verbal working memory in an attempt to connect orthography to phonology (Blomert, 2011; Pugh et al., 2001; Simos et al., 2002; van Atteveldt \& Ansari, 2014). Interestingly, this connectivity was influenced by the level of reading dysfluency, with moderately dysfluent dyslexics exhibiting stronger connectivity from central to bilateral inferior-temporal sites and the severely dysfluent dyslexic group having stronger connectivity to the right inferiortemporal site. In addition, both groups of dyslexics exhibited stronger connectivity from left frontal to right inferior-temporal sites, possibly suggesting stronger top-down influences during visual word processing as well as the employment of right hemisphere homologues of the typically left dominant reading network. Finally, processing of letter-like meaningless false font symbol strings differentiated mostly between severely dysfluent dyslexic group and the other two groups.

Next to functional connectivity this thesis investigates structural connectivity of the white matter tracts in the reading network of typical and dyslexic readers. Hence, in chapter 5, we employed diffusion weighted imaging, by which we can look at the differences in the structural connections in the brain, through the differences in the diffusion properties of the white matter. Atypical structural properties of white matter bundles within the reading network have been associated with developmental dyslexia, but so far, most studies have compared 


\section{Summary}

dyslexic and typically reading adults with English as their primary language. In chapter 5, we aimed to extend these findings to 9-year old Dutch dyslexic and typically reading children. The same group of children was previously observed to show reduced letter-speech sound integration in a functional magnetic resonance imaging paradigm (Blau et al., 2010). Results of a first analysis of 20 white matter tracts indicated higher fractional anisotropy (FA) of the right thalamic radiation in dyslexic children. Interestingly, across groups higher FA values in this tract were related to more dysfluent reading scores. When we split tracts in hundred equal nodes and calculated FA values at each of these points to investigate tract profiles in more depth we found that the FA values of the posterior part of the bilateral thalamic radiations were related to slower rapid automatized naming. As the thalamic radiations connect subcortical regions with cerebral cortex, and previous studies found increased talamico-cortical connections in dyslexic children toward sensory-motor areas and lateral prefrontal cortex (Fan, Davis, Anderson, \& Cutting, 2014), this increase may represent a prolonged reliance on letter-speech sound connections during reading and/or a compensatory involvement of the working memory. Conversely, typical readers showed a tendency for higher FA values along multiple tracts implicated in the reading, albeit in the right hemisphere, and especially right uncinated fasciculus which also scaled with reading and phoneme deletion accuracy. Our results extend findings on right hemisphere involvement in reading at least in beginning readers. On the other hand, differences in the left hemisphere could be attenuated by the ongoing maturation of the white matter (Yeatman, Dougherty, Ben-Shachar, \& Wandell, 2012), as we found age related changes restricted to the left hemisphere.

As we could see in chapters 2 and 4 the level of dysfluency is an important differentiator within the dyslexic population. Hence, although all dyslexic children show both behavioral impairments and reduced neural letter-speech sound integration and visual word recognition, severely dysfluent group shows indications of more basic impairment (no significant letter effect in both MMN and LN) and less efficient compensatory mechanisms (stronger top down connectivity towards sites on the right side of the head). 
Overall, in this thesis we offer support for the notion that the impairments in neural integration of letters and speech sounds are related to reading dysfluency in children with developmental dyslexia. Moreover, we show that the level of dysfluency has a potential clinical relevance as it was related to all our neuroimaging measures. Furthermore, the training and/or reading instructionrelated gains depended on the severity of letter-speech sound integration impairment with more impaired children being more dysfluent at the beginning and gaining less from the training. Thus, very low scores on reading fluency could represent severe impairments in neural letter-speech sound integration which are more resilient to treatment. Research on the type and intensity of a possible successful treatment in more severe cases is yet to be done. A related question is which neuroimaging measure(s) would be specifically altered by the treatment, next to changes due to the regular reading curriculum or age, and in what way? Finally, the results of this thesis provide support for a letter-speech sound integration deficit as a proximal cause of reading dysfluency in dyslexia (Blomert, 2011). In future studies it would be important to tackle the precise relation of these letter-speech sound integration deficits with the usually observed phonological deficits in individuals with developmental dyslexia.

Instead of a summary, maybe a food for thought: even if we represent brain only as a neural network this is a very complex network not evolved with reading as its goal. Thus, it adapts multiple regions to provide us with this complex capability. There can be multiple reasons why brains cannot successfully adapt. This is yet to be answered. 


\section{Summary}

\section{References}

Blau, V., Reithler, J., van Atteveldt, N., Seitz, J., Gerretsen, P., Goebel, R., \& Blomert, L. (2010). Deviant processing of letters and speech sounds as proximate cause of reading failure: a functional magnetic resonance imaging study of dyslexic children. Brain: A Journal of Neurology, 133(Pt 3), 868-79. doi:10.1093/brain/awp308

Blomert, L. (2011). The neural signature of orthographic-phonological binding in successful and failing reading development. NeuroImage, 57(3), 695-703. doi:10.1016/j.neuroimage.2010.11.003

Fan, Q., Davis, N., Anderson, A. W., \& Cutting, L. E. (2014). Thalamo-cortical connectivity: what can diffusion tractography tell us about reading difficulties in children? Brain Connectivity, 4(6), 428-39. doi:10.1089/brain.2013.0203

Pugh, K. R., Mencl, W. E., Jenner, a R., Katz, L., Frost, S. J., Lee, J. R., ... Shaywitz, B. A. (2001). Neurobiological studies of reaing and reading disability. Journal of Communicative Disorders, 34, 479-492.

Shaywitz, S. E., Morris, R., \& Shaywitz, B. A. (2008). The education of dyslexic children from childhood to young adulthood. Annual Review of Psychology, 59, 451-75. doi:10.1146/annurev.psych.59.103006.093633

Simos, P. G., Breier, J. I., Fletcher, J. M., Foorman, B. R., Castillo, E. M., \& Papanicolaou, A. C. (2002). Brain mechanisms for reading words and pseudowords: an integrated approach. Cerebral Cortex (New York, N.Y. : 1991), 12, 297-305. doi:10.1093/cercor/12.3.297

Van Atteveldt, N., \& Ansari, D. (2014). How symbols transform brain function: A review in memory of Leo Blomert. Trends in Neuroscience and Education, 1-5. doi:10.1016/j.tine.2014.04.001

Yeatman, J. D., Dougherty, R. F., Ben-Shachar, M., \& Wandell, B. a. (2012). Development of white matter and reading skills. Proceedings of the National Academy of Sciences of the United States of America, 109(44), E3045-53. doi:10.1073/pnas.1206792109 
Chapter 7

Knowledge valorization 
A modern society is information based and most of the information is given in a written form. A successful and efficient processing, understanding, and remembering of the information is dependent on an individual's reading skills. Thus, timely and smooth acquisition of reading skills is a sine qua non for the successful completion of education, success in a large percentage of professions and, after all, the easiness of a daily life, foremost in urban environments. As computers and machines take over more and more of manual works from humans, more and more job descriptions are looking for individuals with higher education certificates than just a couple of decades ago. The technological advancements set new requirements for societies, a need for more engineers and programmers, and the increasing population asks for more doctors, dentists, and medical staff in general; more teachers, professors, administrative workers, lawyers, and so on. Although all these professions are very different in most of their demands, they all have one thing in common they require good literacy skills (although anyone who tried to read a doctors' handwriting on a receipt would think differently!). Thus, literacy acquisition is not only important for the wellbeing of an individual, but it is an important skill for the society as a whole. The importance of this twofold social aspect of literacy, for individual members of society and for the society itself, is now recognized and literacy was set as a core goal of the UNESCO's 2006 worldwide campaign to bring "Education to All", with an accent on "the indispensable role that education - with literacy at its core - plays in bettering the lives of individuals, their communities and nations" (UNESCO, 2005).

Although reading is a highly complex and demanding skill, the vast majority of children is capable of mastering it. Nevertheless, one in every ten to twenty children has problems in acquiring proficient reading skills without any accompanying cognitive disability or lack of reading instructions and schooling (Blomert, 2005; Lyon et al., 2003; Snowling, 2013). These children are suffering from developmental dyslexia, "a specific learning disability that is neurobiological in origin" (Lyon et al., 2003). Due to their reduced literacy skills, these children are in danger of adverse academic, economic, and psychosocial consequences (Undheim and Sund, 2008; Undheim et al., 2011). 
Despite substantial advancements in our understanding of the neurobiology of reading and dyslexia, reading dysfluency (a lack of fluency) remains the most persistent and impaired symptom in dyslexic individuals (Shaywitz, Morris, \& Shaywitz, 2008). The NIHC ('Nationaal Initiatief Hersenen en Cognitie') project that funded this $\mathrm{PhD}$ thesis, aimed to gain insight in the brain networks involved in reading fluency development in dyslexia and to improve it through an intervention. As dyslexia is a developmental disorder, we examined neurophysiological correlates of letter-speech sound coupling and visual word recognition in 8-9 year old typical and dyslexic Dutch speaking children. Next to investigating group differences, we specifically focused on differences within the group of dyslexic children based on the severity of reading dysfluency. Moreover the project aimed to examine abnormalities in the reading network, by means of functional and anatomical connectivity at rest and during word reading. Importantly, we crossed the bridge on a gap between science and practice and explored the efficiency a reading training for dyslexic children in terms of both reading improvement and related changes in the brain responses.

As cognitive neuroscience is a relatively young scientific field, our understanding of how the brain processes information is still limited. Especially as we now know that this processing is not constant, but a function of development, i.e. children of different ages may exhibit different brain responses. This thesis, builds upon the existing knowledge on development of neural letter-speech sound integration and word processing by investigating how nine year old children process letters and speech sounds and visually presented words. We focused on neural time course correlates of these processes by measuring EEG in typically reading children and dyslexic children with different levels of reading dysfluency. Moreover, we studied functional and anatomical connectivity within the reading network using EEG measures of visual word recognition and anatomical (DTI) measures of white matter integrity. The children in our study had received 2.5 years of reading instruction ("groep 5" in Dutch educational system). At this stage neural letter-speech sound is still far from adult-like (Froyen et al., 2008, 2009), and, moreover, the 
brain shows increased sensitivity for the orthographic and phonological material compared to both prereading children and adults (Maurer et al., 2006; Bonte and Blomert, 2004; Maurer et al., 2011). Thus, it is important to investigate how a child's brain responds to the basic reading skills, such as letter-speech sound associations, both when the children are successful in mastering reading and when they are not. In this way we are furthering our knowledge of how our brains work, but also we gain deeper understanding of the hurdles encountered by children (and adults) with dyslexia. In this thesis we go a step further and look at the brain responses, not only as a binary category of successful/unsuccessful, but as a continuous function of a reading fluency. This allowed us to investigate individual differences within a group of dyslexic children and to show that the more severe the impairment of reading fluency, the more their brain responses and anatomy differ from those of typically reading children. This type of findings has a twofold importance. First, it can lead towards discovery of neural markers of dyslexia, leading to a better diagnostics. Second, as also indicated in this thesis, it can help in tailoring reading trainings towards the need of specific subgroups, and, ultimately (with the advancement of brain and behavioral measurements and knowledge), of specific individuals.

The goal of this thesis was not only to search for the possible benefits in the future, but to investigate if the currently provided help is efficient and for whom. Thus, we went out of, as some would call it, "a tower of academia" to the society, and related reading gains accompanying a six month reading training with the changes in the brain. Our work shows the benefits of reading practice, both behaviorally and electrophysiologically. First, both latency and amplitude of the late negativity (LN, 600-750ms) could be a marker of a successful letter-speech sound integration, as both dyslexic groups differed in the late negativity window from typical readers, and earlier LN was related to better letter-speech sound coupling after training. Second, it also warrants for the further modification to help the most severely dysfluent readers, as the timing of the early crossmodal change detection responses (MMN, 100-250ms), that significantly differed between the groups based on fluency, may provide a 
biomarker that could contribute to a better prediction of reading gains and/or individual tailoring of dyslexia training/intervention strategies (Leppänen, 2013).

Consequently, the results presented in this thesis are of interest to individuals suffering from dyslexia and their families, especially the parents of the children with developmental dyslexia, professionals providing reading trainings, such as Regionaal Instituut Dyslexie (RID) and IWAL Instituut voor leerproblemen in the Netherlands, for educational institutions, to adapt exams, e.g. longer time for written exams, for the pupils/students with dyslexia, and finally to provide lawmakers with specific and objective criteria with respect to diagnostics and reading trainings to guide their policy with respect to healthcare insurance, e.g. as is currently the case in the Netherlands.

\section{References}

Blomert, L. (2005). Dyslexie in Nederland. Amsterdam: Uitgeverij Nieuwezijds Available at: http://www.boomtestuitgevers.nl/upload/Dyslexie_in_Nederland_Leo_Blomert.pdf [Accessed April 16, 2014].

Bonte, M. L., and Blomert, L. (2004). Developmental dyslexia: ERP correlates of anomalous phonological processing during spoken word recognition. Brain Res. Cogn. Brain Res. 21, 360-76. doi:10.1016/j.cogbrainres.2004.06.010.

Froyen, D., Van Atteveldt, N., Bonte, M., and Blomert, L. (2008). Cross-modal enhancement of the MMN to speech-sounds indicates early and automatic integration of letters and speech-sounds. Neurosci. Lett. 430, 23-8. doi:10.1016/j.neulet.2007.10.014.

Froyen, D., Bonte, M. L., van Atteveldt, N., and Blomert, L. (2009). The long road to automation: neurocognitive development of letter-speech sound processing. J. Cogn. Neurosci. 21, 567-80. doi:10.1162/jocn.2009.21061.

Leppänen, P. H. T. (2013). Introduction to the special issue on brain event-related potentials as biomarkers of language and literacy development, feedback, and intervention. Dev. Neuropsychol. 38, 507-13. doi:10.1080/87565641.2013.829842.

Lyon, G. R., Shaywitz, S. E., and Shaywitz, B. A. (2003). A definition of dyslexia. Ann. Dyslexia 53, 1-14. doi:10.1007/s11881-003-0001-9.

Maurer, U., Brem, S., Kranz, F., Bucher, K., Benz, R., Halder, P., Steinhausen, H.-C., and Brandeis, D. (2006). Coarse neural tuning for print peaks when children learn to read. Neuroimage 33, 749-58. doi:10.1016/j.neuroimage.2006.06.025.

Maurer, U., Schulz, E., Brem, S., der Mark, S. Van, Bucher, K., Martin, E., and Brandeis, D. (2011). The development of print tuning in children with dyslexia: evidence from longitudinal ERP data supported by fMRI. Neuroimage 57, 714-22. doi:10.1016/j.neuroimage.2010.10.055.

Shaywitz, S. E., Morris, R., and Shaywitz, B. A. (2008). The education of dyslexic children from childhood to young adulthood. Annu. Rev. Psychol. 59, 451-75.

doi:10.1146/annurev.psych.59.103006.093633. 


\section{Knowledge valorization}

Snowling, M. J. (2013). Early identification and interventions for dyslexia: a contemporary view. J. Res. Spec. Educ. Needs 13, 7-14. doi:10.1111/j.1471-3802.2012.01262.x.

Undheim, A. M., and Sund, A. M. (2008). Psychosocial factors and reading difficulties: Students with reading difficulties drawn from a representative population sample. Scand. J. Psychol. 49, 377-384. doi:10.1111/j.1467-9450.2008.00661.x.

Undheim, A. M., Wichstrøm, L., and Sund, A. M. (2011). Emotional and Behavioral Problems Among School Adolescents With and Without Reading Difficulties as Measured by the Youth Self-Report: A One-Year Follow-Up Study. Scand. J. Educ. Res. 55, 291-305. doi:10.1080/00313831.2011.576879.

UNESCO (2005). Education for all: Literacy for life. 
Acknowledgements 


\section{Acknowledgements}

When I moved to Maastricht, back in August of 2009, I thought it would be only for a period of my master studies. Fortunately, I was wrong. Now, six and a half years later, I find myself finishing a PhD that connected me to many people without whom my professional, and more importantly, personal experience would be much poorer.

I would first like to thank my scientific "mother" "big sister", Milene Bonte, for being, in a word, Milene. This project would definitely not be of the same quality and finished on time if you did not devote yourself to it. Milene, the doors of your office were always open for me when I had a doubt. You were able to stir my wild interpretations into scientific thoughts, and taught me to smile when I talk about my work. You were always sincere and direct, that made my $\mathrm{PhD}$ a very pleasant journey. You are my supervisor, but I am happy to say, you are also a friend.

Next, I would like to thank Leo, for believing in me and offering me the task to finish his last project. Unfortunately, I knew Leo only for a short time, but in this period he struck me as a unique character, and someone who I would like to resemble in his passion for life and work and his uncompromisable attitude towards bullsh*t in this world. This project turned out to be Leo's swan song, and though he was definitely not a silent swan during his life, I hope that he would approve and be proud of this work.

¡Gorka! Gorkito! Amigo! Cuántos momentos! Oh, what a time we had! You are a great fellow worker, fellow partyer and fellow fellow! I don't know where to start, from your hospitability in Amsterdam, your endurance against my puns, our almost daily chats (both work and life related) or the great time we had at your parents' place in Getxo. For me, Amsterdam will always be inseparable from you, the extra mattress on the floor of your room at Montelbaanstraat 4, evening siestas before going out, flamenco, café Cuba at Nieuwmarkt and the smell of the warm ham and cheese croissants from Albert Heijn in Jodenbreestraat that we would pick up in the morning on our way to collect data. We managed to collect our data even though the circumstances were not always on our side! Like the movement of the UvA psychology department and its EEG labs, or the last minute modifications of the experimental sessions asked by the ethical committee. Or even the broken heating in your house in February 2012 with outside temperature falling to minus $18^{\circ} \mathrm{C}$ and inside temperature not lagging far behind! Remember how we looked so frozen and miserable that a waitress from the café Cuba offered us to stay at her place (Thank you Merche for saving us from freezing!). 
I want to thank Maurits and Jurgen, my co-supervisors from the Universiteit van Amsterdam. Maurits thank you for taking over an organizational part from Leo without which this project wouldn't have been possible, as well as for the fruitful discussions of the results and careful reading and suggestions to the manuscripts. Jurgen, thank you for all your hard work, for all the behavioral testing, participants recruitment and group randomization, and for your friendliness and helpfulness. Thank you for immediately accepting to give a talk at EPOS $\mathrm{PhD}$ day and for sending me those two 'voetbalplaatjes' that I was missing in the "EURO 2012 AH Family editie" album.

A special thanks goes to Bernadette, for taking over as my promotor when needed and keeping sure that everything is finished on time, and for the cozy group dinners at her place. And while we are at Bernadette's place, thanks to Hank for letting us into his man-cave and performing private concerts for us. For those about to rock, we salute you!

Ai, João, 'carapau'! It took me some time to realize that you actually do not live in café Zondag. Sometimes we are like those two goats on the bridge, some would even say like an "old couple" (and by "some" I mean everybody except the two of us), but most of the time we are having lots of fun (and) making a lot of fun of each other and our southern macho personas. Thank you for keeping up with my low threshold for getting annoyed when I am under stress. Thank you for friendship, dinners, beers and beer counting, gym, DTF, "Mandala" (that amazing restaurant in Rhodes, that we ended up dining in every evening), road-trip around lake Michigan and that face that you make when Barcelona wins with 4 goals over Real. And thank you for having such a nice girlfriend (seriously, Carolin, how do you keep up with him!), so that I can talk to someone normal when we go out.

"F" is for Phonetic-man (and Feuerzangenbowle)! Dr. Lars Housemate, thank you for all your calmness and joyfulness that you spread around. I'll always cherish all those indispensable evenings in your kitchen in front of the first piece of "art" (quotation marks debatable) that you acquired, when we discussed everything from "dog" to "god" with conversations becoming more and more surreal as time flew by while significant amounts of beer or wine and sometimes even tea (sic!) disappeared; our summer holiday in Montenegro and weekend getaways in Ardennes; your "dancing" moves and humming to the food. And I'll always remember how you tried to make me die of heart attack over and over again by inviting me to join you on your "short" $10 \mathrm{~km}+$ runs that always involved some path leading uphill. 


\section{Acknowledgements}

Just a wall away (at least since the department moved in to the new building) are my two comrades from masters. Mario, thanks for all the liters of coffee that we drank while making increasingly complex and absurd jokes and the shared interest in finding and/or preparing the best burger in the world. May we have many more BBQs (and Anna's gyros)! Mehrdad, if you become a cook you would not make a wrong carrier choice. You made me totally hooked to saffron rice and lamb stews. But let's pretend that you never brought "rocky mountain oysters" to my birthday BBQ. And one more thing, I am not going to forget that last hand full-house (fives full of queens) from last December. Rematch it is! I know that Sanne and you tried to make me join you in improve theatre, and I don't mind if you continue trying. And Sanne, thanks for all the talks, drinks and that deck of cards that, to my horror, I can't erase from my memory. Joining them in the office was Arne, always ready for jokes and talks on mathematics; hope you get some sleep, "vati”.

During my PhD I shared the office with some great people. Anniek Vaessen helped me at the beginning when, frankly, I had no idea what I was supposed to be doing. Katie Wheat, who was a great companion for morning coffee chats and Friday afternoon drinks and everything in between. Anna Zilverstand, whose great drawing made our office more homely and personal. Faruk, who programs in all known and unknown free-license programs. He helped me to find my way in the vastness of a Debian virtual machine and mused with me over parameters for DTI analysis. And last but not least, Lukas, thanks for showing João and me that we are more camping in the gym than working out, and for all the peanuts. It would be unfair not to mention people that are for sure my officemates in some other dimension, but due to some quantum uncertainties show up in this one very often. Yes, Tahnée and Helen, I am talking about you. Heisenberg's uncertainty principle states that although I know that it is Helen speaking, I have no idea what she is saying. But that could just be because she usually speaks German to Lukas. On the other hand, Schrödinger's Tahnée is and is not in the office at the same time, preferring to stand in the door frame.

Kamil, you were a great head of the department and you are a great friend. I know that we'll continue our fruitless effort of explaining to João that it cannot be a penalty for Real if Ronaldo self-fouls himself out of the penalty area, and especially if he does so against Barcelona. May we share many more pop-corns in Euroscoop! Aline, you are probably reading this in London, because I assume that you can't have anything more interesting to do there! We'll go drinking again (no bikes for sure!), just be patient till I don't need visa to visit you! Martin, or better, 'MarcIn, to "pronounce" it correctly, at 
least on a paper, you'll maybe read this, but I know who'll for sure laugh at my jokes, as Thessely always does. It is rumored that soon we should have squash at UM sports, so I'll be able to pursue my beloved activity of losing miserably against you. Matteo, you are already in Oxford, and even if this does not reach you, thanks for helping with DTI preprocessing, and those great spaghetti and tiramisu and much more. I always laugh when I watch your improvisation acting in João's movie. There is definitely an unpursued talent there. Keep on rocking, John Bon Jovi! Inge, thank you for all your help with the DTI analysis and explaining it all from scratch to me. Remember how we discovered together how wrongly oriented tensors look?!

Not to forget my other housemates! Anna, our Italian "mammina” preparing all the delicious Italian dishes and to my surprise usually stating: "It only takes $<30$ minutes to make ___ (insert any food that you prepare)". I always feel amazed when I see your will to go out in a misty and rainy Belgian night to hit the ball over the net in a first league. Shanice, who finds my pronunciation of Dutch words very funny. Soon we will be able to go out again, exams are almost over! And my short term housemate Britta whose (unfortunately rare) visits are always causes for a lot of laughs and joy. And finally, the guy whose apartment I was lucky enough to take over, Alexandros Goulas. The man who boldly went where no man had gone before (backed up by Lars) and refused to describe his beer taste to a certain café owner. Thus, we don't go to "Take One" anymore.

Christl, Riny, Eva and Annemie, thank you for bearing with me, my forms and questions. I know that it was not an easy task: from an instrument arriving from the USA, and all the NS business cards that I came to pick up at the last moment, and then forgot to return on a specified day, to all the forms that you had to take care of. Here, I would also like to express gratitude to Martine Berghof for being extremely helpful and friendly.

Thank you, Amanda and Joel, for allowing me to make my first task in a course manual for students. And thanks to Alard with whom I made my first steps of fMRI data preprocessing and constructing beautiful 3D meshes as his student assistant.

The first year of my $\mathrm{PhD}$ that included a lot of traveling between Amsterdam and Maastricht, and very little time in the office to meet new people would not be as good without some familiar faces around, like David Niermann, Robert Panne and Alex 


\section{Acknowledgements}

Dusik. And, of course, annual visits by Diana Müssgens on her trip home from Sweden.

Although not strictly part of my $\mathrm{PhD}$, but present during it as we tried to make my master thesis publishable (and we finally did it!), Peter De Weerd, Arash Yazdanbakhsh Shigeaki Nishina and Takeo Watanabe, thank you for all the patience and help.

Our department grew a lot in the meantime so I am bound to forget someone who made me smile, but don't worry, the connection between you and smiling is recorded in my brain: Marin, Rosanne, Jan, Valentin, Arko, Mario, Sanae, Gesa, Felix, Shri, Teresa, Alex, Niels, Julia, Vittoria, Roberta, Thomas, Franziska, Francesco, Martin, Elia, Giancarlo, Federico, Jeannette, Dennis, Kirsten, Kiki, Judith, Rainer, Joost, Michelle, Job, Tom, Arie, Michael, Bert, Martha, Jessica, Bettina, Lars, Fren, Ingo, Marian, Shruti, Tabea,... and some great people outside of the department, Christine Gutekunst, Julie, Jo, Ehsan, Ali,... and some beautiful people I met in Ghent, that enriched my life: Hsing-Yi and Romain, Selene and Jonathan, Stephany and Tim, Igor, Naomi, Ine, Valentina, Inez and Antonio, Maud and Minh,...

I would like to thank my friends and family from Serbia, still there or scattered around Europe, who haven't forgot me and that still make our reunions look like the time has stopped and we have never parted: Matija Obradović, Vladimir Kralj, Marko Cvetković, Dubravka Subotić, Ana Anđelić, Tamara Dimitrijević, Igor Stanojević, Slobodan Popović, Milutin Trnavac, Danko Mišić, Nevena Đoković, Marko Kalanj, Stepa Savatić,...

Jasmina, my love, you had to put up with me finishing this, and you moved to a different city/country so that we can be together in our cozy "box of matches". Thank you for everything.

Finally, Vukica, my mother, I dedicate this thesis to you, for everything you are and you do / Мама, теби посвећујем ову тезу, због свега што јеси и што радиш. 


\section{Publications}

Žarić, G., Fraga González, G., Tijms, J., van der Molen, M. W., Blomert, L., \& Bonte, M. (2015). Crossmodal deficit in dyslexic children: practice affects the neural timing of letter-speech sound integration. Frontiers in Human Neuroscience, 9:369.

Fraga González, G., Žarić, G., Tijms, J., Bonte, M., Blomert, L., \& van der Molen, M.W. (2015). A randomized controlled trial on the beneficial effects of training letter-speech sound integration on reading fluency in children with dyslexia. PLOS One, 10(12), e0143914.

Žarić, G., Fraga González, G., Tijms, J., van der Molen, M. W., Blomert, L., \& Bonte, M. (2014). Reduced neural integration of letters and speech sounds in dyslexic children scales with individual differences in reading fluency. PLOS One, 9(10), e110337.

Fraga González, G., Žarić, G., Tijms, J., Bonte, M., Blomert, L., \& van der Molen, M. W. (2014). Brain-potential analysis of visual word recognition in dyslexics and typically reading children. Frontiers in Human Neuroscience, 8:474.

Žarić, G., Yazdanbakhsh, A., Nishina, S., De Weerd, P., \& Watanabe, T. (2015). Perceived temporal asynchrony between sinusoidally modulated luminance and depth. Journal of Vision, 15(15):13. 
